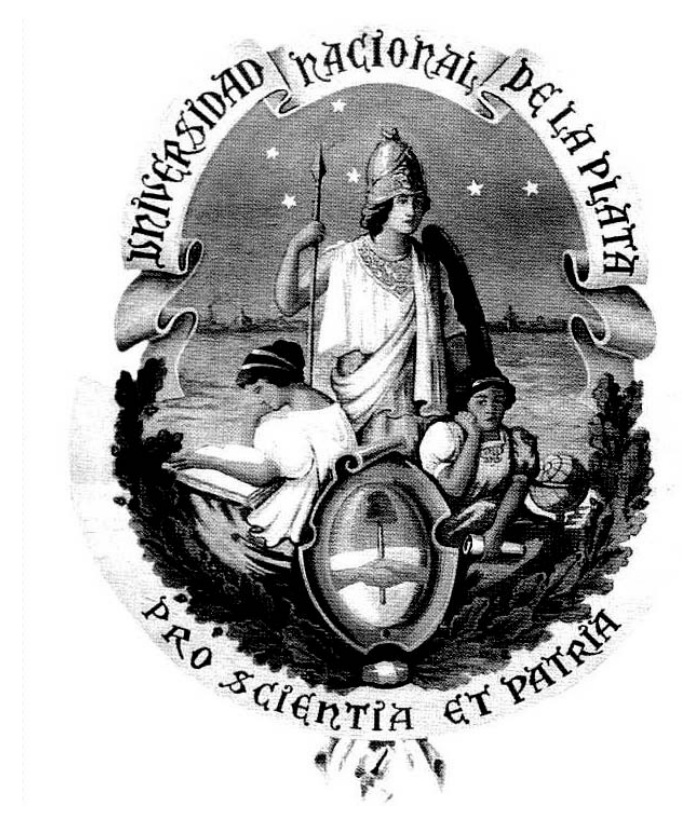

Universidad Nacional de La Plata

Facultad de Ciencias Astronómicas y Geofísicas

Tesis para obtener el grado académico de Doctora en Astronomía

\title{
COMPARACIÓN DE LAS PREDICCIONES DE COSMOLOGÍAS ALTERNATIVAS AL MODELO COSMOLÓGICO ESTÁNDAR CON DATOS DEL FONDO Cósmico DE RADiación
}

María Pía Piccirilli

Directora: Dra. Susana Landau

Codirectora: Dra. Claudia Scóccola 



\title{
Prefacio
}

Esta Tesis es presentada como parte de los requisitos para obtener el grado académico de Doctora en Astronomía de la Universidad Nacional de La Plata. La misma contiene los resultados de los estudios desarrollados bajo la dirección de la Dra. Susana Landau y la codirección de la Dra. Claudia Scóccola, y en colaboración con otros investigadores de la Universidad de Buenos Aires, la Universidad Nacional Autónoma de México y el Observatorio Nacional de Río de Janeiro; entre los años 2012 y 2018.

\author{
María Pía Piccirilli. \\ e-mail: mpp@fcaglp.unlp.edu.ar \\ La Plata, mayo de 2018.
}

Fecha de defensa: jueves 28 de junio de 2018 . Fecha de la copia actual: 11 de febrero de 2019. 



\section{Resumen}

El modelo cosmológico estándar postula el origen del Universo como un evento singular y un suceso de eventos característicos a los que comúnmente se refiere como Big Bang. En el marco del modelo cosmológico estándar surgen algunas dificultades ampliamente discutidas en la literatura como el problema de los horizontes, la planalidad, la formación de estructuras, entre otros. La solución propuesta en este marco es contemplar una etapa temprana del Universo de expansión acelerada que se denomina inflación.

Sin embargo, no se encuentra bien fundamentado el surgimiento de un Universo anisótropo e inhomogéneo a partir de un estado inicial completamente isótropo y homogéneo. Esta transición no puede ser explicada por la evolución unitaria usual de la mecánica cuántica, debido a que dicha evolución preserva las simetrías iniciales. Para solucionar este problema, el Dr. Daniel Sudarsky y colaboradores han propuesto introducir el colapso autoinducido de la función de onda del campo inflatón como el mecanismo responsable de la ruptura de simetrías. Para describir la dinámica del colapso se llevan a cabo distintos enfoques: i) esquemas de colapso, donde en un determinado tiempo de colapso la función de onda del campo inflatón y/o su campo conjugado modifican sus valores de expectación, dando lugar a un estado post-colapso que ya no comparte las simetrías del estado vacío previo al colapso; ii) método de Localización Continua y Espontánea (CSL), donde a través de la modificación de las ecuaciones de la mecánica cuántica que rigen la dinámica del campo inflatón, se obtiene un estado post-colapso como parte de la evolución natural del sistema. Dentro de la primera propuesta se desarrollan modelos teóricos en los cuales el colapso suceda durante el período inflacionario así como también durante la época de radiación, obteniendo distintos resultados para ambas propuestas.

Los objetivos de esta tesis son: desarrollar los modelos teóricos descriptos anteriormente y obtener sus predicciones tal que puedan ser comparadas con los datos, y realizar los análisis estadísticos que puedan determinar la validez de los mismos y restringir sus parámetros libres. Se exponen los resultados de variados análisis estadísticos cuyo objetivo principal es comparar las predicciones teóricas de los modelos alternativos al modelo cosmológico estándar con datos del Fondo Cósmico de Radiación y cartografiado de Galaxias, entre otros. Se estiman los parámetros libres para cada caso, previo estudio de la viabilidad de cada propuesta. Algunos esquemas resultan favorecidos por los datos y los indicadores estadísticos frente al modelo cosmológico estándar. La importancia de este resultado es poder contar con un modelo teórico que sea capaz de explicar claramente el origen de las inhomogeneidades y anisotropías del Universo temprano que tienen como consecuencia el surgimiento posterior de la estructura a gran escala. 



\section{Agradecimientos}

Al equipo de Supercómputo de la Universidad Nacional Autónoma de México por el acceso a una valiosísima herramienta de trabajo como lo es la supercomputadora Miztli, sin la cual esta tesis no se habría podido llevar a cabo. 

Esta tesis es el resultado de muchas contribuciones y, sobre todo, mucho esfuerzo.

En primera medida, es fruto de un gran equipo de trabajo muy capaz, colaborador, presente y, por sobre todas las cosas, solidario.

También es el resultado de un apoyo familiar incondicional. Sin la confianza ciega de la familia y aquellos amigos-familia, no hay doctorado posible.

Pero por sobre todas las cosas, esta tesis es una construcción.

Una construcción donde la ciencia, la tecnología y la educación pública, gratuita y de calidad fueron un pilar fundamental.

Gracias a todos los que construyeron junto a mí. 



\section{Índice general}

$\begin{array}{lll}\text { Prefacio } & \text { III }\end{array}$

Resumen $\quad$ V

1. Observables Cosmológicos 1

1.1. Introducción . . . . . . . . . . . . . . . . . . . . . . 1

1.2. Breve historia del Fondo Cósmico de Radiación . . . . . . . . . . . . . . . . 1

1.3. Observaciones del Fondo Cósmico de Radiación . . . . . . . . . . . . . . . 2

1.4. Formación de átomos neutros en el Universo temprano . . . . . . . . . . . . . 4

1.5. La física de la recombinación $\ldots \ldots \ldots \ldots \ldots$

1.6. Observables del Fondo Cósmico de Radiación . . . . . . . . . . . . . . . 6

1.7. Espectro de temperatura y polarización del FCR . . . . . . . . . . . 8

1.8. Oscilaciones Acústicas de Bariones . . . . . . . . . . . . . . . . . . 10

1.8.1. Observables de las Oscilaciones Acústicas de Bariones . . . . . . . . . 12

1.8.2. Datos utilizados para BAO . . . . . . . . . . . . . . 13

1.9. Telescopio Espacial Hubble . . . . . . . . . . . . . . . . . . . . . . . 13

2. Análisis Estadístico 15

2.1. Introducción . . . . . . . . . . . . . . . . . . . . 15

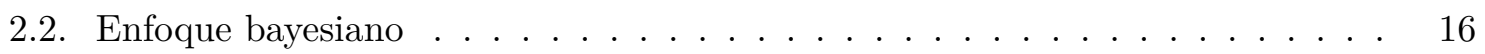

2.3. Cadenas de Markov-Monte Carlo . . . . . . . . . . . . . . . . . . . 16

2.3.1. Integración Monte Carlo . . . . . . . . . . . . . . . . 17

2.3.2. Cadenas de Markov . . . . . . . . . . . . . . . . 17

2.3.3. Algoritmo de Metropolis-Hastings . . . . . . . . . . . . . . . 17

2.3.4. Convergencia . . . . . . . . . . . . . . . . . 18

2.4. Función de Likelihood para el FCR . . . . . . . . . . . . . . . . . 20

2.4.1. Evidencia Bayesiana . . . . . . . . . . . . . . . . . 20

2.5. Códigos numéricos . . . . . . . . . . . . . . . . . . 21 
3. Inflación $\quad 23$

3.1. Introducción . . . . . . . . . . . . . . . . . . . . . . . . 23

3.2. El problema del horizonte . . . . . . . . . . . . . . . . . . . . . 24

3.3. Problema de la planalidad . . . . . . . . . . . . . . . . . . . . 27

3.4. Expansión acelerada del Universo como solución al problema de los horizontes 29

3.5. Duración de inflación . . . . . . . . . . . . . . . . . . . . . 29

3.6. La física del período inflacionario . . . . . . . . . . . . . . . . . 31

3.7. Inflación slow-roll . . . . . . . . . . . . . . . . . . . . . . . . . . . 32

3.8. El problema de la ruptura de simetrías . . . . . . . . . . . . . . . . . . 34

4. Modelos de colapso 35

4.1. Introducción . . . . . . . . . . . . . . . . . . . . . . . . 35

4.2. La aproximación semiclásica . . . . . . . . . . . . . . . . . 36

4.3. Antecedentes . . . . . . . . . . . . . . . . . . . . . 38

4.4. Modelos cuasi-de Sitter . . . . . . . . . . . . . . . . . . . . . . 38

4.4.1. Cuantización y caracterización del colapso . . . . . . . . . . . . . . . . 40

4.4.2. Esquemas de colapso . . . . . . . . . . . . . . . . . . . 43

4.4.3. Perturbaciones en la curvatura . . . . . . . . . . . . . . . . 45

4.4.4. Cálculo del espectro primordial de fluctuaciones . . . . . . . . . . . . . 48

4.4.5. Comparación entre los espectros de potencias . . . . . . . . . . . . . 52

4.4.6. Esquemas dentro y fuera del radio de Hubble . . . . . . . . . . . . . 53

4.4.7. Comparación del espectro primordial de los modelos de colapso con el del modelo cosmológico estándar . . . . . . . . . . . . . . . . . 55

4.4.8. Espectro angular de anisotropías del Fondo Cósmico de Radiación . . 57

4.5. Colapso mediante el mecanismo CSL . . . . . . . . . . . . . . . . . . . . . 61

4.5.1. Espectro primordial de potencias . . . . . . . . . . . . . 64

4.5.2. Espectro de Anisotropías . . . . . . . . . . . . . . 65

4.6. Colapso durante el período de radiación . . . . . . . . . . . . . . 66

4.6.1. Análisis de las perturbaciones cuánticas . . . . . . . . . . . . . . . . . 69

4.6.2. Perturbaciones en la curvatura . . . . . . . . . . . . . . 70

4.6.3. Cantidades observables . . . . . . . . . . . . . . . . . . 71

4.6.4. Análisis del espectro de potencias . . . . . . . . . . . . . . . 72

4.6.5. Espectros de potencias y anisotropías angulares . . . . . . . . . 76

4.7. Selección de Modelos Teóricos para el análisis estadístico . . . . . . . . . . . . 78 
$\begin{array}{lr}\text { 5. Resultados } & 81\end{array}$

5.1. Esquemas de colapso . . . . . . . . . . . . . . . . . . . 83

5.1.1. Análisis estadísticos con datos de Planck2015 . . . . . . . . . . . 83

5.1.2. Análisis estadísticos con datos de Planck2015 + BAO . . . . . . . . 88

5.1.3. Análisis estadísticos con datos de Planck2015+BAO+HST . . . . . . . 92

5.2. Modelo CSL . . . . . . . . . . . . . . . . . . . . . . . 93

5.3. Potenciales de inflación . . . . . . . . . . . . . . . . 97

$\begin{array}{ll}\text { 6. Conclusiones } & 101\end{array}$

$\begin{array}{ll}\text { A. Acrónimos } & 103\end{array}$ 



\section{Capítulo 1}

\section{Observables Cosmológicos}

\subsection{Introducción}

El estudio del Fondo Cósmico de Radiación (FCR) es una de las herramientas más importantes con las que se cuenta en la actualidad para el estudio de la física del Universo temprano.

El Universo primordial estaba formado por un plasma donde protones y electrones se encontraban acoplados con la radiación debido a la interacción constante entre fotones y electrones libres mediante el scattering de Thomson. Alrededor de 380.000 años luego de la singularidad inicial, la temperatura del Universo descendió por debajo de la energía correspondiente a la ionización del átomo de hidrógeno debido a la expansión del Universo. Protones y electrones se combinaron para formar átomos de hidrógeno neutro, disminuyendo la densidad numérica de electrones libres y, por ende, su interacción con los fotones. A este proceso se lo denomina técnicamente recombinación.

A medida que electrones y protones se combinaron para formar átomos de hidrógeno neutro, disminuyó progresivamente la densidad de electrones libres disponibles para interactuar mediante scattering con los fotones, dando lugar a la etapa que se denomina desacople. Previo a esto, el Universo era opaco a la radiación. Luego del desacople, los fotones se ven libres de viajar a través del espacio, llevando consigo información acerca de la física de las primeras etapas del Universo y conformando lo que actualmente se denomina el FCR.

Las secciones de este capítulo están basadas en el libro de S. Weinberg "Los tres primeros minutos del Universo", en el libro de B. Ryden "Introduction to Cosmology" y en el artículo "Cosmological constraints from baryonic acoustic oscillation measurements" de scholarpedia.org.

\subsection{Breve historia del Fondo Cósmico de Radiación}

El 1915 la teoría de la Relatividad General de Einstein sentó las bases para poder describir el Universo y predecir su evolución. Georges Lemaître resolvió las ecuaciones de Einstein que describen un Universo en expansión, que luego fue confirmado observacionalmente por Hubble en 1929 con el corrimiento al rojo de galaxias lejanas (redshift de ahora en más). Si el Universo se expandió desde su origen, las características en las que se presenta la materia fueron cambiando junto a su evolución. Aproximadamente 380.000 años luego de la singularidad 
inicial, el Universo primigenio consistió en un plasma de electrones, protones y fotones en interacción constante.

Hacia fines de los años 1940 George Gamow, Ralph Alpher y Robert Herman realizaron cálculos acerca de las condiciones físicas del plasma de materia que conformaba el Universo temprano y predijeron la existencia de una radiación remanente cuyo espectro correspondía al de un cuerpo negro a 5 K. Más adelante, en los años 1960, Yakov Zel'dovich en la Unión Soviética, y Fred Hoyle y R. J. Tayler en Gran Bretaña redescubrieron de manera independiente esta predicción y mejoraron las estimaciones. Robert Dicke hacia 1964 había comenzado a preguntarse sobre la posibilidad de observar dicha radiación. Inmediatamente P. G. Roll y D. T. Wilkinson comenzaron la búsqueda de la misma.

Paralelamente, P. J. E. Peebles estimó que de mantenerse la producción de helio y elementos más pesados en los primeros minutos dentro de los límites conocidos, la radiación de fondo tuvo que haber sido tan intensa de modo que su temperatura actual sea de al menos $10 \mathrm{~K}$ (cálculo que luego fue perfeccionado). Finalmente, la radiación fue detectada por Arno Penzias y Robert Woodrow Wilson como un exceso en la temperatura de antena con la que buscaban medir señales de radio provenientes de un satélite. Las características particulares de este "ruido de radio" (isotropía, independencia estacional, subsistencia ante distintas pruebas de eliminación de ruido, etc.) dio lugar a que Peebles y sus colegas identificaran como la radiación buscada. Esta es la primera detección observacional del FCR.

Durante los últimos 25 años se desarrollaron una amplia variedad de experimentos para medir el FCR, tanto en telescopios de base terrestre como en globos atmosféricos, entre ellos BOOMERanG, MAXIMA, DASI, QUaD, Atacama Cosmology Telescope (ACT), South Pole Telescope (SPT), QUIET, etc. Hacia principios de los años 1990, la misión espacial Cosmic Background Explorer (COBE) de la NASA confirmó con precisión la isotropía de la radiación y el espectro de cuerpo negro con temperatura de $2.725 \mathrm{~K}$, construyendo también el primer relevamiento del cielo completo. Luego las colaboraciones de los satélites Wilkinson Microwave Anisotropy Prove (WMAP-NASA) lanzado en 2001 y Planck (ESA) lanzado en 2009, revelaron las anisotropías del orden de $10^{-5}$ que son huella de la física del Universo temprano.

Como toda radiación electromagnética, los fotones no sólo proveen información acerca de la temperatura del Universo temprano a través de la medición de su intensidad, si no que también proveen datos complementarios a través del estudio de su polarización.

\subsection{Observaciones del Fondo Cósmico de Radiación}

El espectro del Fondo Cósmico de Radiación (FCR) puede identificarse con el de un cuerpo negro con gran precisión, como muestra la figura 1.1. Su distribución espectral de energía corresponde a una temperatura promedio $T_{0}=2.725 \mathrm{~K}$ en todas las direcciones del cielo, hasta un orden de $10^{-3}$. Aumentando la precisión para la medición en la temperatura un orden de magnitud, se observa un patrón conocido como "dipolo", donde la temperatura en una mitad del cielo está más alta que en la mitad opuesta. Esto no forma parte de la física intrínseca de la radiación si no que es una distorsión local debida al movimiento de la Tierra respecto a un marco de referencia en el cual el FCR es isótropo.

Este efecto puede restarse para estudiar las contribuciones netas de las anisotropías cuya amplitud es muy pequeña. Si la temperatura en cierta dirección es $T(\theta, \phi)$, el promedio en 


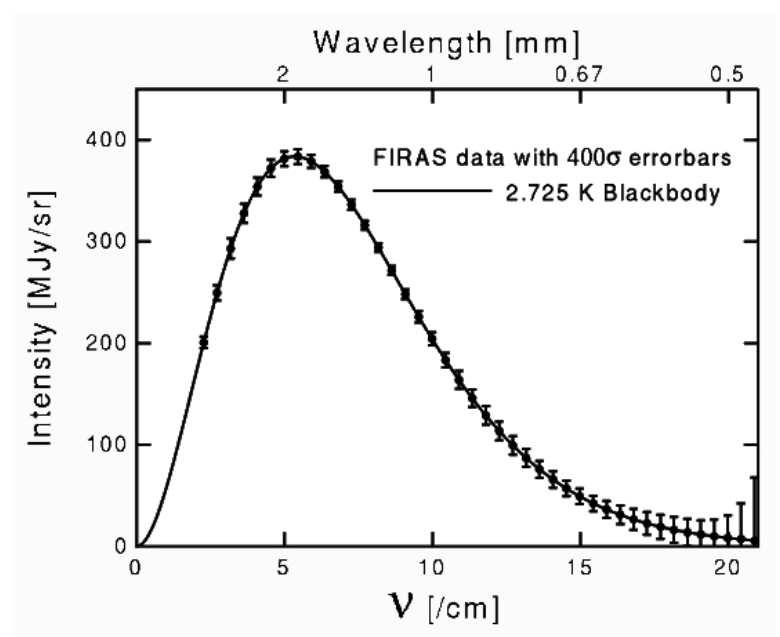

Figura 1.1. Espectro de potencias para la medición del FCR, que se corresponde con gran precisión al de un cuerpo negro a $2.725 \mathrm{~K}$. Las anisotropías se hacen visibles recién al quinto decimal en la temperatura. Créditos: NASA JPL, Caltech.

todo el cielo resulta

$$
\langle T\rangle=T_{0}=\frac{1}{4 \pi} \int T(\theta, \phi) \sin (\theta) d \theta d \phi=2.725 \mathrm{~K} .
$$

Las fluctuaciones de temperatura relativas a $T_{0}$ en una dada dirección pueden escribirse

$$
\frac{\delta T}{T_{0}}(\theta, \phi) \equiv \frac{T(\theta, \phi)-T_{0}}{T_{0}} .
$$

La desviación cuadrática media para estos pequeños apartamientos es

$$
\left\langle\left(\frac{\delta T}{T_{0}}\right)^{2}\right\rangle^{1 / 2}=1.1 \times 10^{-5}
$$

lo cual indica que el FCR es isótropo hasta en una parte en cien mil. Esta isotropía fue confirmada observacionalmente por los distintos experimentos dedicados a medir el FCR. Aumentando esta precisión, aparecen las anisotropías angulares en temperatura que son aquellas que resultan de interés cosmológico.

Las observaciones del FCR enfrentan varios desafíos, como por ejemplo identificar eficazmente la radiación proveniente del Universo temprano y aquella cuyo origen es distinto (fuentes astrofísicas puntuales, radiación de la galaxia, emisión sincrotrón, emisión de granos de polvo, entre otros). Se define como "ruido"o foreground a aquella radiación que se observa en las mismas frecuencias que el FCR pero no se relaciona con la física del Universo primigenio. Por ello, es necesario construir modelos de las emisiones difusas en las mismas longitudes de onda que FCR y restar su efecto. La emisión sincrotrón y el scattering por granos de polvo son las fuentes de contaminación más importantes. La figura 1.2 muestra los mapas del cielo reportados por la Colaboración Planck en distintas frecuencias, los cuales permitirán estudiar, modelar e identificar las contribuciones que no corresponden al FCR en la radiación detectada. 


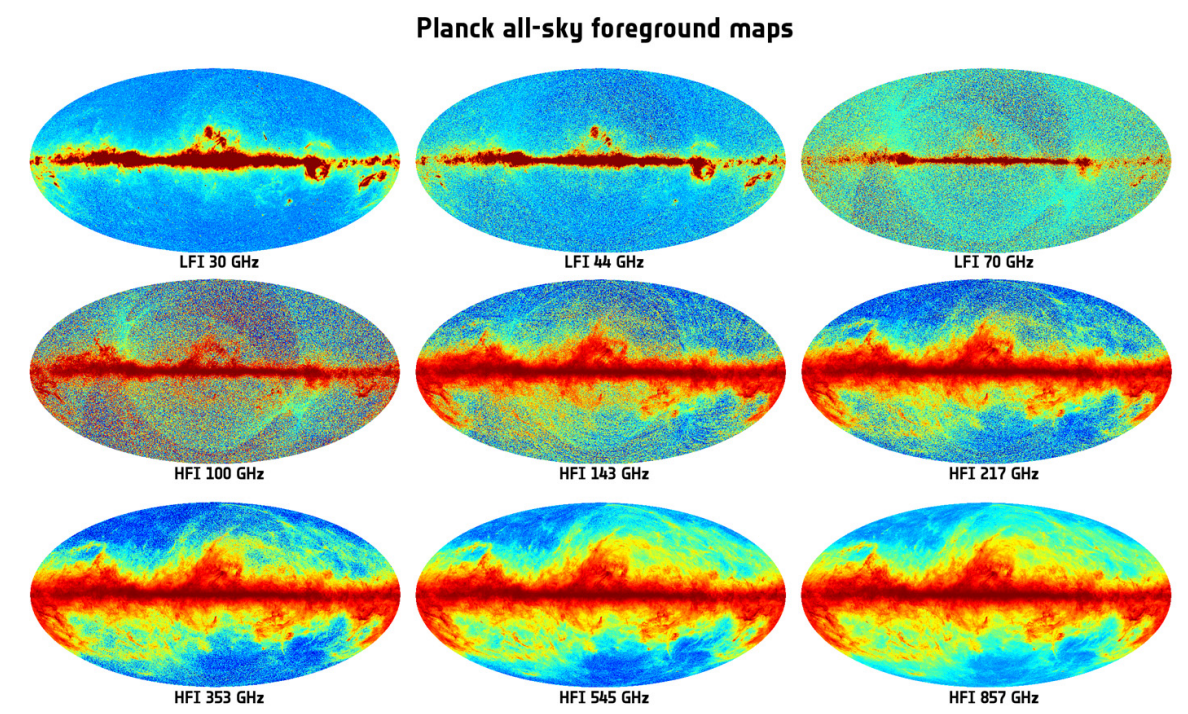

Figura 1.2. Mapas del cielo para las distintas frecuencias dentro de las longitudes de onda de las microondas. Se observa cómo la contribución de la galaxia y de otras fuentes emisoras depende de la frecuencia. Créditos: Planck Collaboration.

\subsection{Formación de átomos neutros en el Universo temprano}

El Universo primordial estaba compuesto por un plasma denso y a gran temperatura donde fotones, protones, electrones y núcleos de elementos químicos livianos se encontraban en una estrecha interacción. Dadas las abundancias relativas, se puede suponer que la componente bariónica del Universo está representada por el hidrógeno (lo cual no es exacto pero resulta una buena aproximación). El mismo puede presentarse en forma de elemento neutro (designado por la letra $H$ ) o como núcleo ionizado (donde se tiene sencillamente un protón $p$ ). Para mantener neutralidad de la carga del Universo, la densidad numérica de electrones libres debe ser igual a la de protones libres: $n_{e}=n_{p}$. El grado de ionización del contenido de materia bariónica en el Universo puede ser expresado del siguiente modo

$$
X \equiv \frac{n_{p}}{n_{p}+n_{H}}=\frac{n_{p}}{n_{B}}=\frac{n_{e}}{n_{B}},
$$

donde $n_{B}$ es la densidad numérica total de materia bariónica. El valor de $X$ va desde 1 cuando la ionización es completa, hasta 0 en el caso de contar con la totalidad de átomos neutros. La energía de ionización del hidrógeno neutro es $13.6 \mathrm{eV}$, es decir que un fotón con energía $h \nu$ mayor es capaz de fotoionizar un átomo de hidrógeno en la siguiente reacción

$$
\mathrm{H}+\gamma \rightarrow \mathrm{p}+\mathrm{e}^{-},
$$

que también puede darse en la dirección opuesta cuando un protón y un electrón se combinan formando un átomo neutro de hidrógeno y liberando un fotón en el proceso

$$
p+e^{-} \rightarrow \mathrm{H}+\gamma .
$$

Si el Universo estuviera compuesto únicamente por fotones, protones y electrones, la fracción de ionización $X$ dependerá del balance entre fotoionización y recombinación radiativa.

Hacia los orígenes del Universo, cuando éste era $10^{-5}$ veces más pequeño de lo que es actualmente, la temperatura de la radiación de fondo era del orden de $T \approx 3 \times 10^{5} \mathrm{~K}$, y la 
energía promedio de los fotones era de $h \nu_{\text {media }} \approx 60 \mathrm{eV}$. Con tanta energía disponible por fotón y con una proporción $n_{\gamma} / n_{B} \sim 2 \times 10^{9}$, la vida media de cualquier átomo de hidrógeno neutro era muy corta, ya que inmediatamente era ionizado por fotones de alta energía. Esto implica que la fracción de ionización del Universo temprano era muy cercana a $X=1$.

En estas condiciones, los fotones interactuaban constantemente con los electrones mediante la dispersión (de ahora en adelante scattering) de Thomson:

$$
\gamma+e^{-} \rightarrow \gamma+e^{-}
$$

Esto significa un intercambio constante de energía y momento entre los fotones y electrones que participaban de la interacción. El camino libre medio para un fotón en estas condiciones es

$$
\lambda=\frac{1}{n_{e} \sigma_{e}},
$$

donde $\sigma_{e}=6.65 \times 10^{-29} \mathrm{~m}^{2}$ es la sección eficaz para el scattering de Thomson. La frecuencia con la que un fotón participa de un proceso de scattering es

$$
\Gamma=\frac{c}{\lambda}=n_{e} \sigma_{e} c
$$

Si el Universo se encuentra completamente ionizado, entonces $n_{e}=n_{p}=n_{B}$.

Los fotones permanecerán acoplados a los electrones mientas la tasa de scattering sea más grande que la velocidad de expansión del Universo, es decir que el camino libre medio $\lambda$ debe ser más pequeño que la distancia de Hubble $1 / H$ (siendo $H$ el factor de Hubble) que caracteriza la expansión. En tanto los fotones sean dispersados frecuentemente por los electrones, ambos permanecerán en equilibrio termodinámico. Esto permite que todo el plasma se encuentre a una misma temperatura $T$.

Se denomina "recombinación" al proceso de formación de átomos neutros de hidrógeno, una vez que el Universo se enfría lo suficiente de modo que resulta más favorable energéticamente que un electrón y un protón se "combinen" para formar un átomo de hidrógeno. Este proceso no es instantáneo si no que toma un determinado tiempo en el cual hay cada vez menos electrones libres para interactuar con la radiación.

Cuando la tasa de scattering de fotones $\Gamma$ cae lo suficiente, los electrones son diluidos por la expansión más rápido de lo que los fotones pueden interactuar con ellos. Este es el momento donde los fotones se desacoplan de los electrones y el Universo se vuelve transparente a la radiación.

\subsection{La física de la recombinación}

Cuando la energía media por fotón es menor que la energía de ionización del hidrógeno $13.6 \mathrm{eV}$, la mayoría de los fotones ya no son capaces de fotoionizar el hidrógeno. Sin embargo, la distribución de energía para los fotones según un cuerpo negro no es constante, si no que prevé una cantidad mucho menor de fotones muy energéticos. Dada la enorme supremacía numérica de los fotones por sobre bariones, es todavía muy probable que siga habiendo ionizaciones. De modo que la temperatura a la que tiene lugar la recombinación no sólo depende de la energía de ionización del átomo de hidrógeno, si no también de la proporción de fotones disponibles para ionizar por cada átomo. 
El valor de $X$ en el Universo temprano está determinado por la reacción

$$
\mathrm{H}+\gamma \leftrightharpoons \mathrm{p}+\mathrm{e}^{-} .
$$

Mientras los fotones estén acoplados a los electrones, esta reacción se mantendrá en equilibrio estadístico. Cuando esto sucede, la relación entre la densidad de partículas $n_{x}$ con masa $m_{x}$ y la temperatura $T$ está dada por la ecuación de Maxwell-Boltzmann

$$
n_{x}=g_{x}\left(\frac{m_{x} k T}{2 \pi \hbar^{2}}\right)^{3 / 2} \exp \left(-\frac{m_{x} c^{2}}{k T}\right),
$$

donde $g_{x}$ es el peso estadístico de las partículas y se cumple $m_{x} c^{2} \gg k T$ (partículas norelativistas). Expresando esta ecuación tanto para la densidad de protones como de electrones y átomos de hidrógeno, y teniendo en cuenta que los fotones tienen una distribución de cuerpo negro, se obtiene

$$
k T_{\text {rec }}=0.323 \mathrm{eV} \Rightarrow \mathrm{T}_{\text {rec }}=3740 \mathrm{~K},
$$

habiendo definido que $X=1 / 2$ en el momento de la recombinación. Esta estimación corresponde a un Universo cuyo redshift es alrededor de $z \approx 1300$. La densidad numérica de electrones libres decae rápidamente durante la época de recombinación y los fotones comienzan a desacoplarse de la materia. Si bien el proceso de desacople no fue instantáneo, tuvo una duración relativamente corta (70 mil años aproximadamente).

La formación de átomos de hidrógeno pasa a ser energéticamente más favorable que la ionización, por lo que la reacción (1.10) ya no se encuentra en equilibrio termodinámico. Los fotones quedan libres de interacciones y viajan casi sin interactuar con otras partículas desde la última vez que fueron dispersados por un electrón. Durante un pequeño intervalo de tiempo $d t$, la probabilidad de que un fotón participe de un proceso de scattering es $d P=\Gamma(t) d t$, donde $\Gamma(t)$ es la tasa de scattering al tiempo $t$. Es decir que si se detectara un fotón del FCR a tiempo $t_{0}$, el número esperado de dispersiones en las que haya participado desde un tiempo $t$ anterior es

$$
\tau(t)=\int_{t}^{t_{0}} \Gamma(t) d t,
$$

y se denomina profundidad óptica de recombinación. El tiempo $t$ para el cual $\tau=1$ corresponde al "último scattering" y representa el tiempo transcurrido desde que un fotón cualquiera del FCR fue dispersado por última vez por un electrón libre. No todos los fotones fueron dispersados por última vez al mismo tiempo, de modo que la "superficie de último scattering" es más bien una cáscara con cierto grosor. Los fotones viajaron a través del Universo disminuyendo su energía progresivamente debido al redshift cosmológico. Actualmente se detectan en las longitudes de onda de las microondas y traen consigo información acerca de la física del Universo en el momento en que se dispersaron por última vez, así como también de la evolución del Universo y el contenido de materia y energía. Al no estar sujeta a la presión de radiación, la materia comienza a estar dominada por la atracción gravitacional. Se forman progresivamente grandes pozos de potencial donde más materia es atraída para formar las grandes estructuras del Universo, como galaxias y cúmulos de galaxias.

\subsection{Observables del Fondo Cósmico de Radiación}

Para comparar modelos cosmológicos alternativos al modelo cosmológico estándar con los datos del Fondo Cósmico de Radiación (FCR) y poner límites a los parámetros libres, 
es necesario contar con mapas del cielo lo más completos posibles y también con la mayor precisión angular disponible. Por ello, dentro de los datos disponibles al momento, la mejor opción que comparte ambas características es el relevamiento de la sonda espacial Planck de la Agencia Espacial Europea (European Space Agency - ESA). La figura 1.3 da cuenta de la resolución de Planck en comparación con las misiones espaciales COBE y WMAP que también han relevado el cielo completo. Además, es muy distinta la metodología para el tratamiento de la radiación contaminante.

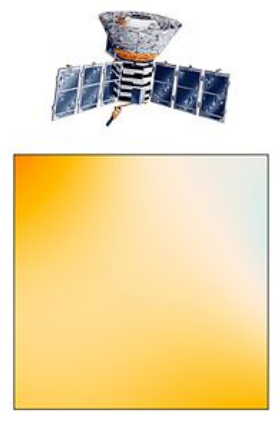

COBE

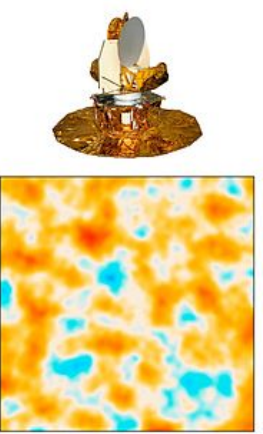

WMAP

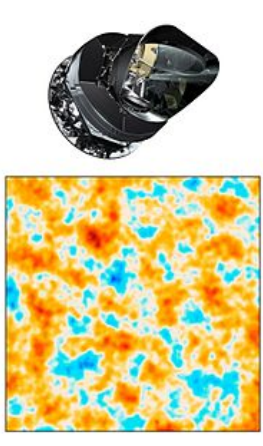

Planck

Figura 1.3. Comparación en la resolución de las mediciones de las misiones espaciales COBE, WMAP y Planck, únicas con relevamiento del cielo completo para las anisotropías del FCR. Créditos: NASA/JPL-Caltech/ESA.

Anteriormente la manera de comparar las observaciones con los modelos cosmológicos propuestos era obtener las predicciones correspondientes y estimar los parámetros propios de la cosmología en relación a un mapa del FCR que se denominaba combinación interlineal. El procedimiento para obtenerlo constaba de combinar las mediciones obtenidas en las distintas bandas de medición del instrumento, restar las fuentes puntuales conocidas, elaborar modelos de los distintos mecanismos de emisión en la galaxia y finalmente interpolar en los puntos cuyos datos se desconocen para completar el mapa. Se obtenía de este modo un mapa del cielo completo con el cual trabajar y una decena de parámetros que ajustar. La propuesta actual es diferente: se realiza de forma conjunta el estudio estadístico del modelo cosmológico junto con los modelos para los foregrounds (radiación contaminante de fondo), de manera que los estudios estadísticos incluyen tanto las predicciones teóricas cosmológicas como los modelos para la radiación proveniente de fuentes contaminantes.

El primer relevamiento de la misión en hacerse público fue en el año 2013 (Planck Collaboration et al., 2014a). El conjunto de datos consta de mediciones para la temperatura realizadas por Planck, datos de polarización reportados por la misión de la NASA WMAP (Bennett et al., 2013), mediciones de temperatura para valores altos de los multipolos (escalas angulares pequeñas) de las colaboraciones ACT (Das et al., 2014) y SPT (Reichardt et al., 2012). A lo largo del capítulo 5 se hará referencia a este conjunto de datos de modo abreviado como Planck2013.

En 2015, la misión Planck hizo público un nuevo relevamiento más completo en polarización y temperatura. Para llevar adelante el presente trabajo, se utilizaron los datos de temperatura para los multipolos $l \geq 30$ (abreviados como PlanckTT) y las mediciones de temperatura y polarización para los multipolos $l<30$ (denominados lowP o lowTEB). A este conjunto de datos se hará referencia de manera abreviada de aquí en adelante como Planck2015. No se incluye polarización a partir del multipolo 30 ya que la colaboración 
Planck indica que aún el análisis y corrección de los datos es levemente dependiente del modelo y no recomienda su utilización para analizar modelos cosmológicos alternativos al modelo cosmológico estándar (Planck Collaboration et al., 2016c).

\subsection{Espectro de temperatura y polarización del FCR}

El campo de radiación del FCR está caracterizado por un tensor de $2 \times 2$

$$
I_{i j}(\hat{n})=\frac{\delta I}{I_{0}}(\hat{n}),
$$

normalizado en términos de la intensidad promedio y donde $\hat{n}=(\sin \theta \sin \varphi, \sin \theta \cos \varphi, \cos \theta)$ representa una dirección en el cielo. El tensor $I_{i j}$ es función de $\hat{n}$ y se definen los versores $\left(\hat{e_{1}}, \hat{e_{2}}\right)$ en el plano perpendicular a $\hat{n}$. La temperatura resulta

$$
T=\frac{I_{11}+I_{22}}{4},
$$

mientras que la polarización se describe a través de los parámetros de Stokes del siguiente modo:

$$
Q=\frac{I_{11}-I_{22}}{4} .
$$

Este último se puede definir operacionalmente como la diferencia de las intensidades que transmiten polarizadores perfectos orientados a lo largo de los ejes $\left(\hat{e_{1}}, \hat{e_{2}}\right)$, mientras que

$$
U=\frac{I_{12}}{2}
$$

es interpretado como la diferencia entre las intensidades de polarizadores perfectos rotados $45^{\circ}$ respecto a los ejes mencionados anteriormente.

Dado que los datos se presentan en una esfera, es natural describirlos a través de una expansión en armónicos esféricos del siguiente modo:

$$
\begin{aligned}
\frac{\delta T(\hat{n})}{T_{0}} & =\sum_{l m} a_{T, l m} Y_{l m}(\hat{n}), \\
(Q+i U)(\hat{n}) & =\sum_{l m} a_{+2, l m+2} Y_{l m}(\hat{n}), \\
(Q-i U)(\hat{n}) & =\sum_{l m} a_{-2, l m-2} Y_{l m}(\hat{n}),
\end{aligned}
$$

donde $(\hat{n})$ corresponde a una determinada dirección en el cielo.

Debido a que las cantidades (1.19) y (1.20) no son invariantes ante rotaciones, es conveniente definir las siguientes cantidades:

$$
\begin{aligned}
E(\hat{n}) & =\frac{1}{2}\left[\bar{\partial}^{2}(Q+i U)-\partial^{2}(Q-i U)\right] \\
& =\sum_{l m}\left[\frac{(l+2) !}{(l-2) !}\right]^{1 / 2} a_{l m}^{E} Y_{l m}(\theta, \varphi), \\
B(\hat{n}) & =\frac{i}{2}\left[\bar{\partial}^{2}(Q+i U)-\partial^{2}(Q-i U)\right] \\
& =\sum_{l m}\left[\frac{(l+2) !}{(l-2) !}\right]^{1 / 2} a_{l m}^{B} Y_{l m}(\theta, \varphi),
\end{aligned}
$$


donde las derivadas de spin tienen la siguiente forma:

$$
\begin{aligned}
& \partial_{s} f(\theta, \phi)=-\sin ^{s}(\theta)\left[\frac{\partial}{\partial \theta}+i \csc (\theta) \frac{\partial}{\partial \phi}\right] \sin ^{-s}(\theta)_{s} f(\theta, \phi), \\
& \bar{\partial}_{s} f(\theta, \phi)=-\sin ^{-s}(\theta)\left[\frac{\partial}{\partial \theta}-i \csc (\theta) \frac{\partial}{\partial \phi}\right] \sin ^{s}(\theta)_{s} f(\theta, \phi),
\end{aligned}
$$

siendo $s=2$ en este caso ya que $Q$ y $U$ son funciones de spin 2 .

Las ecuaciones (1.23) definen las derivadas de spin y una de sus propiedades más importantes es subir o bajar el spin de la cantidad a la cual se aplica la derivada. De esta manera, $E$ y $B$ serán cantidades de spin 0 y por lo tanto invariantes ante rotaciones (Zaldarriaga \& Seljak, 1997). A su vez, debido a su comportamiento diferente ante transformaciones de paridad, $E$ es una cantidad escalar y $B$ una cantidad pseudoescalar. Los nombres $E$ y $B$ han sido elegidos en analogía al campo electromagnético debido al comportamiento de ambas cantidades frente a transformación de paridad.

Como no es posible medir más de una realización del Universo, es imposible realizar estadística con $I, Q$ y $U$. Por ello, se definen las autocorrelaciones y correlaciones cruzadas para la temperatura y los modos $E$ y $B$ de polarización, que son las cantidades que se comparan con las predicciones de los modelos teóricos:

$$
\begin{aligned}
C_{T T}(\xi) & =\left\langle\frac{\delta T}{T_{o}}\left(\hat{n}_{1}\right), \frac{\delta T}{T_{o}}\left(\hat{n}_{2}\right)\right\rangle \\
& =\sum_{l m}\left\langle a^{\star T}{ }_{l m}^{T} a_{l m}^{T}\right\rangle Y_{l m}^{\star}\left(\hat{n}_{1}\right) Y_{l m}\left(\hat{n}_{2}\right) \\
& =\frac{1}{4 \pi} \sum_{l=2}^{\infty}(2 l+1) C_{l}^{T T} P_{l}(\cos \xi), \\
C_{E E}(\xi) & =\left\langle E\left(\hat{n}_{1}\right), E\left(\hat{n}_{2}\right)\right\rangle \\
& =\sum_{l m}\left\langle a^{\star E}{ }_{l m} a_{l m}^{E}\right\rangle Y_{l m}^{\star}\left(\hat{n}_{1}\right) Y_{l m}\left(\hat{n}_{2}\right) \\
& =\frac{1}{4 \pi} \sum_{l=2}^{\infty}(2 l+1) C_{l}^{T E} P_{l}(\cos \xi), \\
C_{B B}(\xi) & =\left\langle B\left(\hat{n}_{1}\right), B\left(\hat{n}_{2}\right)\right\rangle \\
& =\sum_{l m}\left\langle a_{l m}^{\star B} a_{l m}^{B}\right\rangle Y_{l m}^{\star}\left(\hat{n}_{1}\right) Y_{l m}\left(\hat{n}_{2}\right) \\
& =\frac{1}{4 \pi} \sum_{l=2}^{\infty}(2 l+1) C_{l}^{B B} P_{l}(\cos \xi), \\
C_{T E}(\xi) & =\left\langle\frac{\delta T}{T_{o}}\left(\hat{n}_{1}\right), E\left(\hat{n}_{2}\right)\right\rangle \\
& =\sum_{l m}\left\langle a^{\star T}{ }_{l m} a_{l m}^{E}\right\rangle Y_{l m}^{\star}\left(\hat{n}_{1}\right) Y_{l m}\left(\hat{n}_{2}\right) \\
& =\frac{1}{4 \pi} \sum_{l=2}^{\infty}(2 l+1) C_{l}^{B B} P_{l}(\cos \xi),
\end{aligned}
$$

siendo $\xi$ el ángulo en el cielo entre las direcciones $\hat{n}_{1}$ y $\hat{n}_{2}$, y donde $\langle\cdot\rangle$ indica el promedio sobre distintos ensambles. Las correlaciones $C_{E B}(\xi)$ y $C_{B T}(\xi)$ son nulas, debido a que $B$ tiene paridad opuesta a $T$ y $E$.

Las figura 1.4 muestra los resultados obtenidos por la colaboración Planck en las mediciones del espectro de anisotropías angulares para la autocorrelación en la temperatura del FCR. 


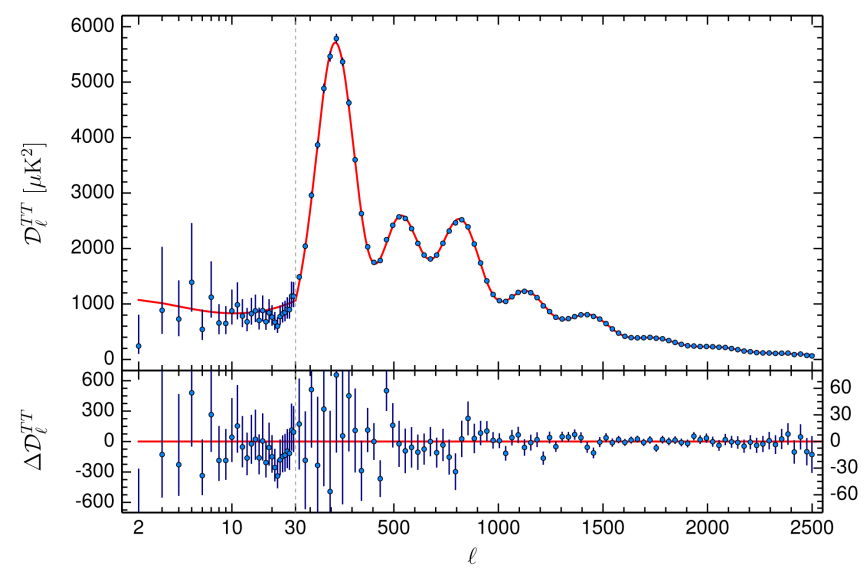

Figura 1.4. Espectro angular de anisotropías de la temperatura del FCR.El eje $x$ es logarítmico hasta $l=30$ y lineal para $30<l<2500$. En el eje $y$, las anisotropías se expresan según $D_{l}^{T T}=l(l+1) C_{l} / 2 \pi$ en unidades de $\mu \mathrm{K}^{2}$. La línea continua corresponde al modelo cosmológico estándar que mejor ajusta a los datos PlanckTT+lowP 2015, mientras que el panel inferior muestra los residuos con respecto a ese modelo. Las barras de error son las incertezas a $\pm 1 \sigma$ (Planck Collaboration et al., 2016b).
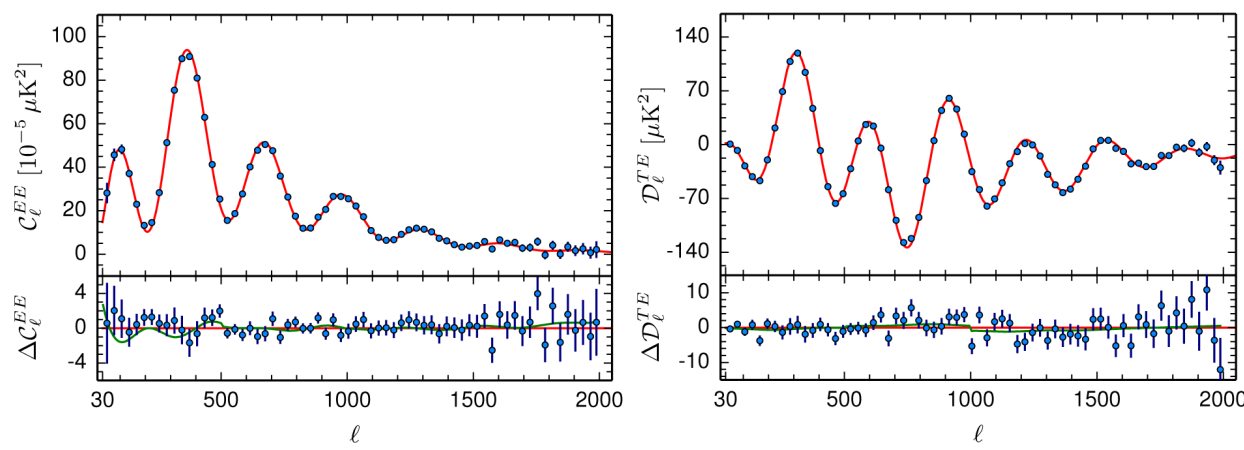

Figura 1.5. Izquierda: Espectro angular del modo E de polarización (panel superior). Derecha: Espectro angular para la correlación cruzada entre los modos E de polarización y la temperatura (panel superior). La línea continua corresponde al modelo cosmológico estándar que mejor ajusta a los datos PlanckTT+lowP 2015. En ambos casos el panel inferior muestra los residuos respecto al modelo de referencia y las barras de error son las incertezas a $\pm 1 \sigma$ (Planck Collaboration et al., 2016b).

El espaciamiento entre los picos indica que las fluctuaciones en el Universo primordial son adiabáticas, mientras que la altura relativa da cuenta de la densidad de materia (Eisentein \& Bennett, 2008). La posición de los picos en el espectro angular permite inferir la curvatura del Universo y la proporción de energía oscura. La figura 1.5 muestra las anisotropías angulares para la autocorrelación del modo E y la correlación cruzada entre temperatura y modo $\mathrm{E}$ (Planck Collaboration et al., 2016b).

\subsection{Oscilaciones Acústicas de Bariones}

Hasta aquí no se ha tenido en cuenta la presencia de materia oscura, que será importante al momento de la formación de estructuras a gran escala. La materia oscura interactúa gravitacionalmente con el plasma. La distribución de materia y radiación no es exactamente homogénea, si no que presenta pequeñas sobredensidades y subdensidades. 
La materia es atraída gravitacionalmente hacia las regiones más densas, por lo que la densidad en estas regiones tenderá a crecer. En contrapartida, la presión de radiación comienza a aumentar (recordar que materia y radiación permanecen aún acopladas), poniendo un límite al crecimiento de la sobredensidad. Esta genera a su vez un exceso de presión de radiación que desencadena la propagación de ondas de sonido a través del plasma.

Luego del desacople, la propagación de ondas acústicas cesa, dejando una huella característica tanto en el FCR como en la distribución de materia.

Sea la inhomogeneidad relativa de densidad (de bariones, fotones, neutrinos o materia oscura) en una determinada posición $\vec{x}$ :

$$
\delta(\vec{x})=\frac{\rho(\vec{x})-\bar{\rho}}{\bar{\rho}},
$$

donde $\bar{\rho}$ es la densidad promedio. La figura 1.6 muestra esquemáticamente la evolución de una sobredensidad inicial a un determinado tiempo $t=0$. La componente de materia oscura permanece relativamente cerca del pico inicial mientras que radiación y materia bariónica se propagan acoplados según la onda expansiva. Alrededor de $z \sim 1100$, los fotones y la materia se desacoplan. Los primeros se propagan casi sin interacción y conforman lo que actualmente se observa como el FCR, mientras que los bariones comienzan a caer en los pozos de potencial conformados por la materia oscura y viceversa. Luego del desacople las sobredensidades estarán separadas por la máxima distancia $r_{s}$ que viajó la onda de sonido desde el Big Bang, y son las que luego evolucionarán en galaxias y cúmulos de galaxias, separados por una distancia característica que se estima estadísticamente analizando grandes cartografiados de galaxias.
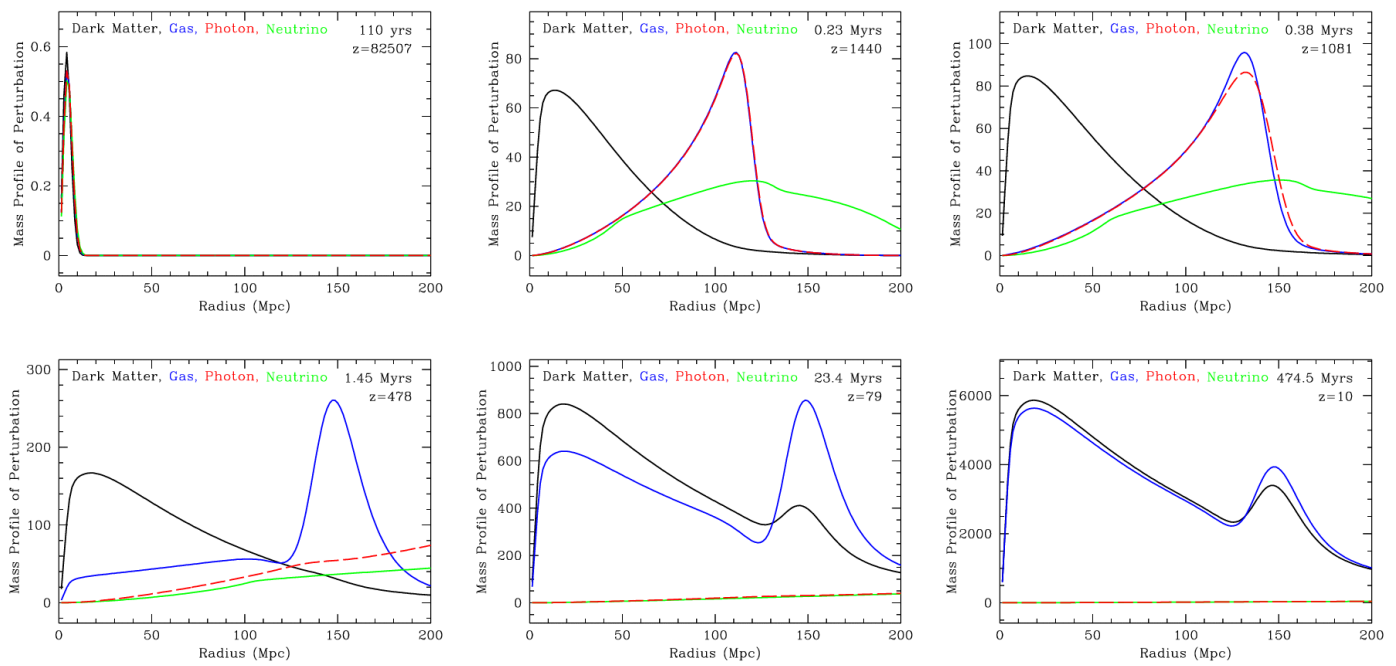

Figura 1.6. Evolución de una sobredensidad inicial de materia oscura, bariones y fotones partiendo de un tiempo inicial arbitrario. Bariones y fotones se propagan acoplados según una onda acústica, mientras que la materia oscura se va expandiendo, pero permanece una sobredensidad en el punto inicial. Luego del desacople, los fotones se distribuyen uniformemente por todo el espacio, mientras que los bariones caen en los pozos de potencial formados por la materia oscura y ésta a su vez interactúa gravitacionalmente con la materia bariónica viéndose atraída hacia aquellos puntos donde hubiera sobredensidades. Como consecuencia, la formación de estructura a gran escala se presenta con una separación estadística característica, que se busca medir con los grandes catálogos de galaxias. Créditos de la imagen: Daniel Eisenstein - Sloan Digital Sky Survey. 
El Universo temprano no presenta una única inhomogeneidad, sino una distribución de sobredensidades y subdensidades. La propagación de las ondas de sonido determina regiones esféricas alrededor de regiones más densas que se solapan entre sí, lo mismo ocurre son las regiones de menor densidad.

Se describe a $\delta(\vec{x})$ como una variable aleatoria gaussiana cuya distribución en $\vec{x}$ constituye un campo correlacionado, caracterizado por su función de correlación o espectro de potencias. Para que este tratamiento tenga validez es necesario que las inhomogeneidades relativas sean pequeñas: $|\delta \rho| / \rho \ll 1$. Las sobredensidades sobre estas cáscaras esféricas no pueden ser determinadas individualmente, pero se puede estimar estadísticamente una separación representativa. Se espera que aparezca un pico en la función de correlación

$$
\xi(\vec{s})=\langle\delta(\vec{x}) \delta(\vec{x}+\vec{s})\rangle_{\vec{x}}
$$

a una distancia $r_{s}$ caracterizada por la propagación de la onda de sonido al momento del desacople entre materia y radiación, conocida como el horizonte de sonido al momento de la recombinación (figura 1.7).

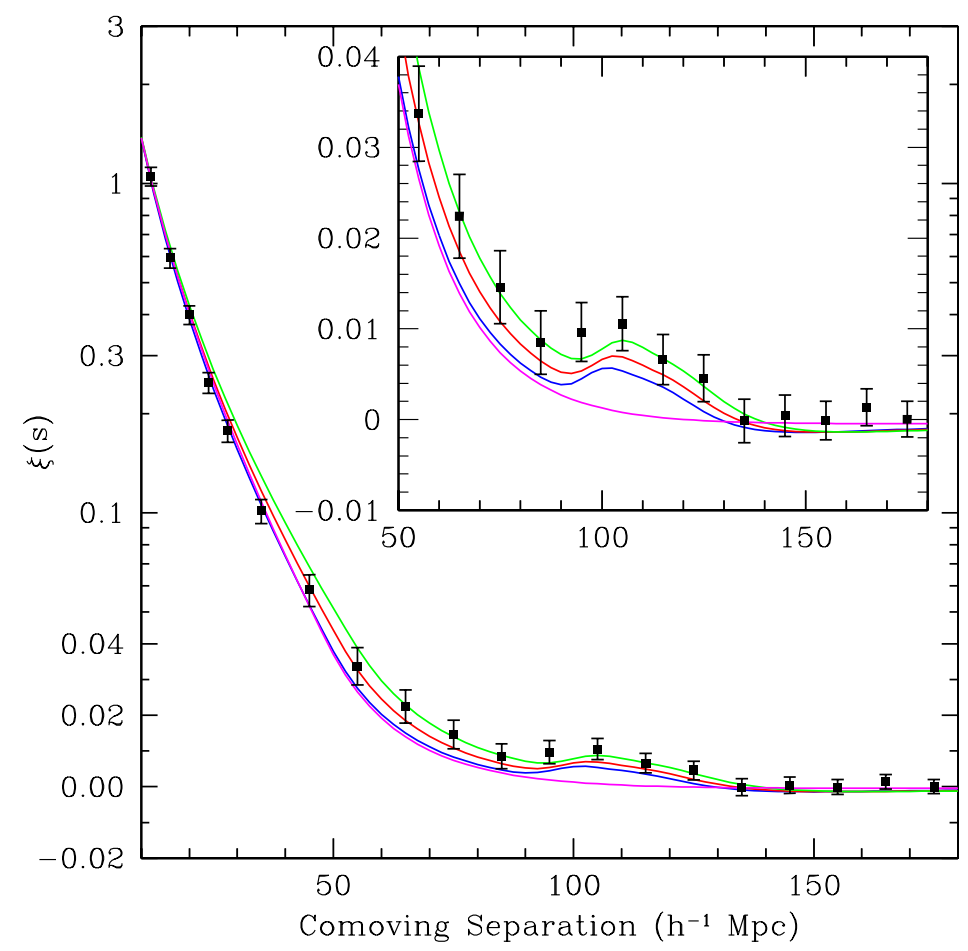

Figura 1.7. Se conoce como "pico BAO" al exceso de correlación que se observa en la distribución de galaxias, dando cuenta de una separación característica en la distancia comóvil entre dos sobredensidades determinadas estadísticamente. Las líneas corresponden a modelos cosmológicos estándar con distintos valores para la componente de materia en el Universo. Figura: Eisenstein et al. (2005).

\subsubsection{Observables de las Oscilaciones Acústicas de Bariones}

Las observaciones de las Oscilaciones Acústicas de Bariones (BAO por sus siglas en inglés) implican medir la función de correlación $\xi(\vec{s})$ o, su equivalente en el espacio de Fourier, el espectro de potencias $P(k)$. Esto se realiza mediante relevamientos tridimensionales de la 
posición de objetos astrofísicos (denominados "trazadores") que dan cuenta de la distribución total de materia, entre los que se cuentan galaxias, quásares, LRG (Luminous Red Galaxies), etc. Si bien los relevamientos actuales son cada vez más amplios y profundos, debido a las limitaciones naturales en la observación, los redshift de estas muestras suelen ser mucho menores que uno, restringiéndose la mayor cantidad de datos al Universo local. De allí la importancia de complementar los datos del FCR con el cartografiado de galaxias: proveen información acerca de un mismo proceso físico pero se basan en observables completamente distintos.

Dado un determinado redshift, la función de correlación puede ser medida a lo largo de la línea de la visual y perpendicular a ella. En el primer caso, la posición del pico BAO corresponde a una separación en redshift:

$$
\Delta z=r_{s} H(z) / c
$$

donde $H(z)$ es el parámetro de Hubble. En la dirección perpendicular, la posición del pico se corresponde con un ángulo

$$
\Delta \theta(z)=\frac{r_{s}}{(1+z) D_{A}(z)},
$$

donde $D_{A}(z)$ es la distancia de diámetro angular:

$$
D_{A}(z)=\frac{1}{1+z} \int_{0}^{z} \frac{c d z^{\prime}}{H\left(z^{\prime}\right)}
$$

De la medición de las separaciones angulares y longitudinales se obtienen los indicadores de interés $D_{A}(z) / r_{s}$ y $r_{s} \times H(z)$. Sin embargo, es difícil obtener observacionalmente estas medidas por separado, de modo que la cantidad que se estima a partir de los catálogos de galaxias es la distancia de volumen promedio:

$$
D_{v}(z)=\left[(1+z) D_{A}(z)\right]^{2 / 3}[c z / H(z)]^{1 / 3} .
$$

\subsubsection{Datos utilizados para BAO}

En lo que respecta a esta tesis, se utilizan datos de las oscilaciones acústicas de bariones en conjunto con los datos de temperatura y polarización de Planck (Planck Collaboration et al., 2016c). Los datos utilizados comprenden los siguientes relevamientos: 6dF Galaxy Survey (6dFGS) (Beutler et al., 2011), Sloan Digital Sky Survey Main Galaxy Sample (SDSSMGS) (Ross et al., 2015), BOSS galaxy samples (Gil-Marín et al., 2016), LOWZ y CMASS (Anderson et al., 2014). En el capítulo 5 se referirá a este conjunto de datos con el nombre de BAO.

\subsection{Telescopio Espacial Hubble}

De la variedad de experimentos dedicados a estimar los parámetros de los modelos cosmológicos, muchos de ellos han logrado buen acuerdo entre los datos permitiendo restringir aún más el espacio de parámetros. Sin embargo, para la estimación del valor actual de la constante de Hubble existe una discrepancia a más de $5 \sigma$ entre las estimaciones realizadas a partir de los datos del FCR y los métodos que utilizan cefeidas, supernovas tipo Ia, paralajes y binarias eclipsantes. 
La colaboración Planck reporta para sus datos PlanckTT+lowP (Planck Collaboration et al., 2016b):

$$
H_{0}=67.31 \pm 0.96 \mathrm{~km} \mathrm{~s}^{-1} \mathrm{Mpc}^{-1},
$$

mientas que las mediciones llevadas a cabo con el Telescopio Espacial Hubble (Hubble Space Telescope - HST) estiman (Riess et al., 2016):

$$
H_{0}=73.24 \pm 1.74 \mathrm{~km} \mathrm{~s}^{-1} \mathrm{Mpc}^{-1} .
$$

Esta diferencia es denominada en la literatura como tensión en la estimación de $H_{0}$, ya que ambas determinaciones (cada una con su precisión) pueden estimar una misma variable a través de distintos métodos y no son compatibles estadísticamente.

En el capítulo 5 se referirá al valor de $H_{0}$ obtenido por el Hubble Space Telescope con el nombre HST. 


\section{Capítulo 2}

\section{Análisis Estadístico}

Las primeras secciones de este capítulo están basadas en la tesis doctoral de C. Scóccola, mientras que la sección de evidencia bayesiana se basa en el trabajo de Benetti et al. (2016).

\subsection{Introducción}

Dado un modelo teórico determinado, es importante establecer su validez contrastando sus predicciones con las observaciones de la naturaleza. En el caso de los modelos de colapso, las predicciones teóricas de los observables se describen en el capítulo 4, mientras que los observables utilizados en esta tesis se describen en el capítulo 1.

En estadística se utilizan muestras de datos para hacer inferencias acerca de un modelo probabilístico, pudiendo determinar su viabilidad y estimar sus parámetros libres. Existen dos enfoques principales para abordar el análisis que se denominan frecuentista y bayesiano.

El enfoque frecuentista interpreta la probabilidad como la frecuencia de aparición de un determinado resultado en una experiencia que se repite, siendo la estimación de parámetros y los test estadísticos las herramientas más importantes para este enfoque. Permite informar objetivamente acerca del resultado de un experimento sin necesidad de incorporar conocimientos previos respecto al parámetro o la teoría sometidos a análisis.

La estadística bayesiana, en cambio, ofrece una interpretación más general de la probabilidad e incluye un grado de conocimiento previo. Por ejemplo, se puede expresar el grado de conocimiento acerca del valor verdadero de un parámetro mediante la función de densidad de probabilidad del mismo. Es decir que los métodos bayesianos permiten incorporar de manera natural información adicional como restricciones físicas o información proveniente de otros datos, requiriendo la imposición de una probabilidad a priori que luego se irá actualizando en el transcurso del análisis estadístico.

Para muchos problemas de inferencia, ambos enfoques pueden arribar a los mismos resultados numéricos aún habiéndose basado sobre interpretaciones diferentes de la probabilidad. Sin embargo, si la muestra de datos es reducida o se está midiendo un parámetro cerca de un límite físico, los distintos enfoques pueden arribar a resultados diferentes y es necesario optar por uno de ellos. En la literatura, se adopta el enfoque bayesiano para el estudio de los modelos cosmológicos. Continuar con este enfoque no sólo permitirá contrastar los resultados obtenidos con los de otros autores, si no que también pone a disposición una gran variedad de herramientas estadísticas utilizadas por la comunidad científica, permitiendo analizar 
distintos modelos bajo un mismo protocolo de actuación de estadística.

\subsection{Enfoque bayesiano}

La perspectiva bayesiana no distingue entre observables y parámetros de un modelo estadístico, si no que todos son considerados cantidades desconocidas y se les puede asociar un concepto de probabilidad. Si $D$ son los datos observados y $\theta$ los parámetros del modelo más los datos faltantes, se puede construir una distribución de probabilidad conjunta $P(D, \theta)$ sobre todas las cantidades aleatorias más las desconocidas.

Esta distribución conjunta consta de dos partes: una distribución de probabilidad a priori $P(\theta)$ y una función likelihood $P(D \mid \theta)$ que expresa, luego de conocido el resultado de un experimento, qué tan probable era obtener ese resultado. Expresar ambas cantidades describe un modelo probabilístico completo, donde la relación entre ambas está dada por

$$
P(D, \theta)=P(D \mid \theta) P(\theta) .
$$

El teorema de Bayes determina la distribución a posteriori de $\theta$ :

$$
P(\theta \mid D)=\frac{P(\theta) P(D \mid \theta)}{\int P(\theta) P(D \mid \theta) d \theta},
$$

que es la distribución de los parámetros o datos $\theta$ condicionada a las observaciones $D$ y normalizada. Tomando este resultado como punto de partida, pueden calcularse los valores esperados a posteriori en cualquier función $f(\theta)$ :

$$
E[f(\theta) \mid D]=\frac{\int f(\theta) P(\theta) P(D \mid \theta) d \theta}{\int P(\theta) P(D \mid \theta) d \theta} .
$$

El objetivo principal del enfoque bayesiano es inferir estas cantidades, pero también una de las máximas dificultades. La integración sobre las probabilidades en un amplio espacio de parámetros y con gran cantidad de datos disponibles constituye la principal problemática para obtener resultados en este marco. La propuesta para llevar adelante la evaluación de este tipo de expresiones es a través del método de las cadenas de Markov-Monte Carlo.

\subsection{Cadenas de Markov-Monte Carlo}

En la teoría de probabilidad se conoce como cadena de Markov a un tipo especial de proceso estocástico discreto en el que la probabilidad de que ocurra un evento depende únicamente del evento inmediatamente anterior. El método de Monte Carlo es un método no determinista utilizado para aproximar expresiones matemáticas complejas y costosas de evaluar con exactitud. Las cadenas de Markov-Monte Carlo comprenden una serie de algoritmos para elaborar el muestreo de una distribución de probabilidad. Se construye una cadena de Markov cuya distribución de equilibrio ${ }^{1}$ sea la distribución deseada, luego de observar la cadena tras un número de pasos se obtiene una muestra de esta distribución. Cuantos mayor sea la cantidad de pasos, mejor se representará la distribución buscada. Las cadenas de Markov-Monte Carlo constan de dos partes: la cadena de Markov y la integración Monte Carlo.

\footnotetext{
${ }^{1}$ La distribución de equilibrio es aquella tal que permite a una cadena converger a su distribución estacionaria (independiente del tiempo) indistintamente de su punto de partida.
} 


\subsubsection{Integración Monte Carlo}

La integración Monte Carlo evalúa $E[f(X)]$ tomando muestras $\left\{X_{i} ; i=1, \ldots, n\right\}$ a partir de la función $P(\theta \mid D)$ y luego se hace la aproximación:

$$
E[f(X)] \simeq \frac{1}{n} \sum_{i=1}^{n} f\left(X_{i}\right)
$$

Así, la media de la población se estima con la media de la muestra. Si las muestras $\left\{X_{i}\right\}$ son independientes, la ley de los grandes números ${ }^{2}$ establece que la aproximación se hace cada vez más precisa al aumentar $n$. Es importante tener presente que $n$ no es el número de datos, si no que representa el tamaño de la muestra (la cual puede controlarse durante el análisis).

Tomar muestras $\left\{X_{i}\right\}$ a partir de $P(\theta \mid D)$ no siempre es posible dado que esta función puede no ser común. Sin embargo, puede generarse por cualquier proceso que tome muestras dentro de su soporte en las proporciones correctas. Una manera de hacerlo es a través de una cadena de Markov que tenga a $P(\theta \mid D)$ como distribución estacionaria, que es lo que se conoce como cadena de Markov-Monte Carlo.

\subsubsection{Cadenas de Markov}

Una cadena de Markov es una secuencia de variables aleatorias $\left\{X_{0}, X_{1}, X_{2}, \ldots\right\}$ tales que para $n \geq 0$ el siguiente estado $X_{i+1}$ se muestrea de una distribución $P\left(X_{i+1} \mid X_{i}\right)$ que dependa sólo del estado actual de la cadena $X_{i}$, donde $P(\cdot \mid \cdot)$ es el kernel de transición de la cadena. A medida que avanza, la cadena perderá progresivamente información del estado inicial $X_{0}$ y la distribución de la cual se muestrean los valores irá tendiendo a una distribución estacionaria $\phi(\cdot)$.

Luego de un tiempo de burn in lo suficientemente largo la cadena logra estabilizarse (ver figura 2.1), por ejemplo transcurrida una $j$ cantidad iteraciones; los puntos $\left\{X_{i} ; i=j+\right.$ $1, \ldots, n\}$ se extraerán de la distribución $\phi(\cdot)$. Es necesario asegurar que la cadena construida es tal que su distribución estacionaria $\phi(\cdot)$ coincide con la distribución a posteriori $P(\theta \mid D)$. Un método posible es utilizar el algoritmo de Metropolis-Hastings.

Una vez descartadas las muestras de burn in, el estimador de $E[f(X)]$ se calcula

$$
\bar{f}=\frac{1}{n-j} \sum_{i=j+1}^{n} f\left(X_{i}\right),
$$

que se denomina promedio ergódico. La convergencia al valor de expectación requerido se asegura mediante el teorema ergódico.

\subsubsection{Algoritmo de Metropolis-Hastings}

En estadística, el algoritmo de Metropolis-Hastings es un método particular de cadenas de Markov-Monte Carlo para obtener una secuencia de muestras aleatorias de una distribución de probabilidad cuyo muestreo directo resulta dificultoso. Esta secuencia puede utilizarse

\footnotetext{
${ }^{2}$ En la teoría de la probabilidad, la ley débil de los grandes números establece que si una sucesión infinita de variables aleatorias independientes $X_{1}, X_{2}, X_{3}, \ldots$ tales que su valor esperado $\mu$ y varianza $\sigma^{2}$ son los mismos, entonces el promedio $\bar{X}_{n}=\left(X_{1}+\ldots+X_{n}\right) / n$ converge a $\mu$. Por lo tanto, para cualquier número positivo $\varepsilon$ se obtiene $\lim _{\infty} P\left(\left|\bar{X}_{n}-\mu\right|<\varepsilon\right)=1$.
} 

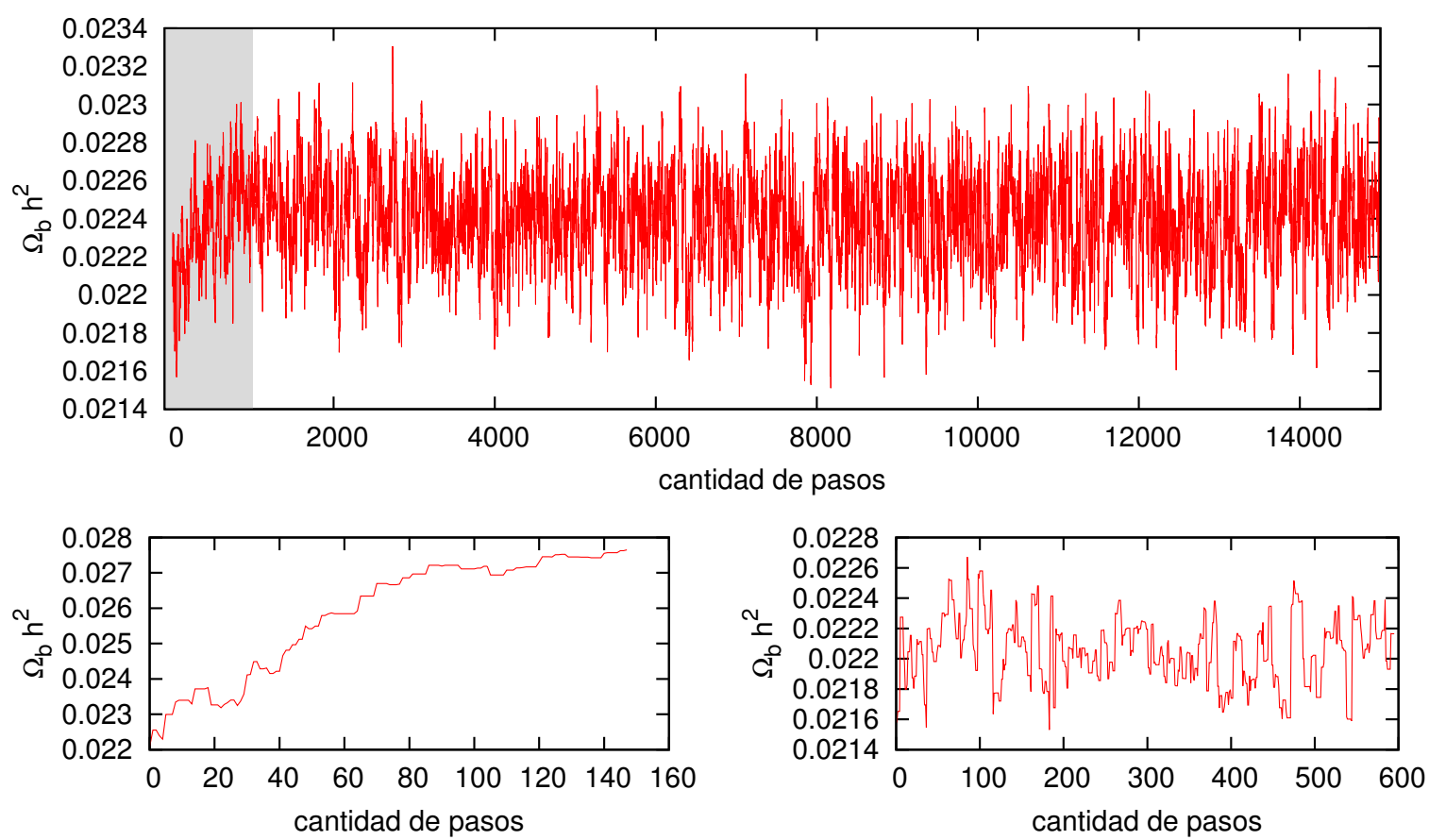

Figura 2.1. Ejemplos de Cadenas de Markov Montecarlo en el estudio estadístico del parámetro $\Omega_{b} h^{2}$. La figura superior muestra una cadena estabilizada luego de un período de burn in, que se encuentra sombreado en gris. Las figuras inferiores muestran dos ejemplos de cadenas que no han logrado estabilizarse y que, por tanto, no resultan adecuadas para hacer inferencias acerca del valor del parámetro en cuestión.

para aproximar la distribución o calcular una integral. Este método es particularmente utilizado para explorar distribuciones multidimensionales especialmente cuando el número de dimensiones es elevado (como en el caso de los parámetros cosmológicos).

Dado un paso $i$, este algoritmo elige el siguiente estado $X_{i+1}$ muestreando un punto candidato $Y$ de la distribución propuesta $q\left(\cdot \mid X_{t}\right)$, que puede ser por ejemplo una distribución normal de múltiples variables con valor medio $X$ y una matriz fija de covarianza. Se calcula para el punto candidato $Y$ la probabilidad $\alpha\left(X_{i}, Y\right)$ tal que

$$
\alpha(X, Y)=\min \left(1, \frac{P(Y) q(X \mid Y)}{P(X) q(Y \mid X)}\right) .
$$

Luego se elige un número aleatorio $0<x_{r n d}<1$ que se compara con la probabilidad obtenida para determinar la elección del siguiente estado:

$$
\begin{aligned}
\text { si } x_{r n d} & <\alpha\left(X_{i}, Y\right) \Rightarrow X_{i+1}=Y, \\
\text { si en cambio } x_{r n d} & >\alpha\left(X_{i}, Y\right) \Rightarrow X_{i+1}=X_{i},
\end{aligned}
$$

y se incrementa $i$. La distribución propuesta $q(\cdot \mid \cdot)$ puede tener cualquier forma, mientras que la distribución estacionaria de la cadena será $P(\theta \mid D)$.

\subsubsection{Convergencia}

En las simulaciones de cadenas de Markov-Monte Carlo siempre habrá regiones del espacio de parámetros que no serán exploradas por una cadena de longitud finita, por lo que hay 
que establecer un criterio que determine si es representativa del experimento la muestra recorrida. Comparar distintas simulaciones y establecer un criterio de convergencia otorga predictibilidad al método; si no se establece convergencia entre las cadenas, nada puede concluirse. También es importante comenzar cada simulación independiente desde un punto de inicial distinto, eliminando así cualquier tipo de vicios.

El modo recomendado para monitorear la convergencia se basa en detectar cuándo las cadenas de Markov han perdido por completo la referencia de sus puntos de partida, comparando distintas secuencias iniciadas desde diferentes puntos de partida y corroborando que sean indistinguibles.

Una forma de establecer un criterio cuantitativo consiste en analizar las varianzas, diagnosticando una convergencia aproximada cuando la varianza entre diferentes cadenas no es mayor a la varianza dentro de una secuencia individual. Paralelamente, también se puede monitorear de manera independiente otros indicadores de interés o "resúmenes escalares" $\psi$, como los parámetros de la distribución. Por ejemplo, tomando $m$ simulaciones paralelas de longitud $n$ cada una, se busca una manera cuantitativa de determinar si existe un apartamiento mayor de lo esperado respecto a su variabillidad interna. Sean $\psi_{i j}$ con $1 \leq i \leq m \mathrm{y}$ $1 \leq j \leq n$ los eslabones de la cadena. Se define la varianza $B$ entre cadenas y la varianza $W$ dentro de las cadenas del siguiente modo:

$$
\begin{aligned}
B & =\frac{n}{m-1} \sum_{i=1}^{m}\left(\bar{\psi}_{i}-\bar{\psi}\right)^{2}, \\
W & =\frac{1}{m} \sum_{i=1}^{m} s_{i}^{2},
\end{aligned}
$$

donde

$$
\begin{aligned}
\bar{\psi}_{i} & =\frac{1}{n} \sum_{j=1}^{n} \psi_{i j}, \\
\bar{\psi} & =\frac{1}{m} \sum_{i=1}^{m} \bar{\psi}_{i}, \\
s_{i}^{2} & =\frac{1}{n-1} \sum_{j=1}^{n}\left(\psi_{i j}-\bar{\psi}_{i}\right)^{2} .
\end{aligned}
$$

Las cantidades $B$ y $W$ permiten construir dos estimadores de la varianza de $\psi$. Uno de ellos

$$
\widehat{\operatorname{var}}(\psi)=\frac{n-1}{n} W+\frac{1}{n} B
$$

el cual no tiene sesgo si los puntos iniciales de las simulaciones fueron verdaderamente extraídos de la distribución $P(\theta \mid D)$, pero termina siendo sobre-estimado si los puntos iniciales se encuentran apropiadamente dispersos. Por otro lado, dado cualquier número finito $n$ la varianza $W$ dentro de la secuencia debe subestimar la varianza de $\psi$, siendo que las secuencias individuales no han tenido tiempo de recorrer toda la distribución y tendrán, por ende, menor variabilidad. En el límite $n \rightarrow \infty$ tanto $\widehat{\operatorname{var}}$ como $W$ deben acercarse a $\operatorname{var}(\psi)$ pero desde direcciones opuestas.

El estimador $R$ está definido como

$$
\sqrt{R}=\sqrt{\frac{\widehat{\operatorname{var}}(\psi)}{W}},
$$


y se acerca a 1 a medida que la secuencia converge, indicando que las cadenas de Markov se están superponiendo. Por ende, se considera que has cadenas han convergido cuando el valor de $R \sim 1$. En los estudios realizados en este trabajo, se toma como criterio de convergencia la restricción $R-1<0.02$.

\subsection{Función de Likelihood para el FCR}

La función de likelihood utilizada para el análisis de los modelos cosmológicos es aquella que compara las anisotropías del FCR con los datos y es provista por cada una de las colaboraciones e integrada a los códigos numéricos que implementan las cadenas de Markov-Monte Carlo. Suponiendo que la señal de la temperatura (o polarización) en cierta dirección del cielo es una muestra de una distribución gaussiana, y dadas $N_{p}$ medidas en distintas direcciones, la función likelihood $L$ para el FCR se podría resumir del siguiente modo:

$$
L=\frac{1}{(2 \pi)^{N_{p} / 2}(\operatorname{det} C)^{1 / 2}} \exp \left(-\frac{1}{2} \Delta C^{-1} \Delta\right),
$$

donde $\Delta$ es el vector de dimensión $N_{p}$ que contiene los datos y $C$ es la matriz de covarianza total (suma entre la matriz de covarianza del ruido y la matriz de covarianza de la señal).

\subsubsection{Evidencia Bayesiana}

Una vez estimados los parámetros a través de un método estadístico adecuado, resulta de interés la comparación entre distintos modelos que describan un mismo fenómeno, los cuales no siempre cuentan con la misma cantidad de parámetros libres.

El modelo de comparación de Bayes es una herramienta que recompensa a los modelos que ajustan bien a los datos demostrando fuerte predictibilidad, mientras que penaliza a los modelos con mayor número de parámetros que no son estrictamente requeridos por los datos. La evidencia bayesiana $\mathcal{E}$ se define como la likelihood marginal para el modelo $M_{i}$ :

$$
\mathcal{E}_{M_{i}}=\int P\left(D \mid \theta, M_{i}\right) \pi\left(\theta \mid M_{i}\right) d \theta,
$$

donde $\pi\left(\theta \mid M_{i}\right)$ es la función de distribución de probabilidad a priori. La comparación de la evidencia bayesiana entre dos modelos se da a través del factor de Bayes, definido como:

$$
\mathcal{B}_{i j}=\frac{\mathcal{E}_{M_{i}}}{\mathcal{E}_{M_{j}}}
$$

siendo $M_{j}$ el modelo de referencia. Cuanto más complejo el modelo $M_{i}$ (por ejemplo con mayor cantidad de parámetros con respecto al modelo de referencia) mayor será la likelihood, pero la evidencia favorecerá el modelo más simple si ambos ajustan con la misma precisión a los datos a través de un volumen menor de la probabilidad a priori.

Si se asumen probabilidades a priori uniformes para cada parámetro, tales que pueda escribirse $\pi(\theta \mid M)=\left(\Delta \theta_{1} \cdots \Delta \theta_{n}\right)^{-1}$, se tiene que

$$
\mathcal{B}_{i j}=\frac{\int P\left(D \mid \theta, M_{i}\right)\left(\Delta \theta_{1} \cdots \Delta \theta_{n_{i}}\right)^{-1} d \theta}{\int P\left(D \mid \theta^{\prime}, M_{j}\right)\left(\Delta \theta_{1}^{\prime} \cdots \Delta \theta_{n_{j}}^{\prime}\right)^{-1} d \theta} .
$$

La escala usual para juzgar diferencias en la evidencia de los distintos modelos es la escala de Jeffreys (Jeffreys, 1961; Trotta, 2007) mostrada en la tabla 2.1, que otorga niveles de significancia calibrados empíricamente para el grado de evidencia. El caso en que $\ln \mathcal{B}_{i j}<0$ indica preferencia por el modelo de comparación. 


\begin{tabular}{cccc}
$\ln \mathcal{B}_{i j}$ & Posibilidades & Probabilidad & Descripción \\
\hline$<1$ & $<3: 1$ & $<0.750$ & inconclusa \\
1 & $\sim 3: 1$ & 0.750 & evidencia débil \\
2.5 & $\sim 12: 1$ & 0.923 & evidencia moderada \\
5 & $\sim 150: 1$ & 0.993 & evidencia fuerte
\end{tabular}

Tabla 2.1. Indicaciones de evidencia de probabilidad para la comparación de un determinado modelo utilizando otro como referencia según el factor de Bayes.

\subsection{Códigos numéricos}

La mejor manera para determinar el valor de los parámetros libres de un modelo sería, idealmente, probar todas las combinaciones posibles de ellos y ver cuál ajusta mejor a los datos utilizando, por ejemplo, un test de mínimos cuadrados. Bastaría con dividir el espacio de parámetros en una grilla y tomar valores representativos para cada intervalo multidimensional.

Sin embargo, este tipo procedimiento resulta completamente inviable para determinar parámetros libres de modelos cosmológicos. El tiempo de cálculo sería de meses y hasta años aún con los mejores equipos de cómputo disponibles. Es por ello que se utilizan métodos estadísticos para estimar estos valores y con ello reducir el tiempo de cálculo a unos pocos días o semanas.

Las cadenas de Markov-Monte Carlo han sido implementadas para la determinación de parámetros cosmológicos en el código CosmoMC ${ }^{3}$ (Lewis \& Bridle, 2002), que es un código abierto, libre y utilizado por la comunidad científica. El lenguaje de programación es el fortran y consta de dos partes: el programa base que implementa los algoritmos descriptos en las secciones anteriores, y el getdist que analiza la convergencia de las cadenas y calcula las estimaciones de los parámetros. Este último también brinda la oportunidad de construir la información necesaria para poder graficar probabilidades a posteriori y contornos de confianza. En los últimos años, este último código ha migrado paulatinamente al lenguaje python.

Las funciones likelihood son construidas para cada conjunto de datos por la colaboración que realizó el experimento. Una vez que los datos son liberados para uso público, se incorpora la likelihood correspondiente al código CosmoMC para habilitar su implementación dentro del análisis estadístico. El conjunto de datos utilizados para estimar los parámetros cosmológicos es precisado por el usuario en la ación inicial.

En cuanto a las predicciones teóricas, éstas son calculadas por un código independiente cuya ejecución se convoca a cada paso de las cadenas. El CAMB ${ }^{4}$ (Lewis et al., 2000) calcula las predicciones teóricas de los observables del FCR y también está escrito en lenguaje fortran. $\mathrm{Al}$ igual que el CosmoMc, es de código libre y abierto, lo que permite su modificación para implementar los cambios necesarios.

Como configuración de partida, el CAMB recibe un conjunto de valores fijos para los parámetros del modelo cosmológico. Integra numéricamente las ecuaciones diferenciales acopladas que describen el estado del universo previo al desacople y su evolución posterior hasta el momento presente, calculando los observables teóricos para los coeficientes de las anisotropías angulares del FCR. Esto implica tratar con dos tipos de partículas: relativistas y no relativistas. Las ecuaciones de estado que describen la dinámica del plasma primordial estarán

\footnotetext{
${ }^{3}$ Cosmological Monte Carlo

${ }^{4}$ Code for Anisotropies in the Microwave Background
} 
representadas por las ecuaciones de Einstein y las ecuaciones de Boltzmann (Ma \& Bertschinger, 1995; Seljak \& Zaldarriaga, 1996). Ambas están a su vez acopladas con la métrica de fondo y las perturbaciones. Dado un conjunto de valores para los parámetros cosmológicos y ciertas suposiciones respecto a las condiciones del modelo, el CAMB permite resolver numéricamente las ecuaciones de estado acopladas para dar como resultado el espectro angular de anisotropías del FCR, que es susceptible de comparación directa con los datos.

Una vez realizado satisfactoriamente el análisis estadístico mediante las cadenas de MarkovMonte Carlo, se calcula la evidencia bayesiana con el código MultiNest (Feroz \& Hobson, 2008; Feroz et al., 2009; Feroz et al., 2013), modificado para contemplar los espectros de potencias de los modelos de colapso. 


\section{Capítulo 3}

\section{Inflación}

En este capítulo se describe el modelo cosmológico estándar previo a la introducción de una etapa inflacionaria, así como también algunas de las dificultades que enfrenta. Luego de describir la motivación para introducir una etapa de expansión acelerada en el Universo temprano, se presenta un breve resumen del escenario de inflación estándar. Las secciones están basadas en el libro "Physical Foundations of Cosmology" de V. Mukhanov, los apuntes de D. Baumann "Cosmology, Part III Mathematical Tripos" y el libro "Introduction to Cosmology" de Barbara Ryden.

\subsection{Introducción}

Según el modelo cosmológico estándar el Universo está descripto por la teoría de la Relatividad General de Einstein para un fluido ideal en expansión. Dicha descripción se lleva a cabo utilizando la métrica de Friedmann-Robertson-Walker (FRW), de modo que el elemento de línea se expresa del siguiente modo:

$$
d s^{2}=-d t^{2}+a^{2}(t)\left[\frac{d r^{2}}{1-\kappa r^{2}}+r^{2} d \Omega^{2}\right],
$$

donde $a(t)$ es el factor de expansión, $d \Omega^{2} \equiv d \theta^{2}+\sin ^{2} \theta d \phi^{2}$ siendo $\theta$ y $\phi$ las coordenadas angulares y $\kappa$ da cuenta de la curvatura del espacio:

$$
\kappa \equiv\left\{\begin{array}{cl}
0 & \text { plano (euclídeo) } \\
+1 & \text { esférico (cerrado) } \\
-1 & \text { hiperbólico (abierto) }
\end{array}\right.
$$

La expresión (3.1) no depende de ningún gauge en particular. Reescaleando la coordenada radial de modo que $d \chi \equiv d r \sqrt{1-\kappa r^{2}}$, e introduciendo el tiempo conforme $d \eta=d t / a(t)$, se obtiene:

$$
d s^{2}=a^{2}(\eta)\left[-d \eta^{2}+\left(d \chi^{2}+S_{\kappa}^{2}(\chi) d \Omega^{2}\right)\right],
$$

donde convención de signos utilizada es $(-,+,+,+)$, y

$$
S_{\kappa}(\chi) \equiv\left\{\begin{array}{cl}
\sinh \chi, & \text { si } \kappa=-1 \\
\chi, & \text { si } \kappa=0 \\
\sin \chi, & \text { si } \kappa=+1
\end{array}\right.
$$


La dinámica del Universo estará regida por las ecuaciones de Einstein

$$
G_{\mu \nu}=8 \pi G T_{\mu \nu}=R_{\mu \nu}-\frac{1}{2} R g_{\mu \nu},
$$

donde el tensor de energía-impulso describe un fluido ideal visto desde un observador comóvil. La evolución está determinada también a través de sus componentes, es decir las contribuciones a la densidad y presión que forman parte del tensor energía-impulso. Estas son: materia (materia oscura, bariónica, etc.), radiación y energía oscura. La ecuación de estado para estos componentes de la densidad es $\omega=P / \rho$, donde $P$ es la presión $\rho$ la densidad y $\omega=0$ para la materia oscura y materia bariónica, $\omega=1 / 3$ para la radiación y $\omega=-1$ para la energía oscura. Estas densidades están relacionadas con el factor de escala del siguiente modo (se normaliza a $\left.a\left(t_{\text {hoy }}\right)=1\right)$ :

$$
\rho \propto a^{-3(1+\omega)}
$$

De las ecuaciones de Einstein 00 e $i j$ con $(i=j)$, se obtienen las ecuaciones de Friedmann:

$$
\left(\frac{\dot{a}}{a}\right)^{2}=\frac{8 \pi G}{3} \rho-\frac{k}{a^{2}}, \quad \frac{\ddot{a}}{a}=-\frac{4 \pi G}{3}(\rho+3 P),
$$

donde $\rho$ y $P$ deben ser entendidas como la suma de todas las contribuciones a la densidad de energía y a la presión en el Universo. Definiendo el parámetro de Hubble como $H \equiv \dot{a} / a$, la primera ecuación de Friedmann suele escribirse también como

$$
H^{2}=\frac{8 \pi G}{3} \rho=\frac{8 \pi G}{3}\left(\rho_{r}+\rho_{m}+\rho_{\kappa}+\rho_{\Lambda}\right) .
$$

La densidad crítica al momento presente para un Universo plano $(\kappa=0)$ es

$$
\rho_{\text {crit }, 0}=3 H_{0}^{2} / 8 \pi G,
$$

siendo $H_{0}$ el valor actual del parámetro de Hubble. Es común expresar las ecuaciones de Friedmann en términos del parámetro adimensional $\Omega_{I}=\rho_{I, 0} / \rho_{\text {crit }, o}$, donde $I$ representa cada una de las componentes de la densidad. Teniendo en cuenta que $a_{0} \equiv 1$ se puede escribir:

$$
\frac{H^{2}}{H_{0}^{2}}=\Omega_{r} a^{-4}+\left(\Omega_{B}+\Omega_{C D M}\right) a^{-3}+\Omega_{\kappa} a^{-2}+\Omega_{\Lambda},
$$

donde $\Omega_{r}$ es la densidad de radiación, la densidad total de materia $\Omega_{m}$ está compuesta por la contribución de la materia bariónica $\Omega_{B}$ y la materia oscura $\Omega_{C D M}, \Omega_{\kappa}$ es la densidad correspondiente a la curvatura, y $\Omega_{\Lambda}$ la densidad de energía oscura; cada una expresada en términos de la densidad crítica definida en la expresión (3.7). Los distintos factores de escala indican que, a lo largo de la historia del Universo, fue distinta la predominancia de una u otra componente, según se puede ver en la figura 3.1.

\subsection{El problema del horizonte}

El tamaño de una región del espacio que puede estar en contacto causal queda determinado por cuán lejos puede viajar la luz en determinado intervalo de tiempo. Un fotón viaja a través del espacio tiempo a lo largo de geodésicas nulas $d s^{2}=0$, y eligiendo un sistema de coordenadas tal que su viaje transcurra sobre el eje radial, la ecuación (3.2) se puede expresar:

$$
d \chi= \pm d \eta
$$




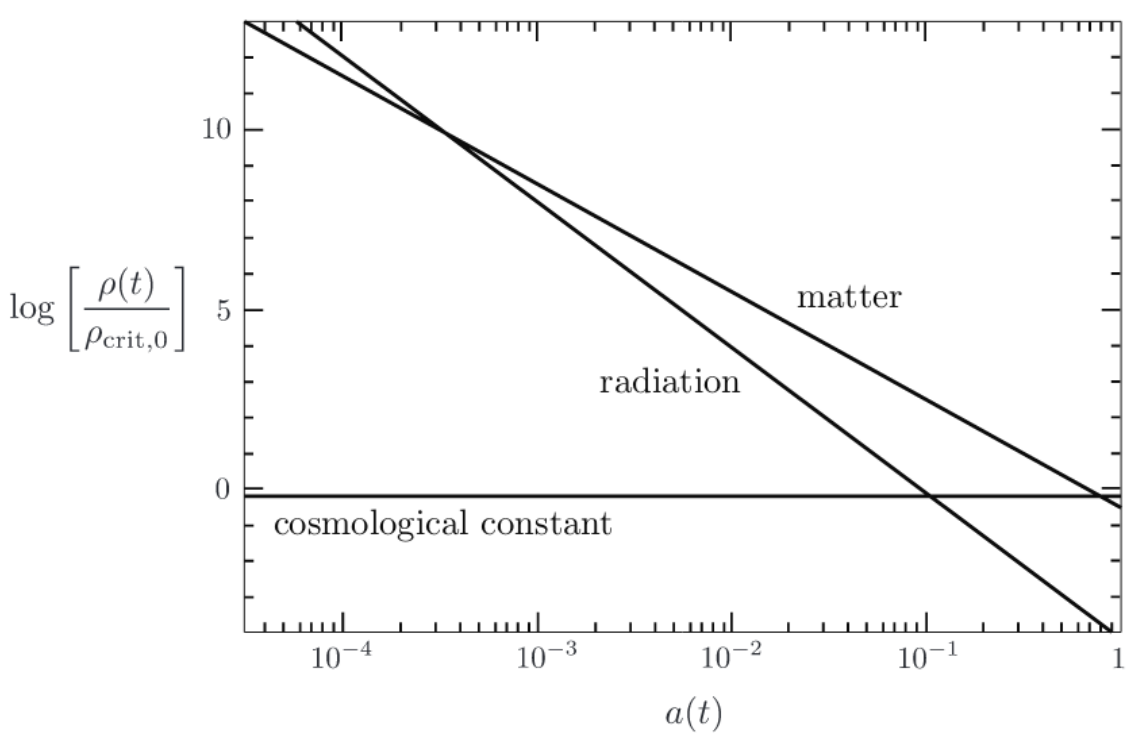

Figura 3.1. Evolución de las densidades de energía en el Universo. Créditos: Baumann, Cosmology Lectures Part III.

definiendo lo que se conoce como el cono de luz. Esto permite definir dos regiones límites u horizontes: uno que limita la distancia a la cual los eventos pasados pueden ser observados, y otro que limita la distancia a la cual un observador en el futuro podrá detectar una señal emitida en el vértice del cono de luz.

Horizonte de partículas La ecuación (3.9) determina la máxima distancia comóvil que la luz puede recorrer entre dos tiempos $\eta_{1}$ y $\eta_{2}$, siendo $\eta_{2}>\eta_{1}$, la cual es simplemente $\eta_{2}-\eta_{1}$ definiendo $c \equiv 1$. Si la singularidad del Big Bang tiene lugar en un instante $t_{i}=0$, entonces la mayor distancia comóvil desde la cual un observador a tiempo $t$ podrá recibir señales que viajen a la velocidad de la luz está dado por

$$
\chi_{h p}(\eta)=\eta-\eta_{i}=\int_{t_{i}}^{t} \frac{d t}{a(t)} .
$$

Esto es lo que se llama el horizonte comóvil de partículas. Su tamaño puede ser visualizado gráficamente en la figura 3.2 como la intersección entre el cono de luz pasado de un observador $p$ y la superficie espacial determinada por $\eta=\eta_{i}$. Todo lo que se encuentra dentro de esta región está conectado causalmente con $p$, y su contorno constituye el horizonte de partículas del observador $p$.

Horizonte de eventos Antes se describió un límite para eventos pasados fuera del cual nunca se recibirá información. También puede definirse una región fuera de la cual las señales emitidas a un tiempo $\eta$ no serán recibidas por un observador en el futuro. La mayor distancia, en coordenadas comóviles, para la cual una señal emitida tiempo $t$ será recibida por un observador en el futuro es:

$$
\chi_{h e}(\eta)=\eta_{f}-\eta=\int_{t}^{t_{f}} \frac{d t}{a(t)} .
$$

Esta región límite se conoce como horizonte comóvil de eventos. 


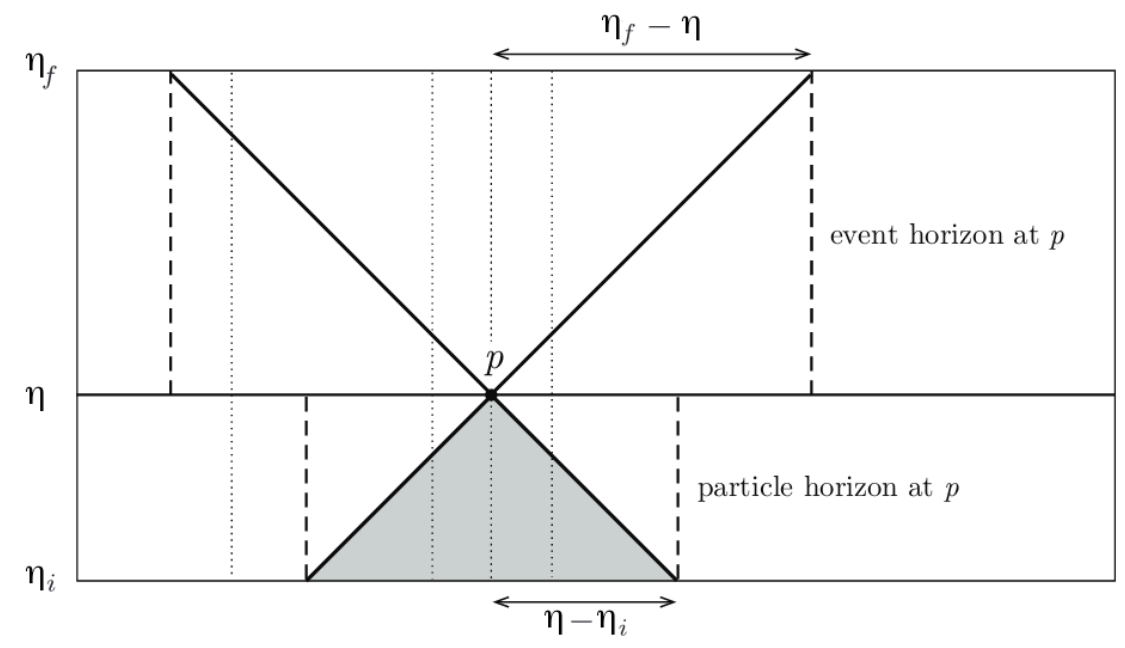

Figura 3.2. Ilustración de los conceptos de horizonte de eventos y horizonte de partículas. Créditos: Baumann, Cosmology Lectures Part III.

Existe además una escala característica de gran importancia que es comúnmente denominada en la literatura como "horizonte comóvil de Hubble", si bien estrictamente no se trata de un horizonte como los descriptos anteriormente.

La ecuación (3.10) puede reescribirse del siguiente modo:

$$
\chi_{h p}(\eta)=\int_{t_{i}}^{t} \frac{d t}{a(t)}=\int_{a_{i}}^{a} \frac{d a}{a \dot{a}}=\int_{\ln a_{i}}^{\ln a}(a H)^{-1} d \ln a,
$$

donde $a_{i}=0$ corresponde a la singularidad del Big Bang. El factor $(a H)^{-1}$ es lo que se denomina radio comóvil de Hubble.

Si la materia satisface la condición fuerte para la energía (strong energy condition) $1+3 \omega>0$, durante la época de dominada por radiación el horizonte de partículas es aproximadamente $1 / a H$, lo cual hace que muchas veces se confundan el radio de Hubble comóvil con el horizonte comóvil de partículas. Durante el período inflacionario el horizonte de partículas no coincide con el radio de Hubble, pero este último sí tiene una noción de causalidad asociada: dos eventos separados por una distancia comóvil mayor que el radio comóvil de Hubble dejarán de estar en contacto causal luego de un tiempo de Hubble. Es común encontrar en la literatura una fusión entre ambas denominaciones a la que se hace referencia como "horizonte de Hubble". Conceptualmente el radio de Hubble es distinto a un horizonte.

En el contexto de inflación, la escala de Hubble se define como $1 / H$ y es una escala característica que denota un radio a partir del cual comienza a ser relevante la curvatura del espacio. En otras palabras, define un sector del espacio-tiempo en que un sistema puede considerarse inercial. La evolución de las escalas características de las perturbaciones en la época de inflación están íntimamente relacionadas a la escala de Hubble. Mientras que el horizonte de partículas está determinado por consideraciones cinemáticas, la escala de Hubble es una medida de la curvatura dinámica que caracteriza la tasa de expansión y describe la evolución de las perturbaciones cosmológicas.

En esta tesis se adoptará la convención utilizada en la literatura y se hará referencia al "horizonte" cuando se aluda al radio comóvil de Hubble.

Como se describió en el capítulo 1, la radiación del FCR es isótropa hasta en una parte en diez mil. La distribución de materia a gran escala presenta un alto grado de homogeneidad 
e isotropía a escalas mayores a unos cientos de megaparsecs. Esta escala que representa la isotropía debe ser tan grande como la escala del horizonte de partículas al momento presente:

$$
c t_{0} \sim 10^{28} \mathrm{~cm} .
$$

donde $t_{0} \sim 10^{17} \mathrm{~s}$ es la edad actual del Universo. Siendo que éste se expande, surge el interrogante de cuáles fueron las condiciones iniciales que evolucionaron en tal grado de isotropía y homogeneidad. Asumiendo que la expansión del Universo no disuelve las inhomogeneidades, se puede estimar el tamaño aproximado de esta región cuando se originó a un dado tiempo $t=t_{i}$ cuyo factor de escala correspondiente es $a_{i}$ :

$$
l_{i} \sim c t_{0} \frac{a_{i}}{a_{0}} .
$$

Comparando esta escala con el tamaño de una región en contacto causal $l_{c}$ al tiempo $t_{i}$ es $l_{c} \sim c t_{i}$, por lo que se tiene que:

$$
\frac{l_{i}}{l_{c}} \sim \frac{t_{0}}{t_{i}} \frac{a_{i}}{a_{0}} .
$$

Una estimación de este cociente teniendo en cuenta que si el Universo está dominado por la radiación $t_{i} \sim t_{p l}$, donde $t_{p l} \sim 10^{-44}$ s es el tiempo de Planck y su temperatura es $T_{p l} \sim 10^{32} \mathrm{~K}$, es:

$$
\frac{a_{i}}{a_{0}} \sim \frac{T_{0}}{T_{p l}} \sim 10^{-32}
$$

por lo que se obtiene:

$$
\frac{l_{i}}{l_{c}} \sim \frac{10^{17}}{10^{-43}} 10^{-32} \sim 10^{28} .
$$

Esto implica que al tiempo inicial de Planck el tamaño del Universo excedía el tamaño de la escala causalmente conectada por 28 órdenes de magnitud, lo cual implica $10^{84}$ regiones desconectadas causalmente cuya diferencia relativa en densidad de energía debe ser menor que $10^{-4}$. No existe un proceso físico que pueda explicar esta precisión en la determinación de las condiciones iniciales en la distribución de la materia sin intercambiar algún tipo de información.

En lo que respecta al FCR, la edad finita del Universo implica que el tiempo transcurrido entre la singularidad del Big Bang y el tiempo conforme del desacople no es suficiente para que los distintos sectores del cielo que se encuentran a una misma temperatura hayan tenido tiempo de transmitirse información alguna. Se estima que sectores del cielo separados por una distancia angular mayor a un grado se encuentran desconectados causalmente. Esto implica una esfera celeste comprendida por cien mil sectores cuyos conos de luz no han tenido tiempo de solaparse (figura 3.3), lo cual hace imposible de explicar el alto grado de isotropía que presenta el FCR. En esto consiste el problema del horizonte.

\subsection{Problema de la planalidad}

Si bien las observaciones actuales del FCR son consistentes con una métrica de FRW plana, en principio la métrica de FRW permite universos planos, esféricos e hiperbólicos (ver sección 3.1). La relación entre la curvatura y la densidad crítica puede escribirse:

$$
1-\Omega(t)=-\frac{\kappa}{a(t)^{2} H(t)^{2}} .
$$




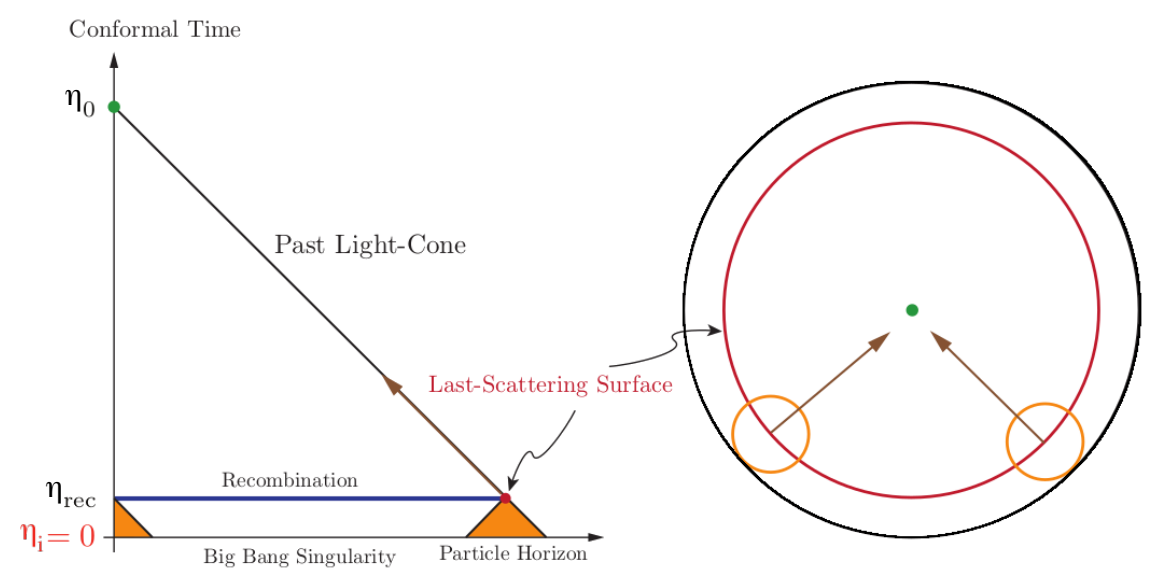

Figura 3.3. Ilustración del problema del horizonte. Teniendo en cuenta la edad del Universo y la superficie de último scattering se tiene que el FCR está compuesto por $10^{5}$ sectores del cielo desconectados causalmente, ya que sus conos de luz pasados no hay tenido suficiente tiempo desde el comienzo del Universo para intersectarse. Créditos: Baumann (2009).

Evaluando esta ecuación al momento presente, se puede dar un límite para este valor consistente con las observaciones de supernovas tipo Ia y el FCR:

$$
\left|1-\Omega_{0}\right| \leq 0.2
$$

La pregunta que surge es por qué la densidad crítica hoy es tan cercana a uno (lo que implica $\kappa \simeq 0$ ), bien podría haber sido órdenes de magnitud mayor o menor sin violar ninguna ley física.

En primera instancia podría suponerse que, sencillamente, las condiciones iniciales del Universo son tales que con su evolución se encuentra $\Omega_{0} \simeq 1$ al tiempo presente. Sin embargo, un análisis simple hace notar que para ello es necesario un ajuste muy fino de la condición inicial. La evolución temporal del parámetro de densidad está dada por

$$
1-\Omega(t)=-\frac{H_{0}^{2}\left(1-\Omega_{0}\right)}{a(t)^{2} H(t)^{2}} .
$$

Para tiempos muy anteriores a la era actual donde el Universo está dominado por la materia y la radiación, se tiene que

$$
\frac{H^{2}}{H_{0}^{2}}=\Omega_{r, 0} a^{-4}+\Omega_{m, 0} a^{-3} .
$$

La combinación de estas dos últimas ecuaciones permite verificar que $\Omega$ se desvía crecientemente de la unidad:

$$
|1-\Omega|_{r} \propto a^{2} \propto t, \quad|1-\Omega|_{m} \propto a \propto t^{2 / 3}
$$

Extrapolando al tiempo de Planck $t_{p l} \approx 5 \times 10^{-44} \mathrm{~s}\left(a_{p l} \approx 2 \times 10^{-32}\right)$, se encuentra que el parámetro de densidad no debió apartarse de la unidad por más de una parte en $10^{60}$, lo cual requiere un ajuste fino de las condiciones iniciales del Universo. Cualquier ínfimo apartamiento hubiera determinado evoluciones drásticamente distintas para el Universo que observamos, si es que siquiera hubiese podido evolucionar hasta tal punto. Esto es lo que se denomina problema de la planalidad. 


\subsection{Expansión acelerada del Universo como solución al pro- blema de los horizontes}

Para un universo dominado por un fluido con una ecuación de estado $\omega \equiv P / \rho$, el radio de Hubble puede expresarse:

$$
(a H)^{-1}=H_{0}^{-1} a^{\frac{1}{2}(1+3 \omega)} .
$$

Si se cumple la condición fuerte para la energía $(1+3 \omega>0)$, el radio comóvil de Hubble crece con la expansión del Universo.

La integral (3.12) está dominada por el límite superior y recibe mínima contribución de los primeros instantes del Universo. Para un fluido perfecto, tomando en cuenta (3.23) se obtiene:

$$
\chi_{h p}(a)=\frac{2 H_{0}^{-1}}{(1+3 \omega)}\left[a^{\frac{1}{2}(1+3 \omega)}-a_{i}^{\frac{1}{2}(1+3 \omega)}\right] \equiv \eta-\eta_{i} .
$$

El hecho de que la mayor contribución al horizonte comóvil está dada por tiempos más tardíos puede hacerse de manifiesto con los siguientes límites:

$$
\eta_{i} \equiv \frac{2 H_{0}^{-1}}{(1+3 \omega)} a_{i}^{\frac{1}{2}(1+3 \omega)} \stackrel{a_{i} \rightarrow 0, \omega>-1 / 3}{\longrightarrow} \quad 0
$$

por lo que el horizonte comóvil es finito.

El radio de Hubble juega un rol fundamental para describir el modelo cosmológico estándar. La propuesta de inflación es incorporar una etapa donde se viola la condición fuerte para la energía y el radio de Hubble decrece para una etapa finita del Universo temprano, implicando además un período de expansión acelerada del Universo. Derivando respecto al tiempo se obtiene:

$$
\frac{d}{d t}(a H)^{-1}=\frac{d}{d t}(\dot{a})^{-1}=-\frac{\ddot{a}}{(\dot{a})^{2}} .
$$

Por lo tanto, si el radio de Hubble decrece entonces $\ddot{a}>0$. Esto trae como consecuencia que la integral (3.12) sea dominada por su límite inferior. Así el inicio del Universo se traslada hacia un tiempo conforme negativo:

$$
\eta_{i}=\frac{2 H_{0}^{-1}}{(1+3 \omega)} a_{i}^{\frac{1}{2}(1+3 \omega)} \stackrel{a_{i} \rightarrow 0, \omega<-1 / 3}{\longrightarrow}-\infty .
$$

Esto implica un intervalo de tiempo conforme más extenso entre la singularidad del Big Bang y el momento del desacople, permitiendo que puntos alejados en la superficie correspondiente al FCR puedan estar en contacto causal (figura 3.4).

\subsection{Duración de inflación}

Introducir un período de expansión acelerada no basta para solucionar de por sí el problema del horizonte, es necesario también que tenga una duración adecuada para lograr su objetivo. En primera medida, el Universo observable actualmente debe quedar dentro del radio comóvil de Hubble al principio de inflación:

$$
\left(a_{0} H_{0}\right)^{-1}<\left(a_{I} H_{I}\right)^{-1}
$$




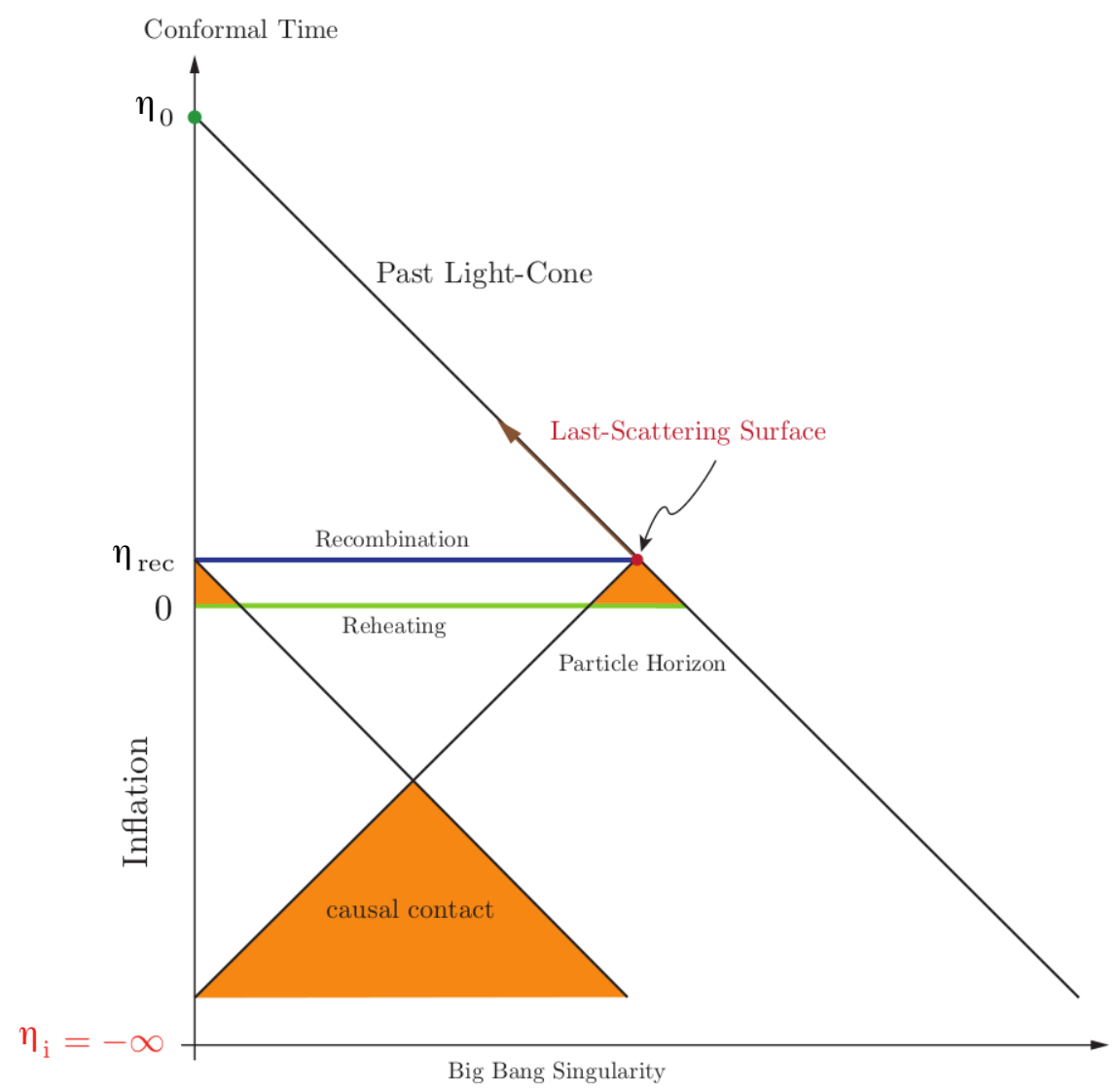

Figura 3.4. La introducción de un período de expansión acelerada proyecta la singularidad inicial hacia tiempos negativos permitiendo que los distintos sectores del cielo hayan tenido tiempo de comunicarse. Créditos: Baumann (2009).

Asumiendo que el Universo estaba dominado por la radiación $\left(H \propto a^{-2}\right)$ una vez finalizado el período inflacionario e ignorando eras más recientes dominadas por la materia y energía oscura se tiene que

$$
\frac{a_{0} H_{0}}{a_{E} H_{E}} \sim \frac{a_{0}}{a_{E}}\left(\frac{a_{E}}{a_{0}}\right)^{2}=\frac{a_{E}}{a_{0}} \sim \frac{T_{0}}{T_{E}} \sim 10^{-28},
$$

donde $T_{0}=10^{-3} \mathrm{eV}(\sim 2.7 \mathrm{~K})$ es la temperatura promedio actual del FCR y $T_{E} \sim 10^{15} \mathrm{GeV}$ es la temperatura al final de inflación, la cual se asume es igual a la energía de la Teoría de Gran Unificación $E_{G U T}$. La ecuación (3.28) se puede expresar:

$$
\left(a_{I} H_{I}\right)^{-1}>\left(a_{0} H_{0}\right)^{-1}=10^{28}\left(a_{E} H_{E}\right)^{-1} .
$$

Para que inflación resuelva el problema del horizonte, $(a H)^{-1}$ debe reducirse por un factor de $10^{28}$. Proponiendo $H \sim$ const durante inflación implica que $H_{I} \approx H_{E}$, y la ecuación (3.30) se puede expresar

$$
\frac{a_{E}}{a_{I}}>10^{28} \Rightarrow \ln \left(\frac{a_{E}}{a_{I}}\right)>64
$$

siendo

$$
N=\int_{a_{I}}^{a_{E}} d \ln a
$$

el número de e-folds. De aquí la afirmación de que se necesitan al menos 60 e-folds de inflación para resolver el problema del horizonte. 
Por otro lado, el aumento del factor de escala entre el principio y el final de inflación es:

$$
\frac{a\left(t_{f}\right)}{a\left(t_{i}\right)}=e^{N}
$$

donde $t_{f}$ y $t_{i}$ indican el tiempo comóvil al final e inicio de inflación respectivamente. Comparando el apartamiento de la unidad para el parámetro de densidad al principio y al final de inflación, se obtiene:

$$
\left|1-\Omega\left(t_{f}\right)\right|=e^{-2 N}\left|1-\Omega\left(t_{i}\right)\right| .
$$

Aunque las condiciones iniciales determinaran valores de $\left|1-\Omega\left(t_{i}\right)\right|$ lejanos a 0 , inflación asegura que el valor final de $\Omega$ será igual a 1 debido a la supresión exponencial con el número de $e$-folds:

$$
\left|1-\Omega\left(t_{f}\right)\right| \sim e^{-2 N} \sim e^{-120} \sim 10^{-53} .
$$

Por esta razón, un período de expansión acelerada cuya duración sea adecuada es también capaz de resolver el problema de la planalidad.

\subsection{La física del período inflacionario}

La etapa inflacionaria del Universo se puede describir mediante la acción de un campo escalar mínimamente acoplado con la gravedad:

$$
S\left[\phi, g_{a b}\right]=\int d^{4} x \sqrt{-g}\left[\frac{1}{16 \pi G} R[g]-\frac{1}{2} \nabla_{a} \phi \nabla_{b} \phi g^{a b}-V[\phi]\right],
$$

siendo $R[g]$ es el escalar de Ricci, $\phi$ el campo escalar inflatón y $V(\phi)$ su potencial.

Variar esta ecuación respecto de la métrica resulta en las ecuaciones $G_{a b}=8 \pi G T_{a b}$. El tratamiento usual es expresar tanto la métrica como el campo inflatón en un fondo isótropo y homogéneo más pequeñas perturbaciones:

$$
\begin{aligned}
g_{a b} & =g_{a b}^{(0)}+\delta g_{a b} \\
\phi & =\phi_{0}(t)+\delta \phi(t, \vec{x}) .
\end{aligned}
$$

El elemento de línea para una métrica de fondo de FRW y perturbaciones escalares, vectoriales y tensoriales se puede expresar en el formalismo invariante de gauge:

$d s^{2}=-(1+2 \varphi) d t^{2}+2 a\left(\partial_{i} B-S_{i}\right) d x^{i} d t+a^{2}\left[(1-2 \psi) \delta_{i j}+\left(2 \partial_{i j} E+\partial_{i} F_{j}+\partial_{j} F_{i}+h_{i j}\right)\right] d x^{i} d x^{j}$

donde

$$
\begin{aligned}
\partial^{i} S_{i} & =0 \\
\partial^{i} F_{i} & =0 \\
h_{i}^{i} & =\partial^{i} h_{i j}=0 .
\end{aligned}
$$

Aquí $\varphi, \psi, E$ y $B$ corresponden a perturbaciones escalares, $S_{i}$ y $F_{i}$ son perturbaciones vectoriales que decaen rápidamente con la expansión del Universo, y por último $h_{i j}$ son las perturbaciones tensoriales. En lo que sigue se analizan las características del fondo sin perturbar, mientras que las perturbaciones se abordarán con detalle en el capítulo 4 . 
El espacio-tiempo de fondo es plano y está caracterizado por una métrica de FRW. Las ecuaciones de Einstein para este caso $G_{00}^{(0)}=8 \pi G T_{00}^{(0)}=8 \pi G a^{2} \rho$ determinan las ecuaciones de Friedmann:

$$
3(\dot{a} / a)^{2}=8 \pi G \rho,
$$

donde las derivadas respecto al tiempo $t$ se indican con un punto (v.g.: $\dot{a}=d a / d t$ ). La expresión para el factor conforme de Hubble $\mathcal{H}=a H$ diferirá si se considera un factor de expansión de tipo de Sitter exacto o cuasi-de Sitter.

El tensor de energía-impulso del campo escalar es

$$
T_{\mu \nu}=\partial_{\mu} \phi \partial_{\nu} \phi-g_{\mu \nu}\left(\frac{1}{2} g^{\alpha \beta} \partial_{\alpha} \phi \partial_{\beta} \phi+V(\phi)\right) .
$$

Dadas las simetrías del espacio-tiempo FRW, el campo sólo puede depender del tiempo: $\phi=\phi(t)$. De la componente temporal $T_{0}^{0}=\rho_{\phi}$ se tiene que la densidad de energía total consta de la suma de dos términos:

$$
\rho_{\phi}=\underbrace{\frac{1}{2} \dot{\phi}^{2}}_{\text {cinético }}+\underbrace{V(\phi)}_{\text {potencial }} .
$$

De las componentes espaciales $T_{j}^{i}=-P_{\phi} \delta_{j}^{i}$ se encuentra que la presión es la diferencia entre la densidad de energía cinética y la densidad de energía potencial:

$$
P_{\phi}=\frac{1}{2} \dot{\phi}^{2}-V(\phi)
$$

Las condiciones para la configuración de una expansión acelerada requieren que $\omega=P / \rho<$ $-1 / 3$, lo cual implica que $P_{\phi}<-\frac{1}{3} \rho_{\phi}$ y el término de energía potencial domina sobre el de energía cinética.

Definiendo la masa reducida de Planck como $M_{p l} \equiv \sqrt{\frac{\hbar c}{8 \pi G}}$, y sustituyendo la ecuación (3.43) en la ecuación (3.6), se obtiene

$$
H^{2}=\frac{1}{3 M_{p l}^{2}}\left[\frac{1}{2} \dot{\phi}^{2}+V(\phi)\right]
$$

Derivando respecto al tiempo y teniendo en cuenta las ecuaciones de Friedmann en (3.41) se desprende

$$
\dot{H}=-\frac{1}{2} \frac{\dot{\phi}^{2}}{M_{p l}^{2}} .
$$

De la combinación de estos dos últimos resultados se obtiene la ecuación de Klein-Gordon:

$$
\ddot{\phi}+3 H \dot{\phi}+\partial_{\phi} V(\phi)=0
$$

que determina la evolución del campo escalar.

\subsection{Inflación slow-roll}

Se denomina condición de slow-roll para inflación cuando la contribución del término cinético a la densidad de energía total es mínima, lo cual se cumple si (ver ecuación (3.46)):

$$
-\frac{\dot{H}}{H^{2}}=\frac{\frac{1}{2} \dot{\phi}^{2}}{M_{p l}^{2} H^{2}} \equiv \epsilon_{H}<1 .
$$


Para que esta condición persista, la aceleración del campo escalar debe ser pequeña. Definiendo una cantidad adimensional de aceleración por tiempo de Hubble

$$
\xi_{H} \equiv-\frac{\ddot{\phi}}{H \dot{\phi}}
$$

y derivando (3.48) respecto al tiempo se obtiene

$$
\delta_{H}=\frac{\dot{\epsilon}_{H}}{H \epsilon_{H}}=2 \frac{\ddot{\phi}}{H \dot{\phi}}-2 \frac{\dot{H}}{H^{2}}=2\left(\epsilon_{H}-\xi_{H}\right) ;
$$

por lo que $\left\{\epsilon_{H},\left|\xi_{H}\right|\right\} \ll 1 \Rightarrow\left\{\epsilon_{H},\left|\delta_{H}\right|\right\} \ll 1$.

Con estas condiciones se puede simplificar la ecuación de movimiento en lo que se conoce como aproximación slow-roll, ya que $\epsilon_{H} \ll 1 \Rightarrow \frac{1}{2} \dot{\phi}^{2} \ll V$, y la ecuación de Friedmann (3.45) se simplifica:

$$
H^{2} \approx \frac{V}{3 M_{p l}^{2}} .
$$

En la aproximación slow-roll la expansión está completamente determinada por la energía potencial. La condición $\xi_{H} \ll 1$ simplifica la ecuación de Klein-Gordon (3.47):

$$
3 H \dot{\phi} \approx-\partial_{\phi} V(\phi) .
$$

La combinación de estas últimas dos ecuaciones brindan la siguiente relación:

$$
\epsilon_{H}=\frac{\frac{1}{2} \dot{\phi}^{2}}{M_{p l}^{2} H^{2}} \approx \frac{M_{p l}^{2}}{2}\left(\frac{\partial_{\phi} V}{V}\right)^{2} \equiv \epsilon_{V}
$$

Derivando (3.52) respecto al tiempo se obtiene

$$
3 \dot{H} \dot{\phi}+3 H \ddot{\phi}=-\dot{\phi} \partial_{\phi \phi} V .
$$

que finalmente resulta

$$
\xi_{H}+\epsilon_{H}=-\frac{\ddot{\phi}}{H \dot{\phi}}-\frac{\dot{H}}{H^{2}} \approx M_{p l}^{2} \frac{\partial_{\phi \phi} V}{V} \equiv \delta_{V} .
$$

La manera de determinar si una región sobre un dado potencial $V(\phi)$ puede dar cuenta de la inflación tipo slow-roll es calcular los parámetros de slow-roll:

$$
\epsilon_{V} \equiv \frac{M_{p l}^{2}}{2}\left(\frac{\partial_{\phi} V}{V}\right)^{2}, \quad\left|\delta_{V}\right| \equiv M_{p l}^{2} \frac{\left|\partial_{\phi \phi} V\right|}{V},
$$

que deben ser $\ll 1$.

Además de estas cantidades, pueden definirse otros parámetros de slow-roll según sea conveniente para describir algún modelo particular. En la sección 4.5 se utilizarán las siguientes definiciones:

$$
\begin{aligned}
\epsilon_{1} & \equiv \epsilon_{V} \\
\epsilon_{2} & \equiv 4 \epsilon_{V}-2 \delta_{V}
\end{aligned}
$$

En esta tesis no se abordará ningún potencial en particular, si no que simplemente se supondrá que el potencial genérico $V(\phi)$ cumple con las correspondientes condiciones de slow-roll. 


\subsection{El problema de la ruptura de simetrías}

En el contexto del modelo cosmológico estándar, el estado inicial del campo inflatón está descripto por un estado de vacío perfectamente isótropo y homogéneo, es decir que resulta invariante ante los operadores cuánticos generadores de translaciones espaciales y rotaciones (Landau et al., 2013; Perez et al., 2006). Además, el espacio-tiempo está caracterizado por la métrica de FRW que es también isótropa y homogénea. La dinámica posterior está regida por la ecuación de Schrödinger y las ecuaciones de Einstein, las cuales respetan estas simetrías. La evolución unitaria de la mecánica cuántica no permite explicar la ruptura de estas simetrías. Por lo tanto, es necesario proveer una explicación detallada del surgimiento de las anisotropías e inhomogeneidades que constituyen las semillas de las estructuras a gran escala que observamos actualmente, partiendo de un estado inicial perfectamente isótropo y homogéneo.

La ruptura de la simetría de un estado cuántico inicial puede darse en el contexto del laboratorio cuando se realiza una medición. En particular, la función de onda asociada al estado cuántico inicial colapsa a un autoestado del observable que se midió. La pérdida de la simetría del estado inicial sucede cuando el operador cuántico asociado al observable medido no conmuta con el operador generador de la simetría original. En cosmología, este es un problema no menor, ya que el origen mismo del Universo no puede ser consecuencia de una medición previo a la existencia misma de cualquier observador o aparato de medición.

Esto se relaciona también con otra problemática ampliamente discutida: la transición del régimen cuántico al régimen clásico. Este tema ha sido abordado en la literatura y se ha propuesto la decoherencia como una se las posibles soluciones (Kiefer \& Joos, 1999). Sin embargo, la decoherencia no puede explicar la ruptura de simetrías.

El Dr. Daniel Sudarsky y colaboradores propusieron un marco conceptual para intentar resolver este problema. La propuesta consiste en incluir en el escenario de inflación estándar el colapso autoinducido de la función de onda del campo inflatón. De esta manera, dicho colapso será el responsable de generar un nuevo estado distinto al del vacío inicial y que, por ende, no comparte sus simetrías. Los detalles se abordarán con detenimiento en el capítulo 4. 


\section{Capítulo 4}

\section{Modelos de colapso}

\subsection{Introducción}

La propuesta del mecanismo del colapso autoinducido del campo inflatón se encuentra actualmente en desarrollo. Para describir la dinámica del mismo se han desarrollado dos propuestas (Cañate et al., 2013; de Unánue \& Sudarsky, 2008; Diez-Tejedor \& Sudarsky, 2012; León et al., 2016, 2011; León \& Sudarsky, 2010; Perez et al., 2006):

- Esquemas de colapso

- Mecanismo CSL, del inglés Continuous Spontaneous Localization.

Ambos tratamientos proponen una transición entre un estado inicial isótropo y homogéneo a un estado que ya no comparte las simetrías iniciales (Sudarsky, 2011). El colapso autoinducido de la función de onda del campo inflatón es entonces el responsable del surgimiento de las anisotropías e inhomogeneidades en cada escala (Diez-Tejedor \& Sudarsky, 2012; León et al., 2011). La diferencia entre ambos mecanismos reside en la dinámica del colapso.

En la propuesta de los esquemas de colapso, el colapso autoinducido del campo inflatón es una hipótesis adicional que se agrega al escenario de inflación estándar (de Unánue \& Sudarsky, 2008; León \& Sudarsky, 2010; Perez et al., 2006). A su vez, cada esquema de colapso consiste en una elección particular de las variables que colapsan (campo y/o momento conjugado) y del estado post-colapso para cada modo $k$ del campo inflatón. El colapso sucede en un momento específico que se denomina tiempo conforme de colapso $\eta_{\vec{k}}^{c}$. Su parametrización no es única, pero sí debe cumplir con ciertas restricciones físicas y observacionales. La forma funcional que adquiera determinará la cantidad de parámetros libres que introduce un esquema específico de colapso y que deben estimarse utilizando datos cosmológicos.

El mecanismo de colapso CSL propone en cambio una modificación de la ecuación de Schrödinger, de modo que el colapso autoinducido de la función de onda del campo inflatón ocurre como parte de la evolución propia del sistema. Este colapso se da de manera continua y espontánea, de modo que no se caracteriza la ocurrencia del colapso mediante la determinación de un tiempo específico. Los enfoques son distintos, ya que en los esquemas de colapso la evolución del estado inicial al estado post-colapso es discreta, mientras que en CSL dicha evolución es continua.

En cuando a la propuesta de los esquemas de colapso y las restricciones impuestas al tiempo conforme de colapso, se han estudiado dos casos: el colapso sucede durante el período 
de inflación (León et al., 2015b) o bien en un tiempo posterior mientras el Universo está dominado por la radiación (León et al., 2014).

Los desarrollos teóricos iniciales se llevaron a cabo en una métrica de fondo de Sitter exacto, obteniendo espectros primordiales de fluctuaciones invariantes de escala, lo cual está descartado con mucha precisión por las observaciones actuales. Por ello, uno de los objetivos de esta tesis fue extender el tratamiento de los modelos de interés al escenario donde el factor de escala fuera de tipo cuasi-de Sitter (León et al., 2015b).

Las siguientes secciones describen el estudio de las distintas propuestas de colapso. Los desarrollos teóricos y las figuras están basados en las publicaciones León et al. (2014, 2015b); Piccirilli et al. (2018), entre otras. El análisis estadístico que contrasta las predicciones teóricas con datos del FCR y BAO, entre otros, se describe en el capítulo 5.

\subsection{La aproximación semiclásica}

Como se mencionó en la última sección del capítulo 3, la propuesta del Dr. Daniel Sudarsky y colaboradores consiste en incluir el colapso autoinducido de la función de onda de los modos del campo inflatón como el mecanismo responsable del surgimiento de las anisotropías e inhomogeneidades. Por otra parte, los resultados obtenidos en el contexto del modelo cosmológico estándar indican que el espectro primordial de fluctuaciones es cuasi-invariante de escala, lo cual equivale a considerar durante inflación una métrica cuasi-de Sitter. En esta tesis se desarrollan las predicciones de varios modelos particulares de colapso con el propósito de poder realizar comparaciones con las cantidades observables. En particular, se calcula el espectro primordial de las fluctuaciones para el caso en que la métrica de fondo es cuasi-de Sitter.

El estado cuántico posterior al colapso ya no tiene porqué ser isótropo ni homogéneo y, por lo tanto, no es autoestado de los operadores de momento lineal y angular. Se puede proponer entonces un mecanismo de colapso y luego calcular los observables en ese nuevo estado.

En el marco del modelo de inflación estándar tanto la métrica como la materia son cuantizadas, introduciendo la variable de Mukhanov-Sasaki. En los modelos de colapso que se describen en esta tesis, se utilizará el enfoque semiclásico de la gravedad, donde solamente la materia será cuantizada a través del campo inflatón. De este modo, la gravedad permanece siempre clásica mientras que la materia es descripta mediante el formalismo de la Teoría Cuántica de Campos en espacio-tiempo curvos.

Como se discutió en la sección anterior, el mecanismo por el cual se produce el colapso no surge naturalmente en el marco del modelo de inflación estándar y hay distintas propuestas que requieren de una hipótesis adicional. Se espera que en el futuro una teoría adecuada de la gravedad cuántica pueda proveer una explicación natural al surgimiento del colapso y su mecanismo. Si bien aún no se ha terminado de desarrollar una teoría completa, existen grandes avances y discusiones en la literatura al respecto (Hooft et al., 2016; Rovelli, 2003). En la ausencia de una teoría de gravedad cuántica, la aproximación semiclásica a la gravedad es una descripción adecuada. Así, la aproximación semiclásica para las ecuaciones de Einstein puede ser utilizada como una teoría efectiva, y en este contexto se elaborarán propuestas fenomenológicas para la dinámica del colapso autoinducido de la función de onda del campo inflatón.

Las ecuaciones semiclásicas de Einstein son aquellas donde la métrica es descripta me- 
diante un formalismo clásico y la materia es descripta por la teoría cuántica de campos en un espacio-tiempo curvo. La métrica y los campos de materia se relacionan mediante:

$$
G_{a b}=8 \pi G\left\langle\hat{T}_{a b}\right\rangle \text {. }
$$

Estas permiten relacionar en el período inflacionario el valor de expectación las perturbaciones cuánticas del campo inflatón con las correspondientes perturbaciones de la métrica (que se mantienen siempre clásicas). Asumir un mecanismo particular de colapso no altera la evolución de la perturbación de la métrica, pero modificará las condiciones iniciales de su ecuación de movimiento. Esta a su vez contiene información acerca del colapso ocurrido en la función de onda del campo inflatón.

La ventaja principal del tratamiento semiclásico es que la descripción tanto de la métrica como de sus perturbaciones permanecen siempre clásicas, mientras que la descripción de la materia es cuántica. De este modo, en el contexto de esta propuesta no aparece el problema de la transición cuántico-clásica.

Un aspecto importante a tener en cuenta es que la aproximación semiclásica no es válida durante el colapso. La introducción de un colapso dinámico viola la conservación de la energía, de modo que la divergencia del tensor energía-momento no se anula: $\nabla_{a}\left\langle\hat{T}^{a b}\right\rangle \neq 0$, lo cual implica a través de las ecuaciones de Einstein que $\nabla_{a} G^{a b} \neq 0$. La divergencia del tensor de Einstein debe ser cero, y por lo tanto las ecuaciones de Einstein semiclásicas pierden validez durante el colapso. Sin embargo, la aproximación semiclásica es válida tanto antes como después del colapso, que son los momentos que resultan de interés para el presente trabajo.

Previo al colapso, el estado inicial del Universo es descripto por el vacío de Bunch-Davies y la métrica de Friedmann-Robertson-Walker, ambos homogéneos e isótropos. Luego algún mecanismo físico (aunque desconocido) induce el colapso a partir del cual el estado cuántico de los campos correspondientes a la materia cambia espontáneamente a un nuevo estado cuántico, que no tiene porqué compartir simetrías con el estado inicial. Por otro lado, los grados de libertad gravitacionales son bien descriptos por las ecuaciones de Einstein luego del colapso. Sin embargo, al evaluar $\left\langle\hat{T}_{a b}\right\rangle$ en el estado post-colapso no necesariamente deberían encontrarse las mismas simetrías que en el estado previo, dando lugar a una geometría que ya no es isótropa ni homogénea.

En este enfoque, la fuente de las perturbaciones en la curvatura tiene su origen en las inhomogeneidades cuánticas del campo inflatón posterior al colapso. Una vez que el colapso tuvo lugar, las inhomogeneidades del inflatón contribuyen a los grados de libertad gravitacionales conduciendo a perturbaciones en las componentes de la métrica, constituyendo una fuente directa de las perturbaciones escalares. Es importante destacar que la métrica en sí misma no constituye una fuente para el colapso autoinducido. Al no actuar el campo escalar como fuente para los modos tensoriales de la métrica, al menos no a primer orden, el análisis concerniente a la amplitud de las ondas gravitacionales primordiales debe ser llevado a segundo orden en las perturbaciones. El cálculo para la amplitud de los modos tensoriales correspondiente a la aproximación semiclásica ha sido realizado por León et al. (2015a); León et al. (2017a,b), encontrándose que la misma está fuertemente suprimida. Por lo tanto, de ser detectados modos de polarización del FCR correspondiente a ondas gravitacionales primordiales, es necesario abandonar la aproximación semiclásica y llevar a cabo la cuantización a través de la variable de Mukhanov-Sasaki. Sin embargo, aún no ha habido detección observacional confirmada de tales modos (BICEP2/Keck Collaboration et al., 2015; Planck Collaboration et al., 2017).

El cálculo del espectro primordial de fluctuaciones en el contexto de la hipótesis del colapso autoinducido utilizando la variable de Mukhanov-Sasaki ha sido realizado por Diez-Tejedor et al. (2012) para los esquemas de colapso y por Bengochea \& León (2017) para CSL. 


\subsection{Antecedentes}

En un trabajo previo se consideraron modelos de colapso con una métrica de fondo deSitter exacto (Landau et al., 2012). En este contexto el factor de Hubble es $H$ es constante $y$, como consecuencia, el espectro primordial se escribe

$$
P(k)=C(k) A_{s},
$$

donde $C(k)$ es una función que condensa la información del desarrollo teórico de los modelos de colapso y su forma varía según el esquema propuesto (de Unánue \& Sudarsky, 2008). La dependencia funcional en $k$ para el modelo cosmológico estándar en el contexto de Sitter exacto implica un espectro primordial de potencias completamente invariante de escala $\left(n_{s}=1\right)^{1}$, lo cual está descartado por las observaciones $\left(n_{s}=0.9655 \pm 0.0062\right.$, Planck Collaboration et al. (2016b)). Sin embargo, los modelos de colapso se sometieron exitosamente a una comparación con los datos ya que las modificaciones introducidas por los esquemas fenomenológicos de colapso derivan en un apartamiento respecto al modelo estándar, a través de la función $C(k)$, que permite reproducir correctamente las observaciones.

En este caso no es posible separar la dependencia en $k$ debido a la dinámica propia del espacio-tiempo de fondo de aquella introducida estrictamente por la propuesta del colapso autoinducido. La importancia de ampliar el desarrollo teórico de los modelos de colapso a un espacio-tiempo de fondo cuasi-de Sitter implica un avance en este sentido, ya que se podrá distinguir más claramente el aporte particular de ambas contribuciones.

Las próximas secciones abordarán con detalle el desarrollo teórico y el análisis de factibilidad de los modelos de colapso autoinducido para el caso de un factor de expansión cuasi-de Sitter, que es justamente uno de los focos principales de esta tesis.

\subsection{Modelos cuasi-de Sitter}

En los modelos inflacionarios de slow roll considerando una expansión cuasi-de Sitter, el factor conforme de Hubble $\mathcal{H} \equiv H / a \equiv a^{\prime}(\eta) / a(\eta)$ está caracterizado a primer orden en los parámetros de slow-roll por $^{2}$

$$
\mathcal{H} \simeq \frac{-1}{\left[\eta\left(1-\epsilon_{H}\right)\right]},
$$

donde las derivadas del factor de escala se toman ahora respecto al tiempo conforme $\eta$ definido como $d \eta=a d t$. De aquí en adelante, las derivadas indicadas con prima (') harán referencia a una diferenciación respecto al tiempo conforme $\eta$. El parámetro de slow roll de Hubble $\epsilon_{H} \equiv 1-\mathcal{H}^{\prime} / \mathcal{H}^{2}$ (definido en la sección 3.7) es $\epsilon_{H} \ll 1$ durante inflación y permanece aproximadamente constante. La ecuación de movimiento para el campo de fondo, teniendo en cuenta la aproximación de slow-roll, puede escribirse $3 \mathcal{H} \phi_{0}^{\prime}=-a^{2} \partial_{\phi} V$.

La normalización del factor de escala se tomará como $a\left(t_{0}\right) \equiv a_{0}=1$ al tiempo cosmológico actual. Teniendo en cuenta una energía para la Teoría de Gran Unificación (Grand Unified Theory) $E_{G U T}=10^{15} \mathrm{GeV}$, el fin de la era inflacionaria tendrá lugar cuando

$$
\eta=\eta_{f} \approx-10^{-22} \mathrm{Mpc},
$$

\footnotetext{
${ }^{1}$ La forma funcional del espectro primordial para el modelo cosmológico estándar es de la forma $P(k)=$ $A_{s} k^{n_{s}-1}$.

${ }^{2}$ A diferencia del caso de Sitter exacto donde el factor conforme de Hubble es $\mathcal{H}=-1 / \eta$.
} 
es decir que el tiempo conforme en el que tiene lugar inflación se sitúa en el intervalo $-\infty<$ $\eta<\eta_{f}$ (ver sección 3.4).

Como se mencionó en la sección 3.6, el procedimiento usual para desarrollar las perturbaciones es descomponer las fluctuaciones de la métrica en términos de sus componentes escalares, vectoriales y tensoriales. Siendo que la amplitud de las perturbaciones vectoriales decae con la expansión del Universo, y además la amplitud de los modos tensoriales en el caso de los modelos semiclásicos se ve fuertemente suprimida (León et al., 2015a), sólo resulta de interés abordar la parte escalar. El elemento de línea se describe en el formalismo invariante de gauge del siguiente modo:

$$
d s^{2}=a^{2}(\eta)\left\{-(1-2 \varphi) d \eta^{2}+2\left(\partial_{i} B\right) d x^{i} d \eta+\left[(1-2 \psi) \delta_{i j}+2 \partial_{i} \partial_{j} E\right] d x^{i} d x^{j}\right\}
$$

en un espacio de fondo caracterizado por la métrica de FRW, donde $\varphi, B, \psi$ y $E$ son las perturbaciones escalares. En esta instancia puede optarse por la elección de un gauge que simplifique la expresión de la métrica o bien trabajar con cantidades invariantes de gauge. Ambos procedimientos arriban a los mismos resultados y son, por ende, equivalentes.

Elegir el gauge longitudinal o Newtoniano permite expresar la métrica del siguiente modo:

$$
d s^{2}=a^{2}(\eta)\left[-(1-2 \varphi) d \eta^{2}+(1-2 \psi) \delta_{i j} d x^{i} d x^{j}\right],
$$

o bien pueden definirse los potenciales de Bardeen que son cantidades invariantes de gauge:

$$
\Phi \equiv \varphi+\frac{1}{a}\left[a\left(B-E^{\prime}\right)\right]^{\prime}, \quad \Psi \equiv \psi+\mathcal{H}\left(E^{\prime}-B\right),
$$

El desarrollo teórico se abordará en esta oportunidad según esta última propuesta utilizando los potenciales de Bardeen.

Las perturbaciones del campo inflatón pueden ser modeladas por fluctuaciones del campo escalar invariante de gauge $\delta \phi^{(\mathrm{GI})}(\eta, \vec{x})=\delta \phi+\phi_{0}^{\prime}\left(B-E^{\prime}\right)$.

Las ecuaciones de Einstein a primer orden en las perturbaciones son $\delta G_{0}^{0}=8 \pi G \delta T_{0}^{0}$, $\delta G_{i}^{0}=8 \pi G \delta T_{i}^{0}$ y $\delta G_{j}^{i}=8 \pi G \delta T_{j}^{i}$, que dan lugar a:

$$
\begin{aligned}
\nabla^{2} \Psi-3 \mathcal{H}\left(\mathcal{H} \Phi+\Psi^{\prime}\right) & =4 \pi G\left[-\phi_{0}^{\prime 2} \Phi+\phi_{0}^{\prime} \delta \phi^{\prime}+\partial_{\phi} V a^{2} \delta \phi\right], \\
\partial_{i}\left(\mathcal{H} \Phi+\Psi^{\prime}\right) & =4 \pi G \partial_{i}\left(\phi_{0}^{\prime} \delta \phi\right), \\
{\left[\Psi^{\prime \prime}+\mathcal{H}(2 \Psi+\Phi)^{\prime}\right.} & \left.+\left(2 \mathcal{H}^{\prime}+\mathcal{H}^{2}\right) \Phi+\frac{1}{2} \nabla^{2}(\Phi-\Psi)\right] \delta_{j}^{i} \\
-\frac{1}{2} \partial^{i} \partial_{j}(\Phi-\Psi) & =4 \pi G\left[\phi_{0}^{\prime} \delta \phi^{\prime}-\phi_{0}^{\prime 2} \Phi-\partial_{\phi} V a^{2} \delta \phi\right] \delta_{j}^{i} .
\end{aligned}
$$

Las perturbaciones de la métrica $\Psi, \Phi$ son cantidades invariantes de gauge (potenciales de Bardeen), mientras que $\delta \phi$ y $\delta \phi^{\prime}$ son las perturbaciones invariantes de gauge asociadas al campo inflatón. Imponer condiciones de contorno apropiadas sobre la última ecuación y considerando $i \neq j$, deriva en la igualdad de los potenciales de Bardeen:

$$
\Psi=\Phi .
$$

De las primeras dos ecuaciones en (4.10) y teniendo en cuenta (3.41), se obtiene la siguiente expresión:

$$
\nabla^{2} \Psi+\mu \Psi=4 \pi G\left(\omega \delta \phi+\phi_{0}^{\prime} \delta \phi^{\prime}\right)
$$

donde $\mu \equiv \mathcal{H}^{2}-\mathcal{H}^{\prime}$ y $\omega \equiv 3 \mathcal{H} \phi_{0}^{\prime}+a^{2} \partial_{\phi} V$. Si se contemplan aproximaciones de slow-roll para la ecuación de movimiento de $\phi_{0}$ se obtiene $3 \mathcal{H} \phi_{0}^{\prime}+a^{2} \partial_{\phi} V \approx 0$, lo cual implica que $\omega \approx 0$. Como consecuencia, la ecuación (4.12) resulta:

$$
\nabla^{2} \Psi+\mu \Psi=4 \pi G \phi_{0}^{\prime} \delta \phi^{\prime} .
$$


En el espacio de Fourier la solución de la ecuación (4.13) se escribe en términos de los parámetros de slow-roll del siguiente modo:

$$
\Psi_{\vec{k}}(\eta)=\sqrt{\frac{\epsilon_{V}}{2}} \frac{H}{M_{P}\left(k^{2}-\mu\right)} a \delta \phi_{\vec{k}}^{\prime}(\eta)^{(\mathrm{GI})},
$$

siendo $H$ el factor de Hubble y $M_{P}^{2} \equiv 1 / 8 \pi G$ la masa de Planck reducida si $\hbar=c=1$.

De la definición de $\mu$ se puede inferir que $\mu=\epsilon_{H} \mathcal{H}^{2}$. Durante la mayor parte del régimen inflacionario se satisface la relación $k^{2} \gg \mu$ (tanto para $|k \eta| \gg 1$ como $|k \eta| \ll 1$ ), lo cual deja de cumplirse hacia el final de inflación cuando $\epsilon_{H}$ se aproxima a la unidad. Sin embargo, como los modos que tienen interés observacional son más grandes que el radio de Hubble comóvil $(|k \eta| \ll 1)$ durante la etapa inflacionaria, la aproximación $k^{2} \gg \mu$ sigue siendo válida y la ecuación (4.14) se puede aproximar por:

$$
\Psi_{\vec{k}}(\eta) \simeq \sqrt{\frac{\epsilon_{H}}{2}} \frac{H}{M_{P} k^{2}} a \delta \phi_{\vec{k}}^{\prime}(\eta)^{(\mathrm{GI})},
$$

que puede ser generalizada a

$$
\Psi_{\vec{k}}(\eta) \simeq \sqrt{\frac{\epsilon_{H}}{2}} \frac{H}{M_{P} k^{2}} a\left\langle\delta \hat{\phi}^{\prime}{ }_{k}(\eta)^{(\mathrm{GI})}\right\rangle .
$$

La ecuación (4.16) ilustra el tratamiento cuántico en los campos de materia (dominados por el inflatón durante inflación), mientras que la perturbación de la métrica permanece siempre clásica.

\subsubsection{Cuantización y caracterización del colapso}

En el esquema semiclásico sólo los campos de materia son cuantizados y el colapso autoinducido es el responsable de generar las perturbaciones de la curvatura. La variable cuántica principal será la fluctuación del campo inflatón $\delta \phi(\vec{x}, \eta)$, que será cuantizada en un espaciotiempo cuasi-de Sitter.

En primera medida se reescalea el campo mediante el cambio de variables $y=a \delta \phi$ y se desarrolla la acción (3.36) a segundo orden en las perturbaciones:

$$
\delta S^{(2)}=\int d^{4} x \frac{1}{2}\left[y^{\prime 2}-(\nabla y)^{2}+\left(\frac{a^{\prime}}{a}\right)^{2} y^{2}-2\left(\frac{a^{\prime}}{a}\right) y y^{\prime}-y^{2} a^{2} \partial_{\phi \phi}^{2} V\right] .
$$

El momento canónico conjugado de $y$ es

$$
\pi \equiv \partial \delta \mathcal{L}^{(2)} / \partial y^{\prime}=y^{\prime}-\left(a^{\prime} / a\right) y=a \delta \phi^{\prime}
$$

Las variables de campo y su momento conjugado son promovidas a operadores, imponiendo las relaciones de conmutación usuales a tiempos iguales:

$$
\begin{aligned}
& {\left[\hat{y}(\vec{x}, \eta), \hat{\pi}\left(\vec{x}^{\prime}, \eta\right)\right]=i \delta\left(\vec{x}-\vec{x}^{\prime}\right),} \\
& {\left[\hat{y}(\vec{x}, \eta), \hat{y}\left(\vec{x}^{\prime}, \eta\right)\right]=\left[\hat{\pi}(\vec{x}, \eta) \hat{\pi}\left(\vec{x}^{\prime}, \eta\right)\right]=0 .}
\end{aligned}
$$

Desarrollando en modos de Fourier, la variables cuánticas campo se pueden expresar:

$$
\begin{aligned}
& \hat{y}(\eta, \vec{x})=\frac{1}{L^{3}} \sum_{\vec{k}} \hat{y}_{\vec{k}}(\eta) e^{i \vec{k} \cdot \vec{x}}, \\
& \hat{\pi}(\eta, \vec{x})=\frac{1}{L^{3}} \sum_{\vec{k}} \hat{\pi}_{\vec{k}}(\eta) e^{i \vec{k} \cdot \vec{x}} .
\end{aligned}
$$


La suma sobre los vectores $\vec{k}$ satisface $k_{i} L=2 \pi n_{i}$ para $i=1,2,3$ con $n_{i}$ entero, dado que la cuantización se toma sobre una caja de longitud $L$. Al final de los cálculos se tomará el límite $L \rightarrow \infty, k \rightarrow$ continuo.

La expresión de la variable de campo y su momento conjugado en términos de los operadores de creación y aniquilación resultan:

$$
\begin{aligned}
& \hat{y}_{\vec{k}}(\eta) \equiv y_{k}(\eta) \hat{a}_{\vec{k}}+y_{k}^{*}(\eta) \hat{a}_{-\vec{k}}^{\dagger}, \\
& \hat{\pi}_{\vec{k}}(\eta) \equiv g_{k}(\eta) \hat{a}_{\vec{k}}+g_{k}^{*}(\eta) \hat{a}_{-\vec{k}}^{\dagger},
\end{aligned}
$$

siendo $g_{k}(\eta)=y_{k}^{\prime}(\eta)-\mathcal{H} y_{k}(\eta)$.

La ecuación de movimiento para $y_{k}(\eta)$ dada por la minimización de la acción (4.17) es

$$
y_{k}^{\prime \prime}(\eta)+\left(k^{2}-\frac{a^{\prime \prime}}{a}+a^{2} \partial_{\phi \phi}^{2} V\right) y_{k}(\eta)=0
$$

De (4.3) se desprende que $a^{\prime \prime} / a \simeq\left(2+3 \epsilon_{H}\right) / \eta^{2}$, que utilizado en conjunto con las ecuaciones de Friedmann y la definición de $\delta_{V}$, se obtiene $a^{2} \partial_{\phi \phi}^{2} V \simeq 3 \delta_{V} / \eta^{2}$; permitiendo reescribir (4.23) del siguiente modo:

$$
y_{k}^{\prime \prime}(\eta)+\left(k^{2}-\frac{2+3\left(\epsilon_{H}-\delta_{V}\right)}{\eta^{2}}\right) y_{k}(\eta)=0,
$$

siendo esta la ecuación de movimiento que habrá que resolver para calcular el espectro de potencias $P(k)$. Los términos con $\epsilon_{H}$ y $\delta_{V}$ surgen de considerar una expansión cuasi-de Sitter.

Las relaciones de conmutación canónica entre $\hat{y}$ y $\hat{\pi}$ establecen que $\left[\hat{a}_{\vec{k}}, \hat{a}_{\vec{k}^{\prime}}^{\dagger}\right]=L^{3} \delta_{\vec{k}, \vec{k}^{\prime}}$, lo cual implica que $y_{k}(\eta)$ debe satisfacer la relación $y_{k} g_{k}^{*}-y_{k}^{*} g_{k}=i$ para todo $k$ en algún tiempo propio $\eta$. La elección de $y_{k}(\eta)$ corresponde a determinar el estado de vacío del campo. En este caso es el vacío de Bunch-Davies:

$$
y_{k}(\eta)=\left(\frac{-\pi \eta}{4}\right)^{1 / 2} e^{i[\nu+1 / 2](\pi / 2)} H_{\nu}^{(1)}(-k \eta)
$$

donde $\nu \equiv 3 / 2+\epsilon_{H}-\delta_{V}$ y $H_{\nu}^{(1)}(-k \eta)$ es la función de Hankel de primera especie de orden $\nu$. ${ }^{3}$ La solución involucra también una fase $e^{i[\nu+1 / 2](\pi / 2)}$, que no será tenida en cuenta ya que carece de consecuencias observacionales.

Para proseguir el análisis cuántico del problema y teniendo en vista que el resultado final debe ser un observable, se separan tanto $\hat{y}_{\vec{k}}(\eta)$ como $\hat{\pi}_{\vec{k}}(\eta)$ en sus partes real e imaginaria:

$$
\begin{aligned}
& \hat{y}_{\vec{k}}(\eta)=\hat{y}_{\vec{k}}^{\mathrm{R}}(\eta)+i \hat{y}_{\vec{k}}^{\mathrm{I}}(\eta), \\
& \hat{\pi}_{\vec{k}}(\eta)=\hat{\pi}_{\vec{k}}^{\mathrm{R}}(\eta)+i \hat{\pi}_{\vec{k}}^{\mathrm{I}}(\eta),
\end{aligned}
$$

de modo que ahora $\hat{y}_{\vec{k}}^{\mathrm{R}, \mathrm{I}}(\eta)$ y $\hat{\pi}_{\vec{k}}^{\mathrm{R}, \mathrm{I}}(\eta)$ son operadores hermíticos. De este modo,

$$
\begin{aligned}
& \hat{y}_{\vec{k}}^{\mathrm{R}, \mathrm{I}}(\eta)=\sqrt{2} \operatorname{Re}\left[y_{k}(\eta) \hat{a}_{\vec{k}}^{\mathrm{R}, \mathrm{I}}\right], \\
& \hat{\pi}_{\vec{k}}^{\mathrm{R}, \mathrm{I}}(\eta)=\sqrt{2} \operatorname{Re}\left[g_{k}(\eta) \hat{a}_{\vec{k}}^{\mathrm{R}, \mathrm{I}}\right],
\end{aligned}
$$

\footnotetext{
${ }^{3}$ Las funciones de Hankel de primera especie están definidas $H_{\nu}^{(1)}(x) \equiv J_{\nu}(x)+i Y_{\nu}(x)$, donde $J_{\nu}$ y $Y_{\nu}$ son las funciones de Bessel de primera y segunda especie respectivamente. Las funciones de Hankel son también conocidas como funciones de Bessel de tercera especie.
} 
donde $\hat{a}_{\vec{k}}^{\mathrm{R}} \equiv\left(\hat{a}_{\vec{k}}+\hat{a}_{-\vec{k}}\right) / \sqrt{2}, \hat{a}_{\vec{k}}^{\mathrm{I}} \equiv-i\left(\hat{a}_{\vec{k}}-\hat{a}_{-\vec{k}}\right) / \sqrt{2}$.

Las relaciones de conmutación para $\hat{a}_{\vec{k}}^{\mathrm{R}, \mathrm{I}}$ no son las usuales, si no que quedan determinadas por

$$
\begin{aligned}
{\left[\hat{a}_{\vec{k}}^{\mathrm{R}}, \hat{a}_{\vec{k}^{\prime}}^{\mathrm{R} \dagger}\right] } & =L^{3}\left(\delta_{\vec{k}, \vec{k}^{\prime}}+\delta_{\vec{k},-\vec{k}^{\prime}}\right), \\
{[\hat{a}} & \left.\mathrm{I}, \hat{a}_{\vec{k}^{\prime}}^{\mathrm{I}}\right]=L^{3}\left(\delta_{\vec{k}, \vec{k}^{\prime}}-\delta_{\vec{k},-\vec{k}^{\prime}}\right),
\end{aligned}
$$

siendo el resto nulas.

Antes de proseguir, no está demás enfatizar que el estado de vacío definido por $\hat{a}_{\vec{k}}^{\mathrm{R}, \mathrm{I}}|0\rangle=$ 0 es traslacional y rotacionalmente invariante. La prueba formal puede encontrarse en los apéndices de trabajo de Landau, León, \& Sudarsky (2013).

En lo que sigue se busca formalizar la conexión entre las perturbaciones cuánticas del inflatón con la perturbación primordial de la curvatura. La ecuación (4.16) en términos del valor de expectación del momento conjugado en el gauge longitudinal es

$$
\Psi_{\vec{k}}(\eta) \simeq \sqrt{\frac{\epsilon_{H}}{2}} \frac{H}{M_{P} k^{2}}\left\langle\hat{\pi}_{\vec{k}}(\eta)\right\rangle
$$

En el estado de vacío $\left\langle\hat{\pi}_{\vec{k}}(\eta)\right\rangle_{0}=0$, lo cual implica que $\Psi_{\vec{k}}=0$ : no hay perturbaciones en el espacio-tiempo de fondo. Únicamente después de que el colapso haya tenido lugar es que $\left\langle\hat{\pi}_{\vec{k}}(\eta)\right\rangle_{\Theta} \neq 0$ y entonces $\Psi_{\vec{k}} \neq 0$. Las inhomogeneidades y anisotropías primordiales surgen del colapso cuántico.

El proceso de colapso implica la transición de un estado de vacío completamente isótropo y homogéneo a un nuevo estado $|\Theta\rangle \neq|0\rangle$, donde todos los modos $\vec{k}$ del inflatón son afectados por el colapso. De modo que el estado de vacío original $|0\rangle$ evoluciona en un estado postcolapso:

$$
|\Theta\rangle=\ldots\left|\Theta_{-\mathbf{k}_{2}}\right\rangle \otimes\left|\Theta_{-\mathbf{k}_{1}}\right\rangle \otimes\left|\Theta_{\mathbf{k}_{0}}\right\rangle \otimes\left|\Theta_{\mathbf{k}_{1}}\right\rangle \otimes\left|\Theta_{\mathbf{k}_{2}}\right\rangle \ldots
$$

Desde este nuevo estado posterior al colapso y teniendo en cuenta la ecuación (4.29), puede verse como el valor esperado de $\left\langle\hat{\pi}_{\vec{k}}(\eta)\right\rangle$ actúa como fuente para $\Psi_{\vec{k}}$ para cada $\mathbf{k}$. Estos modos pueden dividirse en dos grandes grupos:

1. $k \ll \mathcal{H}\left(\eta_{\vec{k}}^{c}\right)$ : modos cuya longitud de onda comóvil asociada es mayor que el radio de Hubble comóvil al momento del colapso, caso al que se referirá como fuera del horizonte,

2. $k \gg \mathcal{H}\left(\eta_{\vec{k}}^{c}\right)$ : modos cuya longitud de onda comóvil asociada es menor que el radio de Hubble comóvil al momento del colapso, situación que será denominada dentro del horizonte.

El cálculo prosigue considerando una analogía entre el colapso y una medición imprecisa de los operadores $\hat{y}_{\vec{k}}^{\mathrm{R}, \mathrm{I}}(\eta)$ y $\hat{\pi}_{\vec{k}}^{\mathrm{R}, \mathrm{I}}(\eta)$, donde no se pretende obtener un autoestado del observable si no una función gaussiana centrada en el valor correspondiente al autoestado. Es decir que se necesita especificar la dinámica de los valores de expectación $\left\langle\hat{y}_{\vec{k}}^{\mathrm{R}, \mathrm{I}}(\eta)\right\rangle$ y $\left\langle\hat{\pi}_{\vec{k}}^{\mathrm{R}, \mathrm{I}}(\eta)\right\rangle$ evaluados en el estado posterior al colapso. Para el caso de los esquemas de colapso, la expresión analítica de estos estados va a depender también de los valores de expectación evaluados al tiempo de colapso $\eta_{\vec{k}}^{c}$. 


\subsubsection{Esquemas de colapso}

Se proponen distintos esquemas donde el colapso de la función del onda del campo inflatón se describe de manera fenomenológica, permitiendo así caracterizar los valores de expectación del campo y su momento conjugado evaluados en un estado post-colapso (de Unánue \& Sudarsky, 2008). Se asume que el efecto del colapso autoinducido es análogo al producido por una medición, donde los valores de expectación de los operadores de campo y momento en cada modo serán relacionados con las incertezas del estado inicial. En el estado de vacío tanto $\hat{y}_{\vec{k}}$ como $\hat{\pi}_{\vec{k}}$ se distribuyen conforme a una función gaussiana centrada en cero y con dispersión $\left(\Delta \hat{y}_{\vec{k}}\right)_{0}^{2}$ y $\left(\Delta \hat{\pi}_{\vec{k}}\right)_{0}^{2}$ respectivamente (incerteza previa al colapso). Cada elección particular de las variables de colapso y la expresión para el estado post-colapso se denomina esquema de colapso.

\section{Esquema Independiente}

En este esquema, tanto los valores de expectación de los modos del campo $\hat{y}_{\vec{k}}^{\mathrm{R}, \mathrm{I}}$ como de su momento conjugado $\hat{\pi}_{\vec{k}}^{\mathrm{R}, \mathrm{I}}$ se modifican luego del colapso de la siguiente manera:

$$
\begin{aligned}
\left\langle\hat{y}_{\vec{k}}^{\mathrm{R}, \mathrm{I}}\left(\eta_{\vec{k}}^{c}\right)\right\rangle & =x_{\vec{k}, 1}^{\mathrm{R}, \mathrm{I}} \sqrt{\left(\Delta \hat{y}_{\vec{k}}^{\mathrm{R}, \mathrm{I}}\left(\eta_{\vec{k}}^{c}\right)\right)_{0}^{2}}, \\
\left\langle\hat{\pi}_{\vec{k}}^{\mathrm{R}, \mathrm{I}}\left(\eta_{\vec{k}}^{c}\right)\right\rangle & =x_{\vec{k}, 2}^{\mathrm{R}, \mathrm{I}} \sqrt{\left(\Delta \hat{\pi}_{\vec{k}}^{\mathrm{R}, \mathrm{I}}\left(\eta_{\vec{k}}^{c}\right)\right)_{0}^{2}},
\end{aligned}
$$

donde $x_{\vec{k}, 1}^{(R, I)}$ y $x_{\vec{k}, 2}^{(R, I)}$ son variables aleatorias seleccionadas de una distribución gaussiana centrada en cero y con dispersión unitaria. El valor de expectación del estado post-colapso es, a su vez, proporcional a la incerteza del estado antes del colapso. Las variables no se encuentran correlacionadas, de ahí el nombre del esquema.

Tanto las expresiones para $y_{k}(\eta)$ (ecuación (4.22)) como para $g_{k}(\eta)$ (definido como $g_{k}(\eta)=$ $\left.y_{k}^{\prime}(\eta)-\mathcal{H} y_{k}(\eta)\right)$ pueden descomponerse en su parte real e imaginaria:

$$
\begin{aligned}
& y_{k}(\eta)=\operatorname{Re}\left[y_{k}(\eta)\right]+i \operatorname{Im}\left[y_{k}(\eta)\right], \\
& g_{k}(\eta)=\operatorname{Re}\left[g_{k}(\eta)\right]+i \operatorname{Im}\left[g_{k}(\eta)\right]
\end{aligned}
$$

resultando:

$$
\begin{aligned}
& \operatorname{Re}\left[y_{k}(\eta)\right]=\left(\frac{\pi}{4}\right)^{1 / 2} \frac{\sqrt{-k \eta}}{k^{1 / 2}} J_{\nu}(-k \eta), \\
& \operatorname{Im}\left[y_{k}(\eta)\right]=\left(\frac{\pi}{4}\right)^{1 / 2} \frac{\sqrt{-k \eta}}{k^{1 / 2}} Y_{\nu}(-k \eta), \\
& \operatorname{Re}\left[g_{k}(\eta)\right]=k^{1 / 2}\left(\frac{\pi}{4}\right)^{1 / 2}\left(\frac{-\alpha J_{\nu}(-k \eta)}{\sqrt{-k \eta}}+\sqrt{-k \eta} J_{\nu+1}(-k \eta)\right), \\
& \operatorname{Im}\left[g_{k}(\eta)\right]=k^{1 / 2}\left(\frac{\pi}{4}\right)^{1 / 2}\left(\frac{-\alpha Y_{\nu}(-k \eta)}{\sqrt{-k \eta}}+\sqrt{-k \eta} Y_{\nu+1}(-k \eta)\right) .
\end{aligned}
$$

El factor $\alpha$ es función del orden de las ecuaciones de Bessel y del parámetro de slow-roll:

$$
\alpha \equiv 1 / 2+\nu+1 /\left(1-\epsilon_{H}\right)
$$


De la ecuación (4.27) se puede obtener la forma de las incertezas cuánticas:

$$
\begin{aligned}
\left(\Delta \hat{y}_{\vec{k}}^{\mathrm{R}, \mathrm{I}}\left(\eta_{\vec{k}}^{c}\right)\right)_{0}^{2} & =\frac{L^{3}}{4}\left|y_{k}\left(\eta_{\vec{k}}^{c}\right)\right|^{2} \\
& =\frac{L^{3} \pi\left|z_{k}\right|}{16 k}\left[J_{\nu}^{2}\left(\left|z_{k}\right|\right)+Y_{\nu}^{2}\left(\left|z_{k}\right|\right)\right] \\
\left(\Delta \hat{\pi}_{\vec{k}}^{\mathrm{R}, \mathrm{I}}\left(\eta_{\vec{k}}^{c}\right)\right)_{0}^{2} & =\frac{L^{3}}{4}\left|g_{k}\left(\eta_{\vec{k}}^{c}\right)\right|^{2}=\frac{L^{3} \pi k}{16} \times\left[\left(\frac{-\alpha J_{\nu}\left(\left|z_{k}\right|\right)}{\sqrt{\left|z_{k}\right|}}+\sqrt{\left|z_{k}\right|} J_{\nu+1}\left(\left|z_{k}\right|\right)\right)^{2}\right. \\
& \left.+\left(\frac{-\alpha Y_{\nu}\left(\left|z_{k}\right|\right)}{\sqrt{\left|z_{k}\right|}}+\sqrt{\left|z_{k}\right|} Y_{\nu+1}\left(\left|z_{k}\right|\right)\right)^{2}\right]
\end{aligned}
$$

donde $z_{k} \equiv k \eta_{\vec{k}}^{c}$, siendo $\eta_{\vec{k}}^{c}$ el tiempo propio de colapso para cada modo $k$.

\section{Esquema Newtoniano}

En el esquema de Newton, sólo el valor de expectación para el momento conjugado $\hat{\pi}_{\vec{k}}$ se modifica como consecuencia del colapso autoinducido. Por lo tanto:

$$
\begin{aligned}
\left\langle\hat{y}_{\vec{k}}^{\mathrm{R}, \mathrm{I}}\left(\eta_{\vec{k}}^{c}\right)\right\rangle & =0, \\
\left\langle\hat{\pi}_{\vec{k}}^{\mathrm{R}, \mathrm{I}}\left(\eta_{\vec{k}}^{c}\right)\right\rangle & =x_{\vec{k}, 2}^{\mathrm{R}, \mathrm{I}} \sqrt{\left(\Delta \hat{\pi}_{\vec{k}}^{\mathrm{R}, \mathrm{I}}\left(\eta_{\vec{k}}^{c}\right)\right)_{0}^{2}} .
\end{aligned}
$$

Al igual que en el esquema anterior, $x_{\vec{k}, 2}^{(\mathrm{R}, \mathrm{I})}$ son variables aleatorias seleccionadas de una distribución gaussiana normalizada a 1 y centrada en cero.

La caracterización de la incerteza $\left(\Delta \hat{\pi}_{\vec{k}}^{\mathrm{R}, \mathrm{I}}\left(\eta_{\vec{k}}^{c}\right)\right)_{0}^{2}$ es la misma que para el esquema Independiente, y viene dada por la ecuación (4.40).

\section{Esquema Wigner}

En este esquema, al igual que en el esquema Independiente, tanto la variable del campo como su momento conjugado se modifican con el colapso. A su vez y a diferencia del esquema Independiente, los valores de expectación del estado post-colapso están correlacionados por la función de Wigner ${ }^{4}$ :

$$
\begin{aligned}
& \left\langle\hat{y}_{\vec{k}}^{\mathrm{R}, \mathrm{I}}\left(\eta_{\vec{k}}^{c}\right)\right\rangle=x_{\vec{k}}^{\mathrm{R}, \mathrm{I}} \Lambda_{k}\left(\eta_{\vec{k}}^{c}\right) \cos \Theta_{k}\left(\eta_{\vec{k}}^{c}\right), \\
& \left\langle\hat{\pi}_{\vec{k}}^{\mathrm{R}, \mathrm{I}}\left(\eta_{\vec{k}}^{c}\right)\right\rangle=x_{\vec{k}}^{\mathrm{R}, \mathrm{I}} k \Lambda_{k}\left(\eta_{\vec{k}}^{c}\right) \sin \Theta_{k}\left(\eta_{\vec{k}}^{c}\right),
\end{aligned}
$$

donde $x_{\vec{k}}^{\mathrm{R}, \mathrm{I}}$ son variables aleatorias caracterizadas por una distribución de probabilidad gaussiana centrada en cero y normalizada. El parámetro $\Lambda_{k}\left(\eta_{\vec{k}}^{c}\right)$ representa el semieje mayor de la elipse que caracteriza la gaussiana bidimensional de la función de Wigner. Esta elipse corresponde al contorno de una región en el espacio de fases donde la función de Wigner tiene una magnitud equivalente a la mitad de su valor máximo. Por otro lado, $\Theta_{k}\left(\eta_{\vec{k}}^{c}\right)$ es el ángulo entre ese eje y aquel que determinan $\hat{y}_{\vec{k}}^{\mathrm{R}}, \mathrm{I}$. Las fórmulas explícitas en términos de $z_{k} \equiv k \eta_{\vec{k}}^{c}$,

\footnotetext{
${ }^{4}$ La función de Wigner del estado de vacío es una función gaussiana bidimensional.
} 
siendo $\eta_{\vec{k}}^{c}$ el tiempo conforme de colapso, son:

$$
\begin{aligned}
\Lambda_{k}=(2 L)^{3 / 2} \sqrt{\frac{\pi\left|z_{k}\right|}{4 k}}\left[J_{\nu}^{2}\left(\left|z_{k}\right|\right)+Y_{\nu}^{2}\left(\left|z_{k}\right|\right)\right]^{1 / 2}\left[S\left(\left|z_{k}\right|\right)\right. \\
\left.-\sqrt{S^{2}\left(\left|z_{k}\right|\right)-\left(\frac{\pi\left|z_{k}\right|}{2}\right)^{2}\left(J_{\nu}^{2}\left(\left|z_{k}\right|\right)+Y_{\nu}^{2}\left(\left|z_{k}\right|\right)\right)^{2}}\right]^{-1 / 2}, \\
\tan 2 \Theta_{k}=-\frac{\pi^{2}\left|z_{k}\right|}{4}\left[J_{\nu}^{2}\left(\left|z_{k}\right|\right)+Y_{\nu}^{2}\left(\left|z_{k}\right|\right)\right] \\
\times\left[S\left(\left|z_{k}\right|\right)-\frac{\pi\left|z_{k}\right|}{8}\left(J_{\nu}^{2}\left(\left|z_{k}\right|\right)+Y_{\nu}^{2}\left(\left|z_{k}\right|\right)\right)^{2}\right]^{-1} \\
\times\left[-2 \nu\left(J_{\nu}^{2}\left(\left|z_{k}\right|\right)+Y_{\nu}^{2}\left(\mid z_{k}\right)\right)+\left|z_{k}\right|\right. \\
\left.\times\left(J_{\nu}\left(\left|z_{k}\right|\right) J_{\nu+1}\left(\left|z_{k}\right|\right)+Y_{\nu}\left(\left|z_{k}\right|\right) Y_{\nu+1}\left(\left|z_{k}\right|\right)\right)\right],
\end{aligned}
$$

donde

$$
\begin{aligned}
S\left(\left|z_{k}\right|\right) & \equiv 1+\frac{\pi^{2}}{16}\left\{\left|z_{k}\right|^{2}\left(J_{\nu}^{2}\left(\left|z_{k}\right|\right)+Y_{\nu}^{2}\left(\left|z_{k}\right|\right)\right)^{2}\right. \\
& +4\left[J_{\nu}^{2}\left(\left|z_{k}\right|\right)+Y_{\nu}^{2}\left(\left|z_{k}\right|\right)-\left|z_{k}\right|\left(J_{\nu}\left(\left|z_{k}\right|\right) J_{\nu+1}\left(\left|z_{k}\right|\right)\right.\right. \\
& \left.\left.\left.+Y_{\nu}\left(\left|z_{k}\right|\right) Y_{\nu+1}\left(\left|z_{k}\right|\right)\right)\right]^{2}\right\} .
\end{aligned}
$$

\subsubsection{Perturbaciones en la curvatura}

En lo que sigue se describirán las perturbaciones de la curvatura en términos de los parámetros que caracterizan el colapso. La ecuación (4.29) indica que la evolución de los valores esperados de las perturbaciones de la métrica dependerá sólo del valor de expectación del momento conjugado $\left\langle\hat{\pi}_{\vec{k}}(\eta)\right\rangle$.

Se define la cantidad $d_{\vec{k}}^{\mathrm{R}, \mathrm{I}} \equiv\left\langle\Theta\left|\hat{a}_{\vec{k}}^{\mathrm{R}, \mathrm{I}}\right| \Theta\right\rangle$, que determina el valor de expectación de los operadores del campo y momento conjugado del modo $\vec{k}$ en el estado post-colapso $|\Theta\rangle$. Partiendo de la ecuación (4.27) se obtiene :

$$
\left\langle\hat{\pi}_{\vec{k}}^{\mathrm{R}, \mathrm{I}}(\eta)\right\rangle_{\Theta}=\sqrt{2} \operatorname{Re}\left[g_{k}(\eta) d_{\vec{k}}^{\mathrm{R}, \mathrm{I}}\right] .
$$

A su vez es posible relacionar $d_{\vec{k}}^{R, I}$ con el valor de expectación de los operadores del campo en el momento del colapso:

$$
\begin{aligned}
\left\langle\hat{y}_{\vec{k}}^{\mathrm{R}, \mathrm{I}}\left(\eta_{\vec{k}}^{c}\right)\right\rangle_{\Theta} & =\sqrt{2} \operatorname{Re}\left[y_{k}\left(\eta_{\vec{k}}^{c}\right) d_{\vec{k}}^{\mathrm{R}, \mathrm{I}}\right], \\
\left\langle\hat{\pi}_{\vec{k}}^{\mathrm{R}, \mathrm{I}}\left(\eta_{\vec{k}}^{c}\right)\right\rangle_{\Theta} & =\sqrt{2} \operatorname{Re}\left[g_{k}\left(\eta_{\vec{k}}^{c}\right) d_{\vec{k}}^{\mathrm{R}, \mathrm{I}}\right] .
\end{aligned}
$$

Entonces, utilizando la definición de $d_{\vec{k}}^{\mathrm{R}, \mathrm{I}}$ en términos de los valores de expectación al tiempo de colapso y reemplazando en la ecuación (4.48), se obtiene la expresión para el valor de expectación del operador del momento del campo en términos del valor de expectación al momento del colapso:

$$
\left\langle\hat{\pi}_{\vec{k}}^{\mathrm{R}, \mathrm{I}}(\eta)\right\rangle_{\Theta}=F\left(k \eta, z_{k}\right)\left\langle\hat{y}_{\vec{k}}^{\mathrm{R}, \mathrm{I}}\left(\eta_{\vec{k}}^{c}\right)\right\rangle_{\Theta}+G\left(k \eta, z_{k}\right)\left\langle\hat{\pi}_{\vec{k}}^{\mathrm{R}, \mathrm{I}}\left(\eta_{\vec{k}}^{c}\right)\right\rangle_{\Theta},
$$


donde

$$
\begin{aligned}
F\left(k \eta, z_{k}\right) \equiv & \frac{k \pi}{4}\left\{\left(\frac{-\alpha Y_{\nu}\left(\left|z_{k}\right|\right)}{\sqrt{\left|z_{k}\right|}}+\sqrt{\left|z_{k}\right|} Y_{\nu+1}\left(\left|z_{k}\right|\right)\right)\right. \\
& \times\left(\frac{-\alpha J_{\nu}(|k \eta|)}{\sqrt{|k \eta|}}+\sqrt{|k \eta|} J_{\nu+1}(|k \eta|)\right) \\
& -\left(\frac{-\alpha J_{\nu}\left(\left|z_{k}\right|\right)}{\sqrt{\left|z_{k}\right|}}+\sqrt{\left|z_{k}\right|} J_{\nu+1}\left(\left|z_{k}\right|\right)\right) \\
& \left.\times\left(\frac{-\alpha Y_{\nu}(|k \eta|)}{\sqrt{|k \eta|}}+\sqrt{|k \eta|} Y_{\nu+1}(|k \eta|)\right)\right\}, \\
G\left(k \eta, z_{k}\right) \equiv & \frac{\pi \sqrt{\left|z_{k}\right|}\left\{J_{\nu}\left(\left|z_{k}\right|\right)\right.}{4} \\
\times & {\left[\frac{-\alpha Y_{\nu}(|k \eta|)}{\left.\sqrt{|k \eta|}+\sqrt{|k \eta|} Y_{\nu+1}(|k \eta|)\right]}\right.} \\
- & \left.Y_{\nu}\left(\left|z_{k}\right|\right)\left[\frac{-\alpha J_{\nu}(|k \eta|)}{\sqrt{|k \eta|}}+\sqrt{|k \eta|} J_{\nu+1}(|k \eta|)\right]\right\} .
\end{aligned}
$$

Recordando que la cantidad $z_{k}$ se define como $z_{k} \equiv k \eta_{\vec{k}}^{c}$, siendo $\eta_{\vec{k}}^{c}$ es el tiempo conforme en el cual se produce el colapso del modo $k$.

Sustituyendo la ecuación (4.51) en la ecuación (4.29) se obtiene una expresión para la perturbación de la curvatura en el gauge longitudinal:

$$
\begin{aligned}
\Psi_{\vec{k}}(\eta)= & \sqrt{\frac{\epsilon_{V}}{2}} \frac{H}{M_{P} k^{2}} \times\left[F\left(k \eta, z_{k}\right)\left(\left\langle\hat{y}_{\vec{k}}^{\mathrm{R}}\left(\eta_{\vec{k}}^{c}\right)\right\rangle_{\Theta}+i\left\langle\hat{y}_{\vec{k}}^{\mathrm{I}}\left(\eta_{\vec{k}}^{c}\right)\right\rangle_{\Theta}\right)\right. \\
& \left.+G\left(k \eta, z_{k}\right)\left(\left\langle\hat{\pi}_{\vec{k}}^{\mathrm{R}}\left(\eta_{\vec{k}}^{c}\right)\right\rangle_{\Theta}+i\left\langle\hat{\pi}_{\vec{k}}^{\mathrm{I}}\left(\eta_{\vec{k}}^{c}\right)\right\rangle_{\Theta}\right)\right] .
\end{aligned}
$$

Se puede ver cómo la curvatura depende de los distintos esquemas de colapso a través de $\left\langle\hat{y}_{\vec{k}}^{\mathrm{I}}\left(\eta_{\vec{k}}^{c}\right)\right\rangle_{\Theta} \mathrm{y}\left\langle\hat{\pi}_{\vec{k}}^{\mathrm{I}}\left(\eta_{\vec{k}}^{c}\right)\right\rangle_{\Theta}$, por lo que se obtienen tres expresiones distintas para $\Psi_{\vec{k}}(\eta)$ en función de cada uno de los esquemas propuestos.

Definiendo $X_{\vec{k}} \equiv x_{\vec{k}}^{\mathrm{R}}+i x_{\vec{k}}^{\mathrm{I}}$, la perturbación en la curvatura en el gauge longitudinal para cada uno de los esquemas de colapso toma la siguiente forma:

\section{Esquema Independiente}

$$
\begin{aligned}
\Psi_{\vec{k}}^{\text {ind }}(\eta) & =\frac{\sqrt{L^{3} \pi \epsilon_{V}} H}{2^{5 / 2} M_{P} k^{2}} \\
& \times\left\{F\left(k \eta, z_{k}\right) X_{\vec{k}, 1} \sqrt{\frac{\left|z_{k}\right|}{k}}\left[J_{\nu}^{2}\left(\left|z_{k}\right|\right)+Y_{\nu}^{2}\left(\left|z_{k}\right|\right)\right]^{1 / 2}\right. \\
& +G\left(k \eta, z_{k}\right) X_{\vec{k}, 2} \sqrt{k} \\
& \times\left[\left(\frac{-\alpha J_{\nu}\left(\left|z_{k}\right|\right)}{\sqrt{\left|z_{k}\right|}}+\sqrt{\left|z_{k}\right|} J_{\nu+1}\left(\left|z_{k}\right|\right)\right)^{2}\right. \\
& \left.\left.+\left(\frac{-\alpha Y_{\nu}\left(\left|z_{k}\right|\right)}{\sqrt{\left|z_{k}\right|}}+\sqrt{\left|z_{k}\right|} Y_{\nu+1}\left(\left|z_{k}\right|\right)\right)^{2}\right]^{1 / 2}\right\} .
\end{aligned}
$$




\section{Esquema Newtoniano}

$$
\begin{aligned}
\Psi_{\vec{k}}^{\text {newt }}(\eta) & =\frac{\sqrt{L^{3} \pi \epsilon_{V}} H}{2^{5 / 2} M_{P} k^{2}} G\left(k \eta, z_{k}\right) X_{\vec{k}, 2} \sqrt{k} \\
& \times\left[\left(\frac{-\alpha J_{\nu}\left(\left|z_{k}\right|\right)}{\sqrt{\left|z_{k}\right|}}+\sqrt{\left|z_{k}\right|} J_{\nu+1}\left(\left|z_{k}\right|\right)\right)^{2}\right. \\
& \left.+\left(\frac{-\alpha Y_{\nu}\left(\left|z_{k}\right|\right)}{\sqrt{\left|z_{k}\right|}}+\sqrt{\left|z_{k}\right|} Y_{\nu+1}\left(\left|z_{k}\right|\right)\right)^{2}\right]^{1 / 2} .
\end{aligned}
$$

\section{Esquema de Wigner}

$$
\begin{aligned}
\Psi_{\vec{k}}^{\mathrm{wig}}(\eta) & =\sqrt{\frac{\epsilon_{V}}{2}} \frac{H}{M_{P} k^{2}} \Lambda_{k}\left[F\left(k \eta, z_{k}\right) \cos \Theta_{k}\right. \\
& \left.+k G\left(k \eta, z_{k}\right) \sin \Theta_{k}\right] X_{\vec{k}} .
\end{aligned}
$$

Por otro lado, es importante escribir la expresión para el campo $\mathcal{R}(\vec{x}, \eta)$ (invariante de gauge) que representa la perturbación de la curvatura en el gauge comóvil. Su transformada de Fourier $\mathcal{R}_{\vec{k}}(\eta)$ es constante para los modos fuera del horizonte, es decir aquellos donde $k \ll \mathcal{H}=a H$. Durante inflación el valor de $\mathcal{R}_{\vec{k}}$ en el límite $k \ll \mathcal{H}$ permanece constante para todo tiempo hasta que el $k \simeq \mathcal{H}$ (lo que comúnmente se denomina entrar nuevamente al horizonte).

La perturbación de la curvatura $\Psi$ en el gauge longitudinal también es constante para todos los modos fuera del horizonte a lo largo de cualquier era cosmológica, pero no durante las transiciones, lo que lo diferencia de $\mathcal{R}$ (que permanece constante siempre). Las perturbaciones en la curvatura del gauge comóvil $\mathcal{R}$ y las correspondientes en el gauge longitudinal $\Psi$ se relacionan del siguiente modo:

$$
\mathcal{R} \equiv \Psi+(2 / 3)\left(\mathcal{H}^{-1} \Psi^{\prime}+\Psi\right) /(1+\omega),
$$

donde $\omega \equiv p / \rho$. Para los modos que cumplen la condición $k \ll \mathcal{H}$ (fuera del horizonte) durante la era inflacionaria, se puede aproximar $\omega+1 \simeq 2 \epsilon_{V} / 3$, obteniendo

$$
\lim _{k \ll \mathcal{H}} R_{\vec{k}} \simeq \lim _{k \ll \mathcal{H}} \frac{\Psi_{\vec{k}}(\eta)}{\epsilon_{V}} .
$$

Con la expresión (4.58), se puede desarrollar $\Psi_{\vec{k}}$ a orden $|k \eta|$ y encontrar la expresión de $\mathcal{R}_{\vec{k}}$ para cada uno de los esquemas de colapso. Se define

$$
X_{\vec{k}} \equiv x_{\vec{k}}^{\mathrm{R}}+i x_{\vec{k}}^{\mathrm{I}}
$$

y la amplitud

$$
R_{k} \equiv \sqrt{L^{3} \pi / \epsilon_{V}} H 2^{\nu-11 / 2} \Gamma(\nu-1) / M_{P} k^{3 / 2}
$$


donde $\Gamma(\nu-1)$ es la función gamma usual definida como $\Gamma(z)=\int_{0}^{\infty} x^{z-1} e^{x} d x$. De esta manera, se obtiene para el esquema Independiente:

$$
\mathcal{R}_{\vec{k}}^{\text {ind }} \simeq R_{k}\left[M\left(\left|z_{k}\right|\right) X_{\vec{k}, 1}+N\left(\left|z_{k}\right|\right) X_{\vec{k}, 2}\right]|k \eta|^{3 / 2-\nu}
$$

donde

$$
\begin{aligned}
M\left(\left|z_{k}\right|\right) & \equiv-\sqrt{\left|z_{k}\right|}\left[\frac{-\alpha J_{\nu}\left(\left|z_{k}\right|\right)}{\sqrt{\left|z_{k}\right|}}+\sqrt{\left|z_{k}\right|} J_{\nu+1}\left(\left|z_{k}\right|\right)\right] \\
& \times\left[J_{\nu}^{2}\left(\left|z_{k}\right|\right)+Y_{\nu}^{2}\left(\left|z_{k}\right|\right)\right]^{1 / 2} \\
N\left(\left|z_{k}\right|\right) & \equiv \sqrt{\left|z_{k}\right|} J_{\nu}\left(\left|z_{k}\right|\right) \times\left[\left(\frac{-\alpha J_{\nu}\left(\left|z_{k}\right|\right)}{\sqrt{\left|z_{k}\right|}}+\sqrt{\left|z_{k}\right|} J_{\nu+1}\left(\left|z_{k}\right|\right)\right)^{2}\right. \\
& \left.+\left(\frac{-\alpha Y_{\nu}\left(\left|z_{k}\right|\right)}{\sqrt{\left|z_{k}\right|}}+\sqrt{\left|z_{k}\right|} Y_{\nu+1}\left(\left|z_{k}\right|\right)\right)^{2}\right]^{1 / 2} .
\end{aligned}
$$

Mientras que para el esquema de Newton la expresión resulta:

$$
\mathcal{R}_{\vec{k}}^{\text {newt }} \simeq R_{k} N\left(\left|z_{k}\right|\right) X_{\vec{k}, 2}|k \eta|^{3 / 2-\nu},
$$

donde $N\left(\left|z_{k}\right|\right)$ corresponde a la expresión (4.63).

Finalmente, para el esquema de Wigner se encuentra la siguiente expresión:

$$
\mathcal{R}_{\vec{k}}^{\text {wig }} \simeq R_{k} W\left(\left|z_{k}\right|\right) X_{\vec{k}}|k \eta|^{3 / 2-\nu}
$$

donde

$$
\begin{gathered}
W\left(\left|z_{k}\right|\right) \equiv \frac{2 k^{1 / 2}}{\pi^{1 / 2} L^{3 / 2}} \times\left[-\left(\frac{-\alpha J_{\nu}\left(\left|z_{k}\right|\right)}{\sqrt{\left|z_{k}\right|}}+\sqrt{\left|z_{k}\right|} J_{\nu+1}\left(\left|z_{k}\right|\right)\right) \Lambda_{k} \cos \Theta_{k}\right. \\
\left.+\sqrt{\left|z_{k}\right|} J_{\nu}\left(\left|z_{k}\right|\right) \Lambda_{k} \sin \Theta_{k}\right] .
\end{gathered}
$$

Estas expresiones permiten vincular la perturbación inicial de la curvatura, asociada a las anisotropías en temperatura del FCR, con los parámetros que caracterizan cada esquema de colapso, el tiempo de colapso y las variables aleatorias. Estas últimas son fijadas para cada esquema una vez que tiene lugar el colapso, es decir que si se conociera de algún modo su valor podría determinarse $\mathcal{R}_{\vec{k}}$. Como las variable aleatorias son desconocidas, será necesario utilizar propiedades estadísticas para estimar observables.

\subsubsection{Cálculo del espectro primordial de fluctuaciones}

En el marco del modelo cosmológico estándar, el espectro primordial de potencias para las perturbaciones escalares tiene la forma $P(k)=A_{s} k^{n_{s}-1}$, siendo $A_{s}$ la amplitud y $n_{s}$ el índice espectral de las perturbaciones escalares. También puede encontrarse en la literatura una definición adimensional del espectro de potencias $\mathcal{P}(k)$, donde $P(k) \equiv\left(k^{3} / 2 \pi^{2}\right) \mathcal{P}(k)$ es la relación entre ambas. El formalismo del colapso permitirá desarrollar una expresión similar en la que puedan identificarse cada uno de estos términos para poder llevar adelante una comparación entre ambos modelos. Se busca encontrar una relación entre las cantidades observacionales y los parámetros que caracterizan el colapso. 
Dada la naturaleza esférica de las anisotropías en la temperatura del FCR, se descomponen para su análisis en armónicos esféricos:

$$
\frac{\delta T(\theta, \varphi)}{T_{0}}=\sum_{l m} a_{l m} Y_{l m}(\theta, \varphi)=\Theta(\hat{n})
$$

donde los coeficientes del desarrollo pueden escribirse:

$$
a_{l m}=\int \Theta(\hat{n}) Y_{l m}^{\star}(\theta, \varphi) d \Omega
$$

siendo $\hat{n}=(\sin \theta \sin \varphi, \sin \theta \cos \varphi, \cos \theta)$ una dirección en la esfera celeste tal que $\theta, \varphi$ son sus coordenadas angulares.

La relación entre las perturbaciones primordiales y las anisotropías en temperatura del FCR, asumiendo que la recombinación de produce de manera instantánea, es

$$
\Theta(\hat{n})=\left[\Psi+\frac{1}{4} \delta_{\gamma}\right]\left(\eta_{D}\right)+\hat{n} \cdot \vec{v}_{\gamma}\left(\eta_{D}\right)+2 \int_{\eta_{D}}^{\eta_{0}} \Psi^{\prime}(\eta) d \eta
$$

donde $\eta_{D}$ es el tiempo propio del desacople, $\delta_{\gamma}$ y $\vec{v}_{\gamma}$ son las perturbaciones en la densidad y radiación, respectivamente, del fluido que conforma el Universo primitivo.

También es usual trabajar con su descomposición en modos de Fourier:

$$
\Theta(\hat{n})=\sum_{\vec{k}} \frac{\Theta(\vec{k})}{L^{3}} e^{i \vec{k} \cdot R_{D} \hat{n}},
$$

siendo $R_{D}$ el radio de la superficie de último scattering.

Utilizando la relación $e^{i \vec{k} \cdot R_{D} \hat{n}}=4 \pi \sum_{l m} i^{l} j_{l}\left(k R_{D}\right) Y_{l m}(\theta, \varphi) Y_{l m}^{\star}(\hat{k})$, los coeficientes del desarrollo en armónicos esféricos pueden escribirse:

$$
a_{l m}=\frac{4 \pi i^{l}}{L^{3}} \sum_{\vec{k}} j_{l}\left(k R_{D}\right) Y_{l m}^{\star}(\hat{k}) \Theta(\vec{k}),
$$

donde $j_{l}\left(k R_{D}\right)$ son las funciones esféricas de Bessel de orden $l$.

Las anisotropías en la temperatura $\Theta(\vec{k})$ están relacionadas con las perturbaciones en la curvatura durante inflación $\mathcal{R}_{\vec{k}}$ mediante funciones de transferencia $T(k)$ :

$$
\Theta(\vec{k})=T(k) \mathcal{R}_{\vec{k}} .
$$

Estas funciones de transferencia describen la física del Universo entre el final de la etapa inflacionaria hasta la actualidad. Se pueden calcular mediante la integración numérica de las ecuaciones que describen la dinámica de cada una de las partículas presentes en el Universo junto con la ecuación de la métrica.

De este modo, los coeficientes $a_{l m}$ en términos de los modos $\mathcal{R}_{\vec{k}}$ están dados por

$$
a_{l m}=\frac{4 \pi i^{l}}{L^{3}} \sum_{\vec{k}} j_{l}\left(k R_{D}\right) Y_{l m}^{\star}(\hat{k}) T(k) \mathcal{R}_{\vec{k}},
$$

con $\mathcal{R}_{\vec{k}}$ correspondiendo al período de inflación y teniendo en cuenta el límite $k \ll \mathcal{H}$ (o su equivalente $|k \eta| \ll 1)$. 
De las expresiones de $\mathcal{R}_{\vec{k}}$ para cada esquema de colapso, puede verse que hay una relación directa entre los coeficientes $a_{l m}$ y las variables aleatorias $X_{\vec{k}}$, resultando una sumatoria sobre $\vec{k}$ de las mismas. Siendo que $X_{\vec{k}}$ son números complejos y los $a_{l m}$ son el resultado de una sumatoria sobre $\vec{k}$, se obtiene finalmente lo que en estadística se caracteriza como una caminata aleatoria de dos variables en el plano complejo. Si bien no se puede dar una estimación de la dirección final del desplazamiento, se puede estimar la intensidad del mismo, lo cual se asocia con la magnitud $\left|a_{l m}\right|^{2}$. De este modo puede estimarse su valor más probable $\left|a_{l m}\right|_{\mathrm{ML}}^{2}$ e interpretar esta cantidad como la predicción teórica de las cantidades observables. Además, como el colapso está descripto por un proceso estocástico, puede considerarse un conjunto de posibles realizaciones del mismo. Si las variables aleatorias son gaussianas, puede identificarse el valor más probable con el valor medio de todas las realizaciones posibles:

$$
\left|a_{l m}\right|_{\mathrm{ML}}^{2} \equiv \overline{\left|a_{l m}\right|^{2}} \text {. }
$$

Los valores de $\overline{\left|a_{l m}\right|^{2}}$ se pueden calcular para cada esquema de colapso. En el caso del esquema Independiente se obtiene:

$$
\begin{aligned}
\left|a_{l m}\right|_{\mathrm{ML}}^{2 \text { ind }} & =16 \pi^{2} \frac{\mathcal{C}}{L^{3}} \sum_{\vec{k}, \vec{k}^{\prime}} \frac{j_{l}\left(k R_{D}\right) j_{l}\left(k^{\prime} R_{D}\right)}{k^{3 / 2} k^{\prime 3 / 2}} \times Y_{l m}^{\star}(\hat{k}) Y_{l m}\left(\hat{k}^{\prime}\right) T(k) T\left(k^{\prime}\right) \\
& \times\left(M\left(\left|z_{k}\right|\right) M\left(\left|z_{k^{\prime}}\right|\right) \overline{X_{\vec{k}, 1} X_{\vec{k}^{\prime}, 1}^{\star}}+N\left(\left|z_{k}\right|\right) N\left(\left|z_{k^{\prime}}\right|\right) \overline{X_{\vec{k}, 2} X_{\vec{k}^{\prime}, 2}^{\star}}\right)\left(k k^{\prime}\right)^{3 / 2-\nu},
\end{aligned}
$$

donde $\mathcal{C}$ se define como:

$$
\mathcal{C} \equiv \frac{\pi}{M_{P}^{2} \epsilon_{H}}\left(2^{\nu-11 / 2} \Gamma(\nu-1) H|\eta|^{3 / 2-\nu}\right)^{2} .
$$

En el esquema de Newton la expresión está dado por:

$$
\begin{aligned}
\left|a_{l m}\right|_{\mathrm{ML}}^{2 \text { newt }} & =16 \pi^{2} \frac{\mathcal{C}}{L^{3}} \sum_{\vec{k}, \vec{k}^{\prime}} \frac{j_{l}\left(k R_{D}\right) j_{l}\left(k^{\prime} R_{D}\right)}{k^{3 / 2} k^{\prime 3 / 2}} \times Y_{l m}^{\star}(\hat{k}) Y_{l m}\left(\hat{k}^{\prime}\right) T(k) T\left(k^{\prime}\right) N\left(\left|z_{k}\right|\right) N\left(\left|z_{k^{\prime}}\right|\right) \\
& \times \bar{X}_{\vec{k}, 2} X_{\vec{k}^{\prime}, 2}^{\star}\left(k k^{\prime}\right)^{3 / 2-\nu} .
\end{aligned}
$$

Finalmente, para el esquema de Wigner se encuentra que:

$$
\begin{aligned}
\left|a_{l m}\right|_{\mathrm{ML}}^{2 \text { wig }} & =16 \pi^{2} \frac{\mathcal{C}}{L^{3}} \sum_{\vec{k}, \vec{k}^{\prime}} \frac{j_{l}\left(k R_{D}\right) j_{l}\left(k^{\prime} R_{D}\right)}{k^{3 / 2} k^{\prime 3 / 2}} \times Y_{l m}^{\star}(\hat{k}) Y_{l m}\left(\hat{k}^{\prime}\right) T(k) T\left(k^{\prime}\right) \\
& \times W\left(\left|z_{k}\right|\right) W\left(\left|z_{k^{\prime}}\right|\right) \overline{X_{\vec{k}} X_{\vec{k}^{\prime}}^{\star}}\left(k k^{\prime}\right)^{3 / 2-\nu} .
\end{aligned}
$$

Para proseguir, se necesita hacer una suposición más: las variables $x_{\vec{k}}^{R, I}$ no están correlacionadas. Entonces satisfacen:

$$
\overline{x_{\vec{k}, s}^{R} x_{\vec{k}^{\prime}, s}^{R}}=\delta_{\vec{k}, \vec{k}^{\prime}}+\delta_{\vec{k},-\vec{k}^{\prime}}, \quad \overline{x_{\vec{k}, s}^{I} x_{\vec{k}^{\prime}, s}^{I}}=\delta_{\vec{k}, \vec{k}^{\prime}}-\delta_{\vec{k},-\vec{k}^{\prime}}
$$

donde $s$ está indicando un esquema particular asociado a esa variable aleatoria.

Para poder vincular la teoría con las observaciones, se busca estimar a través de los desarrollos teóricos las cantidades observables de temperatura y polarización descriptas en la sección 1.7 .

La relación con los $a_{l m}$ en términos la ecuación (4.74) se puede expresar:

$$
C_{l}=(2 l+1)^{-1} \sum_{m}\left|a_{l m}\right|_{M L}^{2}
$$


Esto permite dar una predicción de $\operatorname{los} C_{l}$ para cada esquema de colapso, que se puede condensar en

$$
C_{l}=4 \pi \int_{0}^{\infty} \frac{d k}{k} j_{l}^{2}\left(k R_{D}\right) T(k)^{2} \frac{\mathcal{C}}{\pi^{2}} Q\left(\left|z_{k}\right|\right) k^{3-2 \nu} .
$$

Estas expresiones ya reflejan el límite al $L \rightarrow \infty$ y $\vec{k} \rightarrow$ continuo, transformando las sumas discretas sobre $\vec{k}$ en integrales. La función $Q\left(\left|z_{k}\right|\right)$ es diferente para cada esquema de colapso. Para el esquema Independiente se obtiene:

$$
Q\left(\left|z_{k}\right|\right)^{\text {ind }}=M^{2}\left(\left|z_{k}\right|\right)+N^{2}\left(\left|z_{k}\right|\right)
$$

mientras que para el esquema de Newton:

$$
Q\left(\left|z_{k}\right|\right)^{\text {newt }}=N^{2}\left(\left|z_{k}\right|\right)
$$

y para el de Wigner:

$$
Q\left(\left|z_{k}\right|\right)^{\text {wig }}=W^{2}\left(\left|z_{k}\right|\right) .
$$

En cada caso, las definiciones de $M, N$ y $W$ corresponden a las ecuaciones (4.62), (4.63) y (4.66) respectivamente.

En el modelo cosmológico estándar, el espectro de potencias $P(k)$ para las perturbaciones $\mathcal{R}_{\vec{k}}$ y los coeficientes $C_{l}$ están relacionados del siguiente modo:

$$
C_{l}=4 \pi \int_{0}^{\infty} \frac{d k}{k} j_{l}^{2}\left(k R_{D}\right) T(k)^{2} P(k) .
$$

Al comparar las ecuaciones (4.81) y (4.85) puede identificarse un espectro de potencias equivalente para cada esquema de colapso, que puede escribirse genéricamente:

$$
P(k)=\frac{\mathcal{C}}{\pi^{2}} Q\left(\left|z_{k}\right|\right) k^{3-2 \nu},
$$

donde $Q\left(\left|z_{k}\right|\right)$ debe reemplazarse por el correspondiente para cada esquema. Estos resultados permiten finalmente obtener una predicción teórica de los observables susceptible de compararse con los datos.

En esta instancia, vale la pena detenerse a aclarar algunos detalles comparando el procedimiento realizado en el marco del colapso respecto al modelo cosmológico estándar. Para obtener el espectro primordial de potencias en el modelo de inflación estándar se calcula la función de correlación cuántica de dos puntos. Siendo $\hat{\Psi}$ un operador cuántico asociado a las perturbaciones de la métrica, el espectro de perturbaciones escalares se obtiene del siguiente modo:

$$
\left\langle 0\left|\hat{\Psi}_{\vec{k}} \hat{\Psi}_{\vec{k}^{\prime}}\right| 0\right\rangle=\frac{2 \pi^{2}}{k^{3}} P(k) \delta\left(\vec{k}-\vec{k}^{\prime}\right) .
$$

Sin embargo, no hay que perder de vista que la definición del espectro de potencias está dada en términos de $\Psi_{\vec{k}}$ un campo clásico estocástico, no un operador cuántico. Esto implica que el enfoque estándar requiere hacer la identificación

$$
\left\langle 0\left|\hat{\Psi}_{\vec{k}} \hat{\Psi}_{\vec{k}^{\prime}}\right| 0\right\rangle=\overline{\Psi_{\vec{k}} \Psi_{\vec{k}^{\prime}}},
$$

donde $\overline{\Psi_{\vec{k}} \Psi_{\overrightarrow{k^{\prime}}}}$ es un promedio sobre un ensamble de campos clásicos estocásticos. La justificación para tal procedimiento suele basarse en argumentos derivados de la decoherencia o que los estados cuánticos de vacío de inflación evolucionan naturalmente hacia un estado comprimido (squeezed states) (Grishchuk \& Sidorov, 1990; Kiefer \& Polarski, 2009). Una 
amplia discusión acerca de su validez en el contexto cosmológico y la aplicación al período inflacionario está descripta en los trabajos de Landau et al. (2013) y Sudarsky (2011). De todos modos, ningún mecanismo de los antes mencionados puede explicar la ruptura de las simetrías del estado de vacío inicial y, por ende, el surgimiento de las perturbaciones en la curvatura $\Psi_{\vec{k}}$.

Es importante destacar que el espectro de potencias obtenido para los modelos de colapso se calcula en base a

$$
\overline{\left\langle\Theta\left|\pi_{\vec{k}}\right| \Theta\right\rangle\left\langle\Theta\left|\hat{\pi}_{\vec{k}^{\prime}}\right| \Theta\right\rangle^{*}},
$$

sin hacer una identificación de los operadores cuánticos con funciones clásicas. Para construir el espectro de potencias no se recurrió a la función de correlación de dos puntos si no a un producto de correlaciones de un solo punto, identificando luego el espectro de potencias equivalente mediante la comparación directa de ambas expresiones.

\subsubsection{Comparación entre los espectros de potencias}

Las cantidades observables del FCR son los coeficientes de las anisotropías, que dependen tanto del espectro de potencias $P(k)$ determinado por inflación como de la función de transferencia $\mathcal{T}$ que da cuenta de la evolución desde el final de inflación hasta nuestros días:

$$
C_{l}=\frac{2}{\pi} \int P(k) \mathcal{T}^{2}(k) \frac{d k}{k} .
$$

La expresión fenomenológica más general que se suele considerar para $P(k)$ está dada por un desarrollo en potencias de $k$ :

$$
P(k)=A_{s}\left(\frac{k}{k_{\star}}\right)^{n s-1+\frac{1}{2} n_{\text {run }} \ln \left(\frac{k}{k_{\star}}\right)},
$$

donde $k_{\star}$ es una escala de referencia (usualmente $k_{\star}=0.05 \mathrm{Mpc}^{-1}$ ); y siendo

$$
\begin{aligned}
n_{s}-1 & =\frac{d \ln (P(k))}{d \ln (k)}, \\
n_{\text {run }} & =\frac{d n_{s}}{d \ln (k)}=\frac{d^{2} \ln (P(k))}{d(\ln (k))^{2}},
\end{aligned}
$$

siendo $n_{\text {run }}$ parte de la ampliación de un desarrollo a segundo orden en los parámetros de slow-roll. En esta tesis se considera el caso $n_{\text {run }}=0$, debido a que las dependencias de $k$ que no sean de la forma de potencias estarán dadas por la física del colapso y se incluirán en una función adicional. Los parámetros $A_{s}$ y $n_{s}$ se definen del siguiente modo:

$$
\begin{aligned}
A_{s} & =\frac{2^{2 \tilde{\nu}-4}|\Gamma(\tilde{\nu})|^{2} H^{2}|\eta|^{3-2 \tilde{\nu}}}{\pi^{3} M_{P}^{2} \epsilon_{H}}, \\
n_{s}-1 & =-6 \epsilon_{H}+2 \delta_{V} .
\end{aligned}
$$

Por otro lado, la ecuación (4.25) indica que para los modelos de colapso abordados en esta sección $\nu=3 / 2+\epsilon_{H}-\delta_{V}$. Se puede mostrar que $A_{s}$ es una función aproximadamente constante (Kinney (2005)). Es usual expresar $P(k)$ en términos de cantidades evaluadas al momento en que el modo cruza el horizonte $k=a H$. Así el espectro de potencias, a primer orden en los parámetros de slow-roll, se escribe en términos del número de onda $k$ y es independiente del tiempo:

$$
P(k)=\frac{2^{2 \tilde{\nu}-4}|\Gamma(\tilde{\nu})|^{2}}{\pi^{3} M_{P}^{2}} \frac{H_{\star}^{2}(k)}{\epsilon_{H}^{\star}(k)},
$$


donde

$$
\left.\frac{H_{\star}^{2}(k)}{\epsilon_{H}^{\star}(k)} \equiv\left(\frac{H^{2}|\eta|^{3-2 \tilde{\nu}}}{\epsilon_{H}}\right)\right|_{k=a H} .
$$

El índice espectral se calcula como $n_{s}-1=d \ln P(k) / d \ln k$ con las ecuaciones anteriores.

Por otro lado el espectro de potencias equivalente para los esquemas de colapso es $P(k)=$ $\mathcal{C} / \pi^{2} Q\left(\left|z_{k}\right|\right) k^{3-2 \nu}$ (ecuación (4.86)), donde $\mathcal{C}$ (ecuación (4.76)) tiene la misma estructura que $A_{s}$ (ecuación (4.93)). Esto quiere decir que también es aproximadamente independiente del tiempo o, dicho de otro modo, su derivada respecto al tiempo conforme es una función de segundo orden en los parámetros de slow-roll. Continuar con el procedimiento estándar implicaría evaluar $\mathcal{C}$ al momento en que los modos cruzan el horizonte, sin embargo el enfoque del colapso indica que las perturbaciones en la curvatura, y por ende el espectro de potencias, surgen al momento del colapso, siendo nulas previamente.

Una vez que el mecanismo de colapso ha llegado a su fin y se recupera la validez de la aproximación semiclásica para algún tiempo $\eta_{\vec{k}}^{c}$ durante inflación, $\Psi_{\vec{k}} \neq 0, \forall \vec{k}$ generando las primeras perturbaciones en la curvatura. De este modo, puede tomarse un modo de Fourier en particular y analizar si su longitud de onda propia asociada $\lambda_{P}=a / k$ (donde $k$ es el número de onda comóvil) al momento de colapso es mayor o menor que el radio de Hubble (recordar la definición de la página 42).

Analizar las dependencias en $Q\left(\left|z_{k}\right|\right)$ darán algunas pautas más para caracterizar al mecanismo de colapso. Este depende de la variable $z_{k}$ que es función de $k\left(z_{k} \equiv k \eta_{\vec{k}}^{c}\right)$. Si el tiempo de colapso es de la forma $\eta_{\vec{k}}^{c} \propto 1 / k$ entonces $z_{k}$ no depende de $k$ y $Q\left(\left|z_{k}\right|\right)$ será una constante, por lo que el espectro primordial de potencias será de la forma $P(k) \propto k^{3-2 \nu}$. Las predicciones observacionales derivadas de tal espectro son indistinguibles de las del modelo cosmológico estándar.

Si el espectro de potencias se expresa de la forma $P(k)=\mathcal{A} k^{n_{s}-1}$, pueden compararse ambos modelos resultando la siguiente identificación ${ }^{5}$ :

$$
\begin{aligned}
\mathcal{A} & =\frac{2^{2 \nu-11}|\Gamma(\nu-1)|^{2} H^{2}|\eta|^{3-2 \nu}}{\pi M_{P}^{2} \epsilon_{H}} Q(|z|), \\
n_{s}-1 & =-2 \epsilon_{H}+2 \delta_{V} .
\end{aligned}
$$

Una propuesta posible para la parametrización del tiempo de colapso es permitir una pequeña dependencia en $k$ a través de un pequeño apartamiento:

$$
\eta_{\vec{k}}^{c}=\frac{A}{k}+B
$$

Existen otras propuestas donde, por ejemplo, $\eta_{\vec{k}}^{c}=\frac{A}{k}+\frac{B}{k^{2}}$. La diferencia entre estos dos tipos de parametrizaciones es que en la primera los distintos valores de $B$ producen apartamientos en los multipolos más grandes, mientras que la segunda propuesta modifica las predicciones para los multipolos más pequeños.

\subsubsection{Esquemas dentro y fuera del radio de Hubble}

A continuación se calculan las expresiones del espectro primordial de potencias en el caso para el cual el colapso tiene lugar cuando la longitud de onda propia comóvil asociada al modo es mayor o menor al radio de Hubble comóvil (fuera/dentro del "horizonte" respectivamente).

\footnotetext{
${ }^{5}$ Recordar que en el marco del modelo cosmológico estándar $n_{s}-1=-6 \epsilon_{H}+2 \delta_{V}$
} 
Si la longitud de onda propia del modo al momento del colapso es más grande que el radio de Hubble, entonces:

$$
k \ll a\left(\eta_{\vec{k}}^{c}\right) H=\mathcal{H}\left(\eta_{\vec{k}}^{c}\right) \simeq-1 / \eta_{\vec{k}}^{c},
$$

o equivalentemente $-k \eta_{\vec{k}}^{c} \ll 1$, y el espectro de potencias puede aproximarse por

$$
P(k) \simeq \frac{\mathcal{C}}{\pi^{2}} \Xi\left(\left|z_{k}\right|\right) k^{3-2 \nu},
$$

donde $\Xi\left(\left|z_{k}\right|\right)$ es el resultado de expandir la función $Q\left(\left|z_{k}\right|\right)$ al orden más bajo en $\left|z_{k}\right|$ (en este caso $\left.\left|z_{k}\right| \rightarrow 0\right)$. Para cada uno de los distintos esquemas se obtiene:

$$
\begin{gathered}
\Xi\left(\left|z_{k}\right|\right)^{\text {ind }} \equiv \frac{4}{\pi^{2}}\left[1+\frac{\left|z_{k}\right|^{2}}{2}\left(\frac{1}{\nu-1}-\frac{1}{\nu}\right)\right] \\
\Xi\left(\left|z_{k}\right|\right)^{\text {newt }} \equiv \frac{\left|z_{k}\right|^{4}}{4 \pi^{2} \nu^{2}(\nu-1)^{2}}, \\
\Xi\left(\left|z_{k}\right|\right)^{\text {wig }} \equiv \frac{16}{\pi} \times\left[\frac{5}{4}+\frac{1}{4\left|z_{k}\right|^{2}}\left(1-\sqrt{1+10\left|z_{k}\right|^{2}+9\left|z_{k}\right|^{4}}\right)\right]^{-2} \\
\times\left[\frac{\left|z_{k}\right|^{\nu-1 / 2}}{\Gamma(\nu) 2^{\nu-1}} \cos \Theta_{k}+\frac{\left|z_{k}\right|^{\nu+1 / 2}}{\Gamma(\nu-1) 2^{\nu}} \sin \Theta_{k}\right]^{2}
\end{gathered}
$$

donde $\tan 2 \Theta_{k} \simeq 4\left|z_{k}\right| /\left(1-3\left|z_{k}\right|^{2}\right)$.

$\mathrm{Si}$ en cambio, la longitud de onda propia asociada al modo es menor que el radio de Hubble al momento del colapso, entonces

$$
k \gg a\left(\eta_{\vec{k}}^{c}\right) H
$$

lo que equivale a $-k \eta_{\vec{k}}^{c} \gg 1$. Esto implica poder aproximar el espectro de potencias de los modelos de colapso cuando $-k \eta_{\vec{k}}^{c}=\left|z_{k}\right| \rightarrow \infty$, resultando:

$$
P(k) \simeq \frac{\mathcal{C}}{\pi^{2}} \Upsilon\left(\left|z_{k}\right|\right) k^{3-2 \nu},
$$

donde $\Upsilon\left(\left|z_{k}\right|\right)$ es el resultado de considerar el comportamiento asintótico de la función $Q\left(\left|z_{k}\right|\right)$, y para cada esquema toma la forma:

$$
\begin{aligned}
\Upsilon\left(\left|z_{k}\right|\right)^{\text {ind }} & \equiv \frac{4}{\pi^{2}}\left\{\left[1+\frac{1}{4\left|z_{k}\right|^{2}}\left(\frac{\Gamma(\nu+3 / 2)}{\Gamma(\nu-1 / 2)}\right)^{2}\right]\right. \\
& \times\left[\sin \beta\left(\nu,\left|z_{k}\right|\right)+\frac{\cos \beta\left(\nu,\left|z_{k}\right|\right)}{\left|z_{k}\right|}\right. \\
& \times\left(-2 \nu+\frac{\Gamma(\nu+5 / 2)}{2 \Gamma(\nu+1 / 2)}\right]^{2}+\left[1+\frac{1}{\left|z_{k}\right|^{2}}\right. \\
& \left.\times\left(-2 \nu+\frac{\Gamma(\nu+5 / 2)}{2 \Gamma(\nu+1 / 2)}\right)^{2}\right] \\
& \left.\times\left[\cos \beta\left(\nu,\left|z_{k}\right|\right)-\frac{\sin \beta\left(\nu,\left|z_{k}\right|\right)}{2\left|z_{k}\right|} \frac{\Gamma(\nu+3 / 2)}{\Gamma(\nu-1 / 2)}\right]^{2}\right\},
\end{aligned}
$$




$$
\begin{aligned}
\Upsilon\left(\left|z_{k}\right|\right)^{\text {newt }} & \equiv \frac{4}{\pi^{2}} \\
& \times\left[1+\frac{1}{\left|z_{k}\right|^{2}}\left(-2 \nu+\frac{\Gamma(\nu+5 / 2)}{2 \Gamma(\nu+1 / 2)}\right)^{2}\right] \\
& \times\left[\cos \beta\left(\nu,\left|z_{k}\right|\right)-\frac{\sin \beta\left(\nu,\left|z_{k}\right|\right)}{2\left|z_{k}\right|} \frac{\Gamma(\nu+3 / 2)}{\Gamma(\nu-1 / 2)}\right]^{2}, \\
\Upsilon\left(\left|z_{k}\right|\right)^{\text {wig }} \equiv & \frac{16}{\pi^{2}}\left\{\left[\frac{2 \nu}{\left|z_{k}\right|^{3 / 2}}\right.\right. \\
\times & \left(\cos \beta\left(\nu,\left|z_{k}\right|\right)-\frac{\sin \beta\left(\nu,\left|z_{k}\right|\right)}{2\left|z_{k}\right|} \frac{\Gamma(\nu+3 / 2)}{\Gamma(\nu-1 / 2)}\right) \\
- & \left.\left(\sin \beta\left(\nu,\left|z_{k}\right|\right)+\frac{\cos \beta\left(\nu,\left|z_{k}\right|\right)}{2\left|z_{k}\right|} \frac{\Gamma(\nu+5 / 2)}{\Gamma(\nu+1 / 2)}\right)\right] \cos \Theta_{k} \\
+ & {\left.\left[\cos \beta\left(\nu,\left|z_{k}\right|\right)-\frac{\sin \beta\left(\nu,\left|z_{k}\right|\right)}{2\left|z_{k}\right|} \frac{\Gamma(\nu+3 / 2)}{\Gamma(\nu-1 / 2)}\right] \sin \Theta_{k}\right\}^{2}, }
\end{aligned}
$$

donde $\beta\left(\nu,\left|z_{k}\right|\right) \equiv\left|z_{k}\right|-(\pi / 2)(\nu+1 / 2)$ y $\tan 2 \Theta_{k} \simeq-4 / 3\left|z_{k}\right|$.

Las expresiones (4.101) y (4.104) son las que se incorporarán a los códigos numéricos para obtener las predicciones teóricas de las funciones de correlación de la temperatura y polarización.

\subsubsection{Comparación del espectro primordial de los modelos de colapso con el del modelo cosmológico estándar}

Una vez descriptos los modelos de colapso en la aproximación semiclásica, el primer paso a seguir para estudiarlos es analizar el espectro primordial de potencias en cada caso.

Las figuras 4.1, 4.2 y 4.3 muestran los resultados para los tres esquemas propuestos para el caso en que el colapso se produce cuando el modo está fuera del horizonte. En cada una se muestran, a modo de referencia y comparación, los resultados de un modelo con espectro de potencias correspondiente al modelo cosmológico estándar $P(k)=A_{s} k^{n_{s}-1}$ donde $n_{s}=0.96$, que es el valor medio obtenido por la colaboración Planck Collaboration et al. (2016b) y al que se hará referencia como modelo fiducial.

Las líneas que representan el modelo estándar y los modelos de colapso han sido deliberadamente separadas para visualizar mejor la referencia. Su alejamiento o acercamiento depende simplemente de la normalización. El espectro primordial de potencias indica una relación de las perturbaciones según la escala, por lo que el resultado importante a obtener de aquí es su dependencia funcional, en este caso, la pendiente. El tiempo conforme de colapso se parametriza como $\eta_{\vec{k}}^{c}=A / k+B$, y asignar $B=0$ recupera la forma funcional del modelo cosmológico estándar. Esto hace que la normalización global esté dada por una combinación de $A$ y $A_{s}$, lo que arroja dos conclusiones importantes: ambos parámetros están fuertemente correlacionados, y $A$ funciona como una normalización. La consecuencia más importante es que el análisis de viabilidad de los modelos de colapso no dependerá de $A$ ya que este valor será absorbido por $A_{s}$, cuya estimación se dará a través del análisis estadístico con datos observacionales. Así, se podría elegir un valor representativo para $A$ (por ejemplo aquel a partir del cual resulte una normalización global lo más similar posible al valor de $A_{s}$ correspondiente al modelo estándar) y someter únicamente a $B$ al análisis correspondiente. 
Los valores relevantes de $k$ que afectan a las predicciones de anisotropías en temperatura y polarización del FCR se encuentran en el intervalo $10^{-6} \mathrm{Mpc}^{-1}<k<10^{-1} \mathrm{Mpc}^{-1}$, por lo que cada figura cuenta con una línea indicando este límite. Hacia valores más grandes que $10^{-1} \mathrm{Mpc}$ los apartamientos respecto al espectro primordial de potencias que marca el modelo estándar no son de interés observacional.

Para el caso del esquema Independiente, la figura 4.1 para $B=-0.1 \mathrm{Mpc}$ el espectro de potencias puede solaparse perfectamente con el del modelo fiducial con una normalización adecuada. Los valores $B=-0.5 \mathrm{Mpc}$ y $B=-1 \mathrm{Mpc}$ se apartan de la pendiente que marca el modelo fiducial para $k>10^{-1} \mathrm{Mpc}^{-1}$ independientemente del valor de $A_{s}$ elegido. Sin embargo, como se destacó anteriormente, el apartamiento sucede en una escala que no es relevante para las observaciones, por lo que no se espera encontrar apartamientos en el espectro angular de anisotropías.
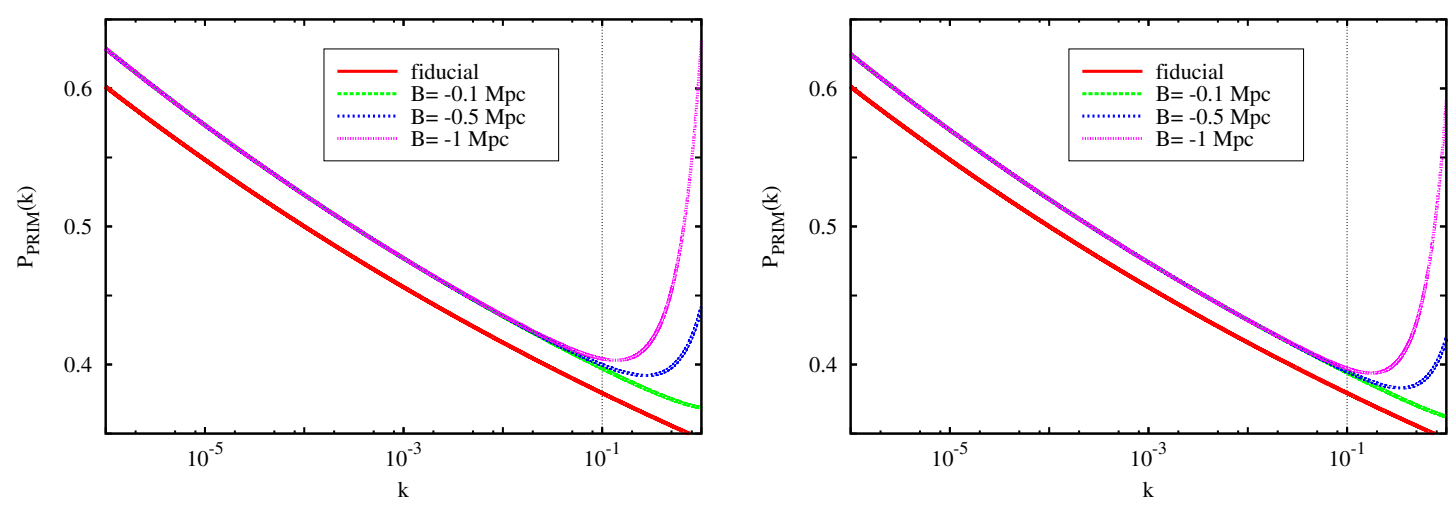

Figura 4.1. Espectro primordial de potencias para el esquema de Independiente en el caso que $k \ll \mathcal{H}\left(\eta_{\vec{k}}^{c}\right)$. Recordando que el tiempo de colapso se parametriza $\eta_{\vec{k}}^{c}=A / k+B$, el gráfico de la izquierda muestra el comportamiento para $A=-10^{-1}$ y a la derecha para $A=-10^{-2}$, en ambos casos la normalización se fija en $A_{s}=0.39$. El valor para $B$ se indica en la leyenda. León et al. (2015b).

Para el caso del esquema de Newton (figura 4.2), si bien las pendientes coinciden para valores pequeños de $k$, comienzan a presentarse desviaciones más pronunciadas $\mathrm{y}$, conforme se incrementa el valor de $B$, el desvío ocurre a escalas que ciertamente afectarán las predicciones de los espectros de anisotropías. Los valores de $A_{s}$ necesarios para lograr solapamiento entre los modelos de colapso y el modelo fiducial figuran en los epígrafes.

Para el esquema de Wigner (figura 4.3), el análisis es similar al del esquema anterior, aunque es interesante notar que la desviación se da en este caso para el lado opuesto.

En resumen, para el caso en que $k \ll \mathcal{H}\left(\eta_{\vec{k}}^{c}\right)$ este análisis preliminar muestra que existen tanto valores de $A$ como $B$ capaces de producir un espectro primordial de potencias casi idéntico a del modelo fiducial.

Las figuras 4.4, 4.5 y 4.6 (León et al., 2015b) muestran el caso de los tres esquemas propuestos en el caso en que el colapso se produce cuando el modo está dentro del horizonte $\left(k \gg \mathcal{H}\left(\eta_{\vec{k}}^{c}\right)\right)$ para distintos valores de los parámetros $A$ y $B$. Al igual que los casos anteriores, los valores de $A$ y $A_{s}$ se detallan en el correspondiente epígrafe. El espectro de potencias del esquema Independiente (figura 4.4) denota para el caso $A=-10^{2}$ un apartamiento del modelo fiducial para valores crecientes de $B$, aunque son mucho más pequeños que para otros esquemas. Para $A=-10^{6}$ no se observan apartamientos para los valores de $B$ considerados. Esto ocurre debido a que para grandes valores de $A$, el valor de $z_{k}$ también se torna grande 

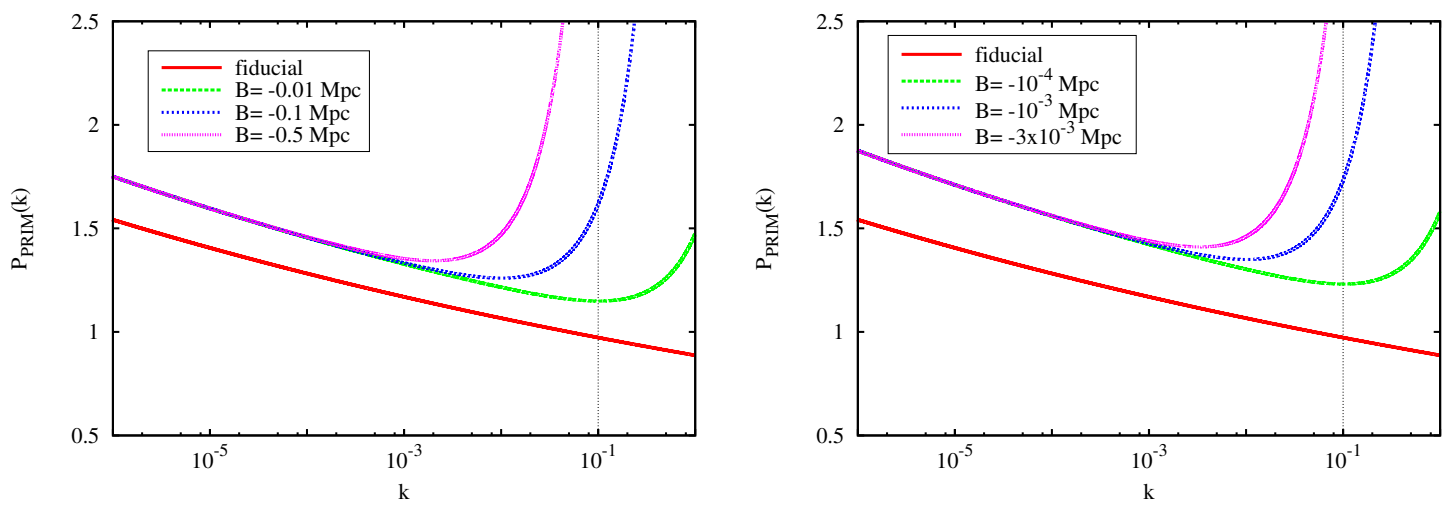

Figura 4.2. Espectro primordial de potencias para el esquema de Newton en el caso que $k \ll \mathcal{H}\left(\eta_{\vec{k}}^{c}\right)$. Recordando que el tiempo de colapso se parametriza $\eta_{\vec{k}}^{c}=A / k+B$, el gráfico de la izquierda muestra el comportamiento para $A=-10^{-1}$ y a la derecha para $A=-10^{-3}$, con una normalización de $A_{s}=2.8 \times 10^{5}$ y $A_{s}=3 \times 10^{13}$ respectivamente. El valor para $B$ se indica en la leyenda. León et al. (2015b).
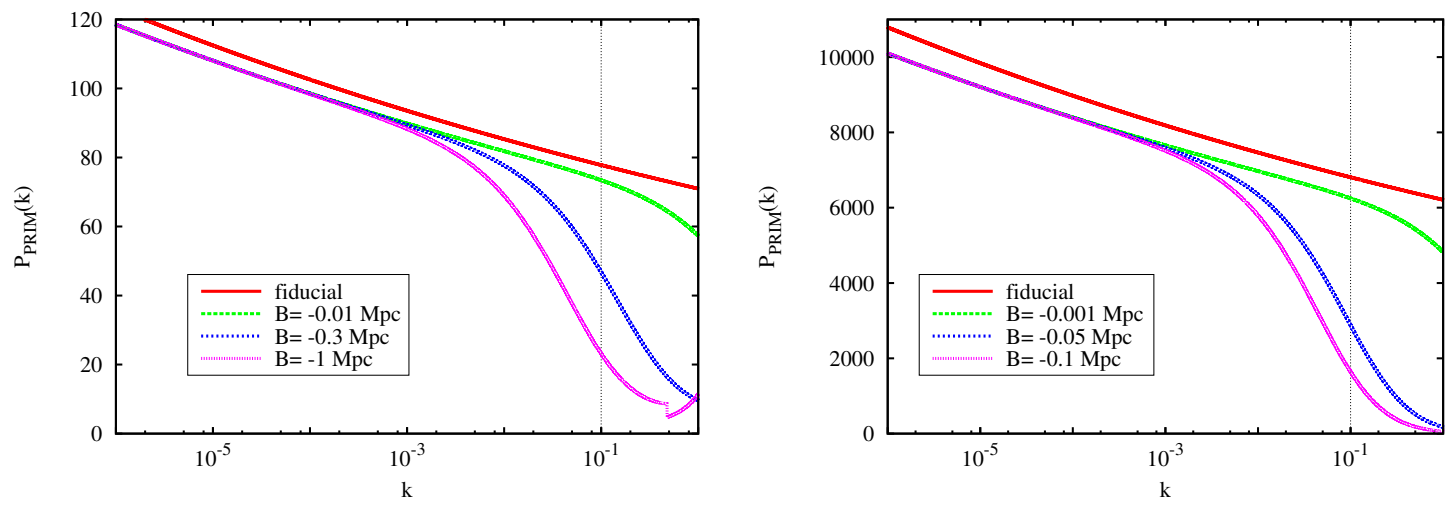

Figura 4.3. Espectro primordial de potencias para el esquema de Wigner en el caso que $k \ll \mathcal{H}\left(\eta_{\vec{k}}^{c}\right)$. Recordando que el tiempo de colapso se parametriza $\eta_{\vec{k}}^{c}=A / k+B$, el gráfico de la izquierda muestra el comportamiento para $A=-10^{-1}$ y a la derecha para $A=-10^{-2}$, con una normalización de $A_{s}=70$ y $A_{s}=7000$ respectivamente. El valor para $B$ se indica en la leyenda. León et al. (2015b)

y por lo tanto sumar el valor de un pequeño apartamiento $B$ no introduce modificaciones que afecten a la forma final del espectro de potencias (ecuación (4.105a)). Para el caso del esquema de Newton (figura 4.5) ambos valores $A=-10^{2}$ y $A=-10^{5}$ muestran desviaciones para valores crecientes de $B$ a partir de $k>0.01 \mathrm{Mpc}^{-1}$. El esquema de Wigner (figura 4.6) también muestra cada vez mayor desviación del modelo fiducial para valores increyentes de $B$ en ambos casos $\left(A=-10^{2}\right.$ y $\left.A=-10^{6}\right)$ para valores $k>0.005 \mathrm{Mpc}^{-1}$. En conclusión, valores más grandes de $B$ determinan mayores apartamientos en el espectro primordial de potencias respecto del modelo fiducial. Para los casos de Newton y Wigner es mucho mayor que para el esquema Independiente.

\subsubsection{Espectro angular de anisotropías del Fondo Cósmico de Radiación}

En esta sección se muestra el efecto de introducir el colapso de la función de onda del campo inflatón durante el período de inflación en las cantidades observables del FCR, como 

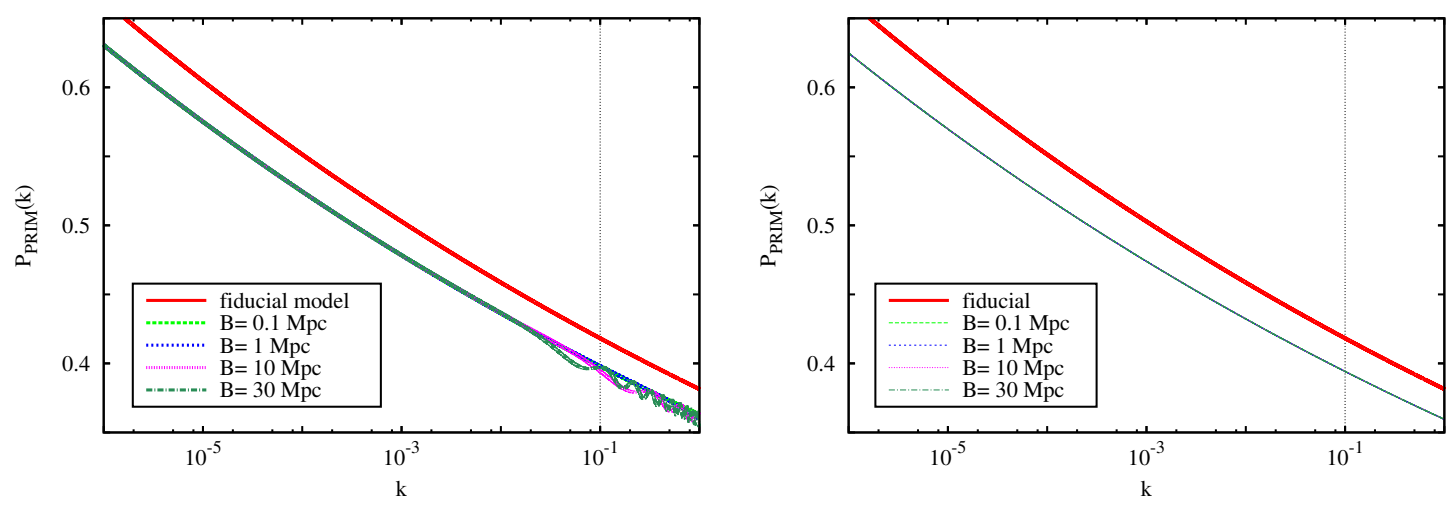

Figura 4.4. Espectro primordial de potencias para el esquema Independiente en el caso que $k \gg \mathcal{H}\left(\eta_{\vec{k}}^{c}\right)$. Recordando que el tiempo de colapso se parametriza $\eta_{\vec{k}}^{c}=A / k+B$, el gráfico de la izquierda muestra el comportamiento para $A=-10^{2}$ y a la derecha para $A=-10^{6}$, con una normalización de $A_{s}=0.43$ en ambos casos. El valor asignado a $B$ se indica en la leyenda. León et al. (2015b).
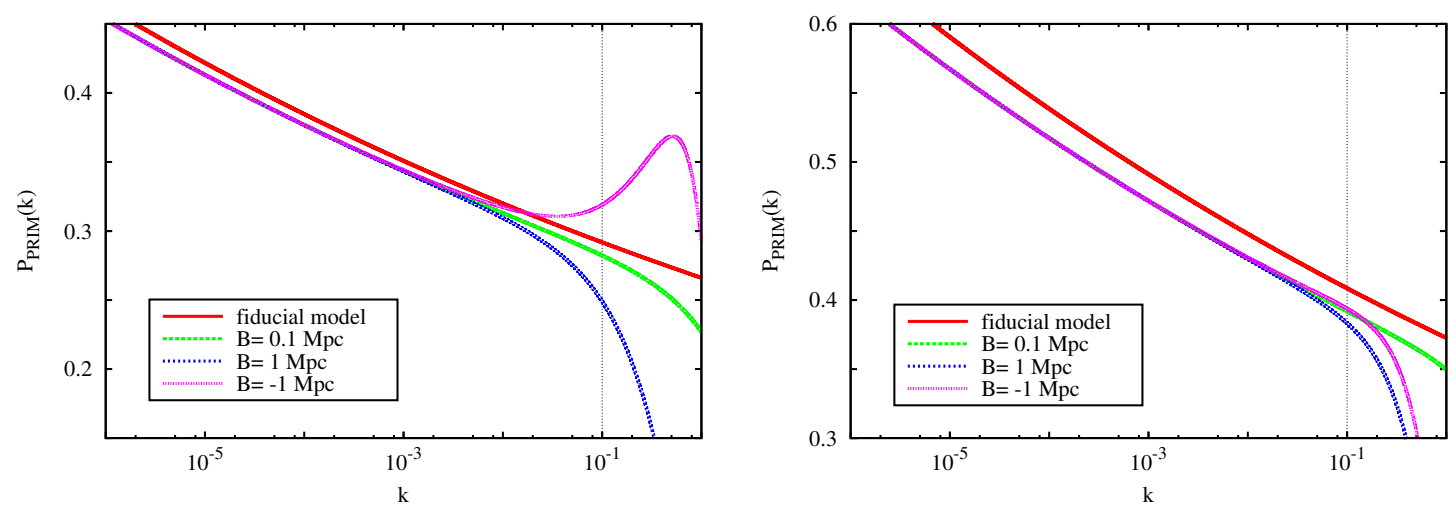

Figura 4.5. Espectro primordial de potencias para el esquema de Newton en el caso que $k \gg \mathcal{H}\left(\eta_{\vec{k}}^{c}\right)$. Recordando que el tiempo de colapso se parametriza $\eta_{\vec{k}}^{c}=A / k+B$, el gráfico de la izquierda muestra el comportamiento para $A=-10^{2}$ y a la derecha para $A=-10^{5}$, con una normalización de $A_{s}=0.3$ y $A_{s}=0.42$ respectivamente. El valor asignado a $B$ se indica en la leyenda. León et al. (2015b)

lo son los espectros de anisotropías. Las figuras 4.7, 4.8, 4.9, 4.10, 4.11 y 4.12 muestran la autocorrelación de las anisotropías en temperatura para los mismos valores de $A$ y $B$ analizados para el espectro primordial de potencias en la sección 4.4.7. El efecto es distinto para cada uno de los esquemas propuestos y depende también del valor del tiempo de colapso. La separación que marca la línea punteada vertical no representa una ventana observacional como en los casos anteriores, si no que simplemente denota un cambio en la escala del eje de las absisas: escala logarítmica para $2<l<29$ y lineal para $l>30$.

Para obtener las predicciones teóricas de los espectros de anisotropías del FCR en el caso de los distintos esquemas de colapso, es necesario modificar en el código abierto camb las expresiones de los espectros primordiales de potencias según cada esquema e incluir los parámetros libres de colapso como nuevas variables del programa.

De modo análogo a la sección anterior, se establece un modelo fiducial de referencia para realizar la comparación, definido en función del mejor ajuste de los parámetros cosmológicos obtenido por la colaboración Planck (Planck Collaboration et al., 2014b) para los datos 

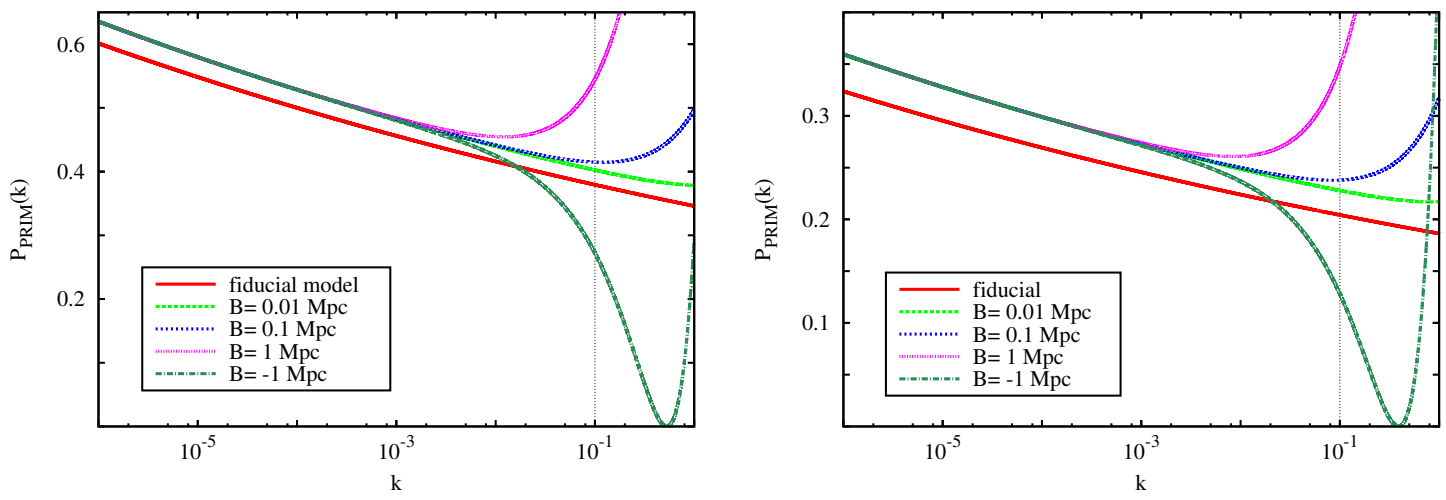

Figura 4.6. Espectro primordial de potencias para el esquema de Wigner en el caso que $k \gg \mathcal{H}\left(\eta_{\vec{k}}^{c}\right)$. El gráfico de la izquierda muestra el comportamiento para $A=-10^{2}$ y el de la derecha para $A=-10^{6}$, con una normalización de $A_{s}=0.39$ y $A_{s}=0.21$ respectivamente. El valor asignado a $B$ se indica en la leyenda. León et al. (2015b).

PlanckTT+lowP:

$$
\begin{aligned}
\Omega_{B} h^{2} & =0.02212 \text { densidad de materia bariónica en términos de la densidad crítica } \\
\Omega_{C D M} h^{2} & =0.1187 \text { densidad de materia oscura en términos de la densidad crítica } \\
H_{O} & =67.75 \text { constante de Hubble en unidades de } \mathrm{Mpc}^{-1} \mathrm{~km} / \mathrm{s} \\
\tau & =0.092 \text { profundidad óptica de reionización } \\
n_{s} & =0.96 \text { índice espectral de las perturbaciones escalares. }
\end{aligned}
$$

Cada epígrafe indica los valores de $A$ adoptados. El valor de $A_{s}$ es tal que el máximo del primer pico de las anisotropías coincida con el del modelo fiducial.

En las figuras 4.7, 4.8 y 4.9 se muestran gráficos para los esquemas Independiente, Newton y Wigner en el caso que la longitud de onda propia del modo es mayor al radio de Hubble al momento del colapso. En el caso del esquema Independiente existe muy poca desviación respecto al modelo fiducial, de hecho se espera que prácticamente cualquier valor de $B$ que cumpla con las restricciones correspondientes pueda explicar los datos observacionales.

Sin embargo para los casos de Newton y Wigner (figuras 4.8 y 4.9 respectivamente), se distinguen apartamientos notorios respecto al modelo fiducial conforme aumenta el valor de $B$. Es importante destacar que ambos esquemas necesitan valores muy altos de $A_{s}$ para lograr coincidencia en el primer pico del espectro de anisotropías. De hecho la diferencia se da hasta en varios órdenes de magnitud, por lo cual es necesario hacer un estudio previo y conocer con antelación el valor de la normalización para corregir el espectro primordial por un factor particular para cada caso de modo de salvaguardar errores numéricos en la programación.

Las figuras 4.10, 4.11 y 4.12 muestran las predicciones para el espectro de anisotropías de la temperatura en el caso de que la longitud de onda propia del modo al momento del colapso sea menor que el radio de Hubble $\left(k \gg \mathcal{H}\left(\eta_{\vec{k}}^{c}\right)\right)$. De acuerdo con la figura 4.10, el esquema Independiente no muestra cambios para los distintos valores de $B$ y los apartamientos respecto al modelo fiducial son mínimos. Se espera que cualquier valor de $B$ represente bien las observaciones actuales para los valores de $A$ analizados, razón por la cual no se incluye este esquema en los análisis estadísticos.

En el caso de Newton y Wigner (figuras 4.11 y 4.12 respectivamente) se ve cómo el valor de $B$ afecta a las predicciones de las anisotropías, los picos secundarios se separan de la línea 

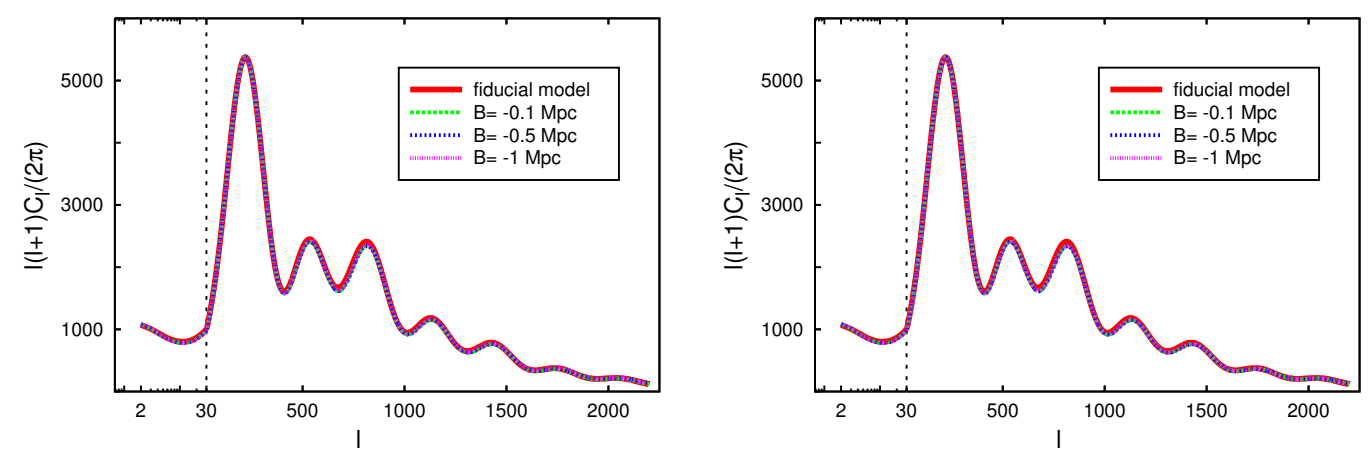

Figura 4.7. Espectro de anisotropías de la temperatura del FCR en el caso donde $k \ll$ $\mathcal{H}\left(\eta_{\vec{k}}^{c}\right)$ para el esquema Independiente. El modelo fiducial se muestra junto con distintas predicciones para este esquema de colapso según diferentes valores para $\eta_{\vec{k}}^{c}=A / k+B$. El índice espectral escalar se fijó en $n_{s}=0.96$ mientras que $A_{s}$ toma valores tal que el máximo del primer pico coincida con el del modelo fiducial. Se muestra a la izquierda los resultados para $A=-10^{-1}$ y a la derecha para $A=-10^{-2}$. León et al. (2015b).
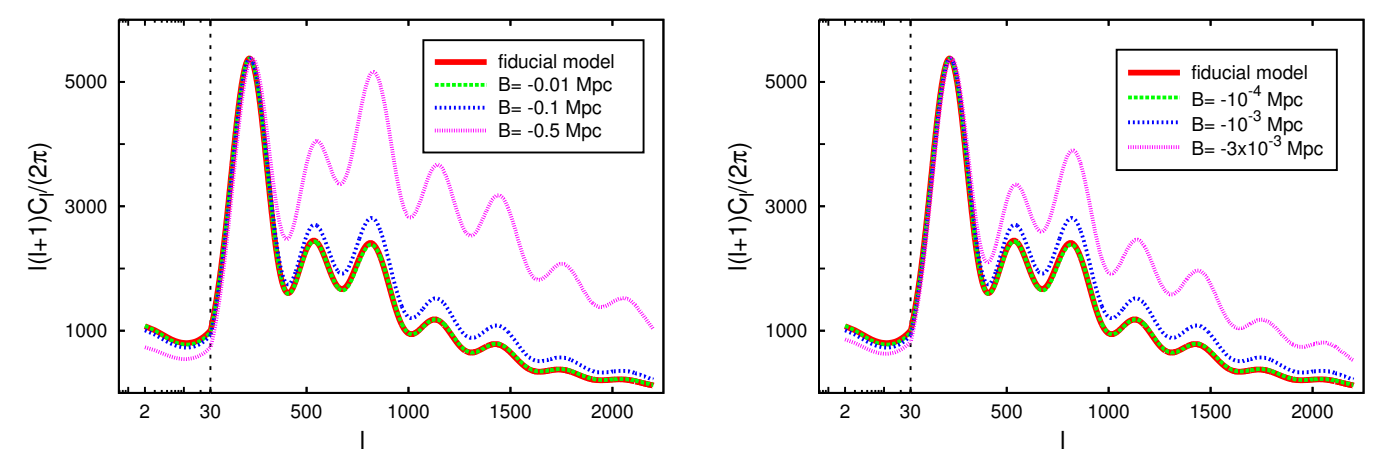

Figura 4.8. Espectro de anisotropías de la temperatura del FCR en el caso donde $k \ll \mathcal{H}\left(\eta_{\vec{k}}^{c}\right)$ para el esquema de Newton. El modelo fiducial se muestra junto con distintas predicciones para este esquema de colapso según diferentes valores para $\eta_{\vec{k}}^{c}=A / k+B$. El índice espectral escalar se fijó en $n_{s}=0.96$ mientras que $A_{s}$ toma valores tal que el máximo del primer pico coincida con el del modelo fiducial. Se muestra a la izquierda los resultados para $A=-10^{-1}$ y a la derecha $A=-10^{-3}$. León et al. (2015b).

que marca el modelo fiducial tanto para arriba como para abajo según los valores adoptados para el tiempo de colapso, lo mismo ocurre con los valles. Por eso estos esquemas resultan buenos candidatos para someter a análisis estadístico y restringir el valor de los parámetros libres con los datos observacionales. Del análisis de los gráficos, se espera que los valores de $B$ sean pequeños.

En resumen, el análisis precedente de las predicciones teóricas para las anisotropías del FCR indica que tanto para los esquemas de Newton como el de Wigner la comparación con los datos actuales pueda restringir el valor para B. Para el esquema Independiente, en cambio, cualquier valor del tiempo de colapso puede explicar los datos observacionales. 

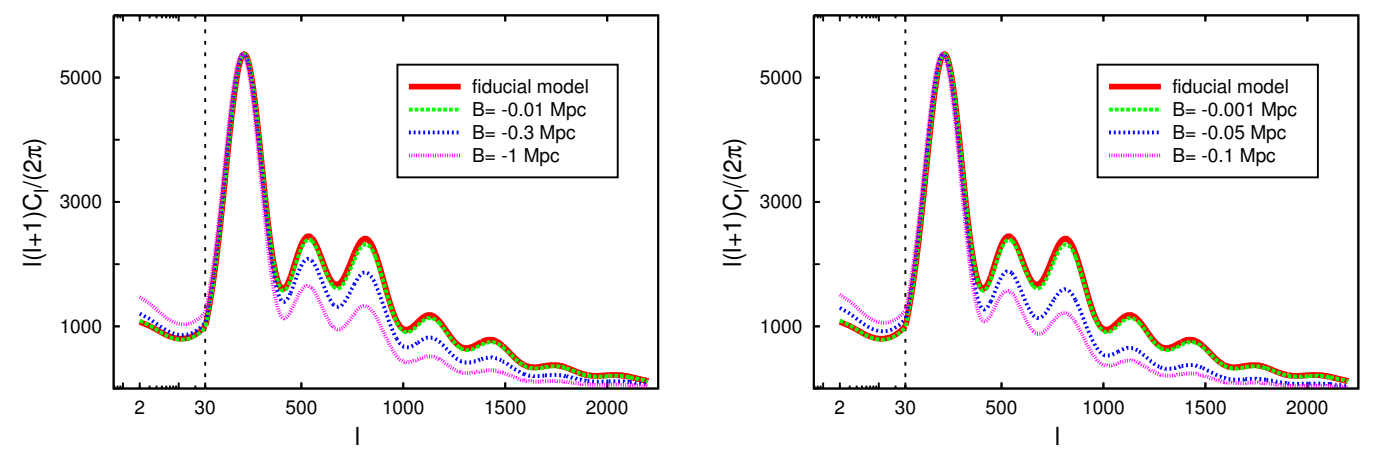

Figura 4.9. Espectro de anisotropías de la temperatura del FCR en el caso donde $k \ll \mathcal{H}\left(\eta_{\vec{k}}^{c}\right)$ para el esquema de Wigner. El modelo fiducial se muestra junto con distintas predicciones para este esquema de colapso según diferentes valores para $\eta_{\vec{k}}^{c}=A / k+B$. El índice espectral escalar se fijó en $n_{s}=0.96$ mientras que $A_{s}$ toma valores tal que el máximo del primer pico coincida con el del modelo fiducial. Se muestra a la izquierda los resultados para $A=-10^{-2}$ y a la derecha para $A=-10^{-1}$. León et al. (2015b).
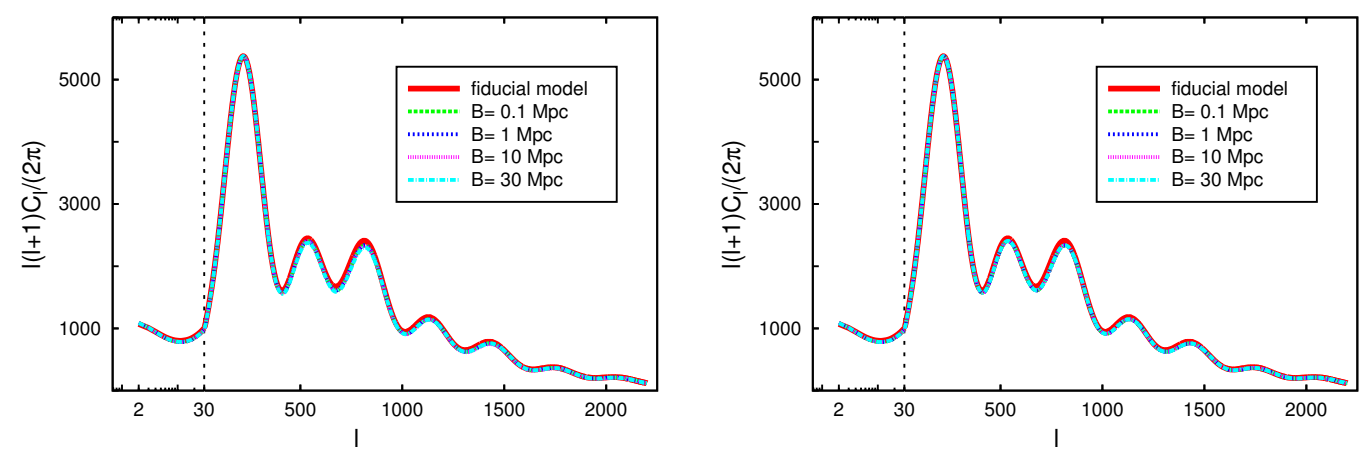

Figura 4.10. Espectro de anisotropías de la temperatura del FCR en el caso donde $k \gg$ $\mathcal{H}\left(\eta_{\vec{k}}^{c}\right)$ para el esquema Independiente. El modelo fiducial se muestra junto con distintas predicciones para este esquema de colapso según diferentes valores para $\eta_{\vec{k}}^{c}=A / k+B$. El índice espectral escalar se fijó en $n_{s}=0.96$ mientras que $A_{s}$ toma valores tal que el máximo del primer pico coincida con el del modelo fiducial. Se muestra a la izquierda los resultados para $A=-10^{2}$ y a la derecha para $A=-10^{6}$. León et al. (2015b).

\subsection{Colapso mediante el mecanismo CSL}

La implementación de la Localización Continua y Espontánea (Continuous Spontaneous Localization - CSL) a los modelos de inflación slow-roll utilizando la aproximación semiclásica fue llevada a cabo por Cañate et al. (2013). En esta sección se presentarán los avances de este mismo modelo desarrollado en una métrica cuasi-de Sitter (Piccirilli et al., 2018).

El modelo CSL se basa en proponer una modificación a la ecuación de Schrödinger, lo cual resulta en un colapso autoinducido y continuo de la función de onda del campo inflatón, hacia uno de los posibles estados propios del operador colapso $\hat{A}$ con una tasa $\lambda$. El mismo se da como parte de la evolución propia del sistema sin requerir la intervención de observador alguno, y es el resultado de la interacción del sistema con el ruido de fondo $W(t)$ que es un proceso estocástico tipo Wiener ${ }^{6}$. El parámetro $\lambda$ caracteriza la tasa con la que la función de

\footnotetext{
${ }^{6}$ En matemáticas, un proceso de Wiener es un tipo de proceso estocástico de tiempo continuo, muchas
} 

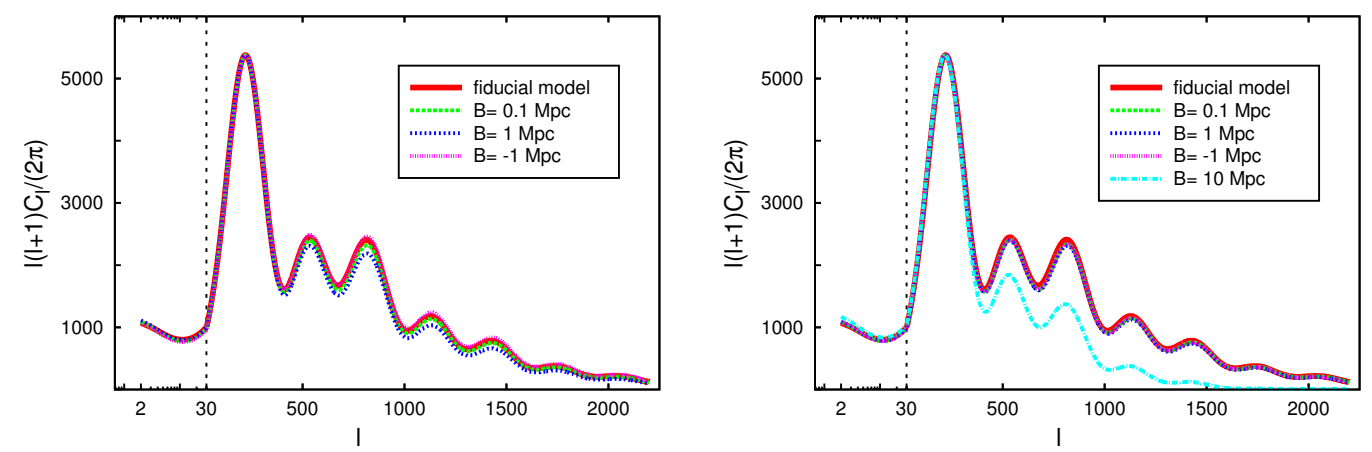

Figura 4.11. Espectro de anisotropías de la temperatura del FCR en el caso donde $k \gg \mathcal{H}\left(\eta_{\vec{k}}^{c}\right)$ para el esquema de Newton. El modelo fiducial se muestra junto con distintas predicciones para este esquema de colapso según diferentes valores para $\eta_{\vec{k}}^{c}=A / k+B$. El índice espectral escalar se fijó en $n_{s}=0.96$ mientras que $A_{s}$ toma valores tal que el máximo del primer pico coincida con el del modelo fiducial. Se muestra a la izquierda los resultados para $A=-10^{2}$ y a la derecha para $A=-10^{5}$. León et al. (2015b).
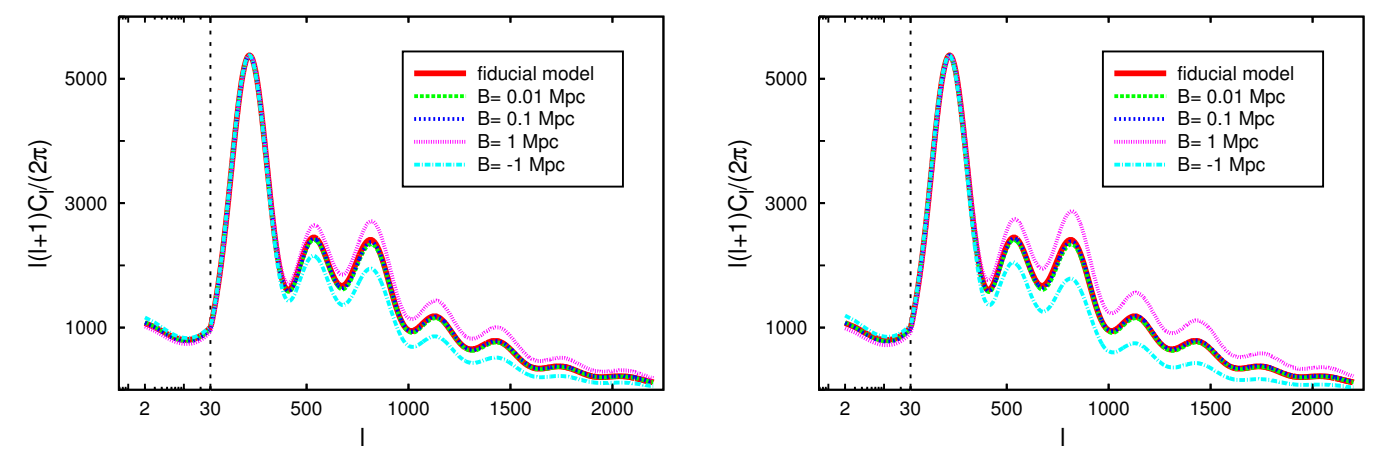

Figura 4.12. Espectro de anisotropías de la temperatura del FCR en el caso donde $k \gg \mathcal{H}\left(\eta_{\vec{k}}^{c}\right)$ para el esquema de Wigner. El modelo fiducial se muestra junto con distintas predicciones para este esquema de colapso según diferentes valores para $\eta_{\vec{k}}^{c}=A / k+B$. El índice espectral escalar se fijó en $n_{s}=0.96$ mientras que $A_{s}$ toma valores tal que el máximo del primer pico coincida con el del modelo fiducial. Se muestra a la izquierda los resultados para $A=-10^{2}$ y a la derecha para $A=-10^{6}$. León et al. (2015b).

onda incrementa su localización en la base propia del operador colapso, y es universal para el mecanismo de CSL.

La teoría está caracterizada por una ecuación de Schrödinger modificada:

$$
d|\phi\rangle=\left\{\left[-i \hat{H}-\frac{\lambda}{2}(\hat{A}-\langle\hat{A}\rangle)^{2}\right] d t+\sqrt{\lambda}(\hat{A}-\langle\hat{A}\rangle) d W_{t}\right\}|\phi\rangle
$$

que corresponde a una sola partícula y donde el comportamiento estocástico está codificado en el ruido $W(t)$ que satisface:

$$
\mathbb{E}\left(d W_{t}\right)=0, \quad \mathbb{E}\left(d W_{t} d W_{t^{\prime}}\right)=\delta\left(t-t^{\prime}\right) d t^{2},
$$

siendo $\mathbb{E}$ un promedio sobre ensambles. La función $W(t)$ es clásica y su probabilidad está veces referido también como movimiento browniano estándar. 
determinada por

$$
P D w(t) \equiv\langle\psi, t \mid \psi, t\rangle \prod_{t_{i}=0}^{t} \frac{d w\left(t_{i}\right)}{\sqrt{2 \pi \lambda / d t}},
$$

que se denomina Regla de Probabilidad. El vector de estado evoluciona de manera dinámica, de modo que aquel con mayor norma será el más probable.

La solución a la ecuación (4.107) es

$$
|\psi, t\rangle=\hat{\mathcal{T}} e^{-\int_{0}^{t} d t^{\prime}\left[i \hat{H}+\frac{1}{4 \lambda}\left[W\left(t^{\prime}\right)-2 \lambda \hat{A}\right]^{2}\right]}|\psi, 0\rangle,
$$

donde $\hat{\mathcal{T}}$ es el operador de orden temporal. No está demás recordar que la ecuación (4.110) es no relativista.

El hamiltoniano que caracteriza la inhomogeneidad del inflatón es

$$
H=(1 / 2) \int d^{3} k\left(H_{\vec{k}}^{\mathrm{R}}+H_{\vec{k}}^{\mathrm{I}}\right)
$$

donde

$$
\begin{aligned}
H_{\vec{k}}^{\mathrm{R}, \mathrm{I}} & =\pi_{\vec{k}}^{\mathrm{R}, \mathrm{I}} \pi_{\vec{k}}^{* \mathrm{R}, \mathrm{I}}+k^{2} y_{\vec{k}}^{\mathrm{R}, \mathrm{I}} y_{\vec{k}}^{* \mathrm{R}, \mathrm{I}} \\
& -\frac{\left(1-\epsilon_{1}+\epsilon_{2} / 2\right)}{\eta}\left(y_{\vec{k}}^{\mathrm{R}, \mathrm{I}} \pi_{\vec{k}}^{* \mathrm{R}, \mathrm{I}}+y_{\vec{k}}^{* \mathrm{R}, \mathrm{I}} \pi_{\vec{k}}^{\mathrm{R}, \mathrm{I}}\right),
\end{aligned}
$$

siendo $y_{\vec{k}}=a \delta \phi_{\vec{k}}$ y $\pi_{\vec{k}} \equiv y_{\vec{k}}^{\prime}-\mathcal{H} y_{\vec{k}} ; \epsilon_{1}$ y $\epsilon_{2}$ son los parámetros de slow-roll definidos al final de la sección 3.7. Los índices R,I denotan las partes reales e imaginarias de $y_{\vec{k}} \mathrm{y} \pi_{\vec{k}}$, que luego son promovidos a operadores cuánticos imponiendo las relaciones de conmutación canónica $\left[\hat{y}_{\vec{k}}^{\mathrm{R}, \mathrm{I}}, \hat{\pi}_{\vec{k}}^{\mathrm{R}, \mathrm{I}}\right]=i \delta\left(\vec{k}-\vec{k}^{\prime}\right)$.

Para la función de onda se utiliza la representación en el espacio de momento. Sea $\Phi[\pi]$ la función de onda que caracteriza el estado cuántico del campo. En el espacio de Fourier, la función de onda puede ser factorizada según las componentes de cada modo: $\Phi[\pi]=$ $\Pi_{\vec{k}} \Phi_{\vec{k}}^{\mathrm{R}}\left[\pi_{\vec{k}}^{\mathrm{R}}\right] \times \Phi_{\vec{k}}^{\mathrm{I}}\left[\pi_{\vec{k}}^{\mathrm{I}}\right]$, y se considera gaussiana durante toda la evolución. La misma resulta de la forma

$$
\Phi^{\mathrm{R}, \mathrm{I}}\left(\eta, \pi_{\vec{k}}^{\mathrm{R}, \mathrm{I}}\right)=\exp \left[-A_{k}(\eta)\left(\pi_{\vec{k}}^{\mathrm{R}, \mathrm{I}}\right)^{2}+B_{k}^{\mathrm{R}, \mathrm{I}}(\eta) \pi_{\vec{k}}^{\mathrm{R}, \mathrm{I}}+C_{k}^{\mathrm{R}, \mathrm{I}}(\eta)\right]
$$

Una identificación posible para el operador de colapso $\hat{A}$ es $\hat{\pi}_{\vec{k}}^{\mathrm{R}, \mathrm{I}}$, lo cual está motivado por el hecho de que la perturbación de la métrica $\Psi$ está directamente relacionada al valor de expectación del operador momento conjugado del campo inflatón (ver ecuación (4.16)). El mecanismo de reducción de CSL puede aplicarse independientemente sobre cada modo ${ }^{7}$, por lo que la evolución del vector estado asociado a $\Phi_{\vec{k}}^{\mathrm{R}, \mathrm{I}}\left(\eta, \pi_{\vec{k}}^{\mathrm{R}, \mathrm{I}}\right)$ es

$$
\begin{aligned}
\left|\Phi_{\vec{k}}^{\mathrm{R}, \mathrm{I}}, \eta\right\rangle & =\hat{T} \exp \left\{-\int_{\tau}^{\eta} d \eta^{\prime}\left[i \hat{H}_{\vec{k}}^{\mathrm{R}, \mathrm{I}}\right.\right. \\
& \left.\left.+\frac{1}{4 \lambda_{k}}\left(\mathcal{W}_{\vec{k}}\left(\eta^{\prime}\right)-2 \lambda_{k}^{2} \hat{\pi}_{\vec{k}}^{\mathrm{R}, \mathrm{I}}\right)^{2}\right]\right\}\left|\Phi_{\vec{k}}^{\mathrm{R}, \mathrm{I}}, \tau\right\rangle,
\end{aligned}
$$

\footnotetext{
${ }^{7}$ En el trabajo de Cañate et al. (2013) se demuestra que con la elección apropiada de los operadores de colapso del campo y teniendo en cuenta la ley de evolución de CSL, el colapso se da en los operadores correspondientes a las componentes de Fourier del campo y su momento conjugado. Con este resultado se garantiza que a primer orden en la teoría de perturbaciones cada modo colapsa independientemente.
} 
donde $\hat{T}$ es el operador de orden temporal y $\tau$ el tiempo conforme al principio de inflación. La equación (4.114) consiste en un ansatz y es la propuesta para la generalización de la ecuación (4.107) a la Teoría Cuántica de Campos (que es una teoría cuántica relativista).

La ecuación de evolución (4.114) para la ecuación de onda (4.113) determina un conjunto de ecuaciones dinámicas para $A_{k}(\eta), B_{k}^{\mathrm{R}, \mathrm{I}}(\eta)$ y $C_{k}^{\mathrm{R}, \mathrm{I}}(\eta)$. Las condiciones iniciales son determinadas por el vacío de Bunch-Davies, que es el estado inicial del campo, con lo que se obtiene: $A_{k}(\tau)=1 / 2 k, B_{k}^{\mathrm{R}, \mathrm{I}}(\tau)=0$ y $C_{k}^{\mathrm{R}, \mathrm{I}}(\tau)=0$. El espectro primordial de potencias está directamente relacionado con la cantidad $A_{k}(\tau)$, y su estudio indica que (Cañate et al., 2013):

$$
A_{k}^{\prime}=\frac{i}{2}+\lambda_{k}-2 A_{k} \frac{\left(1-\epsilon_{1}+\epsilon_{2} / 2\right)}{\eta}-2 i k^{2} A_{k}^{2}
$$

cuya solución es

$$
A_{k}(\eta)=\frac{q}{2 i k^{2}}\left[\frac{J_{\mu+1}(-q \eta)+e^{-i \pi \mu} J_{-\mu-1}(-q \eta)}{J_{\mu}(-q \eta)-e^{-i \pi \mu} J_{-\mu}(-q \eta)}\right],
$$

siendo $q^{2} \equiv k^{2}\left(1-2 i \lambda_{k}\right)$ y $\mu \equiv 1 / 2-\epsilon_{1}+\epsilon_{2} / 2$, mientras que las $J_{\mu}$ corresponden a las funciones de Bessel.

\subsubsection{Espectro primordial de potencias}

La deducción del espectro de potencias es análogo al análisis realizado en la sección 4.4.4 y luego se identifica por comparación directa con la expresión correspondiente a los coeficientes de las anisotropías angulares del FCR, un espectro de potencias efectivo y sin dimensiones:

$$
P(k)=\frac{H^{2}}{k M_{P}^{2} \epsilon_{1}} \overline{\left\langle\hat{\pi}_{\vec{k}}\right\rangle\left\langle\hat{\pi}_{\vec{k}}\right\rangle^{*}} .
$$

El promedio $\overline{\left\langle\hat{\pi}_{\vec{k}}\right\rangle\left\langle\hat{\pi}_{\vec{k}}\right\rangle^{*}}$ se puede reescribir de la siguiente manera:

$$
\begin{aligned}
\overline{\left\langle\hat{\pi}_{\vec{k}}\right\rangle\left\langle\hat{\pi}_{\vec{k}}\right\rangle^{*}} & =\overline{\left\langle\hat{\pi}_{\vec{k}}^{\mathrm{R}}+i \hat{\pi}_{\vec{k}}^{\mathrm{I}}\right\rangle\left\langle\hat{\pi}_{\vec{k}}^{\mathrm{R}}-i \hat{\pi}_{\vec{k}}^{\mathrm{I}}\right\rangle} \\
& =\overline{\left\langle\hat{\pi}_{\vec{k}}^{\mathrm{R}}\right\rangle^{2}}+\overline{\left\langle\hat{\pi}_{\vec{k}}^{\mathrm{I}}\right\rangle^{2}} \\
& =2 \overline{\left\langle\hat{\pi}_{\vec{k}}^{\mathrm{R}}\right\rangle^{2}} .
\end{aligned}
$$

En el marco del modelo CSL se puede demostrar que (Cañate et al., 2013)

$$
\overline{\left\langle\hat{\pi}_{\vec{k}}^{\mathrm{R}, \mathrm{I}}\right\rangle^{2}}=\overline{\left\langle\left(\hat{\pi}_{\vec{k}}^{\mathrm{R}, \mathrm{I}}\right)^{2}\right\rangle}-\frac{1}{\operatorname{Re}\left[A_{k}(\eta)\right]},
$$

donde los resultados han sido generalizados al caso cuasi-de Sitter.

Con la expresión para $A_{k}(\eta)$, eq. (4.116) y aproximando $-k \eta \ll 1$ se tiene

$$
\frac{1}{\operatorname{Re}\left[A_{k}(\eta)\right]} \simeq \frac{k 2^{2 \mu-2} \sin (\pi \mu) \Gamma^{2}(\mu)(-k \eta)^{-2 \mu+1}}{\pi \zeta_{k}^{2 \mu} \sin \left(2 \mu \theta_{k}+\pi \mu\right)},
$$

donde $\zeta_{k} e^{i \theta_{k}} \equiv \sqrt{1-2 i \lambda_{k}}$. Por otro lado, la cantidad $\overline{\left\langle\left(\hat{\pi}_{\vec{k}}^{\mathrm{R}, \mathrm{I}}\right)^{2}\right\rangle}$ puede ser aproximada para el caso $-k \eta \ll 1$ por (Cañate et al., 2013):

$$
\begin{aligned}
\overline{\left\langle\left(\hat{\pi}_{\vec{k}}^{\mathrm{R}, \mathrm{I}}\right)^{2}\right\rangle} & \simeq \frac{k}{\pi} 2^{2 \mu-2} \Gamma(\mu)^{2}(-k \eta)^{-2 \mu+1} \\
& \times\left[1+\lambda_{k} \sin \gamma_{k} \cos \gamma_{k}-\frac{\lambda_{k} k \tau}{2}\left(\frac{3}{\mu+1} \sin ^{2} \gamma_{k}+\frac{\cos ^{2} \gamma_{k}}{\mu}\right)\right]
\end{aligned}
$$


siendo $\gamma_{k} \equiv-k \tau-\mu \pi / 2-3 \pi / 4$.

Reemplazando las ecuaciones (4.120) y (4.121) en (4.119), se obtiene el espectro de potencias efectivo (4.117), que tiene la forma:

$$
P(k)=A_{s} C(k) k^{n_{s}-1} .
$$

La predicción para $n_{s}$ es diferente que para el modelo cosmológico estándar pero idéntica a la obtenida para los esquemas de colapso: $n_{s}-1=2 \epsilon_{1}-\epsilon_{2}$. Los parámetros de slow-roll $\varepsilon_{1} \equiv \varepsilon_{V}$ y $\varepsilon_{2} \equiv 4 \varepsilon_{V}-2 \delta_{v}$ están definidos en la sección 3.7.

Para el caso CSL, la función $C(k)$ se puede expresar

$$
\begin{aligned}
C(k) & \equiv 1+\lambda_{k} k|\tau|+\lambda_{k} \cos (k|\tau|) \sin (k|\tau|) \\
& -\frac{1}{\zeta_{k}^{2 n_{s}-1} \cos \left[\left(2-n_{s}\right) \theta_{k}\right]}
\end{aligned}
$$

donde $\zeta_{k} \equiv\left(1+4 \lambda_{k}^{2}\right)^{1 / 4}$ y $\theta_{k} \equiv-\frac{1}{2} \arctan \left(2 \lambda_{k}\right)$.

Proponiendo una constante $\lambda_{0}$ que representa la tasa de colapso ( $\lambda$ en la ecuación (4.107)), la parametrización $\lambda_{k}=\frac{\lambda_{0}}{k}$ da como resultado un espectro de potencias casi invariante de escala, dado que las dependencias más importantes en $k$ se encuentran en el segundo término. Se ha comprobado que los términos oscilatorios en la ecuación (4.123) no producen efectos importantes en el espectro. También se ha verificado, tanto para el caso de Sitter exacto (Villalba, 2016) como para el caso cuasi-de Sitter bajo análisis, que el espectro de anisotropías del FCR no depende del valor de $\lambda_{0}$ adoptado. Si bien los datos comológicos no son sensibles a $\lambda_{0}$, el valor obtenido por experimentos independientes de laboratorios reportan valores que son consistentes con las escalas de energía requeridas para inflación. Se considera $\lambda_{0}=\frac{1}{\tau}$ donde $\tau$ depende de dos cantidades: la escala de energía característica de inflación $V_{0}$ y el número total de e-folds de inflación $N$.

Del mismo modo que en los esquemas de colapso, se exploran pequeños apartamientos respecto a la dependencia que reproduce el modelo cosmológico estándar, por lo que se propone

$$
\lambda_{k} \equiv \lambda_{0}\left(\frac{1}{k}+\frac{\alpha}{k^{2}}\right),
$$

donde se introduce el parámetro $\alpha$. El término $\alpha / k^{2}$ está motivado en los resultados obtenidos en trabajos anteriores (Benetti et al., 2016) donde se considera una parametrización con esta dependencia en el contexto de los esquemas de colapso.

La figura 4.13 muestra el comportamiento de la función $C(k)$ para distintos valores de $\alpha$; el caso particular $C(k)=1$ reproduce los resultados del modelo cosmológico estándar. Incorporar el mecanismo CSL introduce modificaciones tanto en la amplitud como en la forma del espectro primordial de potencias a valores pequeños de $k$, cuya intensidad depende del valor de $\alpha$. Por otro lado, existe una cota física impuesta sobre los posibles valores para $\alpha$. Siendo que $\lambda_{0}$ es la tasa colapso y es, por lo tanto, positiva, y en tanto $k$ es también mayor a cero, se sigue que $\lambda_{k}$ debe ser positivo. Esto implica que $\alpha>-10^{-6}$ para los valores de $k$ que tienen relevancia observacional.

\subsubsection{Espectro de Anisotropías}

El espectro de anisotropías para la temperatura se muestra en la figura 4.14, junto con un panel inferior que indica las diferencias absolutas respecto al modelo fiducial. La variación de 


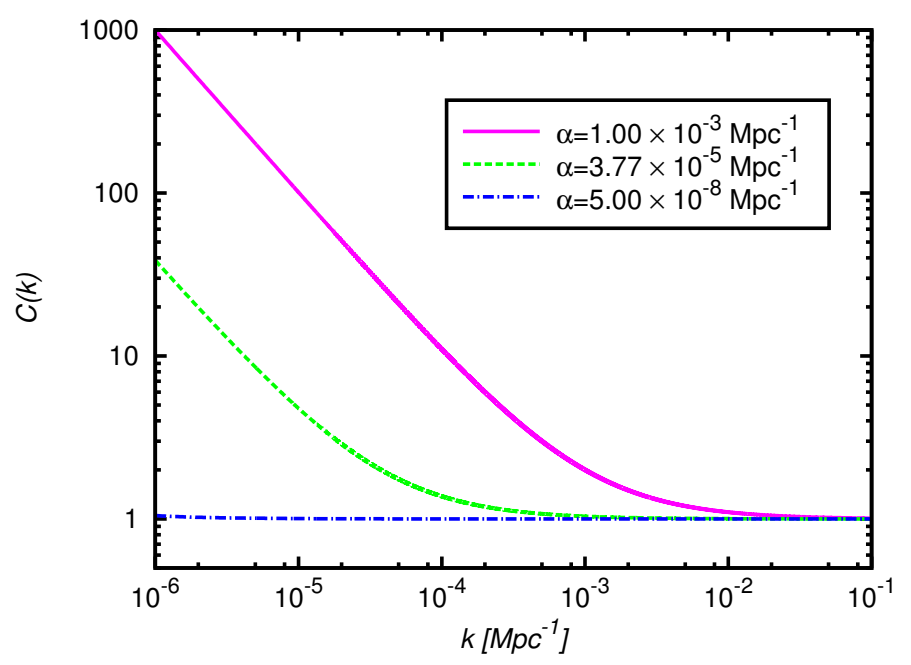

Figura 4.13. Representación de la función $C(k)$ asociada al espectro de potencias para CSL en función de los valores de $k$ relevantes para las observaciones. Se fija el valor $\lambda_{0}=$ $1 /|\tau| \simeq 6.41 \times 10^{-9} \mathrm{Mpc}^{-1}$ y $n_{s}=0.96$. Piccirilli et al. (2018).

$\alpha$ afecta principalmente a los multipolos más bajos. También se observan pequeñas diferencias de alturas en los picos para las anisotropías en la autocorrelación de los modos E (figura 4.15), mientras que el espectro de correlación cruzada de temperatura y polarización no muestra una tendencia marcada (también figura 4.15).

\subsection{Colapso durante el período de radiación}

En el capítulo 3 se explica cómo la introducción de un período inflacionario es capaz de resolver el problema del horizonte en el marco del modelo cosmológico estándar. Esto requiere considerar un período de expansión acelerada y se propone un campo escalar para describir el tipo de materia que dará lugar a inflación. La propuesta de colapso explica la transición entre un estado inicial isótropo y homogéneo a uno que ya no lo es mediante el colapso autoinducido de la función de onda del campo inflatón (Perez et al., 2006). Resulta natural proponer que este ocurre durante el período inflacionario, sin embargo no hay restricciones físicas para que esto sea así. En esta sección se considerará la posibilidad de que el colapso suceda en un tiempo propio comprendido en el período en que el Universo estuvo dominado por la radiación (León et al., 2014).

La acción en este caso debe contemplar un término correspondiente a la radiación:

$$
S=S_{r a d}+S_{G}+S_{i n f},
$$

donde $S_{G}$ y $S_{\text {inf }}$ corresponden a la acción estándar que describe la gravedad y la acción de un campo escalar $\phi$ mínimamente acoplado con la gravedad (ver sección 3.6), y $S_{\text {rad }}$ representa la acción de la componente dominante del Universo en el período de radiación.

Variar la acción (4.125) da como resultado las ecuaciones de Einstein con una contribución adicional debido a la radiación:

$$
G_{a b}=8 \pi G\left(T_{a b}^{\mathrm{rad}}+T_{a b}^{\mathrm{inf}}\right) .
$$




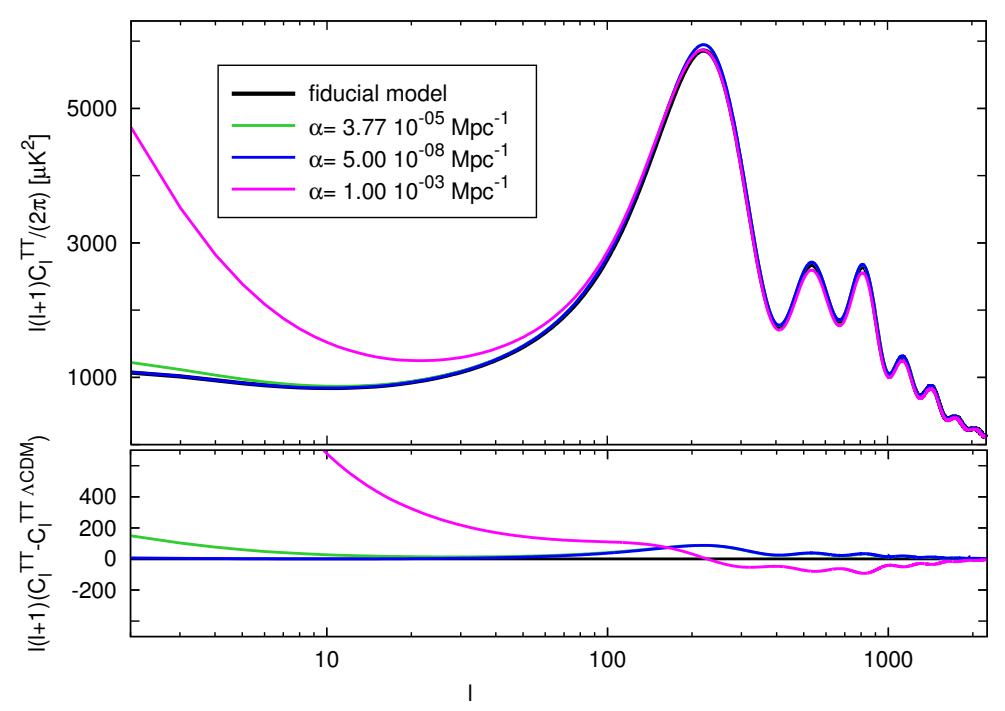

Figura 4.14. Espectro de anisotropías en temperatura para el modelo CSL (panel superior) y diferencias absolutas respecto al modelo fiducial para cada valor de $\alpha$. Cada modelo está normalizado según el máximo del primer pico del modelo fiducial. Piccirilli et al. (2018).
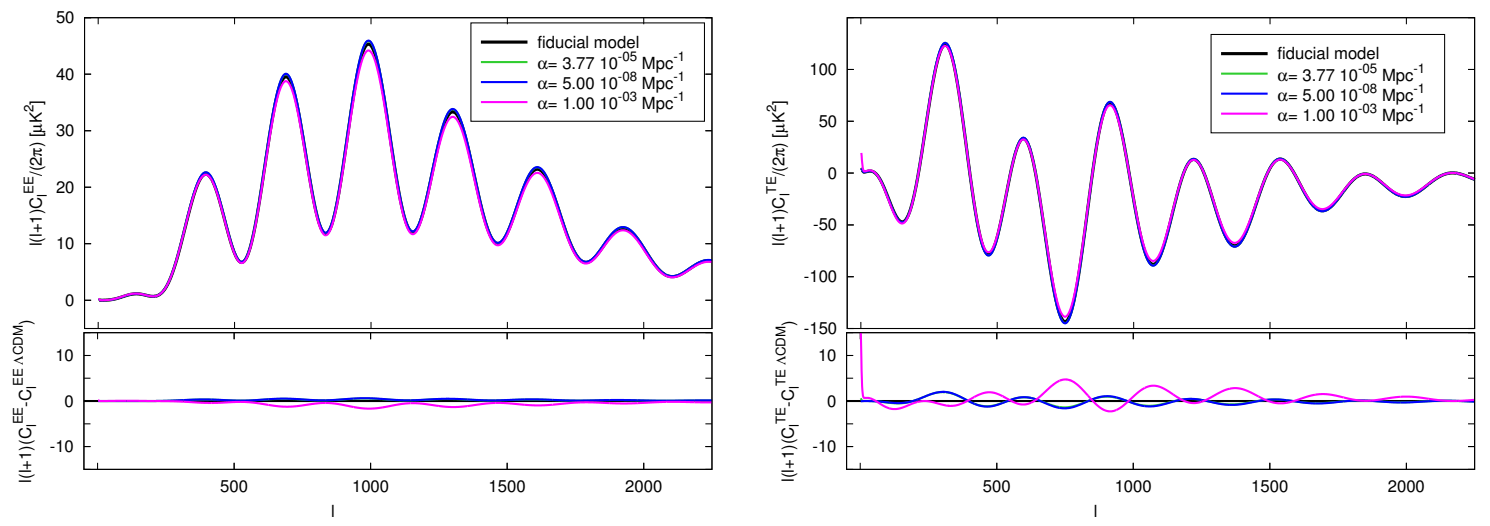

Figura 4.15. Espectro de polarización del modo E (izquierda) y correlación cruzada temperatura-modo $\mathrm{E}$ de polarización (derecha) para distintos valores de $\alpha$. Los paneles inferiores indican diferencias para cada valor del multipolo $l$ respecto a la predicción del modelo fiducial. Piccirilli et al. (2018). 
El tensor de energía-impulso para el inflatón puede escribirse

$$
T_{b}^{a}{ }^{\mathrm{inf}}=g^{a c} \nabla_{c} \phi \nabla_{b} \phi+\delta_{b}^{a}\left(\frac{1}{2} g^{c d} \nabla_{c} \phi \nabla_{d} \phi-V[\phi]\right) .
$$

Dado que la propuesta en este caso es trabajar durante el período donde el Universo está dominado por radiación, la contribución del campo inflatón debe ser despreciable: $T_{a b}^{\mathrm{inf}} \ll T_{a b}^{\mathrm{rad}}$. $\mathrm{Al}$ igual que en los casos anteriores, se separan el campo inflatón y la métrica distinguiendo el fondo (con una métrica de FRW) y las perturbaciones:

$$
\begin{aligned}
g & =g_{0}+\delta g, \\
T_{a b} & =T_{a b}^{(0)}+\delta T_{a b},
\end{aligned}
$$

luego se aplica la teoría de la perturbaciones a las ecuaciones de Einstein. Si bien predomina a orden cero la contribución del tensor de energía-impulso de radiación frente al del campo inflatón, el aporte dominante a las perturbaciones está dado por las inhomogeneidades de este último, es decir $\delta T_{a b}^{\mathrm{rad}} \ll \delta T_{a b}^{\mathrm{inf}}$.

Las ecuaciones de Einstein a primer orden dan lugar a las ecuaciones de Friedmann:

$$
G_{00}^{(0)}=8 \pi G T_{00}^{(0)}=8 \pi G a^{2} \rho,
$$

donde ahora la densidad de energía correspondiente al inflatón puede despreciarse, y se toma como ecuación de estado $P=\rho / 3$ que corresponde a la radiación. La expresión para el factor de escala es

$$
a(\eta)=C\left(\eta-\eta_{r}\right)+a_{r},
$$

siendo $\eta$ el tiempo conforme, $C$ una constante y $\eta_{r}$ es el tiempo conforme al comienzo de la era de radiación, habiendo definido $a_{r}=a\left(\eta_{r}\right)$. Normalizando el factor de escala al momento actual $a_{0}=1$ y asumiendo que inflación termina a una escala de energía de $10^{15} \mathrm{GeV}$, se encuentra que $\eta_{r} \simeq-1.2 \times 10^{-22} \mathrm{Mpc}, a_{r} \simeq 2.4 \times 10^{-28}$ y $C \simeq 1.6 \times 10^{-6} \mathrm{Mpc}^{-1}$. Para el presente tratamiento se ignorará el período de recalentamiento (reheating), por lo que el fin de inflación dará comienzo inmediato a la era de radiación: $\eta_{f i} \simeq-10^{-22} \mathrm{Mpc} \simeq \eta_{r}$.

La perturbación del espacio-tiempo puede expresarse a través del elemento de línea

$$
d s^{2}=a^{2}(\eta)\left[-(1+2 \Phi) d \eta^{2}+(1-2 \Psi) \delta_{i j} d x^{i} d x^{j}\right]
$$

donde solamente se consideran las perturbaciones escalares y se elije el gauge longitudinal para trabajar. El campo escalar puede expresarse como

$$
\phi(\vec{x}, \eta)=\phi_{0}(\eta)+\delta \phi(\vec{x}, \eta), \quad \delta \phi \ll \phi_{0} .
$$

Las ecuaciones de Einstein $\delta G_{a b}=8 \pi G \delta T_{a b}^{\text {inf }}$ a primer orden en las perturbaciones dan como resultado:

$$
\begin{gathered}
\nabla^{2} \Psi-3 \mathcal{H}\left(\mathcal{H} \Phi+\Psi^{\prime}\right)=4 \pi G\left[-\phi_{0}^{\prime 2} \Phi+\phi_{0}^{\prime} \delta \phi^{\prime}+\partial_{\phi} V a^{2} \delta \phi\right], \\
\partial_{i}\left(\mathcal{H} \Phi+\Psi^{\prime}\right)=4 \pi G \partial_{i}\left(\phi_{0}^{\prime} \delta \phi\right), \\
{\left[\Psi^{\prime \prime}+\mathcal{H}(2 \Psi+\Phi)^{\prime}+\left(2 \mathcal{H}^{\prime}+\mathcal{H}^{2}\right) \Phi+\frac{1}{2} \nabla^{2}(\Phi-\Psi)\right] \delta_{j}^{i}-\frac{1}{2} \partial^{i} \partial_{j}(\Phi-\Psi)=} \\
4 \pi G\left[\phi_{0}^{\prime} \delta \phi^{\prime}-\phi_{0}^{\prime 2} \Phi-\partial_{\phi} V a^{2} \delta \phi\right] \delta_{j}^{i} .
\end{gathered}
$$


Si sobre la última ecuación se considera $i \neq j$ y condiciones de contorno apropiadas, se obtiene la igualdad $\Psi=\Phi$, que será utilizada de aquí en más. Las primeras dos ecuaciones se combinan para obtener

$$
\nabla^{2} \Psi+4 \pi G \phi_{0}^{\prime 2} \Psi=4 \pi G\left[\phi_{0}^{\prime} \delta \phi^{\prime}+\left(a^{2} \partial_{\phi} V+3 \mathcal{H} \phi_{0}^{\prime}\right) \delta \phi\right] .
$$

Desarrollando $\Psi$ y $\phi$ en modos de Fourier, se tiene como resultado

$$
\Psi_{\vec{k}}(\eta)=\frac{4 \pi G \phi_{0}^{\prime}(\eta)}{-k^{2}+4 \pi G \phi_{0}^{\prime}(\eta)^{2}}\left[\delta \phi_{\vec{k}}^{\prime}(\eta)+\left(3 \mathcal{H}+\frac{a^{2} \partial_{\phi} V}{\phi_{0}^{\prime}(\eta)}\right) \delta \phi_{\vec{k}}(\eta)\right] .
$$

Dado que la era inflacionaria ha llegado a su fin, se cumple que $\partial_{\phi} V \simeq 0$, por lo que la densidad de energía del inflatón se puede aproximar por $\rho_{\phi} \simeq \phi_{0}^{\prime 2} / 2 a^{2} \ll \rho_{\text {rad }}$, pudiendo reescribir

$$
\Psi_{\vec{k}}(\eta)=\frac{\sqrt{\rho_{\phi}}}{\sqrt{2} M_{P}^{2}\left(-k^{2}+\rho_{\phi} a^{2} / M_{P}^{2}\right)}\left[a \delta \phi_{\vec{k}}^{\prime}(\eta)+3 \mathcal{H} a \delta \phi_{\vec{k}}(\eta)\right]
$$

que vincula las perturbaciones en el campo inflatón con las perturbaciones en la métrica. Para el caso $i=j$ en la última ecuación de (4.133) (que corresponde a las ecuaciones de Einstein con componentes $i j$ ) se puede derivar la ecuación de movimiento de las perturbaciones de la métrica:

$$
\Psi_{\vec{k}}^{\prime \prime}(\eta)+\frac{4}{\eta-\eta_{r}+a_{r} / C} \Psi_{\vec{k}}^{\prime}(\eta)+\frac{k^{2}}{3} \Psi_{\vec{k}}(\eta)=0,
$$

cuya solución analítica es

$$
\begin{aligned}
\Psi_{\vec{k}}(\eta) & =\frac{3}{\left(k \eta-\delta_{k}\right)^{2}}\left\{C_{1}(\vec{k})\left[\frac{\sqrt{3}}{k \eta-\delta_{k}} \sin \left(\frac{k \eta-\delta_{k}}{\sqrt{3}}\right)-\cos \left(\frac{k \eta-\delta_{k}}{\sqrt{3}}\right)\right]\right. \\
& \left.+C_{2}(\vec{k})\left[\frac{\sqrt{3}}{k \eta-\delta_{k}} \cos \left(\frac{k \eta-\delta_{k}}{\sqrt{3}}\right)+\sin \left(\frac{k \eta-\delta_{k}}{\sqrt{3}}\right)\right]\right\},
\end{aligned}
$$

siendo $\delta_{k} \equiv k \eta_{r}-k a_{r} / C$.

Una vez que el colapso ha tenido lugar se pueden separar, al igual que en el análisis anterior, los modos $\Psi_{k}$ en aquellos cuya longitud de onda propia asociada es mayor o menor que el radio de Hubble comóvil. En este último caso la condición está dada por $k \gg a H=\mathcal{H}$, y su expresión para la era dominada por radiación es $\mathcal{H} \equiv a^{\prime}(\eta) / a(\eta)=1 /\left(\eta-\eta_{r}+a_{r} / C\right)$. Esta condición es equivalente a $\left(k \eta-\delta_{k}\right) \gg 1$ (que se transforma en $\left(k \eta-\delta_{k}\right) \ll 1$ para los modos "fuera" del horizonte).

Si $\left(k \eta-\delta_{k}\right) \gg 1$ ocurre que $\Psi_{\vec{k}} \rightarrow 0$, por lo que no contribuyen al espectro de anisotropías. Para el caso contrario, la aproximación $\left(k \eta-\delta_{k}\right) \ll 1$ y teniendo en cuenta que el Universo se expande:

$$
\Psi_{\vec{k}}(\eta) \simeq \frac{C_{1}(\vec{k})}{3} .
$$

Es decir que las perturbaciones en la métrica son cero previas al colapso y luego son constantes. El valor de la constante $C_{1}$ se obtiene de evaluar (4.136) al tiempo de colapso $\eta_{\vec{k}}^{c}$.

\subsubsection{Análisis de las perturbaciones cuánticas}

La variable cuántica fundamental será la fluctuación del campo inflatón, por lo que el procedimiento es similar al de la sección 4.4.1, sólo que el factor de escala corresponderá al 
de la era de radiación y $\partial_{\phi \phi}^{2} V \simeq 0$ dado que la era inflacionaria ha llegado a su fin. Bajo estas consideraciones, la ecuación de movimiento resulta

$$
y_{k}^{\prime \prime}+k^{2} y_{k}=0
$$

que corresponde a un oscilador armónico. Sus soluciones son:

$$
\begin{aligned}
& y_{k}(\eta)=A_{k} e^{i k \eta}+B_{k} e^{-i k \eta} \\
& g_{k}(\eta)=-A_{k} k\left(\frac{\mathcal{H}}{k}-i\right) e^{i k \eta}-B_{k} k\left(\frac{\mathcal{H}}{k}+i\right) e^{i k \eta}
\end{aligned}
$$

donde las constantes están fijadas por las relaciones de conmutación canónica y la elección de un estado de vacío para el campo inflatón. La elección será nuevamente el vacío de BunchDavies:

$$
y_{k}(\eta)=\frac{1}{\sqrt{2 k}}\left(1-\frac{i}{k \eta}\right) e^{-i k \eta}, \quad g_{k}(\eta)=-i \sqrt{\frac{k}{2}} e^{-i k \eta} .
$$

Las constantes $A_{k}$ y $B_{k}$ son fijadas haciendo coincidir los modos durante inflación (4.142) y los modos durante radiación (4.141) al tiempo conforme $\eta_{r}$ que corresponde al inicio de la era de radiación y es de igual orden de magnitud que el tiempo conforme que marca el fin de inflación (recordar que se está ignorando la etapa intermedia de reheating). Sin embargo, no hay hasta el momento ningún mecanismo que modifique la isotropía y homogeneidad del estado vacío, por lo cual se prosigue a describir en concreto la propuesta de colapso que dará lugar a las perturbaciones primordiales en la curvatura.

\subsubsection{Perturbaciones en la curvatura}

Una vez sucedido el colapso y luego de un desarrollo teórico adecuado se espera encontrar cantidades observables, por lo que se trabajará con operadores hermíticos en analogía con la mecánica cuántica habitual. Los operadores del campo y su momento conjugado se separan en su parte real e imaginaria (ecuaciones (4.26) y (4.27)). Las reglas de conmutación no son las usuales, y vienen dadas por (4.28).

En el estado de vacío $\hat{y}_{\vec{k}}$ y $\hat{\pi}_{\vec{k}}$ responden a una distribución gaussiana centrada en cero y con incerteza $\left(\Delta \hat{y}_{\vec{k}}\right)_{0}^{2} \mathrm{y}\left(\Delta \hat{\pi}_{\vec{k}}\right)_{0}^{2}$. Los valores medios del estado post-colapso se pueden expresar:

$$
\begin{gathered}
\left\langle\hat{y}_{\vec{k}}^{R, I}\left(\eta_{\vec{k}}^{c}\right)\right\rangle_{\Theta}=\lambda_{1} x_{\vec{k}, 1}^{R, I} \sqrt{\left(\Delta \hat{y}_{\vec{k}}^{R, I}\left(\eta_{k}^{c}\right)\right)_{0}^{2}}=\lambda_{1} x_{\vec{k}, 1}^{R, I} \frac{L^{3 / 2}}{\sqrt{2}}\left|y_{k}\left(\eta_{\vec{k}}^{c}\right)\right|, \\
\left\langle\hat{\pi}_{\vec{k}}^{R, I}\left(\eta_{k}^{c}\right)\right\rangle_{\Theta}=\lambda_{2} x_{\vec{k}, 2}^{R, I} \sqrt{\left(\Delta \hat{\pi}_{\vec{k}}^{R, I}\left(\eta_{k}^{c}\right)\right)_{0}^{2}}=\lambda_{2} x_{\vec{k}, 2}^{R, I} \frac{L^{3 / 2}}{\sqrt{2}}\left|g_{k}\left(\eta_{\vec{k}}^{c}\right)\right| .
\end{gathered}
$$

Las variables $x_{\vec{k}, 1}^{R, I}, x_{\vec{k}, 2}^{R, I}$ aleatorias y su distribución corresponde a una gaussiana centrada en cero y normalizada, $\eta_{\vec{k}}^{c}$ es el tiempo de colapso y el subíndice $\Theta$ denota que el valor de expectación se toma en el estado post-colapso. Cada esquema de colapso está definido por un valor particular de $\lambda_{1}, \lambda_{2}$, que pueden adoptar los valores 0 o 1 . Por ejemplo, si el operador momento conjugado del campo inflatón es afectado por el colapso pero el operador de campo no, se configura con $\lambda_{2}=1, \lambda_{1}=0$. 


\subsubsection{Cantidades observables}

Luego del colapso, la ecuación para las perturbaciones del campo inflatón se puede expresar:

$$
\Psi_{\vec{k}}\left(\eta_{\vec{k}}^{c}\right)=\frac{\sqrt{\rho_{\phi}}}{\sqrt{2} M_{P}^{2}\left(-k^{2}+\rho_{\phi} a_{c}^{2} / M_{P}^{2}\right)}\left(\left\langle\hat{\pi}_{\vec{k}}\left(\eta_{\vec{k}}^{c}\right)\right\rangle+3 \mathcal{H}_{c}\left\langle\hat{y}_{\vec{k}}\left(\eta_{\vec{k}}^{c}\right)\right\rangle\right)
$$

donde $a_{c} \equiv a\left(\eta_{\vec{k}}^{c}\right)$ y $\mathcal{H}_{c} \equiv \mathcal{H}\left(\eta_{\vec{k}}^{c}\right)$.

Los coeficientes $a_{l m}$ vienen dados por

$$
a_{l m}=\frac{4 \pi i^{l}}{L^{3}} \sum_{\vec{k}} j_{l}\left(k R_{D}\right) Y_{l m}^{\star}(\hat{k}) T(k) \Psi_{\vec{k}}\left(\eta_{\vec{k}}^{c}\right),
$$

donde $\Psi_{\vec{k}}\left(\eta_{\vec{k}}^{c}\right)$ corresponde a los modos tal que $z_{k} \ll 1$, ya que que el resto decae a cero y no son relevantes. Generalizando para los distintos esquemas posibles de colapso:

$$
a_{l m}=\frac{2 \pi i^{l}}{L^{3 / 2}} \frac{\sqrt{\rho_{\phi}}}{M_{P}^{2}} \sum_{\vec{k}} \frac{j_{l}\left(k R_{D}\right) Y_{l m}^{\star}(\hat{k}) T(k)}{\left(-k^{2}+\rho_{\phi} a_{c}^{2} / M_{P}^{2}\right)}\left(\lambda_{2} X_{\vec{k}, 2}\left|g_{k}\left(\eta_{\vec{k}}^{c}\right)\right|+3 \mathcal{H}_{c} \lambda_{1} X_{\vec{k}, 1}\left|y_{k}\left(\eta_{\vec{k}}^{c}\right)\right|\right),
$$

donde $X_{\vec{k}, i} \equiv x_{\vec{k}, j}^{R}+i x_{\vec{k}, j}^{I}(j=1,2)$.

Para obtener una predicción susceptible de contrastarse con los datos, se utilizan las propiedades estadísticas de una caminata aleatoria en el plano complejo y se obtiene una estimación de $\left|a_{l m}\right|$ a través de su valor más probable. Se hace la identificación $\left|a_{l m}\right|_{\text {M.L. }}^{2}=$ $\overline{\left|a_{l m}\right|^{2}}$, donde la línea superior indica el promedio en un ensamble de posibles realizaciones. Aplicando estos resultados y pasando de una caja de lado L al continuo se obtiene:

$$
\left|a_{l m}\right|_{\text {M.L. }}^{2}=\frac{\rho_{\phi}}{\pi M_{P}^{4}} \int d^{3} k \frac{j_{l}\left(k R_{D}\right)^{2}\left|Y_{l m}(\hat{k})\right|^{2} T(k)^{2}}{\left(-k^{2}+\rho_{\phi} a_{c}^{2} / M_{P}^{2}\right)^{2}}\left(\lambda_{2}^{2}\left|g_{k}\left(\eta_{\vec{k}}^{c}\right)\right|^{2}+9 \mathcal{H}_{c}^{2} \lambda_{1}^{2}\left|y_{k}\left(\eta_{\vec{k}}^{c}\right)\right|^{2}\right) .
$$

La estimación para los coeficientes de las anisotropías será:

$$
\begin{aligned}
C_{l}^{\text {M.L. }} & \equiv \frac{1}{2 l+1} \sum_{m}\left|a_{l m}\right|_{\text {M.L. }}^{2} \\
& =\frac{\rho_{\phi}}{\pi M_{P}^{4}} \int_{0}^{\infty} \frac{d k}{k} \frac{j_{l}\left(k R_{D}\right)^{2} T(k)^{2} k^{3}}{\left(-k^{2}+\rho_{\phi} a_{c}^{2} / M_{P}^{2}\right)^{2}}\left(\lambda_{2}^{2}\left|g_{k}\left(\eta_{\vec{k}}^{c}\right)\right|^{2}+9 \mathcal{H}_{c}^{2} \lambda_{1}^{2}\left|y_{k}\left(\eta_{\vec{k}}^{c}\right)\right|^{2}\right) .
\end{aligned}
$$

Comparando esta expresión con la correspondiente del modelo cosmológico estándar se obtiene el espectro de potencias equivalente para el modelo de colapso. Si se asume que $\eta_{\vec{k}}^{c} \gg\left|\eta_{r}\right|$, entonces el factor de escala al momento del colapso puede aproximarse por $a_{c} \simeq C \eta_{\vec{k}}^{c}$; lo mismo ocurre con $\mathcal{H}_{c}=\left(\eta_{\vec{k}}^{c}-\eta_{r}+a_{r} / C\right)^{-1}$, que puede aproximarse por $\mathcal{H}_{c} \simeq 1 / \eta_{\vec{k}}^{c}$. Entonces, el espectro de potencias se escribe:

$$
\Delta^{2}(k) \simeq \frac{9 \rho_{\phi}}{8 \pi^{2} M_{P}^{4}} \frac{k^{4}}{\left[-k^{2}+\rho_{\phi}\left(C \eta_{\vec{k}}^{c} / M_{P}\right)^{2}\right]^{2}}\left(\lambda_{2}^{2} N\left(z_{k}\right)+9 \lambda_{1}^{2} M\left(z_{k}\right)\right) .
$$

Considerando $\sigma_{k} \equiv k \eta_{r} \ll 1$, las expresiones para $M\left(z_{k}\right)$ y $N\left(z_{k}\right)$ resultan a orden $\mathcal{O}\left(\sigma_{k}^{-4}\right)$

$$
\begin{aligned}
M\left(z_{k}\right) & \simeq \frac{1}{\sigma_{k}^{4}} \frac{\sin ^{2} z_{k}}{z_{k}^{2}} \\
N\left(z_{k}\right) & \simeq \frac{1}{\sigma_{k}^{4}}\left[\frac{1}{2}+\frac{1}{2 z_{k}^{2}}+\cos \left(2 z_{k}\right)\left(\frac{1}{2}-\frac{1}{2 z_{k}^{2}}\right)-\frac{\sin \left(2 z_{k}\right)}{z_{k}}\right] .
\end{aligned}
$$




\subsubsection{Análisis del espectro de potencias}

Debido a que el desarrollo para este modelo fue realizado con una métrica a orden cero en un espacio-tiempo de Sitter exacto para describir el período inflacionario, al comparar con el espectro de potencias del modelo cosmológico estándar $\Delta^{2}(k)=\mathcal{A} k^{n_{s}-1}$ se fija $n_{s}=1$. Esto da lugar a un espectro invariante de escala, lo cual está descartado a más de $5 \sigma$ por las últimas observaciones del FCR. Sin embargo, el análisis consistirá en evaluar los distintos esquemas del modelo y ver si pueden recuperar un espectro invariante de escala (que sería su correspondiente en el modelo estándar, con $n_{s}=1$ ).

$\mathrm{Al}$ igual que en trabajos anteriores donde se utilizó la aproximación de un espacio-tiempo de de Sitter exacto para el período inflacionario (Landau, Scóccola, \& Sudarsky, 2012), se observa que el resultado final no es exactamente un espectro de potencias invariante de escala, ya que el colapso introduce términos dependientes de $k$ logrando un pequeño apartamiento que resulta consistente con las observaciones.

De la definición del espectro de potencias (4.149) pueden tomarse dos nuevos límites: $k^{2} \ll \rho_{\phi}\left(C \eta_{\vec{k}}^{c} / M_{P}\right)^{2}, k^{2} \gg \rho_{\phi}\left(C \eta_{\vec{k}}^{c} / M_{P}\right)^{2}$. Además se estudian los distintos esquemas que están determinados por el valor de $\lambda_{1}$ y $\lambda_{2}$. Las distintas posibilidades se resumen en la tabla 4.1 .

\begin{tabular}{lcccc}
\hline caso & límite & $\lambda_{\mathbf{1}}$ & $\lambda_{\mathbf{2}}$ & viabilidad \\
\hline $\mathrm{A}$ & $k^{2} \ll \rho_{\phi}\left(C \eta_{\vec{k}}^{c} / M_{P}\right)^{2}$ & 0 & 1 & No \\
$\mathrm{B}$ & $k^{2} \ll \rho_{\phi}\left(C \eta_{\vec{k}}^{c} / M_{P}\right)^{2}$ & 1 & 0 & Sí \\
$\mathrm{C}$ & $k^{2} \ll \rho_{\phi}\left(C \eta_{\vec{k}}^{c} / M_{P}\right)^{2}$ & 1 & 1 & Sí \\
$\mathrm{D}$ & $k^{2} \gg \rho_{\phi}\left(C \eta_{\vec{k}}^{c} / M_{P}\right)^{2}$ & 1 & 0 & No \\
$\mathrm{E}$ & $k^{2} \gg \rho_{\phi}\left(C \eta_{\vec{k}}^{c} / M_{P}\right)^{2}$ & 0 & 1 & No \\
$\mathrm{F}$ & $k^{2} \gg \rho_{\phi}\left(C \eta_{\vec{k}}^{c} / M_{P}\right)^{2}$ & 1 & 1 & No \\
\hline
\end{tabular}

Tabla 4.1. Análisis de los diferentes casos posibles para el colapso durante la época de radiación. $\lambda_{1}$ y $\lambda_{2}$ indican una forma de generalizar los esquemas propuestos del colapso de la función de onda y momento conjugado del campo inflatón.

Para los casos A, B y C se tiene en cuenta la condición $k^{2} \ll \rho_{\phi}\left(C \eta_{\vec{k}}^{c} / M_{P}\right)^{2}$, para lo cual el espectro de potencias (4.149) se puede aproximar por

$$
\Delta^{2}(k) \simeq \frac{9}{8 \pi^{2}} \frac{k^{4}}{\rho_{\phi}\left(C \eta_{\vec{k}}^{c}\right)^{4}}\left[1+2 \beta_{k}\right]\left[\lambda_{2}^{2} N\left(z_{k}\right)+9 \lambda_{1}^{2} M\left(z_{k}\right)\right],
$$

donde se define

$$
\beta_{k} \equiv \frac{k^{2} M_{P}^{2}}{\rho_{\phi}\left(C \eta_{\vec{k}}^{c}\right)^{2}},
$$

y $M\left(z_{k}\right), N\left(z_{k}\right)$ están dados por las ecuaciones (4.150). La condición $k^{2} \ll \rho_{\phi}\left(C \eta_{\vec{k}}^{c} / M_{P}\right)^{2}$ implica $\beta \ll 1$.

Para asegurar que la longitud de onda propia del modo sea más grande que el radio de Hubble al momento del colapso se debe cumplir $z_{k} \ll 1$, lo cual permite expandir en series 
$M\left(z_{k}\right), N\left(z_{k}\right)$ :

$$
M\left(z_{k}\right) \simeq \frac{1}{\sigma_{k}^{4}}\left(1-\frac{z_{k}^{2}}{3}\right), \quad N\left(z_{k}\right) \simeq \frac{1}{\sigma_{k}^{4}} \frac{z_{k}^{4}}{9} .
$$

Caso A Tomando los valores $\lambda_{1}=0$ and $\lambda_{2}=1$ la variable correspondiente al momento conjugado del campo modifica su valor de expectación luego del colapso pero no así la variable del campo. El espectro primordial de potencias resulta:

$$
\Delta^{2}(k) \simeq \frac{1}{8 \pi^{2}} \frac{1}{\rho_{\phi}\left(\eta_{r} C\right)^{4}}\left[1+2 \beta_{k}\right] k^{4},
$$

donde se hizo la sustitución $z_{k} \equiv k \eta_{\vec{k}}^{c}$.

El mismo lleva la dependencia $k^{4}$ y el término dominante no cuenta con parámetros tales permitan recuperar el espectro invariante de escala imponiendo alguna condición. Por ende, este esquema no resulta viable ya que no puede recuperar la predicción para el espectro de potencias del modelo cosmológico estándar en un espacio-tiempo de Sitter.

Caso B La configuración en esta oportunidad es $\lambda_{1}=1, \lambda_{2}=0$, que da lugar a:

$$
\Delta^{2}(k) \simeq \frac{81}{8 \pi^{2}} \frac{1}{\rho_{\phi}\left(\eta_{r} C \eta_{\vec{k}}^{c}\right)^{4}}\left[1+k^{2}\left(\frac{2 M_{P}^{2}}{\rho_{\phi} C^{2} \eta_{\vec{k}}^{c 2}}-\frac{\eta_{\vec{k}}^{c 2}}{3}\right)\right] .
$$

Entonces si $\eta_{\vec{k}}^{c}$ es independiente de $k$, se recupera un espectro de potencias plano más pequeñas correcciones de la forma $k^{2}$.

El siguiente paso es hacer algunas pruebas de consistencia. Por ejemplo, se busca que la amplitud del espectro de potencias coincida al menos en orden de magnitud con el que determinan las observaciones sobre el modelo cosmológico estándar:

$$
\frac{81}{8 \pi^{2}} \frac{1}{\rho_{\phi}\left(\eta_{r} C \eta_{\vec{k}}^{c}\right)^{4}} \simeq 10^{-9} .
$$

Con los valores numéricos para $C$ y $\eta_{r}$ se obtiene:

$$
\rho_{\phi}^{-1} \simeq 10^{-120} \eta_{\vec{k}}^{c 4} \text {. }
$$

De la condición $\beta_{k} \ll 1$

$$
\frac{k^{2} M_{P}^{2}}{\rho_{\phi}\left(C \eta_{\vec{k}}^{c}\right)^{2}} \ll 1
$$

y teniendo en cuenta además que $k \simeq 10^{-1} \mathrm{Mpc}^{-1}$ es el mayor de los valores de $k$ entre los que resultan relevantes para las observaciones, es posible establecer un límite superior para el tiempo de colapso:

$$
\eta_{\vec{k}}^{c} \ll 10^{-2} \mathrm{Mpc} .
$$

Esto implica que el colapso debe tener lugar mucho antes que la época de nucleosíntesis. De la ecuación de Friedmann se puede estimar la densidad de energía de radiación:

$$
\rho_{\mathrm{rad}}=\frac{3 M_{P}^{2} \mathcal{H}_{c}^{2}}{a_{c}^{2}} \simeq \frac{3 M_{P}^{2}}{C^{2} \eta_{\vec{k}}^{c 4}} \simeq \frac{3 M_{P}^{2} 10^{-120} \rho_{\phi}}{C^{2}}
$$

que junto con (4.157) y los valores numéricos de $C$ y $M_{p l}$ da como resultado

$$
\rho_{\phi} \simeq 10^{-5} \rho_{\mathrm{rad}}
$$

lo cual es consistente con el requerimiento $\rho_{\mathrm{rad}} \gg \rho_{\phi} \mathrm{y}$, por lo tanto, este esquema resulta viable y susceptible de compararse con las observaciones. 
Caso C Se asignan ahora los valores $\lambda_{1}=\lambda_{2}=1 \mathrm{y}$ el espectro de potencias puede ser aproximado por:

$$
\Delta^{2}(k) \simeq \frac{81}{8 \pi^{2}} \frac{k^{4}}{\rho_{\phi}\left(C \eta_{\vec{k}}^{c}\right)^{4}}\left[1+2 \beta_{k}\right] \frac{1}{\sigma_{k}^{4}}\left[1-\frac{z_{k}^{2}}{3}+\frac{z_{k}^{4}}{81}\right] .
$$

El término dominante es de la misma forma que el caso B, por lo que el análisis es similar y el esquema resulta viable.

Los casos D, E y F tienen en cuenta la aproximación $k^{2} \gg \rho_{\phi}\left(C \eta_{\vec{k}}^{c} / M_{P}\right)^{2}$, lo cual implica $\beta_{k} \gg 1$. El espectro de potencias de la ecuación (4.149) se puede escribir:

$$
\Delta^{2}(k) \simeq \frac{9 \rho_{\phi}}{8 \pi^{2} M_{P}^{4}}\left(1+\frac{2}{\beta_{k}}\right)\left(\lambda_{2}^{2} N\left(z_{k}\right)+9 \lambda_{1}^{2} M\left(z_{k}\right)\right) .
$$

Caso D En esta ocasión se consideran los valores $\lambda_{1}=1$ and $\lambda_{2}=0$, por lo que el espectro primordial de potencias toma la forma

$$
\Delta^{2}(k) \simeq \frac{81 \rho_{\phi}}{8 \pi^{2} M_{P}^{4} \eta_{r}^{4}}\left(1+\frac{2}{\beta_{k}}\right) k^{-4}\left(1-\frac{z_{k}^{2}}{3}\right) .
$$

El término dominante es proporcional a $k^{-4}$ y no depende del tiempo de colapso, por lo que no se recupera el espectro estándar y debe ser descartado.

Caso E Si $\lambda_{1}=0$ and $\lambda_{2}=1$ se obtiene

$$
\Delta^{2}(k) \simeq \frac{9 \rho_{\phi}}{8 \pi^{2} M_{P}^{4}}\left(1+\frac{2}{\beta_{k}}\right) \frac{1}{\sigma_{k}^{4}} \frac{z_{k}^{4}}{9} .
$$

Sustituyendo $\beta_{k}$ and $z_{k}$ :

$$
\Delta^{2}(k) \simeq \frac{\rho_{\phi}}{8 \pi^{2} M_{P}^{4}} \frac{\eta_{\vec{k}}^{c 4}}{\eta_{r}^{4}}\left(1+\frac{2 \rho_{\phi}\left(C \eta_{\vec{k}}^{c}\right)^{2}}{k^{2} M_{P}^{2}}\right) .
$$

Proponiendo para este caso una parametrización del tiempo de colapso independiente de $k$, el esquema predice un espectro de potencias invariante de escala más correcciones de la forma $k^{-2}$.

Se proceden a realizar algunos chequeos de consistencia, como por ejemplo el de la amplitud correspondiente al modelo cosmológico estándar según las observaciones. Se busca que coincidan al menos en orden de magnitud:

$$
\frac{\rho_{\phi}}{8 \pi^{2} M_{P}^{4}} \frac{\eta_{\vec{k}}^{c 4}}{\eta_{r}^{4}} \simeq 10^{-9}
$$

que da lugar a la siguiente relación entre la densidad de energía y el tiempo de colapso:

$$
\rho_{\phi}^{-1} \simeq 10^{-129} \eta_{\vec{k}}^{c 4} \text {. }
$$

La condición $\beta_{k} \gg 1$ se escribe

$$
\frac{k^{2} M_{P}^{2}}{\rho_{\phi}\left(C \eta_{\vec{k}}^{c}\right)^{2}} \gg 1
$$


Combinando estas ecuaciones y asignando los valores numéricos correspondientes, para el valor más pequeño de los modos que resulta de interés observacional $k \simeq 10^{-6} \mathrm{Mpc}^{-1}$, se tiene que

$$
\eta_{\vec{k}}^{c} \gg 10^{8} \mathrm{Mpc},
$$

que resulta 6 órdenes de magnitud mayor al tiempo de desacople, razón por la cual este esquema debe ser descartado.

Caso $\mathbf{F}$ La configuración para este esquema es $\lambda_{1}=\lambda_{2}=1$, y el espectro de potencias se escribe:

$$
\Delta^{2}(k) \simeq \frac{81 \rho_{\phi}}{8 \pi^{2} M_{P}^{4} \eta_{r}^{4}}\left(1+\frac{2}{\beta_{k}}\right)\left(1-\frac{z_{k}^{2}}{3}+\frac{z_{k}^{4}}{81}\right) k^{-4} .
$$

El término dominante es de la forma $k^{-4}$, por lo que este esquema se descarta.

En resumen, las condiciones a partir de las cuales se puede reproducir un espectro invariante de escala son: i) la variable correspondiente al campo inflatón debe modificar su valor de expectación luego del colapso (es decir $\left\langle\hat{y}_{\vec{k}}\left(\eta_{\vec{k}}^{c}\right)\right\rangle \neq 0$ ), ii) el tiempo de colapso debe ser independiente de $k$ (o sea $\eta_{\vec{k}}^{c}=\eta_{c}$ ), y iii) satisfacer la condición $\eta_{\vec{k}}^{c} \ll 10^{-2} \mathrm{Mpc}$. Con estas condiciones, el espectro de potencias se puede escribir:

$$
\Delta^{2}(k) \simeq \mathcal{A} C(k)
$$

donde

$$
\begin{gathered}
\mathcal{A} \equiv \frac{81}{8 \pi^{2}} \frac{1}{\rho_{\phi} C^{4} \eta_{r}^{4} \eta_{c}{ }^{4}}, \\
C(k) \equiv\left(1+2 \beta_{k}\right)\left\{\frac{\sin ^{2}\left(k \eta_{c}\right)}{\left(k \eta_{c}\right)^{2}}+\frac{\lambda_{2}^{2}}{9}\left[\frac{1}{2}+\frac{1}{2\left(k \eta_{c}\right)^{2}}+\cos \left(2 k \eta_{c}\right)\left(\frac{1}{2}-\frac{1}{2\left(k \eta_{c}\right)^{2}}\right)-\frac{\sin \left(2 k \eta_{c}\right)}{k \eta_{c}}\right]\right\},
\end{gathered}
$$

siendo $\lambda_{2}$ bien 1 o 0 .

Es importante señalar que el espectro primordial dado por el modelo cosmológico estándar es de la forma $\Delta^{2}(k)=A_{s} k^{n_{s}-1}$. En el modelo donde el colapso sucede durante radiación, la dependencia en $k$ se da a través del coeficiente $C(k)$ que surge como consecuencia de introducir una propuesta de colapso autoinducido. El paso siguiente sería hacer el correspondiente desarrollo utilizando una métrica de cuasi-de Sitter para describir el escenario de inflación, lo que permitiría contar con un espectro de la forma $\Delta^{2}(k) \simeq \mathcal{A} \widetilde{C}(k) k^{n_{s}-1}$, determinando una dependencia en $k$ tanto a través del índice espectral de las perturbaciones escalares (consecuencia de la métrica de fondo) como también del coeficiente de colapso.

A partir de los cálculos realizados para el caso donde el colapso sucede durante inflación, se puede suponer que la dependencia en $k$ introducida por la dinámica del colapso no diferirá sustancialmente del caso analizado en esta tesis, es decir $\widetilde{C}(k) \simeq C(k)$. Trabajar en un espaciotiempo de Sitter exacto simplifica el desarrollo de cálculo y, mediante la introducción de la propuesta de colapso, se ha podido desarrollar un espectro de potencias que no es invariante de escala. La dependencia en $k$ está dada a través de $C(k)$ y esto permite contrastar con las observaciones actuales aquellos esquemas que resultan viables luego del análisis expuesto. 


\subsubsection{Espectros de potencias y anisotropías angulares}

Con el fin de comparar los resultados obtenidos para el modelo de colapso en radiación con las observaciones, se define un modelo fiducial determinado por (4.106). El tiempo de colapso se parametriza del siguiente modo:

$$
\eta_{\vec{k}}^{c}=A
$$

siendo A una constante, ya que como se ha visto en la sección anterior el tiempo de colapso debe ser independiente del modo $k$.

La figura 4.16 muestra (izquierda) el espectro primordial de potencias para el caso en el que el colapso de la función de onda del campo inflatón suceda durante el período de radiación, considerando distintos valores para $A$ y $\lambda_{2}=0$. De la ecuación (4.174) se puede apreciar que la contribución principal a $C(k)$ proviene del término $\left(1+2 \beta_{k}\right) \simeq 1+10^{5} z_{k}^{2}$, por lo que si $\lambda_{2} \neq 0$ el espectro primordial de potencias no tiene cambios significativos. A la derecha, se muestra el espectro primordial de potencias de los modelos de colapso $\left(n_{s}=1\right)$ para distintos valores de $A$ comparado con el del modelo fiducial $\left(n_{s}=0.96\right)$. Observando las escalas en el eje de las ordenadas para ambas figuras puede apreciarse que variar el tiempo de colapso no produce apartamientos significativos respecto a un espectro invariante de escala. Esto sucede porque la restricción estipulada sobre el tiempo de colapso $\eta_{\vec{k}}^{c} \ll 10^{-2} \mathrm{Mpc}$ limita los posibles valores para $A$ e impide con ello grandes apartamientos del modelo fiducial.
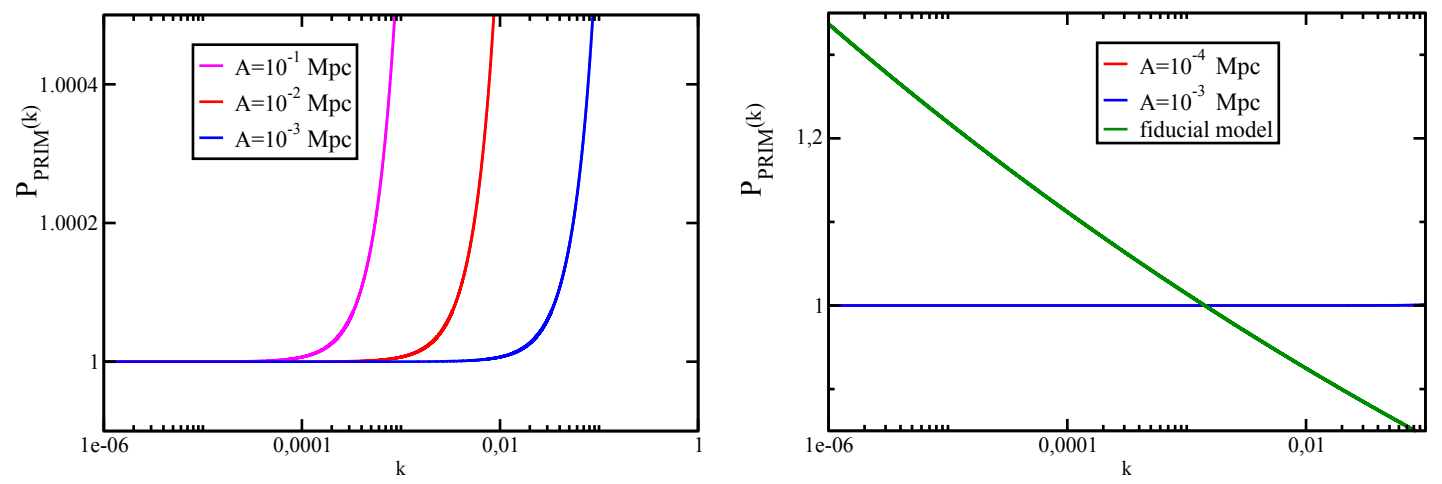

Figura 4.16. A la izquierda: Espectro primordial de potencias para el caso donde el colapso sucede durante la era de radiación, para diferentes valores del tiempo de colapso $\eta_{\vec{k}}^{c}=A$ y $\lambda_{2}=0$. Derecha: Espectro primordial de potencias para el modelo cosmológico estándar $\left(n_{s}=0.96\right)$ en comparación con dos espectros de potencias correspondientes al colapso para el caso de Sitter exacto $\left(n_{s}=1\right)$. León et al. (2014).

En la figura 4.17 se representan las anisotropías angulares en temperatura para los modelos propuestos junto con el modelo fiducial a modo de comparación. La autocorrelación para los modos E y la correlación cruzada entre temperatura y modos E se muestra en la figura 4.18. Puede verse que no hay diferencia entre los modelos propuestos para el colapso durante la época dominada por la radiación y sí un apartamiento respecto del modelo fiducial, tal como se discutió para el espectro primordial.

Si bien la diferencia entre los modelos de colapso y el modelo fiducial de las figuras $4.17 \mathrm{y}$ 4.18 es pequeña, los datos del FCR cuentan actualmente con una gran precisión y restringen fuertemente la viabilidad de las predicciones teóricas de los modelos cosmológicos.

En resumen, la propuesta donde el colapso de la función de onda del campo inflatón tiene lugar en la época de radiación desarrollada para el caso donde la métrica de fondo para 


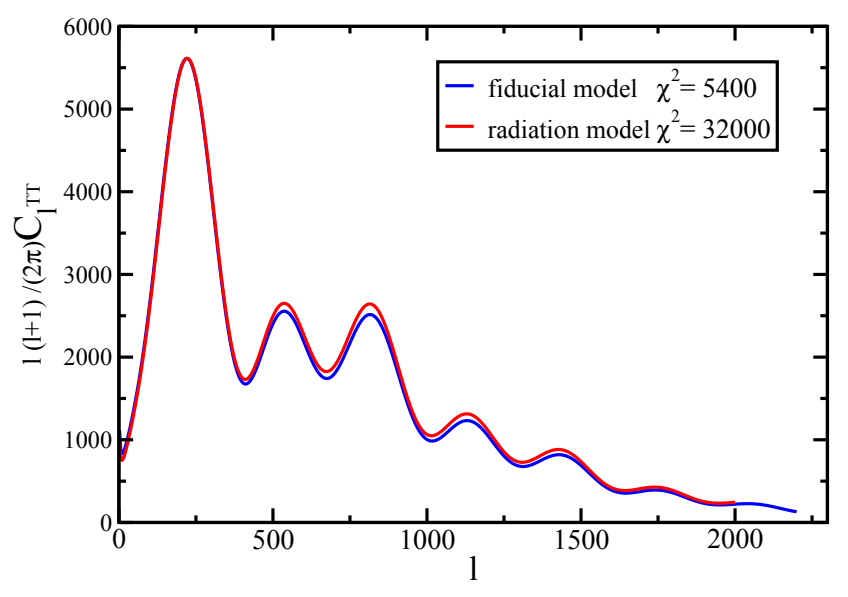

Figura 4.17. Comparación para el espectro de potencias para la autocorrelación angular en la temperatura entre los modelos de colapso de Sitter exacto en radiación $\left(\eta_{\vec{k}}^{c}=10^{-3} \mathrm{Mpc}\right)$ y el modelo fiducial. La normalización es tal que el máximo del primer pico coincida con el del modelo de referencia. Se calcula el coeficiente $\chi^{2}$ que resulta de la comparación de los modelos con datos de Planck2013. León et al. (2014)
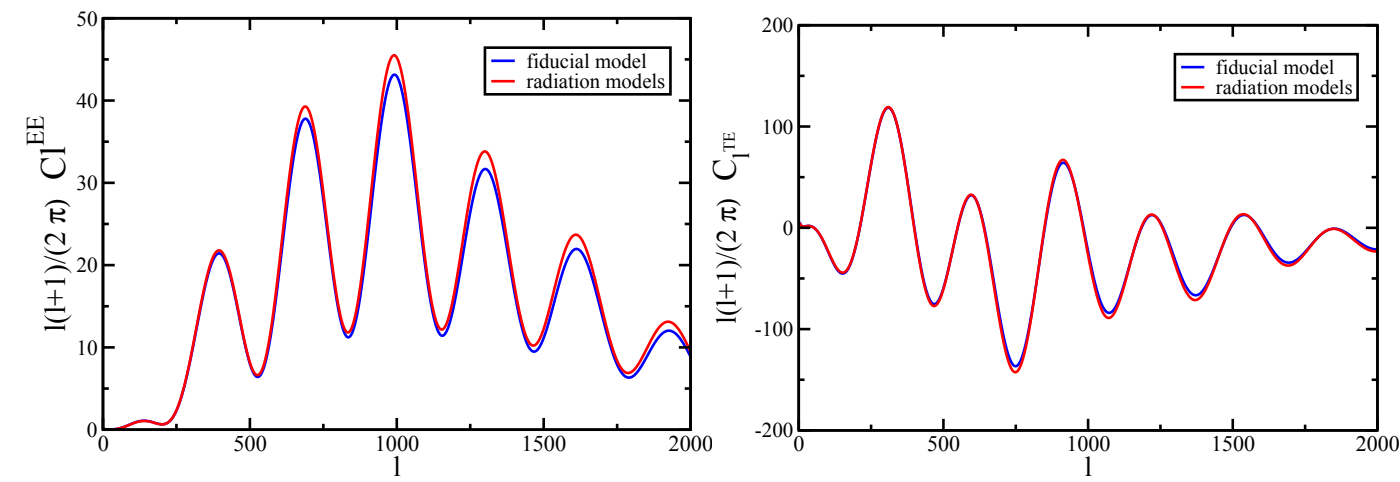

Figura 4.18. Izquierda: espectro angular de potencias para la autocorrelación en los modos E. Derecha: espectro angular de potencias para la correlación cruzada entre la temperatura y los modos E. Se muestran el modelo fiducial y un modelo donde el colapso de la función de onda del campo inflatón tiene lugar durante la era de radiación a un tiempo conforme $\eta_{\vec{k}}^{c}=10^{-3} \mathrm{Mpc}$. La normalización y los valores para $\chi^{2}$ son los mismos que en la figura 4.17. León et al. (2014)

el período inflacionario es un espacio-tiempo de Sitter exacto da como resultado espectros primordiales de potencias invariantes de escala. El mismo se corresponde exactamente con el espectro de un modelo cosmológico estándar desarrollado bajo el mismo formalismo, lo cual no resulta de interés contrastar con los datos ya que un espectro totalmente invariante de escala está excluido con mucha certeza por las observaciones actuales. En base a los resultados obtenidos para el caso donde el colapso se produce en inflación, se espera que la predicción para $P(k)$ en el caso cuasi-de Sitter no difiera significativamente de la correspondiente al modelo fiducial. La diferencia entre ambos modelos radica entonces en que el modelo de colapso permite dar una explicación detallada de la emergencia de un Universo con estructuras a partir de un estado inicial isótropo y homogéneo. 


\subsection{Selección de Modelos Teóricos para el análisis estadístico}

Hasta aquí se han descripto las bases teóricas de cada modelo propuesto, junto con un análisis de su viabilidad. El siguiente paso es comparar el modelo con los datos observacionales y restringir sus parámetros libres.

La tabla 4.2 lista todos los modelos estudiados y si se procederá o no su análisis en la siguiente etapa. En los casos en que no se prosigue su estudio, las razones se fueron dado oportunamente y se resumen a continuación:

\begin{tabular}{lcc}
\hline Modelos/Esquema & cond. horizonte & análisis estad. \\
\hline Independiente & fuera & NO \\
Independiente & dentro & NO \\
Newton & fuera & NO \\
Newton & dentro & SI \\
Wigner & fuera & NO \\
Wigner & dentro & SI \\
CSL & - & SI \\
Radiación & todos & NO \\
\hline
\end{tabular}

Tabla 4.2. Lista de los modelos teóricos de colapso analizados en el presente capítulo y la selección de cuáles serán sometidos al correspondiente análisis estadístico para la estimación de sus parámetros libres. Los motivos por los que se elije no continuar el análisis son diferentes y se distinguen con colores en esta tabla.

Los modelos son indistinguibles del modelo cosmológico estándar: En el estudio de los modelos teóricos se han analizado esquemas tales que sus predicciones no pueden distinguirse de las del modelo cosmológico estándar para un amplio rango de valores del parámetro libre de colapso. Este es el caso de los modelos Independiente tanto en los casos donde el colapso sucede dentro como fuera del horizonte (la longitud de onda del modo propio es más grande o más pequeña que el radio de Hubble respectivamente). Esto implica que es posible estimar el parámetro $B$ de colapso y obtener un modelo teórico cuyas predicciones respecto a las observaciones son exactamente las mismas. La discusión acerca de la preferencia de un modelo frente a otro yace en el terreno teórico: ¿es preferible frente al modelo estándar una propuesta que da una explicación precisa al surgimiento de estructuras a costa de el agregado de nuevos parámetros libres? Esta es una discusión que sigue abierta

Modelo fuera del horizonte: Además del esquema Independiente, se han analizado los esquemas de Newton y Wigner, también en los casos en que el colapso ocurre dentro y fuera del horizonte. Para este último, se necesita estudiar con cuidado la normalización previamente ya que depende del valor de $A$ como parámetro de colapso y puede variar en varios órdenes de magnitud, corriendo el riesgo de introducir errores numéricos en los códigos de programación. Esto es simplemente un detalle técnico que no hace, bajo ningún punto de vista, que un modelo sea preferible sobre otro. 
Por otro lado, en el marco del modelo cosmológico estándar las perturbaciones de la curvatura son evaluadas al momento en que el modo "cruza" el horizonte, ya que una vez fuera éste se congela y no contribuye en los observables. Por ende, los procesos físicos de relevancia ocurren cuando las longitudes propias de los modos son menores al horizonte de Hubble. A efectos de establecer un paralelo con el modelo cosmológico estándar, se restringirá el análisis estadístico a los esquemas "dentro" del horizonte, sin que esto implique que los modelos de Newton y Wigner "fuera" del horizonte deban ser descartados.

Colapso durante la época dominada por radiación: La propuesta de que el colapso de la función de onda del campo inflatón tenga lugar durante el período en que el Universo está dominado por la radiación implica muchas restricciones. Esto hace que el parámetro libre de este modelo no pueda tomar valores tales que la forma funcional del espectro primordial de potencias pueda apartarse del modelo fiducial y obtener así alguna predicción que lo distinga del mismo. Entonces, en los casos en los que el colapso en radiación resulta viable (tabla 4.1) el modelo es prácticamente igual observacionalmente al modelo cosmológico estándar y la discusión es similar a lo que ocurre con el esquema Independiente.

En el capítulo que sigue se realiza el análisis estadístico de estimación de parámetros para los siguientes modelos:

- Independiente dentro del horizonte (al cual se hará referencia como Independiente)

- Wigner dentro del horizonte (simplemente Wigner de ahora en más)

- CSL

Cada uno de estos modelos será contrastado con distintos conjuntos de datos observacionales recientes y de interés cosmológico según se detalló en el capítulo 1. 



\section{Capítulo 5}

\section{Resultados}

En este capítulo se exponen los resultados obtenidos en los análisis estadísticos para los distintos modelos propuestos de colapso. Cada sección se encabezará con un recordatorio de los detalles principales de cada modelo y los datos utilizados. Se destinan apartados especiales para la comparación de distintos modelos con un mismo conjunto de datos y para un mismo modelo con distintos conjuntos de datos.

De todas las propuestas descriptas a lo largo de esta tesis, sólo llegan a esta instancia de análisis aquellas que resultan viables y además otorgan un grado de predictibilidad al modelo que permite establecer una diferenciación respeto al modelo cosmológico estándar. Esto se debe a la alta demanda de recursos computacionales necesaria para correr las cadenas de Markov-Monte Carlo. Resulta imprescindible una evaluación previa para no invertir tiempos de trabajo innecesarios.

Los resultados se presentan en gráficos triangulares donde se exhiben sobre la diagonal las probabilidades unidimensionales a posteriori de los parámetros cosmológicos de interés. Las figuras fuera de la diagonal corresponden a las proyecciones de las probabilidades conjuntas $a$ posteriori de dos parámetros que se corresponden según las entradas de la tabla. Se muestran los contornos de confianza correspondiente a los límites de probabilidad de $68 \%$ y $95 \%$.

La ejecución del CosmoMC se realizó en el cluster Miztli de la UNAM, cuyo tiempo de cómputo se obtuvo mediante solicitudes de recursos renovadas anualmente y el acceso al mismo fue posible gracias a la colaboración establecida con investigadores de esa institución. Los gráficos se construyeron en computadoras personales con herramientas de python provistas en el mismo paquete del CosmoMC, mientras que los análisis de convergencia pudieron llevarse acabo en ambas plataformas.

El Nuevo Equipo de Supercómputo (NES) de la UNAM denominado Miztli (figura 5.1) es un sistema HP Cluster Platform 3000SL con una capacidad de procesamiento de 118 TFlop/s (118 billones de operaciones aritméticas por segundo). Cuenta con 5312 núcleos de procesamiento Intel E5-2670, 16 tarjetas NVIDIA m2090, una memoria RAM total de 15000 Gbytes y un sistema de almacenamiento masivo de 750 Terabytes. Se compone de 344 servidores (HP Proliant SL230 y SL250), comunicados a través de una red de datos Infiniband, una red de administración ethernet, una red de consolas y varios sistemas de archivos globales.

Este poder de cómputo permitió obtener resultados en plazos de entre 3 y 10 días, a comparación de otros clusters donde llegaron a demorar más de un mes. Se precisaron entre 6 y 16 GB de memoria RAM para almacenar los datos que son comparados a cada instante 


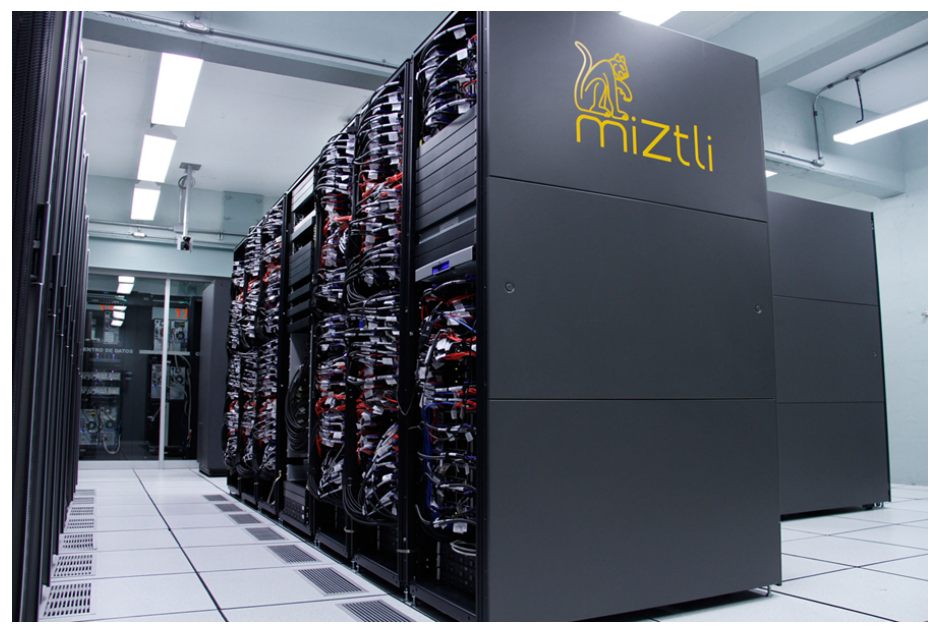

Figura 5.1. Supercomputadora Miztli - UNAM. Fuente: www.super.unam.mx.

con las predicciones teóricas. Entre resultados y pruebas realizadas se necesitaron unos pocos cientos GygaBites de almacenamiento para dar lugar al código, datos y salidas del programa.

Los cómputos de la evidencia bayesiana para la comparación entre modelos fueron obtenidos por Micol Benetti, colaboradora del grupo en el Observatorio Nacional de Río de Janeiro. La tabla de referencia respecto a los resultados de este indicador es la 2.1 y se encuentra en el capítulo 2 .

En los análisis estadísticos realizados se consideraron los siguientes parámetros libres del modelo cosmológico estándar:

$\Omega_{b} h^{2}$ : densidad de materia bariónica en unidades de $h^{2}$,

$\Omega_{c} h^{2}$ : densidad de materia oscura en unidades de $h^{2}$,

$\tau$ : profundidad óptica de reionización,

$\theta$ : tamaño angular del horizonte de sonido al momento del desacople,

$\ln \left(10^{10} A_{s}\right)$ : escala de las perturbaciones escalares,

$n_{s}$ : índice espectral de las perturbaciones escalares,

recordando que $h$ está definida como:

$$
h=\frac{H_{0}}{100 \mathrm{~km} \mathrm{~s}^{-1} \mathrm{Mpc}^{-1}},
$$

siendo $H_{0}$ el valor actual de la constante de Hubble. Los parámetros libres de los modelos de colapso son:

$B$ : apartamiento correspondiente a los modelos cuasi-de Sitter,

$\alpha$ : coeficiente para los modelos CSL.

Además se acompaña en los casos donde resulte de interés, la diferencia entre los resultados del test de $\chi^{2}$ construidas del siguiente modo:

$$
\Delta \chi^{2}=\chi_{\text {canónico }}^{2}-\chi_{\text {colapso }}^{2},
$$

donde se define $\chi^{2}=-2 \ln (\mathcal{L})$, siendo $\mathcal{L}$ la función likelihood. 


\subsection{Esquemas de colapso}

La extensión al caso de cuasi-de Sitter en el caso que el colapso se produzca durante inflación es uno de los avances que comprenden este trabajo. Según el análisis llevado a cabo en el capítulo 4, el tiempo propio de colapso se parametriza:

$$
\eta=\frac{A}{k}+B
$$

Si bien los parámetros libres a determinar son dos, sólo se somete a análisis $B$ que es un pequeño apartamiento del modelo estándar. La variable $A$ funciona como una normalización que es absorbida por $A_{s}$, razón por la cual ambas están fuertemente correlacionadas. Es importante recordar que fijando $B=0$ se recupera la forma funcional para el espectro primordial de potencias del modelo cosmológico estándar.

Los esquemas que llegaron hasta esta instancia de análisis son dos: Newton y Wigner, cada uno con una propuesta diferente para describir el colapso de la función de onda del campo inflatón dentro del período inflacionario. Sólo se consideran aquellos casos donde el colapso se produce antes de que el modo cruce el horizonte. Para diferenciar los conjuntos de datos utilizados en cada análisis estadístico, se expondrán los gráficos en distintas secciones.

\subsubsection{Análisis estadísticos con datos de Planck2015}

En cada caso se establece como modelo de control y comparación un análisis estadístico realizado con el mismo conjunto de datos sobre un modelo cosmológico estándar, al que se denomina modelo fiducial.

Los datos de Planck2015 incluyen mediciones para las anisotropías en temperatura, los modos E de polarización y la correlación cruzada entre ambos (ver sección 1.7). Se utiliza el conjunto de datos para la temperatura entre los multipolos $30<l<2500$, y estimaciones de temperatura y polarización para $1<l<30$ (ver sección 1.6). La tabla 5.1 indica la estimación de los parámetros cosmológicos para el modelo fiducial definido por este conjunto de datos. Es esta tabla, así como también en las secciones subsiguientes, se incluirán también los resultados para $H_{0}$ que es un parámetro derivado de $\theta$.

\begin{tabular}{cr}
\hline Parámetro & Estimación \\
\hline$n_{s}$ & $0.9656_{-0.0063}^{+0.0064}$ \\
$\ln \left(10^{10} A_{s}\right)$ & $3.0906_{-0.0362}^{+0.0368}$ \\
$\Omega_{b} h^{2}$ & $0.0222_{-0.0002}^{+0.0002}$ \\
$\Omega_{c} h^{2}$ & $0.1197_{-0.0022}^{+0.0022}$ \\
$100 \theta_{M C}$ & $1.0409_{-0.0005}^{+0.0005}$ \\
$\tau$ & $0.0782_{-0.0205}^{+0.0187}$ \\
$H_{0}$ & $67.3257_{-0.9916}^{+1.0030}$ \\
\hline
\end{tabular}

Tabla 5.1. Estimación de los datos cosmológicos en el marco del modelo cosmológico estándar con los datos de Planck2015. 


\section{NEWTON}

En el esquema de Newton la variable que colapsa es el momento conjugado del campo inflatón (ver sección 4.4.2). La figura 5.2 muestra la proyección de las probabilidades a posteriori de los parámetros cosmológicos, restringiendo sus valores en cada caso. El valor que corresponde al parámetro de colapso $A$ se fija en 650 , como resultado de un análisis previo donde se evalúan distintas combinaciones entre $A$ y $A_{s}$. El valor seleccionado para $A$ es aquel que (fijando $B=0$ ) predice un espectro primordial de potencias cuya normalización implica un valor de $A_{s}$ muy similar al del modelo fiducial.

Los resultados obtenidos para los parámetros $\Omega_{b} h^{2}, \Omega_{c} h^{2}, \tau, H_{0}$ y $n_{s}$ son consistentes a $2 \sigma$ con aquellos obtenidos para el modelo fiducial. De la observación de los gráficos unidimensionales puede verse que en estos casos el valor más probable coincide pero no así la dispersión, permitiendo ampliar el intervalo de confianza de los parámetros cosmológicos.

Se observa que las modificaciones introducidas por el mecanismo de colapso permiten un mayor intervalo de confianza del índice espectral de las perturbaciones escalares $n_{s}$, el cual depende directamente del modelo particular de inflación. Esto sugiere que potenciales $V(\phi)$ de inflación que fueron descartados en el contexto del modelo estándar no lo sean en el marco de los modelos de colapso.

En la figura 5.3 se observan en detalle los contornos de confianza de los parámetros cosmológicos cuya estimación resulta de particular interés en el esquema de Newton. Se destaca una predicción distinta para el valor de $A_{s}$, lo cual es consecuencia de la degeneración que existe entre este parámetro y $A$, según se discutió en el capítulo 4 . El parámetro $A_{s}$ determina de manera indirecta la escala de $V(\phi)$ dado que $A_{s} \simeq V(\phi) / \varepsilon_{1}$. El valor de $\varepsilon_{1}$ puede ser determinado si se detectan los modos $\mathrm{B}$ de polarización. Por lo tanto, hasta que no se realice una detección de los mismos, el valor de $A_{s}$ no puede determinar la escala del potencial de inflación y, en consecuencia, la diferencia en la determinación del valor de $A_{s}$ (modelos de colapso vs. modelo fiducial) no tiene una interpretación en términos de la física del período inflacionario.

Por último, se observan las estimaciones para los parámetros cosmológicos al $68 \%$ de probabilidad en la tabla 5.2. Si bien $B=0$ es un valor posible, no es el más probable.

Respecto a la estimación del parámetro $B$ de colapso, es importante destacar que la herramienta de análisis estadístico utilizada asume que la probabilidad a posteriori es gaussiana. La probabilidad a posteriori correspondiente a $B$ (figura 5.3) no es exactamente gaussiana. Algo similar ocurre para los resultados del esquema de Wigner. Para una mejor determinación de los límites sobre $B$, se han realizado integraciones y estimaciones manuales para algunos casos particulares obteniendo exactamente los mismos intervalos de confianza para el $68 \%$ de probabilidad. Por ende, resultan confiables los resultados obtenidos del procesamiento habitual de estimación estadística provista por el código getdist para los parámetros de colapso que se muestran en las tablas.

De los resultados obtenidos por el CosmoMC se calcula la diferencia entre los $\chi^{2}$ del modelo cosmológico estándar y el modelo de colapso bajo estudio (ecuación (5.2)) Complementando esta información, se calcula también la evidencia Bayessiana utilizando el modelo cosmológico estándar como modelo de control y comparación. Para el caso del esquema de Newton estos indicadores toman los siguientes valores:

$$
\begin{aligned}
\Delta \chi^{2} & =1.03 \\
\ln \mathcal{B}_{i j} & =-3.33
\end{aligned}
$$




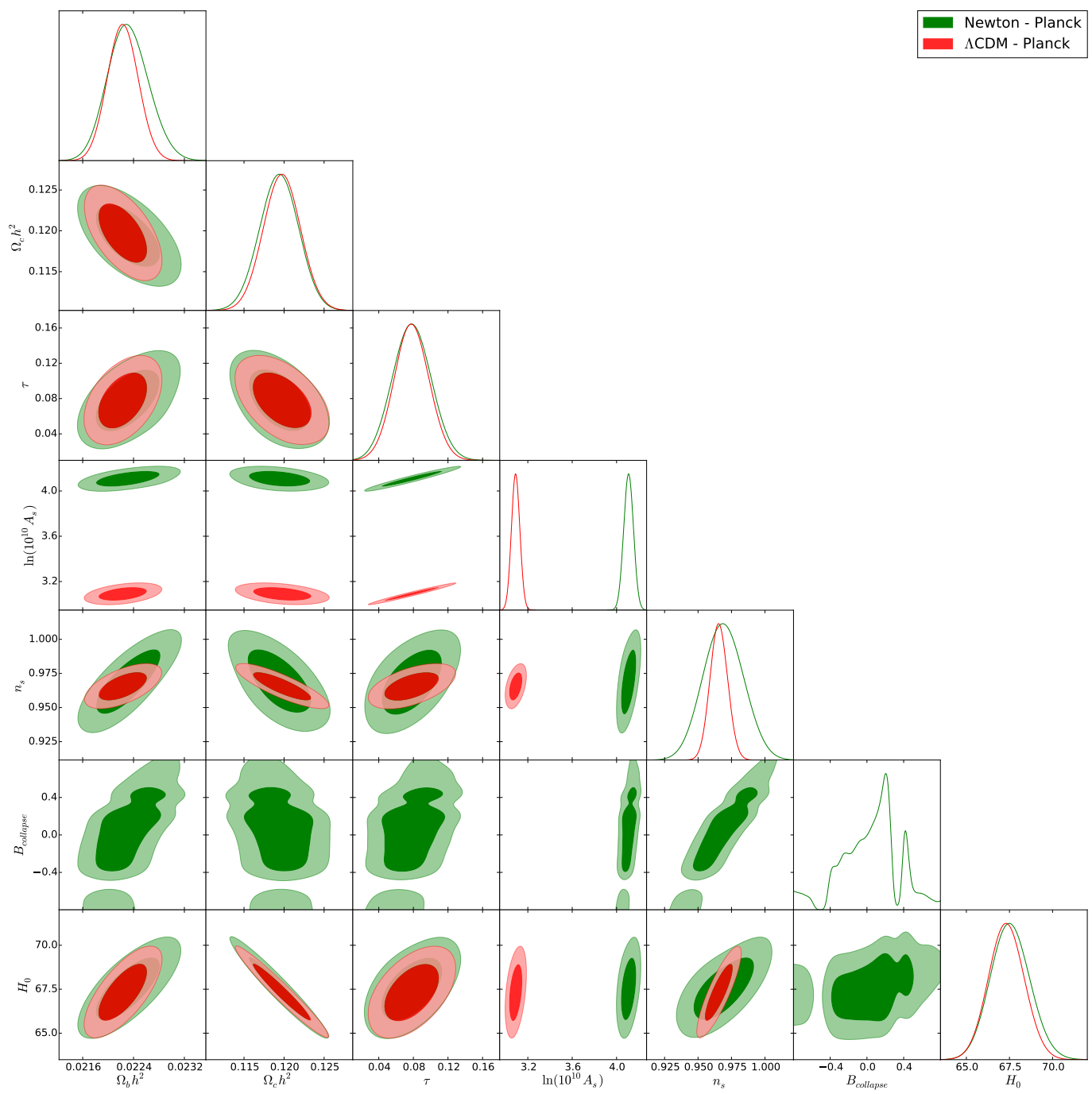

Figura 5.2. Distribuciones a posteriori marginalizadas para el modelo de Newton obtenidas con los datos de Planck2015. En la diagonal se muestran las distribuciones de densidad de probabilidad marginalizadas para un parámetro mientras que los otros paneles muestran los contornos de confianza a $1 \sigma$ y $2 \sigma$ de pares de los parámetros cosmológicos.

El factor de Bayes indica una preferencia moderada por el modelo cosmológico estándar.

\section{WIGNER}

En el esquema de Wigner (recordando la sección 4.4.2) colapsan de modo dinámico tanto la variable del campo inflatón como su momento conjugado, relacionadas a través de una distribución de Wigner. De este modo, ambas variables resultan correlacionadas.

Los resultados obtenidos en las estimaciones de los parámetros cosmológicos son consistentes con los del modelo cosmológico estándar (figura 5.4).

Se observa nuevamente una ampliación en el intervalo de confianza para los valores de $n_{s}$ (figura 5.5). A diferencia del esquema anterior, los valores para $\ln \left(10^{10} A_{s}\right)$ se superponen aunque no coindicen exactamente. Esto se debe a que la modificación introducida en este esquema permite acercarse a valores numéricos de $A_{s}$ similares a los reportados por el modelo 


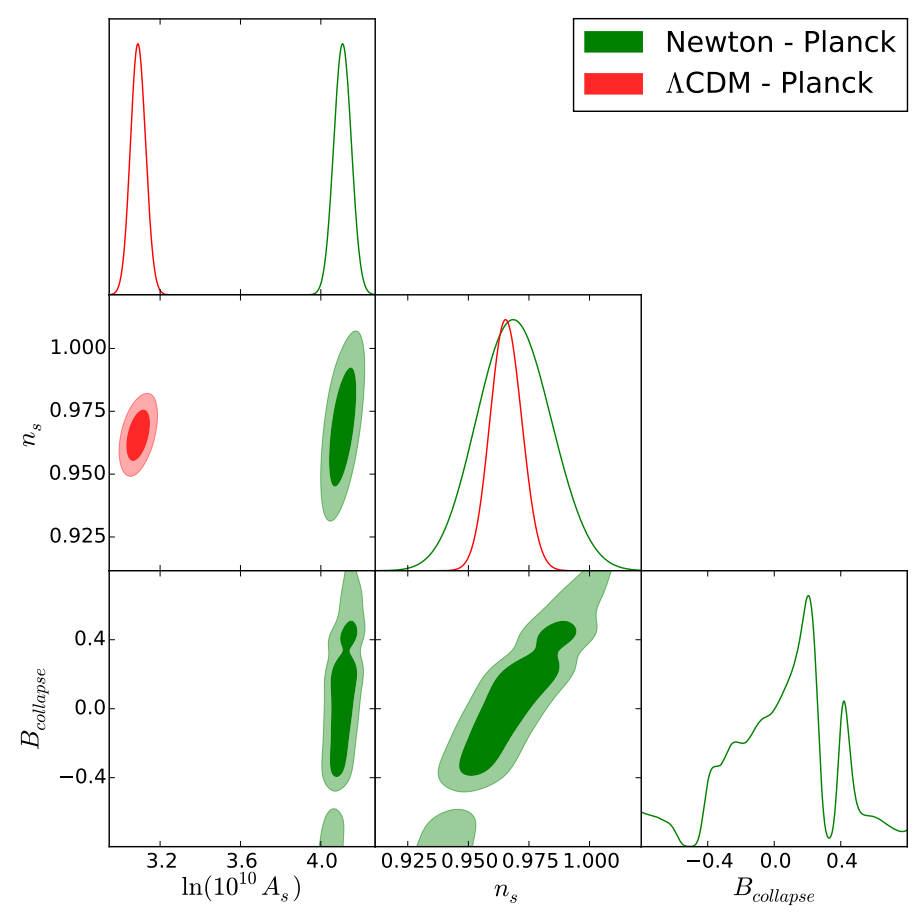

Figura 5.3. Distribuciones a posteriori marginalizadas para el modelo de Newton obtenidas con los datos de Planck2015. En la diagonal se muestran las distribuciones de densidad de probabilidad marginalizadas para un parámetro mientras que los otros paneles muestran los contornos de confianza a $1 \sigma$ y $2 \sigma$ de pares de los parámetros $\ln \left(10^{10} A_{s}\right), n_{s}$ y $B$.

\begin{tabular}{cr}
\hline Parámetro & Valor Medio $\pm 1 \sigma$ \\
\hline$B_{\text {collapse }}$ & $0.0499_{-0.3113}^{+0.3500}$ \\
$n_{s}$ & $0.9689_{-0.0147}^{+0.0147}$ \\
$\ln \left(10^{10} A_{s}\right)$ & $4.1082_{-0.0421}^{+0.0419}$ \\
$\Omega_{b} h^{2}$ & $0.0223_{-0.0003}^{+0.0003}$ \\
$\Omega_{c} h^{2}$ & $0.1194_{-0.0023}^{+0.0023}$ \\
$100 \theta_{M C}$ & $1.0409_{-0.0005}^{+0.0005}$ \\
$\tau$ & $0.0784+0.0214$ \\
$H_{0}$ & $67.5440_{-1.1898}^{+1.0972}$ \\
\hline
\end{tabular}

Tabla 5.2. Estimaciones de los parámetros cosmológicos para el esquema de Newton bajo la aproximación semiclásica en el caso de que el colapso tenga lugar "dentro" del horizonte, utilizando los datos Planck2015.

estándar antes de introducir cualquier normalización. A tal efecto se fija $A=-749.935$ y la estimación de los parámetros cosmológicos se lista en la tabla 5.3. Se observa que si bien $B=0$ se encuentra dentro del intervalo de confianza y es, por lo tanto, un valor posible, no es el más probable.

En cuanto a la comparación del $\chi^{2}$ con el modelo estándar y su factor de Bayes, los 


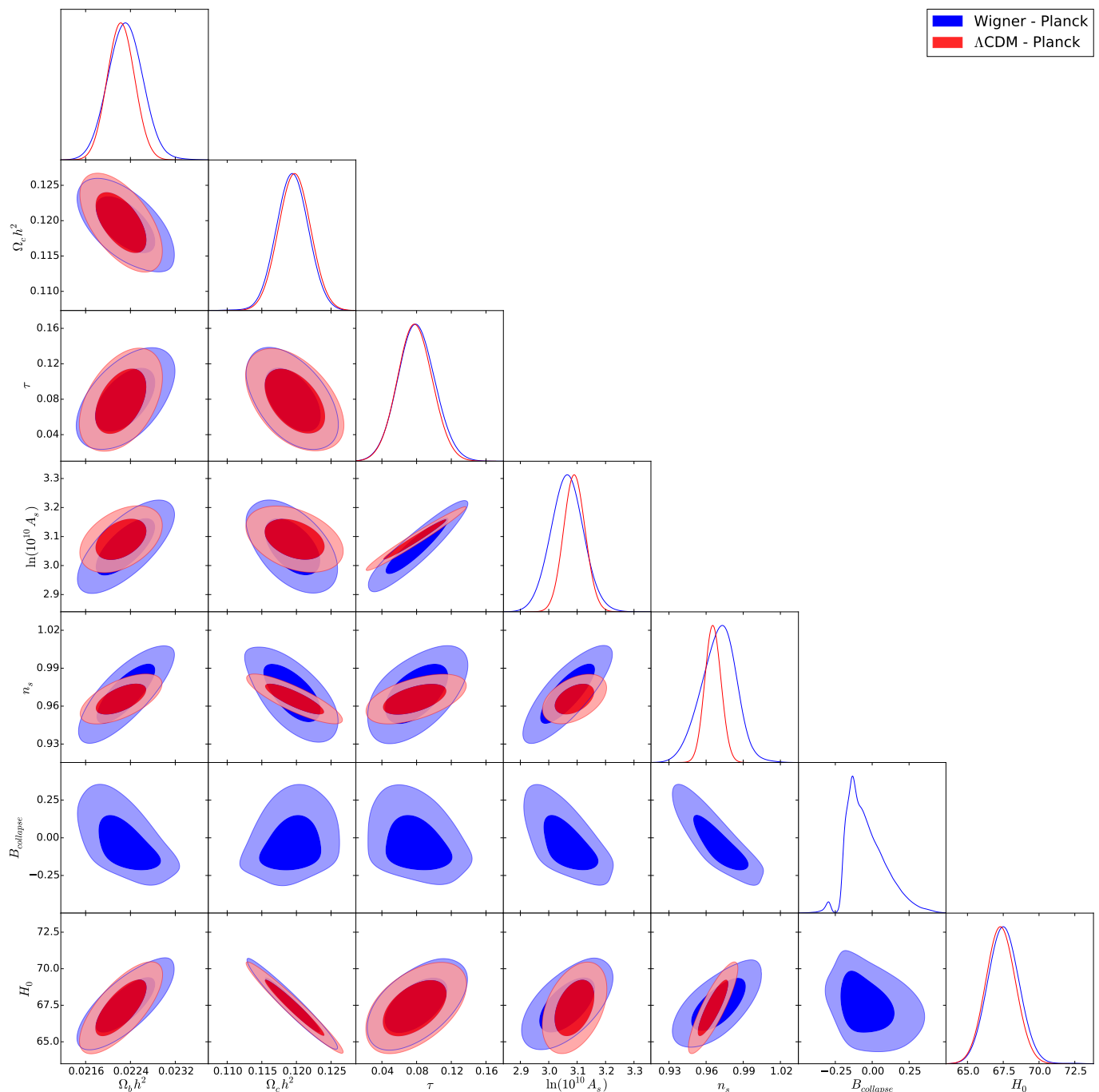

Figura 5.4. Distribuciones a posteriori marginalizadas para el modelo de Wigner obtenidas con los datos de Planck2015. En la diagonal se muestran las distribuciones de densidad de probabilidad marginalizadas para un parámetro mientras que los otros paneles muestran los contornos de confianza a $1 \sigma$ y $2 \sigma$ de pares de los parámetros cosmológicos.

resultados son:

$$
\begin{aligned}
\Delta \chi^{2} & =1.88 \\
\ln \mathcal{B}_{i j} & =-1.44
\end{aligned}
$$

El facto de bayes indica preferencia moderada por el modelo cosmológico estándar.

\section{Comparación entre modelos}

La figura 5.6 muestra los resultados obtenidos para los esquemas de Newton y Wigner en un sólo gráfico. En primer lugar se observa la diferencia entre ambos valores de $\ln \left(10^{10} A_{s}\right)$ discutida anteriormente y similares predicciones para los intervalos de confianza de $n_{s}$, aunque con distinta correlación respecto a $B$. Los parámetros $n_{s}$ y $B$ se encuentran degenerados, pero con pendientes opuestas según el modelo. 


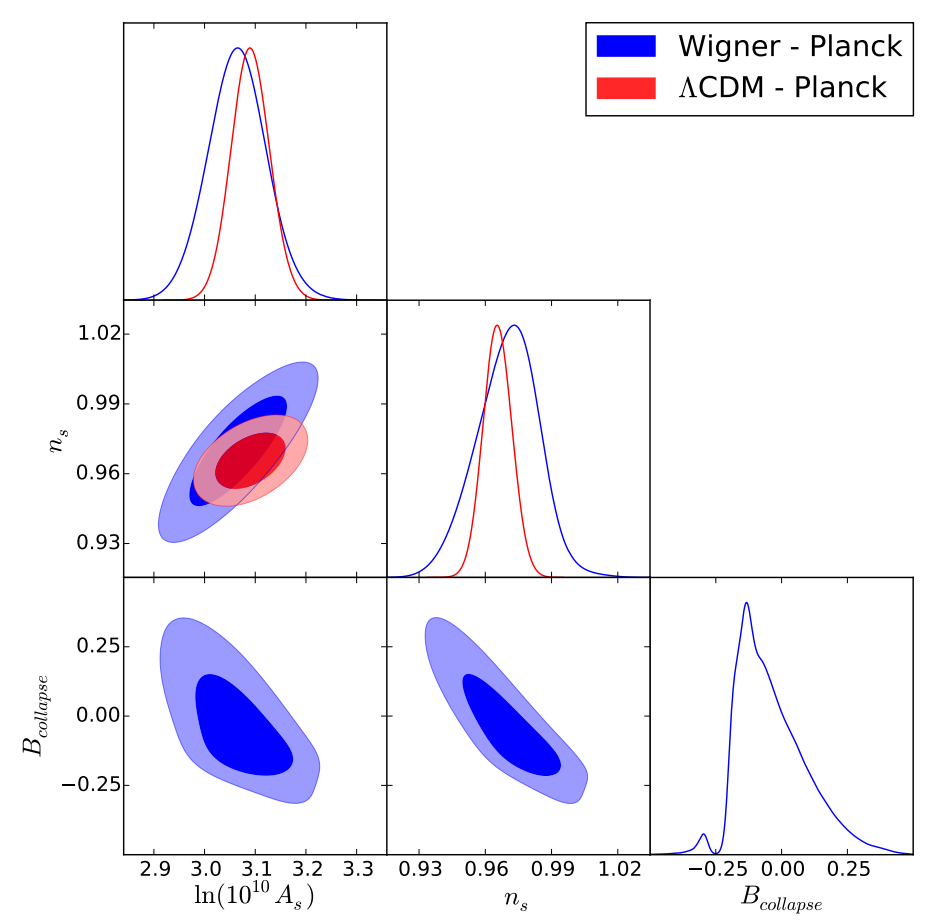

Figura 5.5. Distribuciones a posteriori marginalizadas para el modelo de Wigner obtenidas con los datos de Planck2015. En la diagonal se muestran las distribuciones de densidad de probabilidad marginalizadas para un parámetro mientras que los otros paneles muestran los contornos de confianza a $1 \sigma$ y $2 \sigma$ de pares de los parámetros $\ln \left(10^{10} A_{s}\right), n_{s}$ y $B$.

\begin{tabular}{cr}
\hline Parámetro & Valor Medio $\pm 1 \sigma$ \\
\hline$B_{\text {collapse }}$ & $-0.0369_{-0.1604}^{+0.0841}$ \\
$n_{s}$ & $0.9700_{-0.0124}^{+0.0157}$ \\
$\ln \left(10^{10} A_{s}\right)$ & $3.0648_{-0.0563}^{+0.0552}$ \\
$\Omega_{b} h^{2}$ & $0.0223_{-0.0003}^{+0.0003}$ \\
$\Omega_{c} h^{2}$ & $0.1194_{-0.0023}^{+0.0023}$ \\
$100 \theta_{M C}$ & $1.0409_{-0.0005}^{+0.0005}$ \\
$\tau$ & $0.080{ }_{-0.0206}^{+0.0208}$ \\
$H_{0}$ & $67.5270_{-1.0741}^{+1.0688}$
\end{tabular}

Tabla 5.3. Estimación de parámetros cosmológicos para el esquema de colapso de Wigner según los datos de Planck2015.

\subsubsection{Análisis estadísticos con datos de Planck2015 + BAO}

Como se discutió en la sección 1.8, los datos de BAO (Barionic Acoustic Oscillations) pueden ser utilizados como complemento para el análisis estadístico y permiten restringir aún más el espacio de parámetros. En lo que sigue se describen los resultados del análisis estadístico de los esquemas de Newton y Wigner combinando ambos conjuntos de datos. 


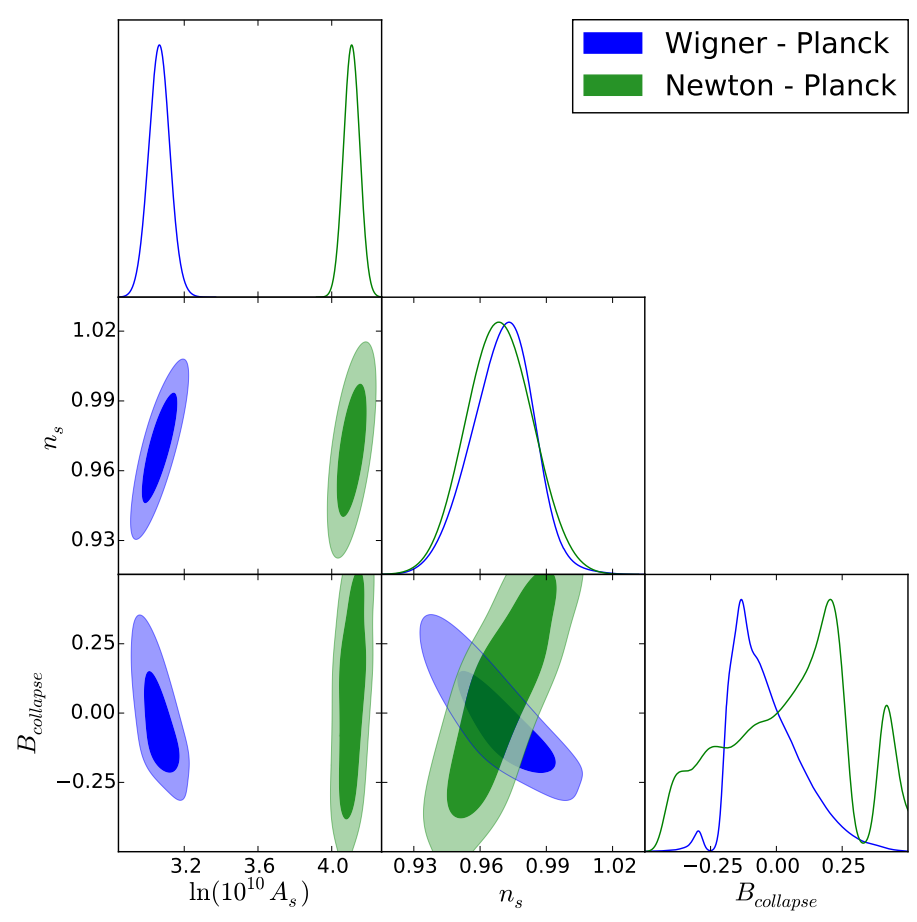

Figura 5.6. Distribuciones a posteriori marginalizadas para los modelos de Newton y Wigner obtenidas con los datos de Planck2015. En la diagonal se muestran las distribuciones de densidad de probabilidad marginalizadas para un parámetro mientras que los otros paneles muestran los contornos de confianza a $1 \sigma$ y $2 \sigma$ de pares de los parámetros $\ln \left(10^{10} A_{s}\right), n_{s} \mathrm{y}$ $B$.

\section{NEWTON}

Se han efectuado los análisis estadísticos correspondientes a los datos conjuntos del FCR y BAO, obteniendo resultados muy similares a la sección 5.1.1. A fin de evitar redundancias, sólo se muestra la comparación de los resultados de aquellos parámetros que más han sido afectados por el colapso para describir cualitativamente los cambios introducidos al incorporar un nuevo conjunto de datos.

En la figura 5.7 se muestran los resultados obtenidos con datos de Planck2015 y Planck2015 $+\mathrm{BAO}$, pudiendo observarse un pequeño corrimiento en el máximo de $n_{s}$ (consistente con los errores) y una disminución de la dispersión en general.

\section{WIGNER}

Los gráficos que muestran los resultados de los análisis estadísticos se pueden ver en las figuras 5.8 y 5.9. En síntesis, algunos valores son levemente distintos respecto al caso de Planck2015 aunque están dentro del error estimado (tabla 5.4). Es importante destacar que en algunos casos, como por ejemplo $\Omega_{c} h^{2}$ y $H_{0}$, se reduce a la mitad la estimación del error en el primer caso y hasta en un orden de magnitud en el segundo. Esto implica que el nuevo conjunto de datos introducido ayuda a restringir con mayor precisión la estimación de las cantidades de interés. Este detalle se puede ver sobre los parámetros $A_{s}, n_{s}$ y $B$ en la figura 5.10 . 


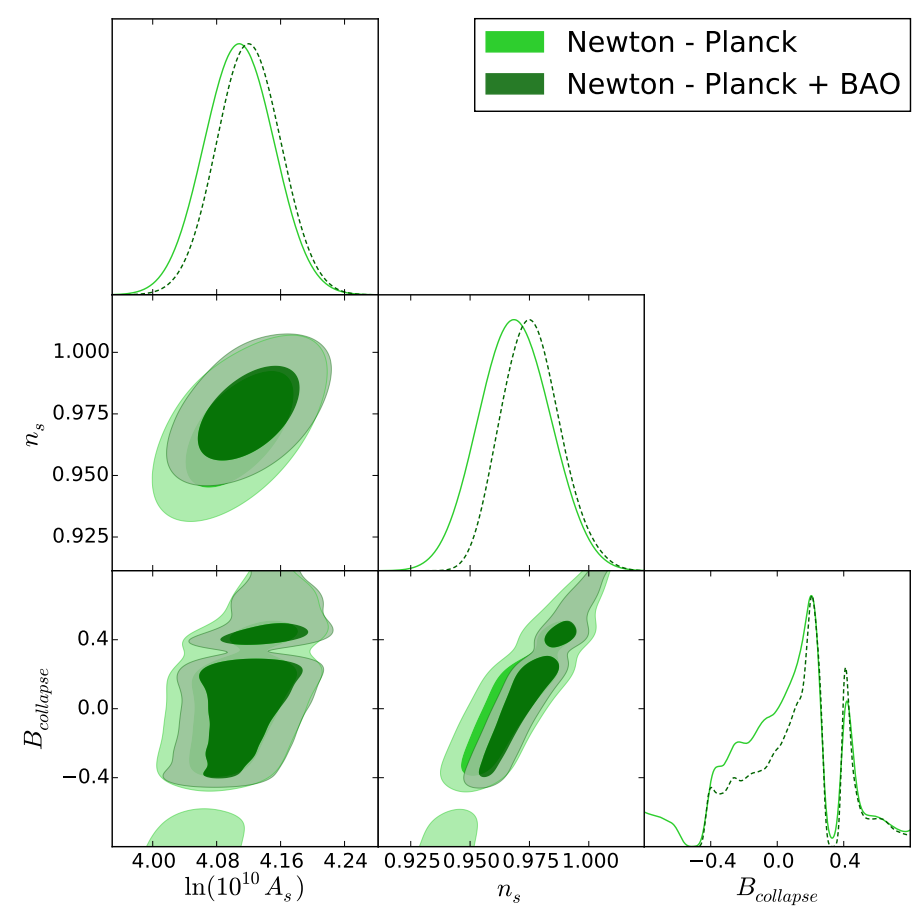

Figura 5.7. Distribuciones a posteriori marginalizadas para el modelo de Newton obtenidas con los datos de Planck2015 y Planck2015+BAO. En la diagonal se muestran las distribuciones de densidad de probabilidad marginalizadas para un parámetro mientras que los otros paneles muestran los contornos de confianza a $1 \sigma$ y $2 \sigma$ de pares de los parámetros $\ln \left(10^{10} A_{s}\right), n_{s}$ y $B$.

\begin{tabular}{cr}
\hline Parámetro & Valor Medio $\pm 1 \sigma$ \\
\hline$B_{\text {collapse }}$ & $-0.0582_{-0.1435}^{+0.0713}$ \\
$n_{s}$ & $0.9752_{-0.0090}^{+0.0132}$ \\
$\ln \left(10^{10} A_{s}\right)$ & $3.0815_{-0.0503}^{+0.0500}$ \\
$\Omega_{b} h^{2}$ & $0.0224_{-0.0003}^{+0.0003}$ \\
$\Omega_{c} h^{2}$ & $0.1180_{-0.0012}^{+0.0012}$ \\
$100 \theta_{M C}$ & $1.0411_{-0.0004}^{+0.0004}$ \\
$\tau$ & $0.0860_{-0.0191}^{+0.0191}$ \\
$H_{0}$ & $68.1865_{-0.5855}^{+0.5910}$ \\
\hline
\end{tabular}

Tabla 5.4. Estimación de los parámetros cosmológicos para el esquema de colapso semiclásico de Wigner con los datos de Planck2015+BAO.

Los indicadores estadísticos estudiados muestran para este modelo:

$$
\begin{aligned}
\Delta \chi^{2} & =0.31 \\
\ln \mathcal{B}_{i j} & =2.96 .
\end{aligned}
$$

Si bien la diferencia entre ambos $\chi^{2}$ es del orden de $6 \times 10^{-3} \%$, esto no puede tomarse como un indicador determinante para preferir un modelo sobre otro, ya que se basan en distinto 


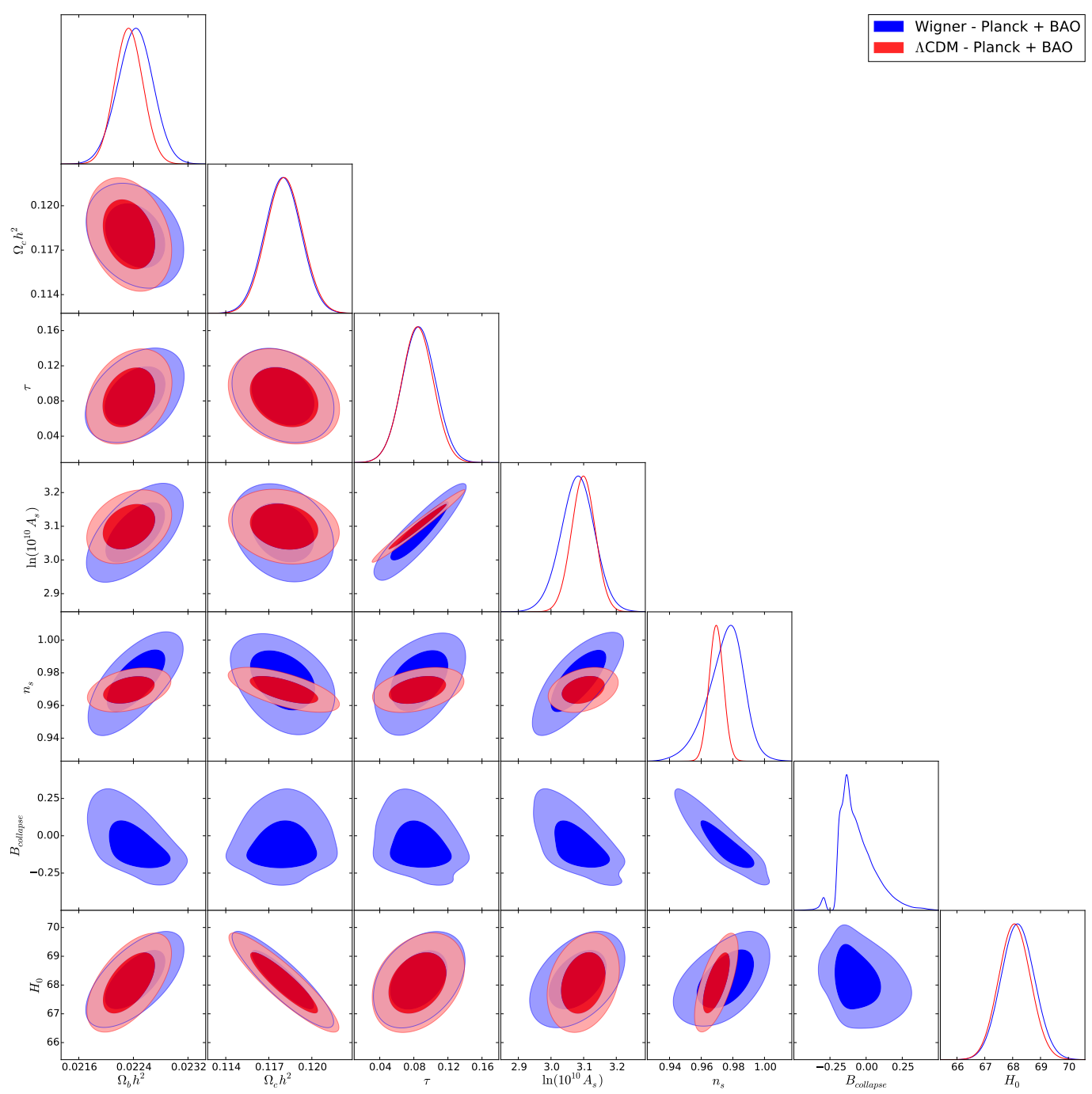

Figura 5.8. Distribuciones a posteriori marginalizadas para el modelo de Wigner obtenidas con los datos de Planck2015+BAO. En la diagonal se muestran las distribuciones de densidad de probabilidad marginalizadas para un parámetro mientras que los otros paneles muestran los contornos de confianza a $1 \sigma$ y $2 \sigma$ de pares de los parámetros cosmológicos.

número de parámetros libres. Por este motivo se calcula la evidencia bayesiana, un indicador que toma en cuenta la diferente cantidad de parámetros libres y puede utilizarse para indicar una ventaja de un modelo frente a otro de referencia (ver discusión en la sección 2.4.1). Según la tabla 2.1, el factor de bayes indica en este caso una preferencia moderada para el esquema de Wigner por sobre el modelo canónico según de los datos conjuntos Planck2015 + BAO.

En este caso no sólo es un modelo viable, si no que también es favorecido moderadamente por los datos a pesar de la incorporación de parámetros extra. Puede afirmarse que cumple el objetivo de representar bien las observaciones y dar un marco teórico más preciso para el surgimiento de las semillas de la estructura a gran escala. 


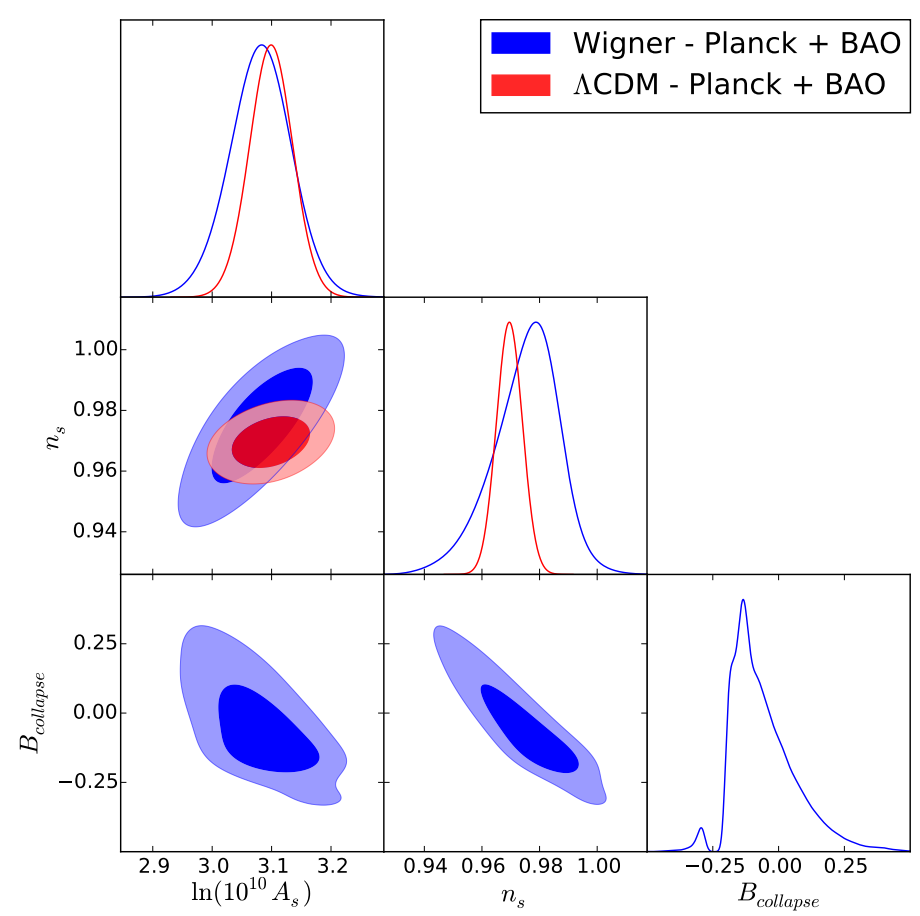

Figura 5.9. Distribuciones a posteriori marginalizadas para el modelo de Wigner obtenidas con los datos de Planck2015+BAO. En la diagonal se muestran las distribuciones de densidad de probabilidad marginalizadas para un parámetro mientras que los otros paneles muestran los contornos de confianza a $1 \sigma$ y $2 \sigma$ de pares de los parámetros $\ln \left(10^{10} A_{s}\right), n_{s} \mathrm{y}$ $B$.

\subsubsection{Análisis estadísticos con datos de Planck2015+BAO +HST}

Dado que los espectros de potencias predichos para los modelos de colapso son diferentes al del modelo cosmológico estándar, puede suceder que la estimación para el parámetro $H_{0}$ también sea distinta. Por ello se incorporan al análisis estadístico los datos del HST (Hubble Space Telescope) con el fin de evaluar si es posible aliviar la tensión entre datos que se discutió en el capítulo 1.

Debido a que la evidencia bayesiana obtenida para el esquema de Wigner con los datos de Planck2015+BAO favorece levemente a este modelo, se elige este esquema para profundizar el análisis e incorporar un tercer conjunto de datos. Se incluirá en esta sección el análisis de este esquema teniendo en cuenta además los datos del Hubble Space Telescope para la determinación de $H_{0}$ (Riess et al. 2016). Según la discusión de la sección 1.9 se busca analizar si es posible resolver la tensión que existe entre las determinaciones de la constante de Hubble con datos del FCR $\left(H_{0}=67.31 \pm 0.96 \mathrm{~km} \mathrm{~s}^{-1} \mathrm{Mpc}^{-1}\right.$,Planck Collaboration et al. (2016b)) y datos pertenecientes al Universo local $\left(H_{0}=73.24 \pm 1.74 \mathrm{~km} \mathrm{~s}^{-1} \mathrm{Mpc}^{-1}\right.$, Riess et al. (2016)).

Los resultados del análisis estadístico se muestran en la figura 5.11. Se exhiben solamente aquellos parámetros que dan cuenta de alguna variación, no se muestran $\Omega_{c} h^{2}$ y $\tau$ por ser esencialmente los mismos que el modelo fiducial. Puede apreciarse que la diferencia en el resto de los parámetros es mínima y no resulta apreciable para la constante de Hubble, de modo que no se encuentran modificaciones tales que puedan aportar a resolver la discrepancia antes mencionada. 


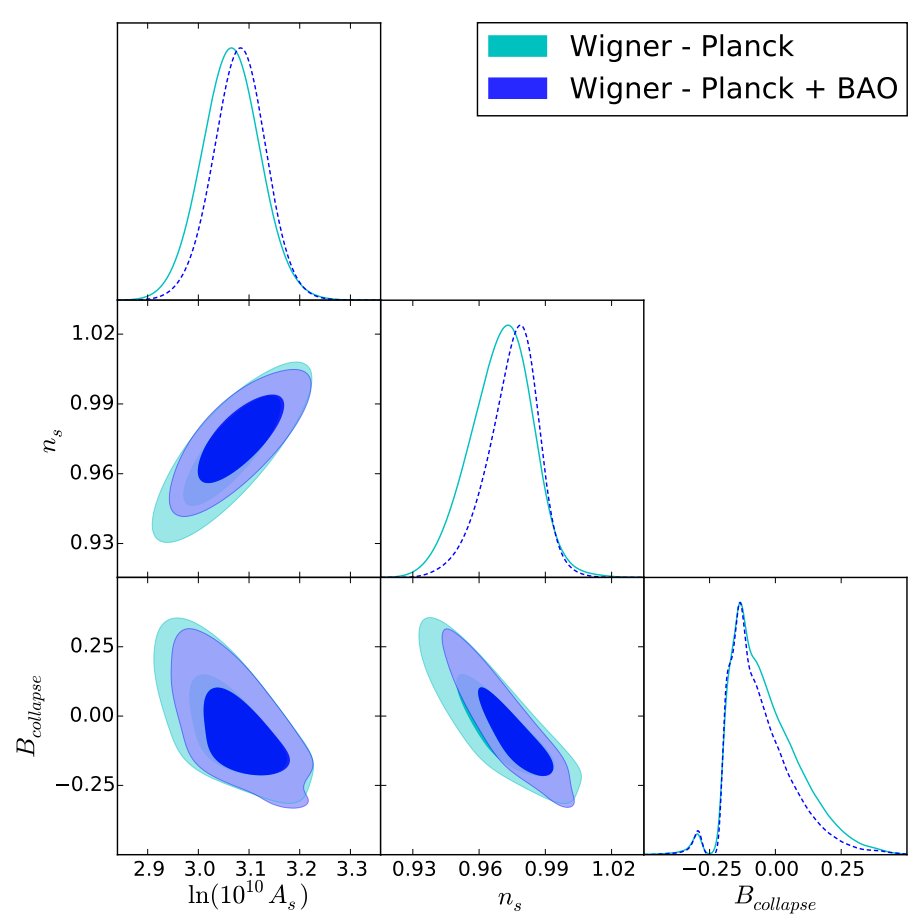

Figura 5.10. Distribuciones a posteriori marginalizadas para el modelo de Wigner obtenidas con los datos de Planck2015 y Planck+BAO. En la diagonal se muestran las distribuciones de densidad de probabilidad marginalizadas para un parámetro mientras que los otros paneles muestran los contornos de confianza a $1 \sigma$ y $2 \sigma$ de pares de los parámetros $\ln \left(10^{10} A_{s}\right), n_{s}$ y $B$.

\subsection{Modelo CSL}

El modelo CSL involucra modificar la ecuación de Schrödinger (ver sección 4.5). Lo interesante de esta propuesta es introducir el colapso espontáneo del campo inflatón como una consecuencia natural de la evolución del sistema y sin necesidad de introducir el colapso autoinducido de la función de onda del campo inflatón como una hipótesis adicional, para lo cual es imprescindible modificar las ecuaciones de la física que lo rigen.

El parámetro libre introducido en esta ocasión se parametriza del siguiente modo:

$$
\lambda_{k}=\lambda_{0}\left(\frac{1}{k}+\frac{\alpha}{k^{2}}\right)
$$

donde $\lambda_{0}=1 /|\tau|$ siendo $\tau$ es el tiempo conforme al inicio de inflación (que es negativo). El mismo depende del número de e-folds que dura inflación. Trabajos anteriores realizados por parte del grupo de investigación (Villalba (2016)) muestran que si bien el espectro primordial es afectado por el valor de $\lambda_{0}$ no lo es el espectro de anisotropías y polarización del FCR, por lo que no hay una preferencia por uno u otro valor en cuanto a contrastación con las observaciones se refiere, mientras éste sea congruente con las escalas de energía de inflación.

Para este caso se utiliza $\tau=1.5 \times 10^{8} \mathrm{Mpc}$ que corresponde a un potencial de inflación $V^{1 / 4}=10^{-4} M_{p l}$ y $N=68$ el número de $e$-folds. Si $\lambda_{k}=\frac{\lambda_{0}}{k}$ se recupera el modelo cosmológico estándar, por eso se contempla un apartamiento a segundo orden en $1 / k$ donde $\alpha$ es el parámetro libre a determinar.

Existe una restricción física muy importante sobre $\lambda_{k} \mathrm{y}$ es que su valor sea positivo. De 


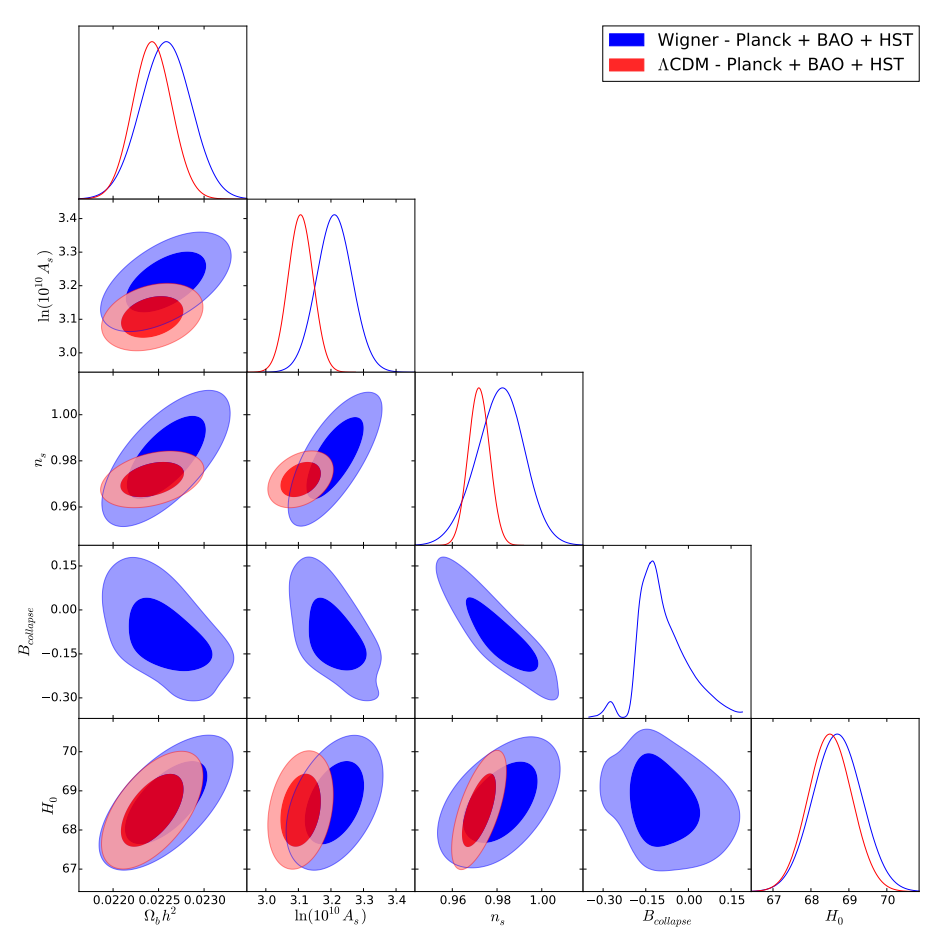

Figura 5.11. Distribuciones a posteriori marginalizadas para el modelo de Wigner obtenidas con los datos de Planck2015+BAO+HST. En la diagonal se muestran las distribuciones de densidad de probabilidad marginalizadas para un parámetro mientras que los otros paneles muestran los contornos de confianza a $1 \sigma$ y $2 \sigma$ de pares de los parámetros $\ln \left(10^{10} A_{s}\right)$, $n_{s}$ y $B$.

este modo y teniendo en cuenta que los valores relevantes de $k$ se encuentran entre $10^{-6} \mathrm{y}$ $10^{-1}$, resulta la siguiente restricción teórica:

$$
\alpha>-10^{-6}
$$

Por esta razón, se impone un límite inferior a los valores posibles del parámetro y su función de probabilidad a posteriori tiene una sola cola.

En la figura 5.12 se muestran los resultados para los parámetros cosmológicos de interés, resultando notable la coincidencia con los resultados obtenidos para el modelo fiducial. La predicción para $n_{s}$ no amplía notoriamente el intervalo de confianza, a diferencia de los modelos fenomenológicos. Los detalles cuantitativos pueden verse en la tabla 5.5. El valor de $\alpha$ resulta limitado por $4.3 \times 10^{-5}$ a un sigma (68\% de probabilidad).

Es importante destacar que la parametrización introducida para este modelo (ec. 5.10) afecta sólo a las escalas angulares más grandes (multipolos más pequeños) por lo que no se han incorporado los datos de $\mathrm{BAO}$ al análisis del mismo ya que estos son adecuados, por su naturaleza, para comparar resultados a escalas angulares más pequeñas (multipolos grandes).

Los indicadores estadísticos aportan los siguientes datos:

$$
\begin{aligned}
\Delta \chi^{2} & =-0.622 \\
\ln \mathcal{B}_{i j} & =-0.3
\end{aligned}
$$

resultando evidencia inconclusa en cuanto a su preferencia sobre el modelo estándar. 


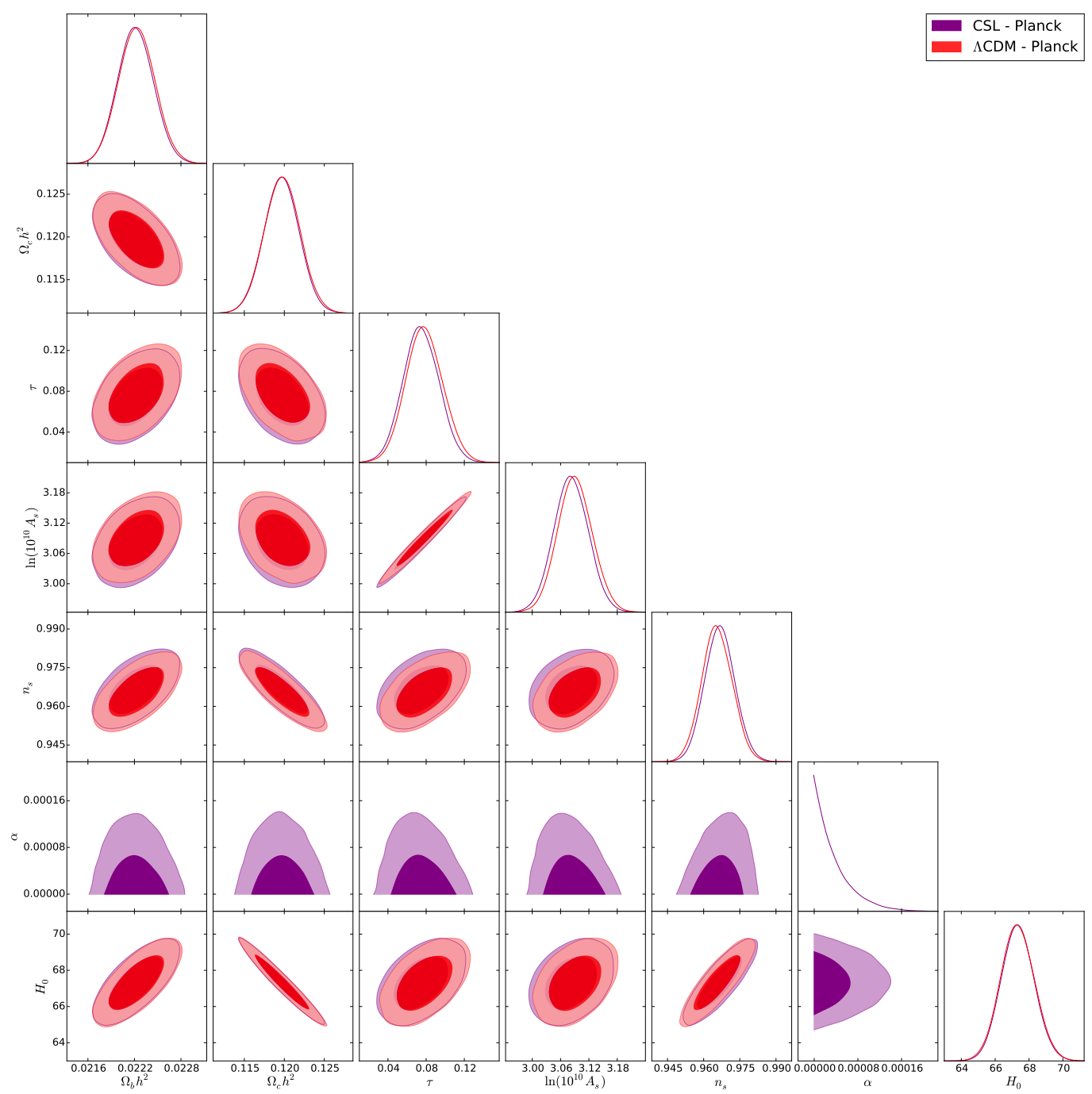

Figura 5.12. Distribuciones a posteriori marginalizadas para el modelo CSL obtenidas con los datos de Planck2015. En la diagonal se muestran las distribuciones de densidad de probabilidad marginalizadas para un parámetro mientras que los otros paneles muestran los contornos de confianza a $1 \sigma$ y $2 \sigma$ de pares de los parámetros cosmológicos.

\section{Determinación de los parámetros de slowroll y los potenciales de inflación}

Los parámetros de slowroll $\varepsilon_{1}$ y $\varepsilon_{2}$ pueden derivarse de las determinaciones de $A_{s}$ y $n_{s}$ :

$$
\varepsilon_{1}=\frac{V^{4}}{12 \pi^{2} m p^{4} A_{s}}, \quad \varepsilon_{2}=n_{s}-1+2 \varepsilon_{1},
$$

que se relacionan con las derivadas del potencial de inflación.

En la figura 5.14 se muestran los resultados obtenidos para $\alpha, \varepsilon_{1}$ y $\varepsilon_{2}$, en el caso del modelo CSL con los datos de Planck2015, junto con los resultados obtenidos para el modelo estándar. Se observan valores similares para $\varepsilon_{2}$ y valores más grandes para $\varepsilon_{1}$. La principal diferencia es que los contornos de Planck tienen un límite inferior con el que no contamos en el caso CSL. Esto es debido al tratamiento de la teoría semiclásica versus el abordaje de la cuantización mediante la variable de Mukhanov-Sasaki a través de la cual se construye el 


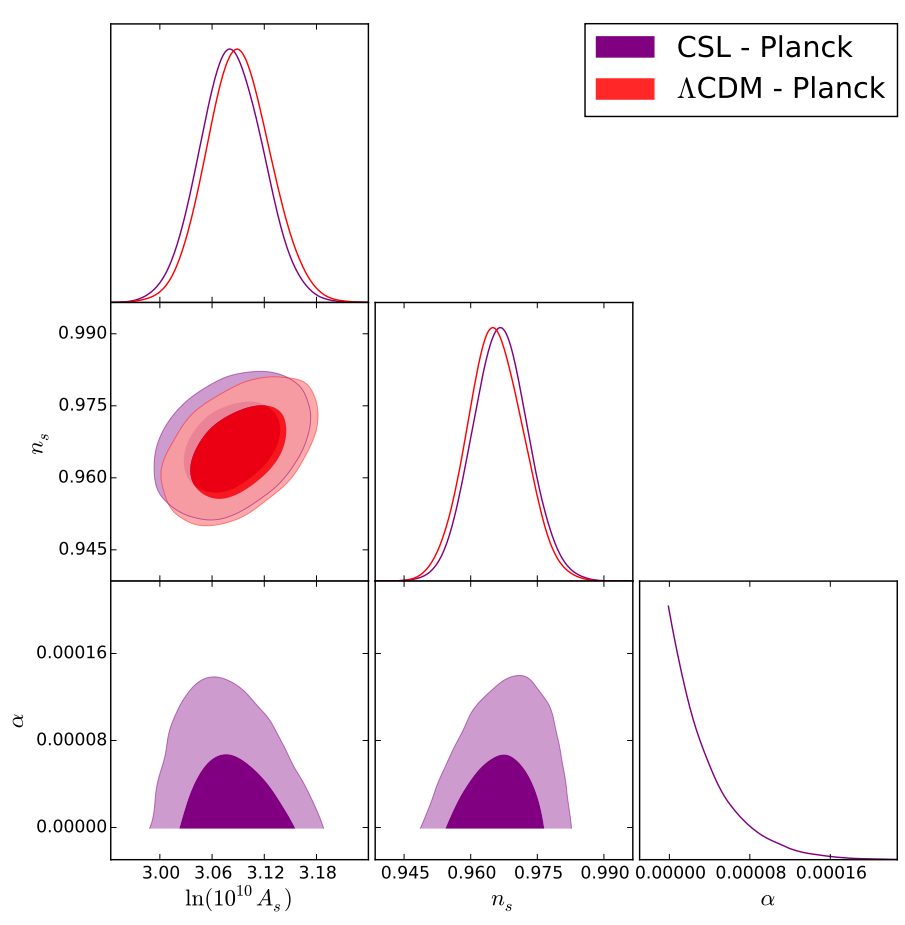

Figura 5.13. Distribuciones a posteriori marginalizadas para el modelo CSL obtenidas con los datos de Planck2015. En la diagonal se muestran las distribuciones de densidad de probabilidad marginalizadas para un parámetro mientras que los otros paneles muestran los contornos de confianza a $1 \sigma$ y $2 \sigma$ de pares de los parámetros $\ln \left(10^{10} A_{s}\right), n_{s}$ y $\alpha$.

\begin{tabular}{cr}
\hline Parámetro & Valor Medio $\pm 1 \sigma$ \\
\hline$\alpha$ & $<4.3 \times 10^{-5}$ \\
$n_{s}$ & $0.9667_{-0.0062}^{+0.0062}$ \\
$\ln \left(10^{10} A_{s}\right)$ & $3.0824_{-0.0363}^{+0.0367}$ \\
$\Omega_{b} h^{2}$ & $0.0222_{-0.0002}^{+0.0002}$ \\
$\Omega_{c} h^{2}$ & $0.1197_{-0.0022}^{+0.0022}$ \\
$100 \theta_{M C}$ & $1.0409_{-0.0005}^{+0.0005}$ \\
$\tau$ & $0.0747_{-0.0188}^{+0.0189}$ \\
$H_{0}$ & $67.3378_{-0.9721}^{+0.9674}$ \\
\hline
\end{tabular}

Tabla 5.5. Estimación de los parámetros cosmológicos para el modelo semiclásico CSL con los datos Planck2015.

modelo canónico. Para este caso existe una relación entre $r$ y $\varepsilon_{1}$ :

$$
r=\frac{P_{t}(k)}{P_{s}(k)} \propto \varepsilon_{1},
$$

y $r$ está restringido por los modos tensoriales. En el caso de los modelos semiclásicos, la amplitud de estos modos es despreciable y, por lo tanto, el límite superior para $r$ es aproximadamente $10^{-19}$. 


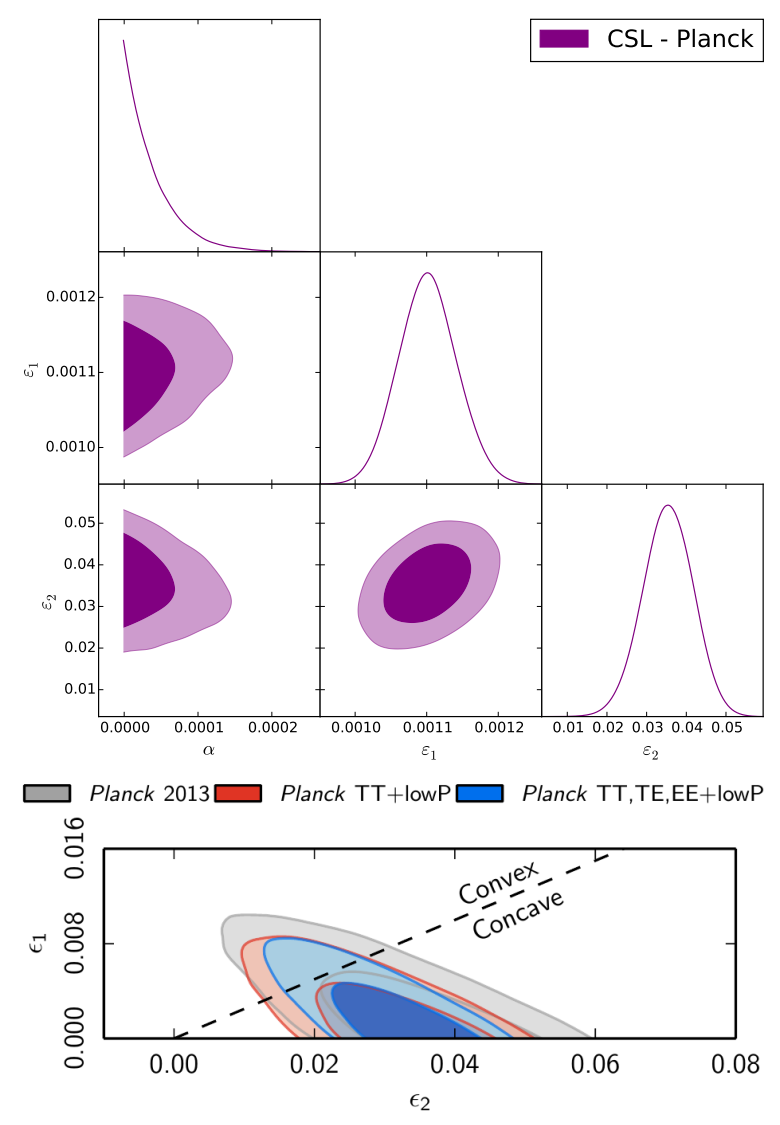

Figura 5.14. Arriba: distribuciones a posteriori marginalizadas para el modelo CSL obtenidas con los datos de Planck2015. En la diagonal se muestran las distribuciones de densidad de probabilidad marginalizadas para un parámetro mientras que los otros paneles muestran los contornos de confianza a $1 \sigma$ y $2 \sigma$ de pares del parámetro $\alpha$ y los parámetros derivados $\epsilon_{1}$ y $\epsilon_{2}$. Debajo: imagen extraída de la publicación Planck Collaboration et al. (2016a). El contorno que corresponde en comparación con los datos utilizados para esta tesis es el "Planck TT+lowP".

\subsection{Potenciales de inflación}

Se ha probado en trabajos previos (Landau et al., 2012) que la función likelihood cuenta con varios máximos si se busca estimar $A$ y $B$ simultáneamente en los esquemas de colapso mediante las cadenas de Markov-Monte Carlo.

Los primeros análisis estadísticos llevados para esta tesis de doctorado se llevaron a cabo con los datos Planck2013 y contemplaban al menos 10 valores distintos y arbitrarios de $A$ que se fijan a modo de estimar $B$ junto con el resto de los parámetros correspondientes al modelo cosmológico estándar. Sin embargo, el efecto neto que produce la incorporación de $A$ es una normalización que introduce una degeneración con el parámetro $A_{s}$. Simplemente fijando un valor determinado para $A$ que implique un valor aceptable de $A_{s}$, se puede proceder al análisis de un sólo caso y resulta representativo del modelo.

De la discusión de los modelos teóricos se sabe que $n_{s}$ está relacionado con los parámetros de slow-roll y éstos a su vez con el potencial de inflación. Entonces, si los esquemas de colapso permiten distintos intervalos de confianza para $n_{s}$ respecto al modelo estándar, será posible considerar distintas restricciones para los modelos de inflación, validando por ejemplo 
algunos que hayan sido descartados por el modelo cosmológico estándar y que se encuentren contemplados dentro de las restricciones para los esquemas de colapso.

Con este fin, se realizaron en colaboración con A. Pujol estimaciones para los valores de $n_{s}$ en el contexto del modelo de Wigner y los nuevos datos de Planck2015 y el esquema de Wigner. La figura 5.15 muestra la comparación entre los análisis previos y los actuales para $n_{s}$ a $1 \sigma$ junto con las cotas establecidas por los correspondientes datos de Plack.
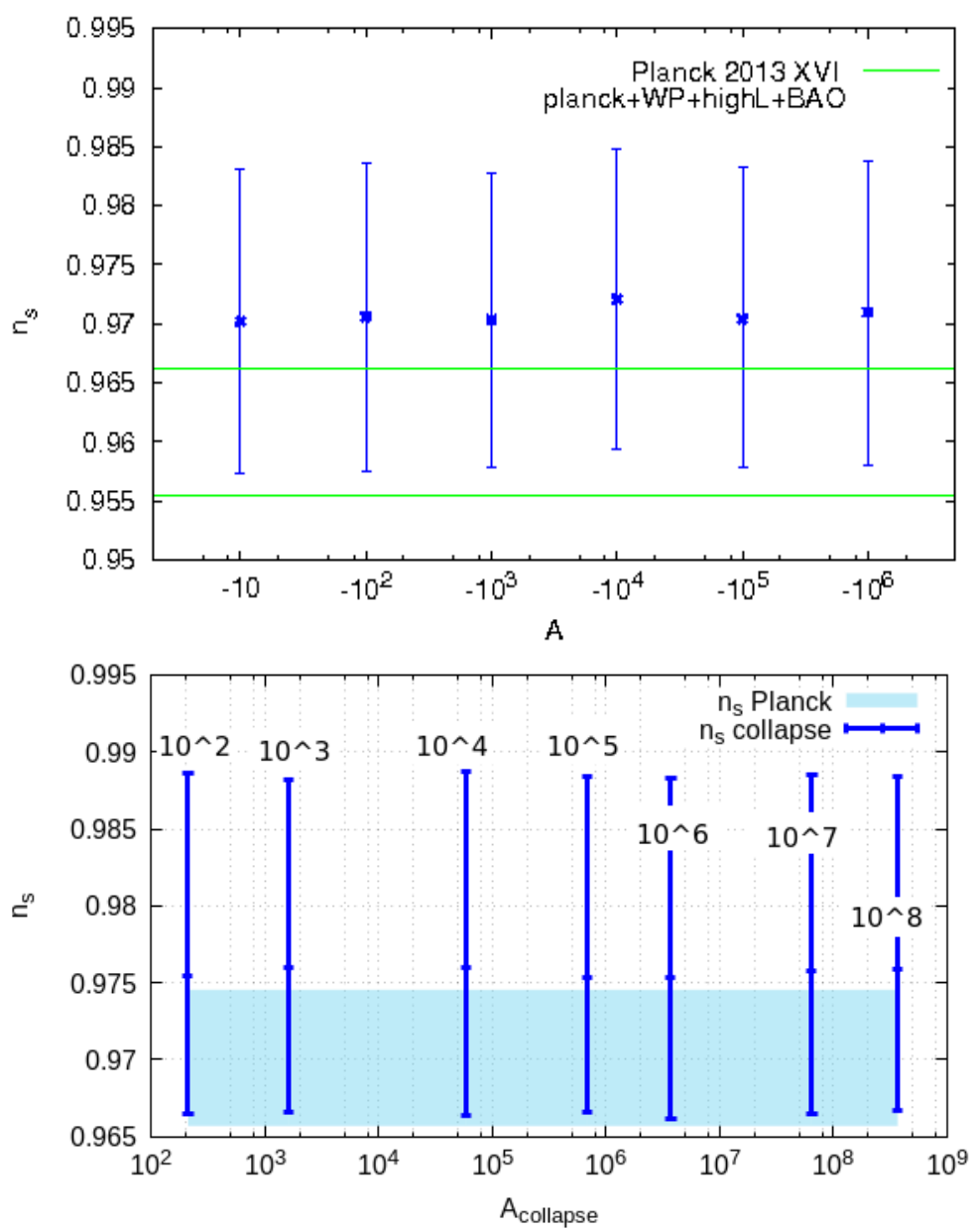

Figura 5.15. Arriba: estimaciones de $n_{s}$ y errores a $1 \sigma$ para distintas potencias de $A$ $\left(A=10^{i}, 1<i<9\right)$ con los datos de Planck2013. Debajo: estimaciones para $n_{s}$ y errores a $1 \sigma$ según distintos valores de $A$ tales que $A_{s}$ es similar al del modelo fiducial con datos de Planck2015. En ambos casos el análisis corresponde al esquema de Wigner en el caso en que el colapso de la función de onda del campo inflatón se produce dentro del horizonte. En todos los casos, el valor de $A$ es negativo.

La estimación para el intervalo de confianza es similar para todos los valores de $A$ (ver figura 5.16). En la figura 5.17 se muestran distintos contornos de confianza para dos valores determinados de $A$ en comparación con los resultados del modelo cosmológico estándar, para los datos Planck2015. El análisis para determinar qué modelos específicos de inflación son compatibles con los esquemas de colapso se encuentra en curso. 


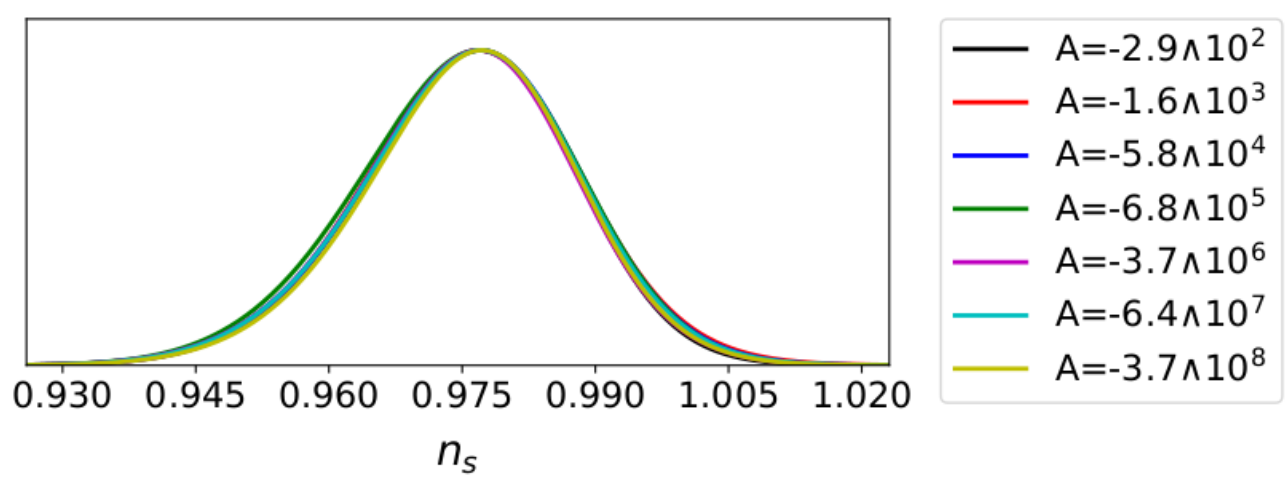

Figura 5.16. Probabilidad unidimensional a posteriori para $n_{s}$ según los distintos valores asignados al parámetro $A$. El modelo corresponde al esquema de Wigner en el caso que el colapso de la función de onda del campo inflatón ocurre dentro del horizonte. Se aprecia que ni el valor estimado para $n_{s}$, ni el intervalo de confianza dependen del valor de $A$. En todos los casos, el valor de $A$ es negativo.
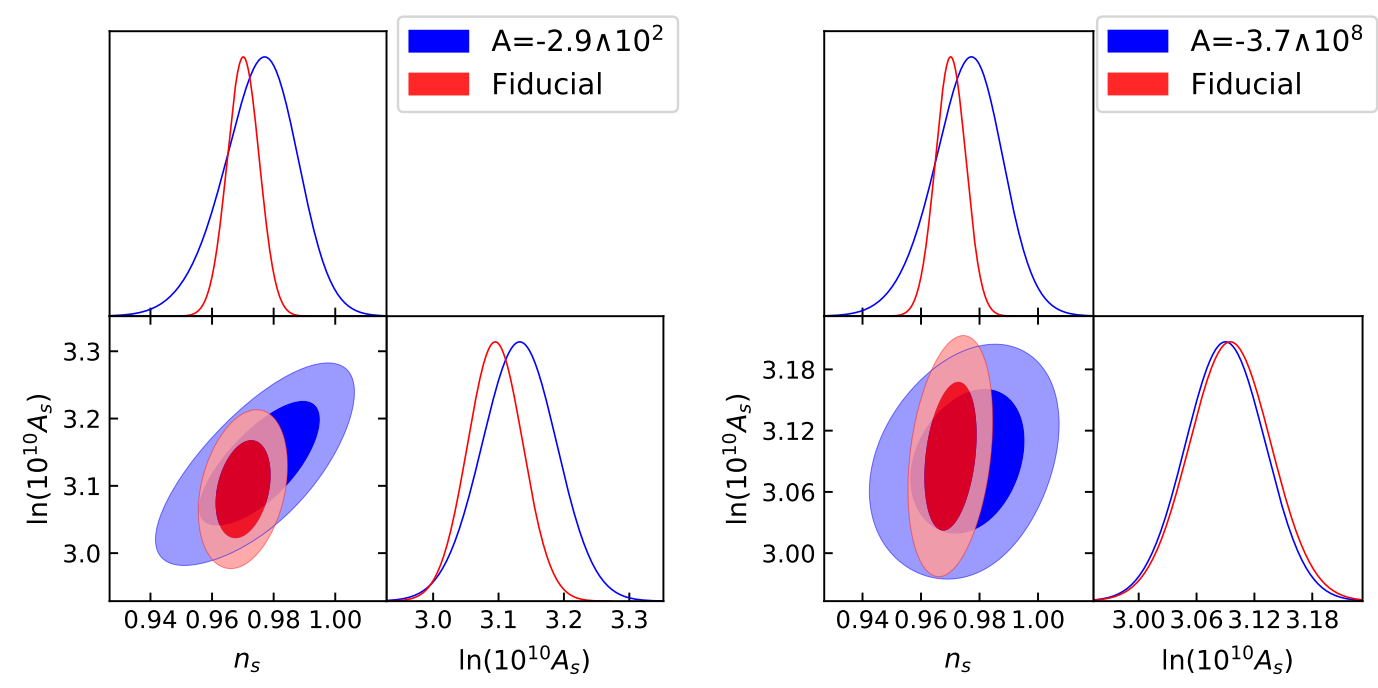

Figura 5.17. Distribuciones a posteriori marginalizadas para el modelo de Wigner obtenidas con los datos de Planck2015. En la diagonal se muestran las distribuciones de densidad de probabilidad marginalizadas para un parámetro mientras que los otros paneles muestran los contornos de confianza a $1 \sigma$ y $2 \sigma$ de pares de los parámetros $n_{s}$ y $A_{s}$ según distintos valores de $A$. 



\section{Capítulo 6}

\section{Conclusiones}

En esta tesis se consideró la hipótesis del colapso autoinducido de la función de onda de los modos del campo inflatón para explicar la formación de estructuras a partir de un estado inicial del Universo isótropo y homogéneo. Se consideraron dos alternativas para describir la dinámica del colapso: i) esquemas de colapso y ii) modelo CSL.

Se calculó el espectro primordial de fluctuaciones para modelos donde el colapso ocurre durante la época de inflación en los siguientes casos: a) esquemas de colapso Independiente, Newton y Wigner; b) modelo CSL.

A su vez, se modificó el código abierto CAMB que calcula las anisotropías en temperatura y polarización del FCR para incluir el espectro primordial de los modelos de colapso. Los resultados obtenidos indican que las predicciones para el esquema Independiente no se pueden distinguir de las respectivas del modelo cosmológico estándar. Por el contrario, para los esquemas de Newton, Wigner y el modelo CSL se encontraron predicciones distintas a las del modelo cosmológico estándar para varios valores de los parámetros libres de estos modelos. Por lo tanto, estos modelos son candidatos a ser comparados con los datos mediante análisis estadísticos para obtener límites sobre sus parámetros libres.

Se calculó el espectro primordial de fluctuaciones para modelos donde el colapso ocurre durante la época dominada por la radiación y la dinámica del mismo está dada por los esquemas de colapso. Se encontró que en varios casos particulares el modelo no puede explicar los datos observacionales. Para los casos que resultan viables, se encuentra que las predicciones de los modelos de colapso no se pueden distinguir de las respectivas para el modelo cosmológico estándar.

Se llevaron a cabo análisis estadísticos para comparar las predicciones de los modelos que incluyen la hipótesis del colapso de la función de onda de los modos del campo inflatón con datos recientes del Fondo Cósmico de Radiación, Oscilaciones Acústicas de Bariones y del Telescopio Espacial Hubble.

En todos los casos, los valores obtenidos para los parámetros cosmológicos del modelo estándar coinciden dentro de los $2 \sigma$ con los respectivos obtenidos para el modelo cosmológico estándar. A su vez se obtuvieron límites sobre los parámetros libres de los modelos de colapso. Por otra parte, los intervalos de confianza obtenidos sobre el parámetro $n_{s}$ son más amplios que los que se obtienen para el modelo cosmológico estándar. Dado que este parámetro está relacionado con el potencial de inflación, este resultado sugiere que potenciales que han sido descartados mediante análisis estadísticos realizados en el contexto del modelo estándar, no lo sean en el contexto de los modelos de colapso. 
Por otra parte, para determinar la preferencia de los datos entre los modelos de colapso y el modelo cosmológico estándar se calculó la evidencia bayesiana. En la mayoría de los casos los resultados indican preferencia moderada por el modelo cosmológico estándar. Hay dos excepciones: el modelo CSL, donde la evidencia bayesiana indica preferencia inconclusa por ambos modelos y el esquema Wigner con datos de Planck2015 y BAO donde la evidencia bayesiana indica preferencia moderada por el modelo de colapso.

La tabla 6.1 resume los modelos de colapso que fueron contrastados con datos observacionales recientes de interés cosmológico y señala en color la combinación que es favorecida moderadamente por la evidencia bayesiana frente al modelo cosmológico estándar.

\begin{tabular}{l|l|l|l}
\hline Modelo & $\mathbf{P 1 5}$ & $\mathbf{P 1 5 + B A O}$ & $\mathbf{P 1 5 + B A O + H S T}$ \\
\hline Newton & $\begin{array}{l}\text { prob. a posteriori de } \\
\text { B no gaussiana }\end{array}$ & $\begin{array}{l}\text { no incorpora } \\
\text { nuevas restricciones }\end{array}$ & no se realiza \\
\hline Wigner & $\begin{array}{l}\text { mejor evidencia ba- } \\
\text { yesiana que Newton }\end{array}$ & $\begin{array}{l}\text { preferencia modera- } \\
\text { da frente al modelo } \\
\text { estándar }\end{array}$ & $\begin{array}{l}\text { no resuelve la tensión } \\
\text { entre datos }\end{array}$ \\
\hline CSL & $\begin{array}{l}\text { se encuentra } \\
\text { una cota para } \alpha\end{array}$ & no se realiza & no se realiza \\
\hline
\end{tabular}

Tabla 6.1. Resultados del análisis estadístico de modelos de colapso con datos observacionales recientes de interés cosmológico.

En resumen, a partir de los análisis estadísticos se puede afirmar que las predicciones de los modelos de colapso permiten explicar los datos actuales del FCR, BAO y HST, dando así una explicación teórica al surgimiento de las inhomogeneidades y anisotropías del Universo primordial. 


\section{Apéndice A}

\section{Acrónimos}

BAO Barionic Acoustic Oscillations

CAMB Code for Anisotropies in the Microwave Background

CMB Cosmic Microwave Background

COBE Cosmic Microwave Background Explorer

CSL Continuous Spontaneous Localization

HST Hubble Space Telescope

FCR Fondo Cósmico de Radiación

WMAP Wilkinson Microwave Anisotropy Probe 



\section{Bibliografía}

Anderson, L. et al. 2014, Mon. Not. Roy. Astron. Soc., 441, 24

Baumann, D. 2009, ArXiv e-prints, 0907.5424

Benetti, M., Landau, S. J., \& Alcaniz, J. S. 2016, JCAP, 12, 035

Bengochea, G. R. \& León, G. 2017, Physics Letters B, 774, 338

Bennett, C. L., Larson, D., Weiland, J. L., et al. 2013, ApJS, 208, 20

Beutler, F., Blake, C., Colless, M., et al. 2011, Mon. Not. Roy. Astron. Soc., 416, 3017

BICEP2/Keck Collaboration, Planck Collaboration, Ade, P. A. R., et al. 2015, Physical Review Letters, 114, 101301

Cañate, P., Pearle, P., \& Sudarsky, D. 2013, Physical Review D, 87, 104024

Das, S., Louis, T., Nolta, M. R., et al. 2014, JCAP, 4, 014

de Unánue, A. \& Sudarsky, D. 2008, Physical Review D, 78, 043510

Diez-Tejedor, A., Leon, G., \& Sudarsky, D. 2012, Gen.Rel.Grav., 44, 2965

Diez-Tejedor, A. \& Sudarsky, D. 2012, JCAP, 7, 45

Eisenstein, D. J., Zehavi, I., Hogg, D. W., et al. 2005, ApJ, 633, 560

Eisentein, D. J. \& Bennett, C. L. 2008, Cosmic sound waves rule, Feature Article, Physics Today

Feroz, F. \& Hobson, M. P. 2008, Mon. Not. Roy. Astron. Soc., 384, 449

Feroz, F., Hobson, M. P., \& Bridges, M. 2009, Mon. Not. Roy. Astron. Soc., 398, 1601

Feroz, F., Hobson, M. P., Cameron, E., \& Pettitt, A. N. 2013, arXiv e-prints, 1306.2144

Gil-Marín, H. et al. 2016, Mon. Not. Roy. Astron. Soc., 460, 4210

Grishchuk, L. \& Sidorov, Y. 1990, Phys. Rev., D 42, 3413

Hooft, G., Giddings, S. B., Rovelli, C., et al. 2016, ArXiv e-prints, 1609.01725

Jeffreys, H. 1961, 3rd edn. OUP

Kiefer, C. \& Joos, E. 1999, in Lecture Notes in Physics, Berlin Springer Verlag, Vol. 517, Quantum Future: From Volta and Como to the Present and Beyond, ed. P. Blanchard \& A. Jadczyk, 105 
Kiefer, C. \& Polarski, D. 2009, Adv. Sci. Lett., 2, 164

Kinney, W. H. 2005, Phys.Rev., D72, 023515

Landau, S., León, G., \& Sudarsky, D. 2013, Physical Review D, 88, 023526

Landau, S. J., Scóccola, C. G., \& Sudarsky, D. 2012, Physical Review D, 85, 123001

León, G., Bengochea, G. R., \& Landau, S. J. 2016, European Physical Journal C, 76, 407

León, G., De Unánue, A., \& Sudarsky, D. 2011, Classical and Quantum Gravity, 28, 155010

León, G., Kraiselburd, L., \& Landau, S. J. 2015a, Physical Review D, 92, 083516

León, G., Landau, S. J., \& Piccirilli, M. P. 2014, Physical Review D, 90, 083525

León, G., Landau, S. J., \& Piccirilli, M. P. 2015b, European Physical Journal C, 75, 393

León, G. \& Sudarsky, D. 2010, Classical and Quantum Gravity, 27, 225017

Lewis, A. \& Bridle, S. 2002, Phys. Rev., D66, 103511

Lewis, A., Challinor, A., \& Lasenby, A. 2000, Astrophys. J., 538, 473

León, G., Majhi, A., Okon, E., \& Sudarsky, D. 2017a, ArXiv e-prints, 1712.02435

León, G., Majhi, A., Okon, E., \& Sudarsky, D. 2017b, Phys. Rev., D96, 101301

Ma, C.-P. \& Bertschinger, E. 1995, Astrophys. J., 455, 7

Perez, A., Sahlmann, H., \& Sudarsky, D. 2006, Classical and Quantum Gravity, 23, 2317

Piccirilli, M. P., León, G., Landau, S. J., Benetti, M., \& Sudarsky, D. 2018, International Journal of Mothern Physics D, 28, 1950041

Planck Collaboration, Ade, P. A. R., Aghanim, N., et al. 2014a, A\&A, 571, A15

Planck Collaboration, Ade, P. A. R., Aghanim, N., et al. 2014b, A\&A, 571, A16

Planck Collaboration, Ade, P. A. R., Aghanim, N., et al. 2016a, A\&A, 594, A20

Planck Collaboration, Ade, P. A. R., Aghanim, N., et al. 2016b, A\&A, 594, A13

Planck Collaboration, Aghanim, N., Arnaud, M., et al. 2016c, A\&A, 594, A11

Planck Collaboration, Aghanim, N., Ashdown, M., et al. 2017, A\&A, 599, A51

Pujol, A. 2018, Restricciones observacionales a potenciales inflacionarios en el contexto del colapso cuántico, Tesis de Licenciatura, UBA

Reichardt, C. L., Shaw, L., Zahn, O., et al. 2012, ApJ, 755, 70

Riess, A. G., Macri, L. M., Hoffmann, S. L., et al. 2016, ApJ, 826, 56

Ross, A. J., Samushia, L., Howlett, C., et al. 2015, Mon.Not.Roy.Astron.Soc., 449, 835

Rovelli, C. 2003, International Journal of Modern Physics D, 12, 1509

Ryden, B. 2006, Introduction to Cosmology 
Scóccola, C. 2009, PhD thesis, Facultad de Ciencias Astronómicas y Geofísicas, Universidad Nacional de La Plata

Seljak, U. \& Zaldarriaga, M. 1996, Astrophys. J., 469, 437

Sudarsky, D. 2011, International Journal of Modern Physics D, 20, 509

Trotta, R. 2007, Mon. Not. Roy. Astron. Soc., 378, 72

Villalba, M. 2016, Estudio del modelo inflacionario de colapso con mecanismo CSL utilizando datos del FCR, Tesis de Licenciatura, UBA

Zaldarriaga, M. \& Seljak, U. 1997, Physical Review D, 55, 1830 